\title{
Distributed Leadership in Schools
}

\author{
Andrew Leonard Hill
}

BSc DipEd. BEd MACE

March 2008

A thesis submitted in total fulfilment of the requirements for the degree of Doctor of Philosophy, Faculty of Education.

The University of Melbourne. 


\begin{abstract}
This study examines the concept of distributed leadership as applied to schools. Various interpretations of the term, 'distributed leadership' are described in the literature: Distributed leadership is presented by some writers as another version of team leadership, shared leadership, democratic leadership and the like. Other writers describe distributed leadership as normative practice - as the way leadership ought to be enacted. The term, 'distributed leadership', as used in this thesis, refers to a dualistic, two-dimensional explanation of organisational life. Here, a naturalistic, causal-computational view of organisational action stands alongside a phenomenological, qualitative view of the essence of leadership. Whist the former may offer a universal, third-person view of organisational functioning; the viewpoint presented by the latter offers distinctive, first-person experiences of leadership.
\end{abstract}

Although correctly emphasising the role of environment and culture in leadership as distributed, it is argued here that distributed leadership shares the theoretical and epistemological problems of all leadership theories and lacks explanatory power. This conclusion is reached by means of a naturalistic coherentist theory of knowledge, which recognises the theory-dependence of empirical data. Coherence is adjudicated according to the superempirical virtues of conservatism, simplicity, consistency, comprehensiveness, fecundity, explanatory unity, refutability, and learnability. Naturalistic coherentist epistemology evaluates theories according to their coherence relations with other trusted theories, such as the theories of natural science.

As part of the methodology, interview data were collected via interviews with four designated leaders in a single Victorian Government primary school. In keeping with a naturalistic coherentist theory of knowledge, the interview data are not given foundational status as evidence. Since, a coherentist epistemology requires analysis of the theories at the core of any research investigation; a wide-ranging theoretical 
analysis is also an important part of the methodology. Narrative texts are also analysed in accordance with the hermeneutic circle.

Hermeneutic analysis of leadership narratives reveals the phenomenological character of leadership in folk psychology. Theoretical analysis of formal leadership theories such as trait theories, situation theories, contingency theories and transformational leadership reveals that leadership is not amenable to global generalisations in the guise of formal, rule-governed explanations. Yet, heuristic analysis suggests that cognitive theory, and therefore theories of mind, are centrally important to the explanation of organisational action and leadership.

Organisational functioning may be described according to the causalcomputational terms of distributed cognition theory. This theory offers a coherent, explanation of human learning in terms of the physical interaction of mind, body and world. In contrast, the phenomenological perspective of folk psychology portrays the essence of leadership through the qualitative differences of human experience. Distributed leadership theory, as it is described here, places these two perspectives alongside each other within a single two-dimensional account of organisational life.

As a concept of phenomenology, leadership is not amenable to generalisation as a context-free concept. When understood naturalistically as an emergent property of how individuals learn, however, distributed leadership becomes synonymous with human cognition. Therefore, school effectiveness and improvement are more appropriately understood as matters of learning rather than leadership. Distributed leadership in schools, as presented in this thesis seeks to provide a theoretical framework for the analysis of school functioning. This framework enables school functioning to be explained in general terms whilst also acknowledging the firstperson experience of organisational life, as perceived by its participants. 


\section{Declaration}

This is to certify that

(i) This thesis comprises only my original work towards the $\mathrm{PhD}$ except where indicated in the Preface,

(ii) Due acknowledgement has been made in the text to all other material used,

(iii) The thesis is less than 100,000 words in length, exclusive of tables, maps, bibliographies and appendices.

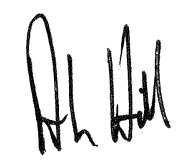

Andrew Leonard Hill

Melbourne, March 2008. 


\section{Preface}

A paper titled Narratives of Leadership, which was derived from a draft version of Chapter 4 of this thesis, was presented at Staying on Track in November 2005, being the Postgraduate Research Conference of the Education Faculty, University of Melbourne. A paper derived from a draft version of Chapter 2 of this thesis was presented at the Staying on Track conference in November 2006 and published as Hill (2007).

This thesis draws on work carried out prior to $\mathrm{PhD}$ candidature enrolment, being case study, interview research conducted during candidature of Master of Education by research. The findings of that research were not previously published in a thesis, paper or other publication due to the cessation of MEd candidature, for the purpose of immediately commencing $\mathrm{PhD}$ candidature.

The research methodology utilised during MEd candidature consisted of eight interviews, conducted in accordance with Human Ethics requirements, in two Victorian Government Primary Schools, and the subsequent preparation of interview transcripts. This method of investigation took place under the umbrella of the Cooperative Research Project of the Victorian Education Department, Victorian Association of State Secondary Principals, Victorian Primary Principals Association, and The University of Melbourne (see Cooperative Research Project, 1998). 


\section{Acknowledgements}

I dedicate this thesis to the cherished memory of my grandfather and respected educator, Leonard Cooke, who sowed the seeds of this work in me; and to the memory of my dearly-loved father, Norman Hill, who engendered the perseverance needed to finish it.

I would also like to thank Gabriele Lakomski and Brian Caldwell for their valued oversight and advice. In particular, Gabriele provided valuable advice during my $\mathrm{PhD}$ candidature and enabled me to keep in touch with the research of my fellow doctoral students through her regular Doctoral Research Seminars. 


\section{Table of Contents}

Chapter 1: $\quad$ Introduction.................................................................... 1

1.1 The glorious leader 1

1.2 What is leadership? 4

1.3 Language, culture and thought 5

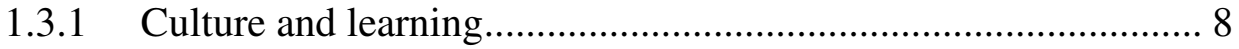

1.3.2 The interdependence of fact and value ................................... 10

1.4 The commonsense notion of leadership 12

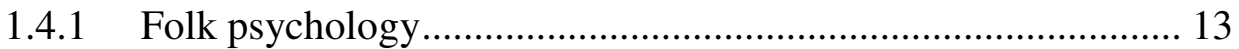

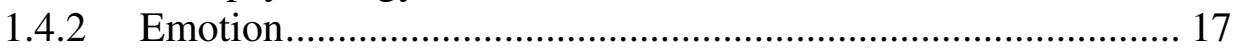

1.4.3 Informal versus formal theories ............................................. 19

1.5 Self-reference in formal theories of leadership 21

1.5.1 The logical paradox of self-reference ..................................... 21

1.5.2 Inconsistency or incompleteness? ......................................... 23

1.6 Leadership and distributed social action 24

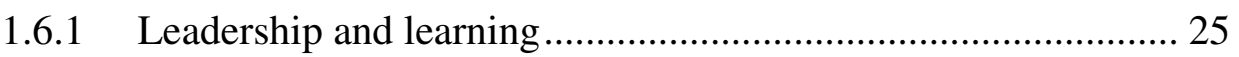

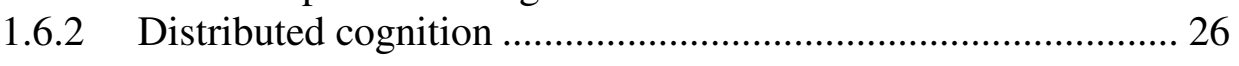

$\begin{array}{lll}1.7 & \text { Levels of analysis } & 27\end{array}$

$\begin{array}{lll}1.8 & \text { Naturalistic coherentism } & 30\end{array}$

1.8.1 Methodological considerations ................................................. 33

1.9 Distributed leadership $\quad 35$

1.10 Synopsis of the following chapters 38

Chapter 2: $\quad$ Epistemological considerations .......................................40

$\begin{array}{lll}2.1 & \text { Introduction } & 40\end{array}$

2.2 Distributed leadership theories 44

2.3 The epistemological claims of this thesis 49

2.4 Naturalistic Coherentism 51

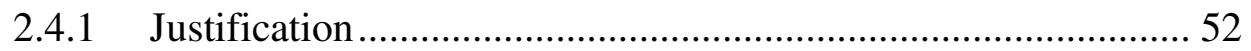

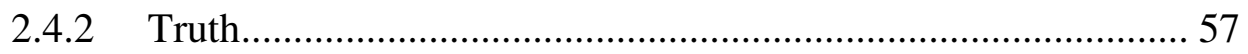


2.4.3 Belief. 60

2.5 Limitations to knowledge 65

2.5.1 The limitations of naturalistic coherentism ............................. 66

2.5.2 The limitations of empirical evidence ...................................... 68

2.5.3 The limitations of theories .................................................... 72

2.6 Creating better theories 73

2.6.1 The construction and evaluation of theories ............................. 75

2.7 The place of experience and explanation 78

Chapter 3: $\quad$ Methodology …...............................................................83

3.1 Introduction $\quad 83$

3.2 The theory-dependence of research methods 86

3.3 Methodology explained $\quad 88$

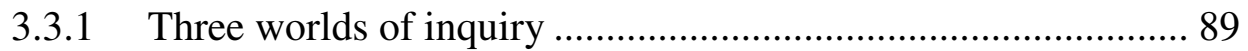

3.3.2 Heuristic methods ................................................................. 91

3.3.3 Hermeneutics .................................................................. 95

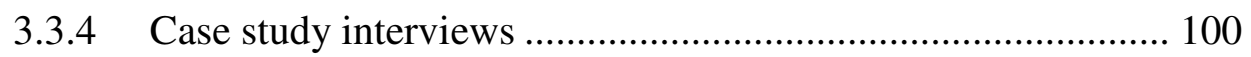

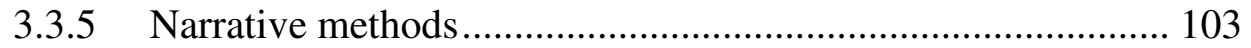

3.3.6 Theoretical methods.................................................................. 106

3.3.7 Triangulation.......................................................................... 107

3.4 The research methods of this study 108

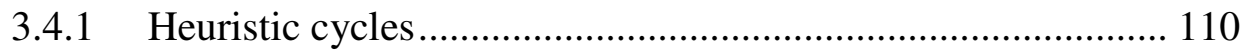

3.4.2 Hermeneutic analysis .......................................................... 112

3.4.3 Structured interviews .......................................................... 113

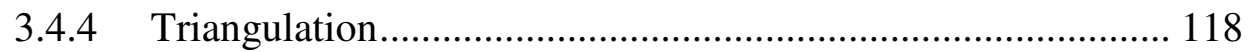

Chapter 4: $\quad$ Leadership narratives ......................................................120

$\begin{array}{lll}4.1 & \text { Introduction } & 120\end{array}$

$\begin{array}{lll}4.2 & \text { Schemas of leadership } & 121\end{array}$

4.2.1 The schema of leadership as competence ............................... 123

4.2.2 The schema of leadership as influence ................................... 129

4.2.3 The schema of political-leadership .......................................... 134

4.2.4 The schema of leadership-as-image ..................................... 138

4.2.5 The schema of leadership-as-social-relations .......................... 141

4.2.6 The schema of leadership-as-performance .............................. 144

4.2.7 The schema of leadership-as-innovation ............................... 147

4.3 An absence of leadership schemas 149

$\begin{array}{lll}4.4 \text { Conclusion } & 151\end{array}$ 
Chapter 5: $\quad$ Narrative meaning............................................................152

$\begin{array}{lll}5.1 & \text { Introduction } & 152\end{array}$

5.2 From situational narrative to grand narrative 153

$\begin{array}{lll}5.3 & \text { Narrative knowing } & 156\end{array}$

5.4 Schemas as organisers of knowledge 161

$\begin{array}{lll}5.5 & \text { Narrative structure } & 163\end{array}$

$\begin{array}{lll}5.6 & \text { Propositional attitudes } & 169\end{array}$

5.6.1 The distinction between theoretical propositions and propositional attitudes........................................................... 172

5.6.2 The intensional content of propositional attitudes.................. 173

5.6.3 The ambiguity of propositional attitudes ................................ 176

5.7 Conclusion 182

\section{Chapter 6: $\quad$ Leadership as information processing..................... 183}

6.1 Introduction 183

6.1.1 The lack of predictive power in formal leadership theories .... 184

6.1.2 Cause, effect and explanation ............................................. 187

6.1.3 Phenomenal experience in narrative ..................................... 192

6.1.4 The computational character of sentences .............................. 194

6.2 Cognition as information processing 200

6.2.1 Schemas as aids to information processing ............................ 201

6.2.2 The physical and phenomenological realisation of Information

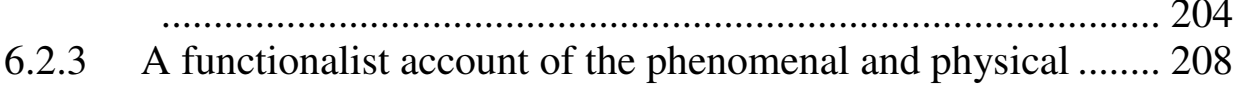

6.2.4 The global nature of information ............................................ 210

6.2.5 Consciousness and free-will .................................................. 211

$\begin{array}{lll}6.3 \text { Conclusion } & 214\end{array}$

Chapter 7: Distributed cognition .........................................................216

$\begin{array}{lll}7.1 & \text { Introduction } & 216\end{array}$

$\begin{array}{lll}7.2 \text { Mind } & 217\end{array}$

7.2.1 The routine tactical moves of leaders ..................................... 218

7.2.2 Leadership and the meaning of phenomenal experience ......... 221

7.2.3 Can concerted group action constitute leadership?................. 222

7.2.4 Folk psychology............................................................... 225

7.2.5 A computational model of mind ........................................ 229 


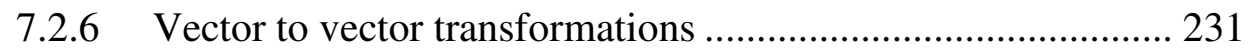

7.2.7 The limitations of computational systems ............................. 235

$\begin{array}{lll}7.3 & \text { Body } & 237\end{array}$

7.3.1 The Somatic Marker Hypothesis ............................................. 239

7.3.2 Emotion and value ........................................................... 243

$\begin{array}{lll}7.4 \text { World } & 245\end{array}$

7.4.2 A neural model of narrative structure ..................................... 250

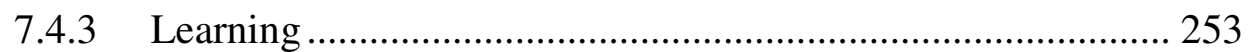

7.4.4 The impracticality of a central planner ................................. 255

7.4.5 Levels of analysis......................................................... 258

$\begin{array}{lll}7.5 & \text { The distributed mind } & 261\end{array}$

Chapter 8: $\quad$ Distributed Leadership ..........................................263

8.1 Introduction 263

8.2 Leadership in organisations 269

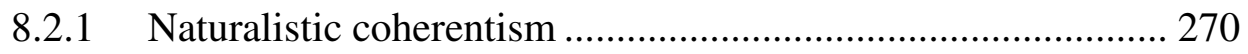

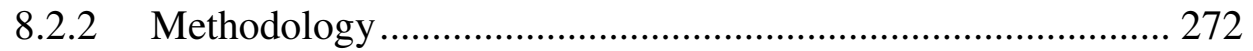

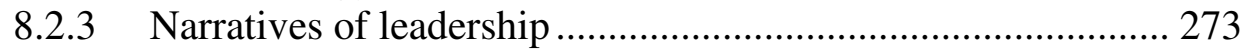

8.2.4 Narrative meaning.................................................................. 278

8.2.5 Leadership in information space .......................................... 282

8.2.6 Distributed cognition ............................................................ 285

8.3 Distributed Leadership 287

8.3.1 Distributed leadership: prescription or description................. 290

8.3.2 Cognition, culture and place ..................................................... 291

8.3.3 Cognition, leadership and language ...................................... 294

8.3.4 The distribution of leadership .............................................. 296

$\begin{array}{lll}8.4 \text { Conclusion } & 299\end{array}$

8.4.1 To what extent are organisations the outworking of human cognition as described by theories of distributed cognition?.. 299

8.4.2 To what extent can the functioning of schools as educational organisations be explained in terms of leadership?

8.4.3 Given the answers to the previous questions, what can a theory of distributed leadership offer?

References

Appendix 1: Interview B5 Transcript .....................................................343

Appendix 2: Interview B6 Transcript ........................................................355 
Appendix 3: Interview B7 Transcript .......................................................362

Appendix 4: Interview B8 Transcript ........................................................372

Appendix 5: Analysis of an interview with a leading teacher (Interview B8)

Analysis 


\section{List of Tables}

Table 3.1

Table 5.1

Table 5.2

Table 5.3

Table 7.1

Table 7.2
Interview questions 117

Alignment of narrative purpose with leadership schemas ..... 155

Tensions of situational and grand narrative. 159

The correspondence of tropes of and modes of emplotment.. 165

Input, output and target data for the XOR problem 232

Distributed cognition: levels of analysis 258

\section{List of Figures}

Figure 1.1

Figure 1.2

Figure 1.3

Figure 1.4

Figure 3.1

Figure 5.1

Figure 6.1

Figure 7.1

Figure 7.2

Figure 7.3

Figure 7.4

Figure 7.5

Figure 7.6
Theories of folk-psychology: a taxonomy..... 16

Recursive growth in knowledge 31

Three levels of analysis for distributed cognition.................... 33

Emergent and supervenient relations ...................................... 34

Three worlds of inquiry 90

Three dimensions of narrative 157

Continuum of explicit and implicit propositional attitudes .... 191

Parallel distributed network design: XOR Problem 232

XOR surface plot (after training) 233

XOR surface plot (in the early stages of training)

A Necker Cube 247

Parallel distributed processing - Levels of analysis 260

Random and Scale-free network configurations 261 


\title{
Chapter 1: Introduction
}

\begin{abstract}
Can questions of school organisation and improvement be justifiably reduced to problems of school leadership? That is the central question addressed in this thesis. Organisations may be explained naturalistically according to distributed cognition theory. However, because leadership qualities are more appropriately described as categories of phenomenology, they are not compatible with a naturalistic, causal-computational account. Nevertheless, the limits of theoretical accounts leave room for leadership, as grasped phenomenologically, to be accepted as an, as yet, unexplained product of human free-will and intentionality. Hence, distributed leadership is proposed here as a special case of distributed agency, a theory that informally admits human intentionality into a formal description of distributed cognition.
\end{abstract}

\subsection{The glorious leader}

Devotion,

by Thomas Campion, 1567-1619.

Follow thy fair sun, unhappy shadow!

Though thou be black as night,

And she made all of light,

Yet follow thy fair sun, unhappy shadow!

Follow her, while yet her glory shineth!

There comes a luckless night

That will dim all her light;

And this the black unhappy shade divineth.

Follow still, since so thy fates ordained!

The sun must have his shade,

Till both at once do fade, -

The sun still proud, the shadow still disdained.

(Campion, 1900)

Here is the leader, displayed in radiant glory, the one who gives identity and purpose to the follower. Inglorious, the follower's melancholy emanates from a 
dependent and subservient relationship to the leader. One cannot exist without the other, yet the leader has honour over the follower. The image of leadership evoked by this poem may be associated with the traditional authority of kings and emperors in early times. This view of leadership persists in many ways in $21^{\text {st }}$ Century thought, but is at odds with views that emphasise the interdependence and mutual honour of people in communities and organisations. From his research, Argyris (1961, p. 327) reports that 'reality for the subjects being studied in an organisational setting [is] a pattern of variables (leadership, managerial controls, and so on) so inextricably interrelated that it [is] impossible for the subjects to separate them and still speak of reality'. The qualities associated with leadership appear to fade away when the physical day-to-day realities of organisational life are described.

In contrast with traditional views that emphasise the agency of leaders over others, recent developments in cognitive theory recognise the distribution of thought and action in matters of social and organisational action. If, as this line of thinking suggests, organisations do function according to a network of interdependent relations, then answers to problems of organisational functioning will not be found in theories that emphasise the traits and qualities of individuals.

Matters of school organisation are often addressed as leadership problems, in whole or in part. Such an approach is evident, for example, in the Blueprint Initiative of the Victorian Department of Education, Accelerated Development for High Potential Leaders (Expression of Interest for Master in School Leadership, and if applicable, Young Leaders Program, through the Blueprint Initiative: Accelerated Development for High Potential Leaders, 2004). This initiative adopts a transformational leadership model and aims to develop 'the leadership capacity' of 'leadership aspirants' who have been identified as 'high potential leaders'. This assumes that some people possess an extraordinary, latent leadership potential, so that they have a disposition to become leaders that others do not and are able to expand their leadership capabilities through training. It is assumed that people who have these qualities can be distinguished from those who do not, and that such people will most likely, in leadership terms, provide a good return for the investment of valuable resources. Policy makers tend to emphasise the agency of those occupying 
formal leadership positions - over and above the agency of others in schools - and this is one such example.

Leadership is often coined in a matter-of-fact way. It is as if we all know what leadership is, so that the problem is really about how we set about achieving it. There is also a pervasive assumption that school improvement problems are leadership problems. A theory of distributed leadership is introduced in this thesis as a special case of distributed agency. Distributed leadership theory offers an alternative to leader-centric leadership theories. It has at its core a theory of distributed cognition; but also acknowledges the prevalence of assumptions of intentionality and free-will in the day-to-day sense-making of principals and teachers in schools.

My thesis is that school organisation problems are better understood as learning problems, that is, as problems of human cognition. As an alternative to a focus on leaders or leadership processes, attention should be given to the detailed particulars, or fine-grained features, of organisations. The answers to school organisation problems lie in the detailed study of the causal relations between people, artefacts and environment, not in a general theory of leadership. Whilst this view clearly downplays the causal influence of individuals labelled as leaders, it is important to recognise that all individuals, including formal leaders, make important contributions to the health and vitality of organisations. Nevertheless, their contributions depend significantly upon the characteristics of the situations in which they work; that is, the patterns that arise from the interdependent relations of people, artefacts, information and environment. This is not to say that those who hold formal leadership positions in schools should not seek ways to improve what they do, nor does it belittle the importance of their cultural and functional roles. Rather it is to say that schools undergo change through the adaptive interaction of many different elements of knowledge, culture, and situation. As principals and other school leaders gain a greater understanding of these adaptive interactions, they may be able to make more informed interventions in school life. Organisations function through the dynamic interaction of their various parts, so that the whole organisation is greater than the sum of its parts. Some of those interactions are traditionally labelled, 'leadership'. 


\subsection{What is leadership?}

The importance of leadership, as an explanatory idea, is rarely disputed. Leadership is an enduring and far-reaching concept that features in writings from ancient times to the present. Famous people in history are much admired for their leadership accomplishments. For example, the French patriot and martyr, Joan of Arc (c1412-1431), is credited with leading French resistance against the English and so halting the ascendancy of the English in France. A magnificent monument stands in London's Trafalgar Square as a symbol of the leadership of Horatio Nelson (1758$1805)$ in the decisive defeat of a combined French and Spanish fleet ${ }^{1}$. The Turkish general and statesman, Mustafa Kemal Ataturk (1881-1927), is credited with the defeat of British, Australian and New Zealand forces in the First World War invasion of the Gallipoli Peninsula and the later reform and modernisation of the Turkish nation $^{2}$. Caroline Chisholm (1808-1877), an English born social worker and philanthropist, is esteemed for her work in establishing social reforms for women and children in Australia ${ }^{3}$. Nelson Mandela (1918- ) is well-known as a symbol of resistance to the apartheid policies of the then South African government, and later as President of South Africa ${ }^{4}$. Frank Tate (1864-1939) is known for his leadership in reforming the Victorian Education Department in his role as Director of Education from 1902 to 1928 , and his continuing interest in schooling beyond that time ${ }^{5}$. People such as these are commonly identified as leaders and it is generally assumed that leadership of one sort or another will be involved in the establishment or improvement of any organisation.

I argue that the uncritical acceptance of the concepts, leader and leadership, mask a number of significant problems. The leadership concept suffers from problems of identity (how is a leader to be distinguished from a non-leader?), attribution (what are the qualities that distinguish leaders from non-leaders?), and

\footnotetext{
${ }^{1}$ For an opinion about the significance of the Battle of Trafalgar, see Gilbert (2005).

${ }^{2}$ For an opinion about Mustafa Kemal Ataturk, see Byrne (2003).

${ }^{3}$ For a historical commentary about Caroline Chisholm, see Australian Broadcasting Commission (2002).

${ }_{5}^{4}$ A biography of Nelson Mandela may be found in African National Congress (Circa 2004).

${ }^{5}$ For biographical information about Frank Tate, see Wight (2003) and Selleck (1990).
} 
transferability (will a leader in one situation also be a leader in a different situation?). Similarly, the leadership concept is problematic in an ontological sense (is leadership a subjective essence or an objective fact?), in an epistemological sense (what evidence do I need to be able to justify leadership and its effects?), and in a methodological sense (what actions should the 'leader' take to improve this organisation?). Furthermore, the search for solutions to problems like these is constrained by norms of language, culture, and action.

\subsection{Language, culture and thought}

As Vygotsky (1962, p. 51) has said, 'verbal thought is not an innate natural form of behaviour but is determined by a historical-cultural process'. That is, the meanings we create and convey through language arise from the complex processes of social and cultural learning. The words, leader and leadership are theory-laden; they carry with them perceptions and understandings that may exhibit some commonality, but will also display degrees of variation of sense from person to person, from community to community, from culture to culture, and from situation to situation.

Luria (1979, pp. 58-80), also found marked differences in the organisational practices of different cultures. He studied the intellectual activity of people from groups that displayed striking differences in practical activities, modes of communication and cultural outlook. He found distinct qualitative differences in the ways thinking was organised amongst the studied populations. Luria explained these differences in terms of differences in the day-to-day practical activities of people, the reorganisation of activity due to the influence of formal schooling, and sharp changes in social-historical circumstances (such as, for example, changes due to the 1917 Russian Revolution). Human ideas arise in the context of human social life; they are the product of inheritance, environment, life events, and learning. Just as human social life exhibits a complex arrangement of both dissimilar and common characteristics, so too do human ideas and thinking.

The complexity of human communication entails a curious mix of understandings and misunderstandings. The meaning and context of words in language and 
conflicting expectations or conventions in culture combine to render communication indistinct, ambiguous and hazy. The narratives of children's stories, because of their relative simplicity, sometimes portray this type of confusion very efficiently. Take the following example:

"I saw a Heffalump today, Piglet." "What was it doing?" asked Piglet. "Just lumping along," said Christopher Robin. "I don't think it saw me." "I saw one once," said Piglet. "At least I think I did," he said. "Only perhaps it wasn't." "So did I," said Pooh, wondering what a Heffalump was like (A.A. Milne, 1940, p. 54).

In this example, A.A. Milne, portrays a moment of interaction between three characters: a young boy known as Christopher Robin, and two anthropomorphic characters, Piglet and Pooh. A complex interdependency is evident amongst the minds of the characters, the cultural enactment of status and the artefactual properties of the language used. These three characters have different understandings of the word 'Heffalump', yet they are either unwilling or unable to admit this difference. The cultural norm portrayed in Milne's story requires that they talk about Heffalumps in a matter-of-fact way, which disguises the ambiguity and confusion of their communication. This ambiguity is not only due to the apparent haziness of their thinking but also to the structure and properties of the language used. I suspect that similar forms of ambiguity and confusion may be found amongst those who talk about leaders and leadership. In the following chapters, I explain the important dependency of these concepts on culture and language and the problems they pose for leadership research methods that rely largely on survey or interview data.

The subjective interpretations people make of the situations they observe reflect the values and theories they hold, and are the outworking of attention, sense making and memory. That is, they are the outworking of human cognition. In the following example of a response given in a research interview, a teacher holding a formal position of leadership in a school said:

I think everybody here, basically, has this shared goal - this year, more so than last year. Last year it was all over the place, but this year everybody seems to be working together as a team and they see the school operating as a team (Interview B8). 
It is apparent in this comment that this teacher has construed meaning from a particular set of events in her school - meaning that arises from both personal and cultural commitments. In this case, the sense this teacher has made of her situation, refers to a goal towards which she believes she and her colleagues are collectively working. The teacher's account exhibits elements of a first-person report ("I think...; Last year it was...") through which she declares her personal view, and a thirdperson report ("everybody here...; they see...") through which she declares the actions and views of others. Nevertheless, in the final analysis her report is primarily a first-person report, through which she communicates her personal interpretation of events.

Whilst first-person reports may provide useful information about a situation, there is a danger in treating them as the foundation of leadership theory. Evers and Lakomski (1991, pp. 112-136) argue that the interpretations inherent in first-person observation reports can be legitimately queried. The underlying cultural effects, predispositions, and intentions that brought about a particular reading of an event are of crucial importance to the accounts provided by interviewees. For example, in situations that were stereotyped as male domains, female leaders have been viewed less favourably than male leaders when they achieved bad organisational outcomes, yet more favourably than male leaders when they achieved good organisational outcomes (Antonakis et al., 2005).

Whilst methods exist for evaluating the reliability of interview accounts, those methods lose credibility within a foundationalist theory of knowledge, which takes subjective beliefs and interpretations to be the basis of other knowledge claims ${ }^{6}$. However, interview data may provide insight into the culture of organisations if embedded in a larger coherentist framework that includes our best theories, including naturalistic theories concerned with human cognition and learning.

\footnotetext{
${ }^{6}$ When epistemic privilege is given to subjective beliefs and interpretations, as foundational knowledge, obvious difficulties arise when subjects give conflicting accounts. How is one account to be chosen over another except by justifying it and so rendering it invalid as a foundation?
} 


\subsubsection{Culture and learning}

Both human learning and leadership occur in the context of culture and situation. The social, communal nature of human life requires stable norms of practice that bind human experiences together. Cultural norms, whilst enabling the harmonious coexistence of people, also undergo reproduction and evolution through the processes of human learning. It is in times of tension, conflict, need or trouble that certain people may be identified in first-person accounts as leaders. The resolution of such situations requires that problems be understood through processes of action, feedback and learning. Here, I examine the origins of hierarchical leader-follower relations in the production and reproduction of culture.

Our distinctive ability to learn has enabled humans to survive and to shape our environment in a way that distinguishes them from other members of the animal kingdom. In history, humans adapted to change brought about by natural and social causes - changes in climate, natural disasters, cultural differentiation, institutional change, political events, and wars. In the process of this adaptation, the development of socially learned ideas and reciprocal behaviours accompanied the development of cultural artefacts and meanings. Of necessity, the emergence of particular cultures required the assimilation of individuals into the conventions and deeper meanings of particular life styles and outlooks on life. Acculturation was the main activity of child rearing and an important means of improving the survival potential of both individuals and groups. As social groupings adopted technical and social divisions of labour, child rearing underwent a similar transformation and the learning process associated with this transformation became 'education'.

Over time, education practices proceeded from the loosely structured, natural processes of immersion in family and village life to the regulated cultural institution of schooling. Thus Hamilton (1990, p. 12), in his historical overview of the development of education, argues that the rise of schooling required a major reconceptualisation of social learning from the informal transmission of social experience to a 'partitioned and formalised social institution'. Schooling was broken down into a series of tasks, each the responsibility of a different person and subject 
to particular cultural influences. This division of labour, with its associated division of knowledge and expertise, took on a territorial character with associated rights of access and the exclusion of outsiders. As differences in cultural power arose from the uneven distribution of culturally relevant experience or resources, schooling became a social engineering tool, a political means of transforming the whole of society. The emergence of this differentiation may be attributed to changes in the literature of education:

Previously, most educational writers had concerned themselves with the upbringing practices of the powerful sections of society. But from the sixteenth century, a new genre of educational literature appeared: documents prepared by one section of society about the schooling (or disciplining) of their subordinates. Necessarily, this literature embraced a 'top down' view of schooling. The horizontal division of educational labour was visibly recast as a hierarchical or power-based division of labour (Hamilton, 1990, p. 15).

So it was that cultural, economic, and political considerations brought about a changed relationship between education and society. The simple acculturation of children into social life was transformed into the more systematic approach of schooling, which in turn was subject to wider social influences and itself used as a means of transforming society ${ }^{7}$.

Over time, an expanded domain of schooling in education was framed with respect to both curriculum and production. Curriculum embodied a route or journey, a structured course of learning, undertaken to reach a particular educational destination - the achievement of particular forms of learning and intellect, or alternatively, approved forms of knowledge, cultural dispositions and values. If teaching and learning are mediated by curriculum, then power over the curriculum is a means of power over learning. Hence, curriculum takes on the character of a sociopolitical artefact reflecting the cultural selections, values and purposes of groups with the greater social or political influence. Economic forces led to schools being organised as sites of the production and reproduction of economic ways of life within

\footnotetext{
${ }^{7}$ Here, we find the roots of transformational leadership theory, with its emphasis on the hierarchical, power-based influence of leaders on subordinates.
} 
each new generation of adults. The influences of the wider social processes of economic production led to the organisation and reorganisation of schooling and the corresponding classification and reclassification of teaching as a labour process. Such change required attention to the administrative activities of management, monitoring and control. Hence, the prevailing interest in leadership in schools has arisen from a particular sort of power relation - one that is concerned with controlling the processes of enculturation and learning.

In the development of schooling, educational administration aimed to contain, control or channel the actions and reactions of human beings. Consistent with this view, educational administrators were placed in a hierarchical position of social power. They occupied specified positions within a hierarchical organisational structure according to which they performed formal coordination roles. Consequently, educational leadership was associated with division of labour and notions of power and control. By extension, leadership also became a property of the people holding formally defined leadership positions.

The institutionalisation of education led to distinctive forms of organisation in schools. Commonly, schools were structured and organised in ways similar to other socially influential institutions. In the western world, schools adopted recognisable organisational forms such as a partitioned structure, the division of labour, hierarchical systems of authority and control, socially determined notions of production, and cultural norms and values as expressed in formal documents and the common routines of the organisation ${ }^{8}$. In this context, educational leadership was associated with the formal positions of authority, through which the organisation was governed and managed.

\subsubsection{The interdependence of fact and value}

Hierarchical models of control have tended to concentrate on the manipulation and control of a specific school culture by leaders. Cultural politics and hegemonic

\footnotetext{
${ }^{8}$ Callahan (1962) and Bowe \& Ball (1992) provide some examples of the relationship between formal documentation and routine practices in schools.
} 
power relations feature in attempts to manipulate culture in the pursuit of goals such as 'excellence' or 'efficiency' (Bates, 1991). This generally requires the negotiation of values amongst a complex array of mutually complementing and mutually antagonistic viewpoints. Culture comprises both factual and mythical components; whereby the factual components express 'the way things are around here', whilst the mythical components have more to do with the construction of meaning. The mythical component is expressed through metaphor and ritual and whilst it is more open to exploration, reinterpretation and negotiation than the factual component, it tends towards stability rather than variability. It is therefore important to distinguish between the deeply resilient characteristics of an emerging, negotiated culture and the relative superficiality and brittleness of a calculated, imposed organisational culture (Bates, 1991, p59).

Caldwell and Spinks (1991) also acknowledge the problematic nature of cultural influences on the operation of schools. Rather than advocating the manipulation of educational communities by those in authority, they argue for the empowerment of the school administrators, teachers, parents and students who are the members of those communities. They propose that this empowerment be achieved by admitting all members of a school community to collaborative decision-making processes. This view stands at odds with centralised bureaucratic models of bifurcated, hierarchical control with their emphasis on the separation of fact and value.

In contemporary organisation theory, the separation of fact and value may be seen as the outcome of a predominantly Weberian view of rationality:

Weber's view of rationality centres on the claim that statements of fact and judgements of value are logically distinct. (Rizvi, 1986, p. 9)

A rationality of this sort cannot recognise culture in its entirety because culture embodies a world view that has been constructed from a particular construal of fact and value. Regardless of whether the subject of study is physical or social, this form of rationality can only recognise facts. It is dominated by the assumption that values 
cannot be rationally evaluated. Consequently, Rizvi argues that administrative leadership and democratic community are fundamentally incompatible:

If we continue to accept the values represented by the traditional concept of administrative leadership, then we cannot hope to achieve democratic educational administration and to transform schools into democratic communities - goals which have, at least rhetorically, been pursued in recent years in Australian education (Rizvi, 1986, p. 51).

A commitment to democratic communities is a value position that entails a commitment to a particular kind of cultural ideal. As Rizvi has stated, a choice must be made between a commitment to community or the organisation of schools in bureaucratic and hierarchical ways. The former comprises a commitment to democratic values, whilst the latter deemphasises the importance of values in school organisation.

\subsection{The commonsense notion of leadership}

One important characteristic of the democratic ideal, according to Rizvi (1986, pp. 36-40), is its reliance on the meaningful participation of individuals in decisionmaking processes. A commitment to the democratic ideal in institutions is incompatible with the practice of appointing some people as leaders through processes that are primarily autocratic or bureaucratic. Rizvi goes on to say that the social ideal of democratic community, as described by Taylor $(1982)^{9}$, is one that is generally educative, secular and non-hierarchical, and consistent with the desire of all people to participate in decisions that affect them in some way. Taylor argues that three attributes are possessed to lesser or greater degree by all communities. They are:

1. Shared beliefs and values amongst the members of the community;

2. Direct multilateral relations between people (relations that are not controlled or mediated by leaders, organisational hierarchies, or prescribed codes);

\footnotetext{
${ }^{9}$ Rizvi (1986, pp.36-40) argues that this view is consistent with the writings of Rousseau, Marx and Dewey.
} 
3. Reciprocal actions amongst the community members, governed by a range of arrangements, relations and exchanges, including cooperation and sharing.

All of these attributes are compatible with participatory democracies because they entail a certain fraternity or emotional commitment to participation in the life of a community. Whilst these three attributes are mutually supporting, it is shared beliefs and values that lie at the core of a democratic community.

\subsubsection{Folk psychology}

Beliefs and values are attributes of everyday, commonsense theories of mind. These theories or models, which are posits of folk psychology, include explanations for the intentions, beliefs and values held by people. Folk psychological theories feature in interpersonal relations because they purport to explain why we are able to predict the behaviour of others in certain situations, and why it is that we share certain values, beliefs and intentions. Hence, folk psychological explanations account for the ways we commonly make sense of each other's actions and the justifications we give for the decisions we make.

Folk psychological theories attempt to explain a variety of intentional and beliefrelated characteristics of the human mind, including our ability to 'read' the minds of other people. These theories may be classified according to their epistemological roots; that is, in terms of the theories or mechanisms by which we learn and account for what we know. Our supposed ability to attribute particular thoughts and intentions to others and to make accurate predictions concerning the behaviours of others may be classified as being on-line or off-line cognitive activity (Stich and Nichols, 1992).

On-line cognitive activity is that which occurs within a subject and is therefore under his/her direct control. Theory-theories, which refer to a subject drawing inferences from the information he/she has about another person's situation, are therefore labelled as on-line theories. Theory-theorists contend that a subject will 
reach decisions about another person's situation based on his/her existing folk psychological knowledge, that is, based on the posits of a theory.

Off-line activity refers to cognitive activity within a subject, the first-person, which pertains to another subject, the second-person. Since the cognitive work in this case is being done by the first-person, this work is not under the direct control of the second-person and is therefore referred to as off-line cognition. Simulation theories, which refer to a subject drawing on available information to simulate the other person's cognitive processes, are classified as off-line theories. Simulation theorists claim that, rather than utilising theory, the first-person simulates the mental states of the second-person to gain insight into that person's thoughts and intentions (Ribeiro, 2003, p. 1). This view has received some support from neuroscience with the discovery of mirror-neurons (Dobbs, 2006), but not without challenge (Ribeiro, 2003).

Ribeiro (2003) argues that mirror neuron activity can be incorporated into both of the major categories of theories of mind: simulation-theories and theory-theories. Given the research findings at the time her paper was written, she also claimed that motor neurons are only involved in narrowly specific motor actions. However, studies on human subjects undertaken with brain imaging techniques have since revealed that mirror neurons

exist not just in the premotor cortex and the inferior parietal areas but also the posterior parietal lobe, the superior temporal sulcus, and the insula areas that correspond to our abilities to feel empathy, understand intention, and use language (Dobbs, 2006).

Hence, mirror neurons enable us to simulate the experiences of others and to perceive their intentions and emotions. Of course, even with motor actions the ability to copy another person is only the first step towards understanding that person's actions. An initial copy will invariably be imperfect and incomplete and will require further experimentation and refinement. It is at this point that simulation may give way to theorising as the subject attempts to obtain a deeper understanding of the thoughts and intentions of the other person. Hence, it seems that folk-psychology is 
not a question of simulation-theory or theory-theory, but a question of the interaction of processes of simulation and theorising.

Theory-theories and simulation theories may be divided into two further categories: internalist and externalist theories (Stich, 1996, p. 132, fig. 3-1). Internalist and externalist versions of theory-theory are similar to internalist and externalist epistemologies. In both the theory and simulation versions of folk psychology, the internal accounts rely on a description of the inner workings of the mind whilst external accounts rely on a contextually relevant interpretation of an observed situation. Internalist epistemologies take the justification of propositional knowledge ${ }^{10}$ to be a process that is internal to the subject (Steup, 2005). In a similar way, internal accounts of theory-theory connect observable outcomes to a subject's internally represented knowledge structure, which may take the form of schemas or semantic webs. In contrast, externalist epistemologies take the justification of propositional knowledge to be a process that draws on relations outside the subject (Steup, 2005). Like externalist epistemologies, external accounts of theory-theory rely more on cultural relations external to the subject; that is, they focus on the platitudes (shallow statements) made by a subject that are readily recognised and assented to by others.

Simulation theory may also be divided into internalist and externalist categories (Ribeiro, 2003, pp. 7-11). The internal version of simulation theory posits a similarity of internal cognitive mechanisms in both the first-person and the secondperson. Here, the similarity of mechanism enables the subject to simulate the thinking of the second-person. The external version of simulation theory, rather than focusing on internal cognitive mechanisms, seeks to link the other person's actions to the particularities of the situation. Figure 1.1 below incorporates the externalist and

\footnotetext{
${ }^{10}$ Here it is important to distinguish propositional knowledge (knowledge that such and such is the case), from knowing a place or person (a sense of familiarity), or know-how (knowing how to do something). Whilst knowing a place or person, or knowing how to do something may be known tacitly (below the level of consciousness), propositional knowledge is conscious, declarative and objective in character.
} 
internalist versions of simulation-theory and theory-theory into a simple taxonomy of folk-psychological theories.

Figure 1.1 Theories of folk-psychology: a taxonomy

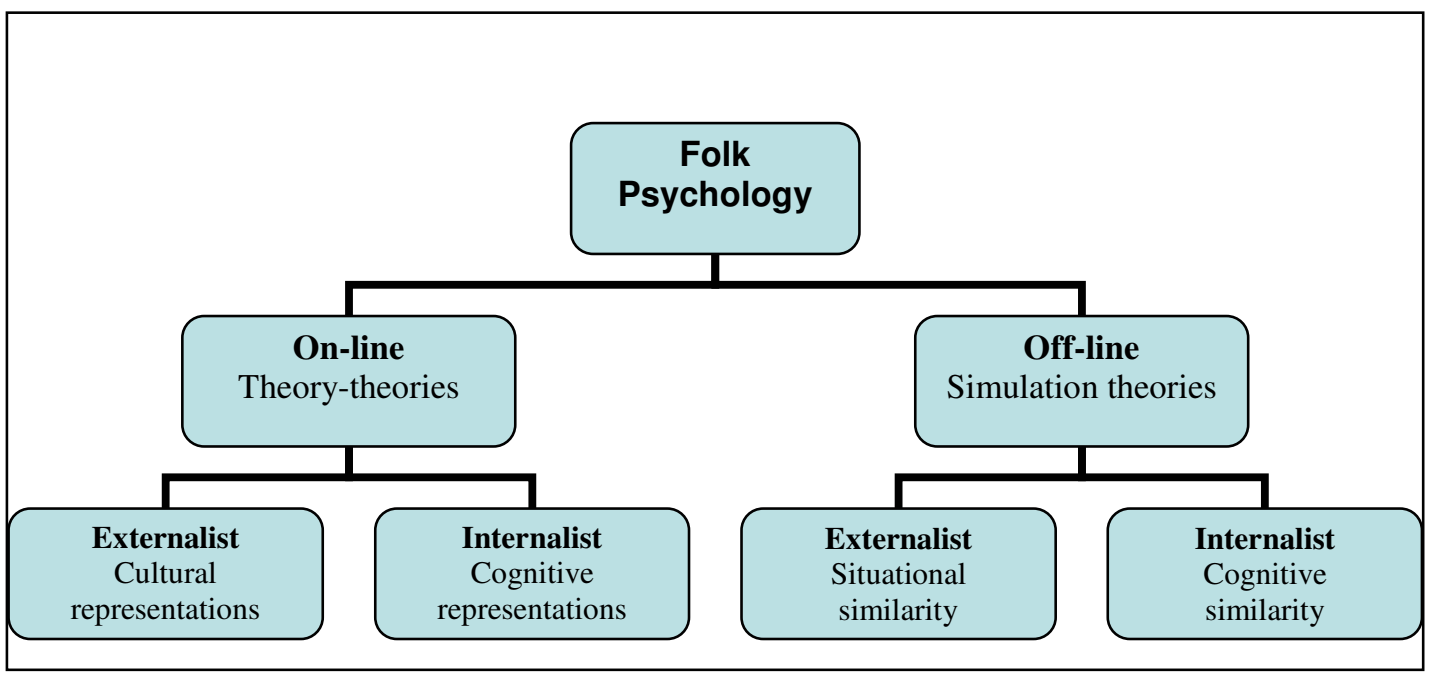

Whilst there are only two varieties of simulation theory presently employed in philosophical debate, many varieties of theory-theory exist. These include eliminative materialism, parallelism, epiphenomenalism, identity theory, nonreductive materialism, metaphysical functionalism, substance dualism and interactionism (Popper \& Eccles, 1983, pp. 51-55; Stich, 1996; Murphy, 2002). Of these theories, eliminative materialism stands apart in predicting the demise of folk psychology altogether, and with it the need for any theory that employs intentionality or belief. The other versions concentrate on explaining intentionality and belief in various ways. Intentionality and belief are important signifiers in narrative accounts.

The commonsense notions of leadership portrayed by folk psychology may be found in leadership narratives. These notions include a sense of emotional commitment to the leader in the form of trust and loyalty. In the following example, non-literal language is used to convey emotional commitment:

"Jackson," Miller said, taking a moment, "why is it you're the only one in the squad that doesn't think I'm a heartless bastard?" "No, sir, I think you're a heartless bastard myself," he said. "That's your job right now, and thank God you're doin' it. And well sir." He smiled at Jackson, 
nodded, edged inside and poked his way through the piled stone and building materials and debris (Collins, 1998, p. 178).

The use of irony by Jackson in this extract conveys a sense of humour that successfully breaks the tension of the moment (evident in Miller's smile, another indicator of emotion). Humour is both emotional and philosophical in nature ${ }^{11}$, and both humour and philosophy can be connected to human cognition and learning.

\subsubsection{Emotion}

According to Carroll (1999, p. 21), the everyday use of the word emotion refers to an assortment of heterogeneous phenomena that cannot necessarily be linked to some sort of natural kind, like gold. He reserves the term feelings for effects that are bodily in nature, and the term emotion for effects that are cognitive in nature. However, other authors instead refer to primary emotions, which refer to innate emotions mediated by the limbic system; and secondary emotions, which constitute the systematic associations mediated by the prefrontal cortex and involve memory and conscious thought (see, for example, Damasio, 1994).

The primary emotions (or 'bodily feelings', if Carroll's terminology is used) are internal bodily events that have no conscious external reference. In contrast to the primary emotions, however, secondary emotions have semantic content because they are directed at someone or something: if I am angry, then I am angry with someone; if I am frustrated, I am frustrated with something. Hence, the secondary emotions are much more than raw bodily responses; they relate to selected features of a situation. Carroll (1999, p. 25) argues that, 'emotions cannot simply be bodily feelings, since sheer bodily feelings lack intentionality'. Intentionality is a category of phenomenology and a feature of the narratives of folk psychology. To connect emotion to intentionality is to describe it in phenomenological terms as the outworking of streams of consciousness.

\footnotetext{
${ }^{11}$ The Austrian philosopher, Ludwig Wittgenstein, once stated that "a serious and good philosophical work could be written that would consist entirely of jokes" (Paulos, 2000, p.2). However, the relevant philosophical point must be understood in order to get the joke.
} 
However, emotion also figures in naturalistic accounts of human cognition, and it is these accounts that have expanded our knowledge of the cognitive processes that involve emotion. Emotions arise from the complex interactions of various cognitive neural modules with other bodily systems, including the somatosensory systems. Emotions organise perception by calling our attention to certain features of a situation, highlighting those characteristics above others. Hence, our emotional states both arise from stimuli in our environments and shape our perceptions of those stimuli. A presiding emotion will guide the formation of our expectations concerning what we should watch for in a given situation.

Emotion cannot be ignored in a complete analysis of leadership. There are two reasons for this stance. Firstly, emotion is deeply manifested in the narratives of folk psychology and is linked to the folk psychological idea of leadership. Leadership, like emotion, is portrayed as a motivating or compulsive force, or as the focusing of attention on an event, goal or task. Secondly, as mentioned earlier, emotions are becoming an increasingly important component of naturalistic theories of human cognition. Since cognitive theory provides our best accounts of human learning and action, it must take a central place in naturalistic theories of organisational functioning.

Damasio (1994), in his book Descartes' Error, gives a detailed account of emotion in human cognition. In doing so, he cites the example of $19^{\text {th }}$ Century railway foreman, Phineas Gage, a person with a reliable and trustworthy disposition. Gage suffered a traumatic accident on a railway construction job. In the accident, a metal rod passed through Gage's head, taking part of his prefrontal cortex with it. The prefrontal cortex is involved in the production of conscious thoughts and emotions $^{12}$. Gage successfully recovered from the accident, with the exception that

\footnotetext{
12 The primary emotions comprise innate, subconscious cognitive processes that depend on the limbic system including the amygdala and anterior cingulate. Secondary emotions are those that arise from conscious deliberation involving the prefrontal cortex (Damasio, 1994, p.133). It was the cognitive functions associated with the secondary emotions that were impaired when Gage's prefrontal cortex was damaged.
} 
he was no longer able to make good decisions and adopted behaviours that were significantly reckless and irresponsible.

Many contemporary medical cases similarly show that loss of function in those brain regions involved in the production of emotional responses result in a loss of cognitive decision-making capability. A scientific analysis of human cognitive capabilities shows, therefore, that human reasoning cannot be divorced from emotion, but depends in a critical way on the cognitive mechanisms associated with emotional responses.

\subsubsection{Informal versus formal theories}

The recognition and interpretation of emotions also figure in the theories of folk psychology. Those theories are expressions of commonsense views of the world, including the intentions and behaviour of other people. As explained earlier, the discovery of mirror neurons adds support to the explanation of folk psychology as off-line simulation. This includes the simulation of situations similar to previously experienced situations (on the externalist reading), or the simulation of the internal cognitive processes of others (on the internalist reading). Whilst the propagation of folk psychology may occur as individuals simulate what they observe in culturally produced situations, folk psychology might take on the form of theory as it is transmitted through language. In saying this, I have blurred the distinction between simulation-theory and theory-theory. Empirical evidence has been found for the firing of mirror neurons in response to actions described in language, so that

\footnotetext{
when people listened to sentences describing actions, the same mirror neurons fired as would have fired had they performed the actions described or witnessed them being performed - a stunning abstraction of a process that would seem to be quite visual and visceral (Dobbs, 2006).
}

The propagation of culturally significant actions may occur through language, whilst the same language may also propagate culturally significant concepts or theories. Hence, language may figure in both simulation-theory and theory-theory 
explanations for folk psychology, insofar as culture and folk psychology are interdependent.

Culturally specific forms of folk psychology are propagated through narrative. These narratives may take the form of rhetoric, legends, myths, biographies, historical accounts, religious texts, and fiction. Narratives may simultaneously describe folk psychological theories and simulate emotional responses in those who witness them as listeners, readers and viewers. The syntactic structure and semantic content of narrative are conveyed through both literal and non-literal language forms, with emotional content being most often found in non-literal expressions. Hence, the persuasive and convincing properties of narrative, which arise from the emotional responses they evoke in both narrators and audiences, cohere with simulation-theory and the action of mirror neurons.

Narrative may be broken down into the two broad categories of situational narrative and grand narrative. Situational narratives are those that portray the particularities of situations whereas grand narratives seek to convey universal principles. Hence, formal theories of leadership may be understood as forms of grand narrative in which general principles have been expressed using formal terms. Whilst situational narratives interpret the actions of people by portraying the folk psychological categories of intention and belief, grand narratives tend to take a more detached, rational or empirical stance. However, as T. B. Greenfield has argued, taking such a detached approach may not be enough if we are to reach a greater understanding of social reality:

The choice we face is whether to interpret reality and all that we recognise as an empirical world by rational methods alone or whether to seek elements of truth also from insight, image, art, and all the ways of knowing that rely upon intuitive, self-oriented, and non-rational perception (T. B. Greenfield, 1980, p. 37).

Two questions remain, however. Firstly, if we choose rational methods alone, how are we to deal with the limitations of language and the problem of the interpretation and communication of meaning? Secondly, if we choose intuitive 
methods, how are we to convey the inexpressible, subjective understandings that comprise insight and non-rational perception? Language is a human artefact that both expands and limits our ability to understand the realities we perceive. It is also acts as both the product and source of culturally shared concepts. The difficulties in the first question, concerning meaning, are highlighted by the problem of self-reference.

\subsection{Self-reference in formal theories of leadership}

Leadership is a cultural concept that finds its place in both situational narrative and formal theories. Yet as a theoretical term, it has been difficult to pin down. As Foster has written,

Leadership is a construct which must be rebuilt. The dismantling is necessary because it would appear that the future of leadership studies in social science research is bleak (Foster, 1986, p. 3).

The leadership construct has indeed been rebuilt - many times. The huge array of publications about leaders and leadership, and the many varieties of leadership theory are testimony to this occurrence. Despite the ongoing activity of leadership writers, theorists and consultants over past decades, the future of the concept of leadership remains in question (Pfeffer, 1977, Sashkin and Garland, 1979; Bresnen, 1995; Bryman, 1997; Alvesson and Sveningsson, 2003; Lakomski, 2005; Reger, 2007). One important difficulty for formal theories of leadership lies in our attempts to reduce leadership to a set of general principles. The communication of theories requires that they be formalised in theoretical propositions. However, the weakness of formal systems is that they are either inconsistent or contain truths that are unprovable.

\subsubsection{The logical paradox of self-reference}

Consider the following sentence:

Be spontaneous! 
Being spontaneous implies acting in an unprompted way, yet acting in response to the command would qualify as a prompted action. On a literal reading, this command is paradoxical, because if it is obeyed, it is simultaneously disobeyed. Statements such as this one can be employed successfully in humorous narratives because they are either inconsistent or incomplete. If the above example is a complete statement of affairs, then it is inconsistent. If it is consistent, then it must entail the communication of a truth not contained in the statement itself, and is therefore incomplete. This statement is one example of the paradoxes that permeate all formal systems or theories, which rely on literal expressions in language.

Unresolvable self-referential paradoxes, contradictions or antinomies exist in any formal system. Consequently, all formal systems are either inconsistent or incomplete. They are therefore incapable of proving or disproving particular kinds of truths (Paulos, 2000, p. 45). In classical logic, anything can follow from a contradiction and a formal system can contain an infinite number of contradictions. Even if one does not subscribe to classical logic, the existence of contradictions in a formal theory will require some form of justification. It also follows that a consistent formal theory will be incapable of explaining all the facts that arise within it.

Since all formal theories are affected by the problem of self-reference, it follows that all those theories that describe leadership in formal terms are similarly affected. At first glance, it may appear that this is not necessarily the case for leadership events observed from 'outside' particular situations. In these cases, an 'external' observer is not external to the situation at all, but acts as an internal observer within an expanded frame of reference. It is not possible to observe an organisation, even superficially, without having some effect on it and therefore becoming part of a wider system of interpretation. This effect is accentuated when an observer penetrates more deeply into an organisation with the aim of getting at deeper truths. Consequently, the self-reference problem continues to apply even when organisations are purportedly observed 'externally'.

If an historian, for example, sets out to analyse the leadership of Henry $\mathrm{V}$ at the Battle of Agincourt in 1415, he does so as an external observer. After reviewing 
various historical accounts, he might reach conclusions about the use of weapons or tactics, the organisation of the armies that took part in the battle, or the execution of prisoners; and link these in some way to the actions of Henry V as the English leader. Though he will inevitably draw on his preferred ideas and theories in interpreting and explaining the actions of Henry, he is after all looking at a specific situation in History, and might resist the temptation to generalise from this single situation. At first glance, there does not seem to be a problem of self-reference here.

It is a different matter, however, if the historian sets out to discover or prescribe a set of general principles of leadership, common to all people and [leadership] events. The difficulty arises from his membership of the set of all people. Similarly, any [leadership] event of which he is a part, or which he uses as a model, will be a member of the set of all [leadership] events. Consequently, any attempt to formulate general principles for leadership can involve self-reference. In the example of the analysis of Henry's actions at the battle of Agincourt, both Henry and the historian are members of the set of all people in history. The problem of self-reference appears whenever the historian attempts to draw any general conclusions about leadership from a single situation in history. Even if the historian seeks to draw general conclusions from a multitude or set of situations the problem remains, but also suffers from the problem of induction (which is discussed in Chapter 2).

\subsubsection{Inconsistency or incompleteness?}

In 1910, starting with a problem of self-reference in arithmetic, the mathematician, Kurt Gödel, proved that any logically formal system is either inconsistent (contradictory) or incomplete ${ }^{13}$. As described earlier, these problems place severe limitations on formal theories. The problems of self-reference, for which Gödel's theorem applies, arise from within language-based, symbol systems. Selfreference is not always an obstacle, however. It can be quite helpful in solving quite

\footnotetext{
${ }^{13}$ The problems of incompleteness and undecideability arise from cases of self-reference and are also described in the theorems of Solomonoff, Kolmogorov, Turing, Church and Chaitin (See Chaitin, 1999 for an overview of incompleteness, uncomputability and algorithmic information theory).
} 
difficult problems through the process of mathematical or computational recursion ${ }^{14}$, or in the knowledge representations created through parallel distributed processing $(\mathrm{PDP})^{15}$. In the nervous systems of humans, PDP processing may take place at various levels:

- in protein crystals in the regions between nerve cells (synapses),

- in the interactions of nerve cells (neurons),

- in the interaction of smaller networks of neurons,

- in the interactions of regions of the brain and body,

- in the interactions of the minds of people, and

- in the large scale interactions of cultures.

Regardless of their size and complexity, these systems exhibit the problem of incompleteness or undecideability because they are described in formal terms. In later chapters, I explain the mechanisms of PDP in more detail and extend this idea to organisations in processes of distributed cognition, which is described in causalcomputational terms. If intentional terms can be successfully admitted, then distributed cognition may give rise to a theory of distributed agency, of which distributed leadership is a special case.

\subsection{Leadership and distributed social action}

The concept of leadership as a distributed phenomenon - one that is shared amongst the members of an organisation - has received increased attention from researchers in recent years (see Spillane et al., 2001, Spillane, 2006, Spillane et. al, 2007; Gronn, 2000, 2002a, 2000b, 2003, 2006; Harris, 2004, 2005a, 2005b, 2006). According to Gronn (2002a), the idea of distributed leadership was first raised by the late Australian leadership theorist C. A. Gibb (1913-1994) and later revived by

\footnotetext{
${ }^{14}$ Recursion is simply the repeated use of feedback loops in an information processing system. It is an iterative process by which the outcome of a calculation is fed back into a computation as a new input and the process repeated until some predetermined limits to the computation have been reached.

${ }^{15}$ Parallel distributed processing (PDP) occurs in the nervous systems of humans and animals, and is simulated in artificial neural networks. Both natural and artificial forms of PDP utilise recursion through feedback loops.
} 
Brown and Hosking (1986). The term, distributed leadership, is sometimes used in a functional sense as a reference to the conscious delegation of organisational tasks by leaders. However, in this thesis, the term distributed leadership, refers to a special case of distributed agency, which is in turn is derived from distributed cognition theory, a naturalised theory of human cognition. Distributed cognition theory acknowledges the widely interactive and boundless perspective of social activity and human learning (Hutchins, 1995; Clark, 1997; Strauss \& Quinn, 1997, List, 2005). In the context of an organisation, organisational improvement occurs through human learning; that is, through the evolution, or revolution, of human action and theoretical understanding. According to distributed cognition theory, human action and learning is mediated by the complex interaction of physical structures, artefacts and technologies, socio-cultural norms, and the learning of its individual human members.

\subsubsection{Leadership and learning}

The concepts of leadership and distributed cognition both obtain their meaning within socio-cultural domains. Distributed leadership combines leadership and distributed cognition together into a single socio-cultural concept. Whilst distributed cognition theory has roots in the discoveries of neuroscience, the origins of the concept of leadership lie in folk psychology (Lakomski, 2005, p.73). The former describes learning naturalistically in terms of physical and computational processes, whilst the latter describes leadership phenomenologically in terms of human intuition and intentionality. Distributed leadership, as it is conceived in this thesis, is a special case of distributed agency. Human agency is a manifestation of intentionality, which is a category of phenomenology related to consciousness, intuition and free-will (Levinas, 1973; Nichols, 2006). Distributed agency and distributed cognition, therefore, are concepts that combine causal and computational processes with intentional and intuitive qualities. In taking this position, I have blurred the distinction between naturalistic and phenomenological world views.

The term 'distributed leadership', has been coined for diverse purposes in the research literature (Bennett et al., 2003). Some writers appear to use the term as little 
more than an alternative label for familiar notions of collegiality, delegation or democratic decision-making. Whilst there does seem to be tacit agreement that distributed leadership describes a property emerging from a group or network of interacting individuals, significant differences are evident in the explicit uses of the term. Spillane et al. (2001) have based their use of the term 'distributed leadership' on distributed cognition theory, which seeks to provide a coherent explanation of human cognitive processes. Other references to 'distributed leadership' have used the term in a functional sense to describe a decentralised form of organisational structure and power relations (see MIT Sloan, 2005; Harris, 2004; Harris, 2005a; Massachusetts Department of Education, 2004). Another approach has been to describe distributed leadership as a form of concerted action that renders followership redundant (Gronn, 2002a).

Given the relatively recent rise of distributed leadership, it is not surprising to find that critical reviews of that concept are rare. Lakomski (2002, 2005), however, finds the hybrid idea of distributed leadership both helpful and inadequate; helpful in that it provides a beneficial shift away from a preoccupation with the roles of 'designated positional leaders'; inadequate in that matters of coordination or harmonisation of organisational action do not, as a matter of necessity, require the label 'leadership'. The concept of leadership proceeds towards obsolescence as it describes a quality that is increasingly dispersed within organisations.

\subsubsection{Distributed cognition}

Distributed cognition theory has the potential to provide a comprehensive and powerful theory of learning, simply because it takes a holistic perspective that need not exclude any theoretical or empirical analysis. It has gained increasing attention through work of researchers such as Rumelhart, McClelland and the PDP Research Group (1986), Lave and Wenger (1991), Hutchins (1995), Clark (1997), and Strauss and Quinn (1997). A large variety of leadership theories are presented in the research literature, as well as in popular organisational publications and parlance. Few of them, however, make direct reference to developments in our knowledge of human cognition. 
Much of the discourse concerning human cognition focuses on brain function because the brain is seen to be the locus of cognition. The brain is composed of vastly interconnected nerve cells, amongst which information processing is shared or distributed, and is connected to the body through a vast network of nerves and electrochemical processes. The body is also connected to the environment via various sensory pathways. Since the environment inhabited by humans is usually a social environment as much as a physical one, the cognitive processing of information becomes distributed amongst the people, artefacts and natural objects of a given situation (Hutchins, 1995). Hence, cognition is not synonymous with brain function. It arises from complex processes that crucially involve the brain, but which are not due to the brain alone. Protein crystals, synapses, neurons, brain regions, brain, body, person, organisation, culture, environment, and world represent successive, interdependent levels of function. Multiple levels of function require multiple levels of analysis.

\subsection{Levels of analysis}

In this study, multiple levels of analysis are utilised. Here, learning is conceptualised according to a micro-meso-macro triumvirate that may be focused on a particular level of analysis, but also takes adjacent higher and lower levels of function into account ${ }^{16}$. For example, learning processes may be analysed at a microlevel in the biochemical and electrochemical processes of the human brain, at a meso-level in the interactions of the brain with other parts of the body, and at macrolevel through the interactions of humans with one another and their environment. Causal relations and feedback loops may exist within levels, and perhaps also between levels. If an appropriate level of analysis is chosen, it may be possible to achieve a satisfactory analysis of a particular learning situation. However, utilising one level of analysis at the exclusion of others leaves open the possibility of causally relevant phenomena being omitted from theoretical analyses, resulting in a theory deficient in explanatory power. A comprehensive, justifiable and sustainable theory of learning should incorporate all of the significant levels of analysis. Similarly,

\footnotetext{
${ }^{16}$ The micro-meso-macro framework used here is based on ideas presented in Lewis (1992).
} 
organisational changes may be analysed at a micro-level in classrooms, a meso-level in schools and a macro-level in school systems.

In the industrialised world, models of school organisation have undergone several major policy shifts over the past two centuries. For example, Caldwell and Hayward (1998) in their book The Future of Schools, describe the system-wide decentralisation of the Victorian school system under Don Hayward, Minister for Education in the 1990s. Caldwell and Hayward (1998) argue that the transformation of public schooling requires 'extraordinary leadership.' Their view of leadership fits into the transformational leadership model:

Leadership is a process that involves setting a new direction or creating a new vision, and the building of commitment to moving in that direction or achieving that vision (Caldwell and Hayward, 1998, p. 164)

A process of alignment is central to their view of leadership. Here, alignment with a particular view of education and educational administration is sought amongst government, bureaucracies, unions and professional organisations, as well as principals and teachers in schools. Caldwell and Hayward (1998) frame this process as both the conscious and deliberate implementation of policy by the exercise of management power and as a process that is a socio-political process that is shared amongst stakeholders. In the closing chapter of their book, they highlight the importance of learning from past reforms when shaping future policies and actions:

We have learned that reform must be comprehensive and coherent and that single initiatives rarely deliver the range of benefits that are sought (Caldwell and Hayward, 1998, p. 174).

Caldwell and Hayward (1998) recognise that the transformation of educational practices requires causal action within and between the various levels at play in an educational setting. In this case, the educational setting they are referring to is the Victorian government education system. They also recognise that, in a public school system, successful reform requires the cooperation of the educational bureaucracy, principals and other school leaders, and teachers; and that the focus of this cooperation is student learning. In this account, they join a conscious, rational, and 
reflective form of learning to transformational leadership, insisting that the reform of public education requires ‘extraordinary leadership' (1998, p. 164).

Associations between learning and leadership in educational settings may be made in three ways. Firstly, those who typically perform leadership roles in schools are expected to have an adequate knowledge of the processes of learning. Secondly, leadership is traditionally framed in terms of hierarchical control in the administration of schools. It differs in significant ways from the relations between teachers and students (even though teachers are sometimes portrayed as the inspirational leaders or guides of their students). The transition from teacher to educational administrator, and any subsequent changes in school policy or practice, requires learning on the part of the administrator and of the other members of the school; that is, learning must occur collectively in the socio-political relationships of the organisation as new patterns of action are created.

Thirdly, learning and leadership may be viewed as socially interdependent phenomena. Hence, leadership occurs together with learning as part of the sociopolitical interactions of individuals and the organisational environment of schools. In its full realisation, this viewpoint enables an examination of leadership practices at all levels of the organisation, and not only as practised by those assigned formal roles of authority. This is in agreement with Foster's (1986, p. 3) view of leadership as a transient, temporal activity that can be practised by different social players at different times depending on the circumstances and the individual's strength of ideas. Leadership, like learning, may be analysed on a number of levels, including the individual (micro-level) leadership relations of individuals, the organisational (mesolevel) leadership relations that occur amongst and between groups of people, and the wider socio-political (macro-level) leadership effects that occur as people in schools interact with other members of society.

With leadership viewed as a special case of learning and cognition, should leadership theory be subsumed into a theory of organisational learning? Lakomski (1999, p. 1) argues that the little we do know about leadership is contradictory and inconclusive and leadership may, in the final analysis, be an epistemologically 
unsustainable concept. Evers and Lakomski (2000, pp. 70-71) also argue that the concept of leadership is 'causally massively disconnected from the world'. According to Evers (2000a), rather than seeking to uncover the essence of leadership, or explaining organisational functioning in terms of theories of leadership, a better way is to examine the role of learning and the collective actions of organisational members. Whereas essence is a concept of phenomenology, learning and organisational functioning may be framed as a causal-computational process, which is amenable to naturalistic analysis.

\subsection{Naturalistic coherentism}

The naturalistic coherentist epistemology of Evers and Lakomski (1991, 1996, 2000 pp. 141-155) is utilised in this thesis as a means of explaining learning and organisational functioning. Naturalistic coherentism blurs the distinction between the natural and social sciences.

Naturalistic coherentism adopts a theoretical position consistent with our best knowledge of human learning and is therefore suited to an investigation of learning in organisations. This approach is holistic in the sense that it seeks the best global account of the object of study and the 'most encompassing and parsimonious theory possible' (Donmoyer, 2001). In doing so, it puts aside any demarcation claims between the disciplines included in that account (Allix, 2003). Hence, naturalistic coherentism employs a form of methodological holism (Healey, 2004). It seeks to explain complex systems, such as organisations, in terms of the properties of the whole, rather than by explaining the structure and behaviour of its component parts alone.

Knowledge of the fine-grained micro processes of an organisation is necessary if internal causal effects are to be identified. However, a holistic analysis acknowledges that the properties of the whole may not be entirely determined by the properties of its component parts. Another variety of holism known as metaphysical holism (Healey, 2004) holds that a system cannot be explained entirely in terms of supervenience relations, according to which high level properties are determined by 
the properties of component parts at lower levels. According to metaphysical holism, a complete causal account cannot be obtained simply by explaining the functions of the component parts and their effects on higher levels of the system. In this thesis, I allow for metaphysical holism by admitting the possibility of phenomenological accounts, but primarily seek to explain organisational functioning naturalistically by employing methodological holism.

A holistic investigation requires a recursive methodology, which employs progressive cycles of feedback. In other words, to achieve growth in knowledge, repeated spirals of analysis, problem specification and resolution are employed according to a set of soft constraints: simplicity, consistency, comprehensiveness, explanatory unity, fecundity, and learnability. Figure 1.2 represents this spiral schematically.

\section{Figure 1.2 Recursive growth in knowledge}

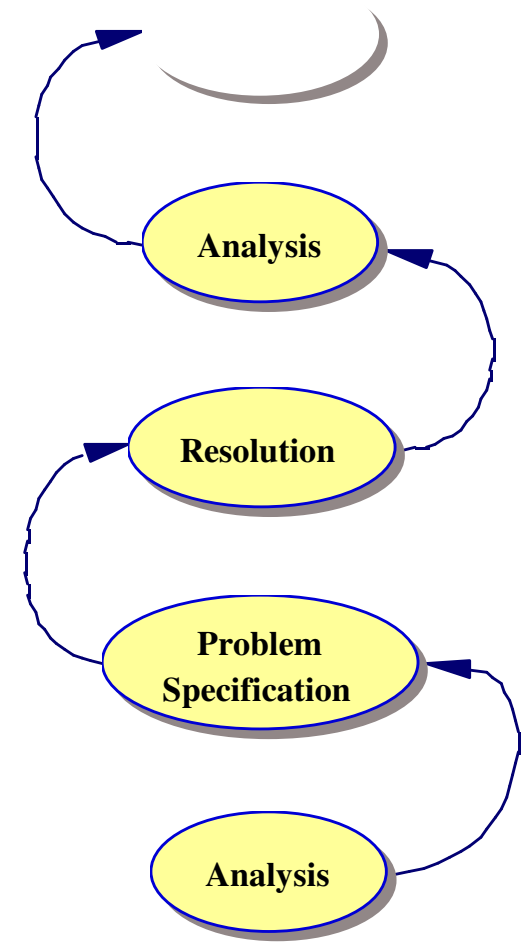

In this model, the problem for investigation is specified after analysis of the current state-of-play. A resolution of the problem is formulated and this resolution 
becomes the new state-of-play. The new state-of-play consequently undergoes analysis so that the spiral can continue upward towards better and better theoretical resolutions.

The current 'state-of-play' of naturalistic theories of learning draw on a number of disciplines, which include neuroscience, parallel distributed processing and information theory ${ }^{17}$ :

- Neuroscience is the scientific study of the structure and function of the biological systems of communication and control, including the central and peripheral nervous systems and the endocrine system, and the study of behaviour and learning.

- Parallel distributed processing $(\boldsymbol{P D P})^{18}$, also known as connectionism, is the study of high-level cognitive activities as practised in computer science, cognitive psychology and sociology. When applied at a higher level to organisations and other social settings, PDP becomes distributed cognition.

- Information theory is the mathematical theory of data communication and storage. It includes cybernetics (Wiener, 1948), which applies information theory to feedback and control processes in dynamic systems.

For the purposes of this study, these disciplines are differentiated as levels of study. Here, neuroscience explains the micro-level characteristics of human cognition, that is, the structure and function of the various parts of the nervous system (e.g. neurons). PDP explains meso-level characteristics, that is, the relations that enable a cognitive system to process information (e.g. relations between neurons, between brain and body, between body and situation). Information theory explains macro-level characteristics, that is, the immaterial form of information and its statistical relationship to matter (in terms of randomness and order). Figure 1.3 (below) shows one example of a three-level analysis of distributed cognition. This

\footnotetext{
${ }^{17}$ For a more comprehensive account of these developments see, for example, Damasio (1994), Churchland, P. S. and Sejnowski, T. J. (1992), Lave and Wenger (1991), Strauss and Quinn (1997), Rumelhart, McClelland and the PDP Research Group (1986), Hutchins (1995).

${ }^{18}$ See Rumelhart, McClelland and the PDP Research Group (1986) for further information about PDP.
} 
version shows the relations between the three fields described above according to their contributions to distributed cognition theory. Together, these fields are making important contributions to our understanding of human learning as a distributed, emergent process.

Figure 1.3 Three levels of analysis for distributed cognition.
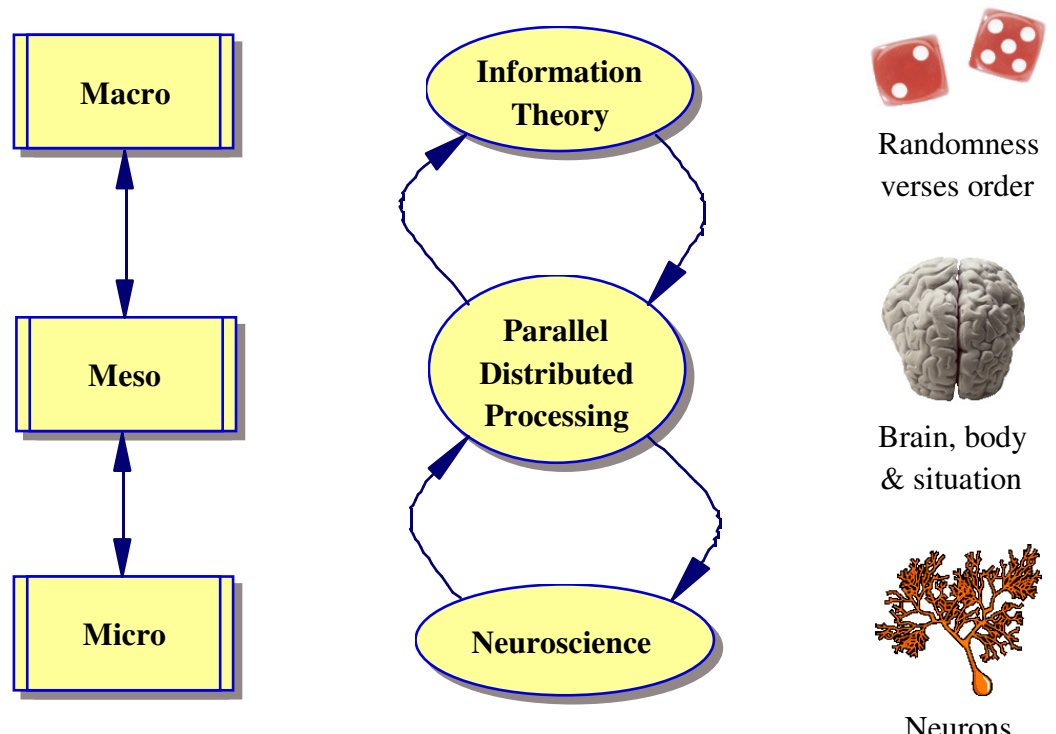

\subsubsection{Methodological considerations}

The relations between these three levels may be understood as vertical relations in accordance with the principle of emergence, by which high level, complex phenomena or patterns arise from simple, lower level processes. The high level, complex phenomena may also be understood to be supervening on the lower level processes. As stated earlier, according to metaphysical holism, emergence and supervenience relations cannot offer complete explanations, leaving room for phenomenological explanations. Nevertheless, in a naturalistic analysis, high level outcomes may emerge from low level events. For example, the 'wetness' of water is an emergent property that arises from the electrostatic attraction between water molecules and their collective attraction to hydrophilic (or 'water-loving') surfaces. Emergent properties cannot be predicted from, or reduced to, lower level descriptions. Emergence is evident, for example, in the distinct properties of atoms 
that arise from the quantum fuzziness (uncertainties) of electron behaviour. Similarly, organisational behaviours emerge from the individual behaviours and interactions of an organisation's members.

Whilst the principle of emergence describes the upward correlation of lower level descriptions with higher level patterns, the principle of supervenience describes the downward correlation of a higher level system to rules or descriptions at a lower level (See figure 1.4 below). A particular case of supervenience is understood to correspond to a unique situation at a lower level, ${ }^{19}$ even though it cannot necessarily be derived from such a situation (Mulhauser, 2005). Supervenience, like entailment, is reflexive, transitive and non-symmetric. However, supervenience is generally not sufficient for entailment. A general pattern may logically supervene on particular facts even though the particular facts do not entail that general pattern (McLaughlin and Bennett, 2005).

\section{Figure 1.4 Emergent and supervenient relations}

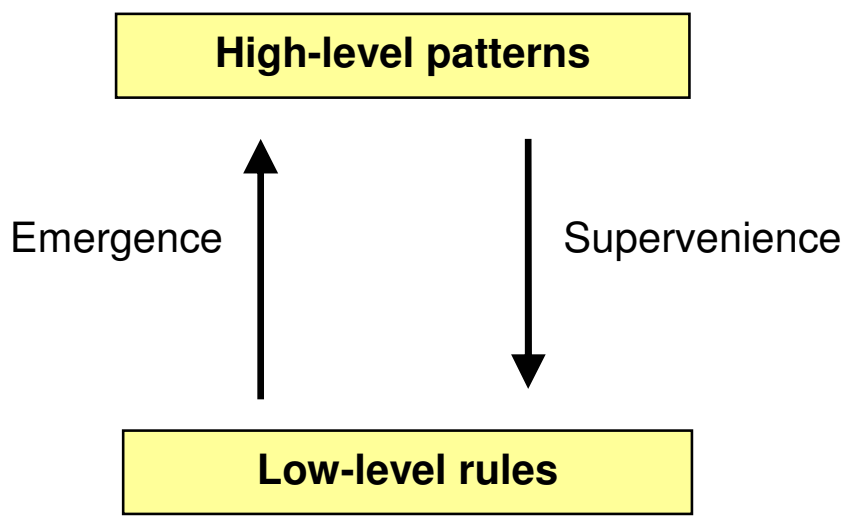

A multi-level analysis that entails emergence and supervenience relations requires a methodology that employs multiple methods. The methodology employed in this study is organised according to the three worlds of inquiry of Popper and

\footnotetext{
${ }^{19}$ For example, only one painting may be the genuine 'Mona Lisa'. Being the 'Mona Lisa' supervenes on the particular structure and properties of that painting and the particular set of circumstances that led to the painting being created. Similarly, the identity of a water molecule supervenes on the bonding of hydrogen and oxygen atoms in the ratio $2: 1$.
} 
Eccles (1983). In this model, the theoretical world includes theoretical objects such as the Australian parliamentary system, Beethoven's Ninth Symphony, Pythagoras' Theorem or the General Theory of Relativity. The theoretical world is distinguished from the subjective world of mind, which includes the folk-psychological categories of intention, belief and hope; and the objective real world, which comprises the physical objects of time, space and energy. Insofar as theories are held in minds or stored on some sort of material, and minds are centred on brains, the distinction between these three worlds is blurred. In certain respects, the theoretical world supervenes on the subjective world, which in turn, supervenes on the physical world. Similarly, mind may be understood as an emergent property of the physical world, and theories as emergent properties of minds.

The methods employed in this study include methods that may be located in the theoretical world, which include philosophical and methodological triangulation and the theoretical analysis of theories of leadership, learning and organisations. They also include methods that may be located in the subjective world, which include heuristic search strategies and the hermeneutic analysis of narrative. Together, these methods are employed to explain the nature of organisations and learning and the place of phenomenal accounts of leadership.

\subsection{Distributed leadership}

A complete account of the functioning of organisations will be one that explains the impact of physical environment on organisations, the cultural influences on human interaction, and the cognitive capabilities of humans. However, whilst organisational functioning and human cognition can be explained naturalistically, that is not the case for leadership. Distributed cognition theory enables organisation theory to be joined to cognitive theory to explain organisational learning. Leadership, however, resists naturalistic description for a number of reasons. Firstly, the leadership agenda is usually associated with 'diverse, anomalous and extraordinary tasks' (Evers, 2000a, p. 243), whilst naturalistic explanations typically depend on statistically stable, routine patterns of organisational action. Secondly, as a relational property that depends significantly on the particular situations in which leadership is 
to be exercised, leadership resists general description. Thirdly, the leadership concept depends on categories of intentionality, which are categories of phenomenology. Hence, to find leadership is to enact a phenomenological reduction, which on a Husserlian reading of phenomenology, purifies 'concrete life from any naturalistic interpretation of its existence' (Levinas, 1973, p. 93). No phenomenal property can be identical to a physical property (Nida-Rümelin, 2006). Hence, a phenomenal property of organisational functioning (such as leadership) cannot also be described as physical property of organisational functioning. Therefore, to theorise about leadership in organisations conceived naturalistically requires a form of property dualism.

Distributed cognition relies on a naturalistic causal-computational explanation of learning, which explains organisational functioning in terms of the collective cognitive contributions of organisational members. These collective contributions cannot be justified as manifestations of leadership without also enacting a phenomenological ontology. To do so is to enact property dualism so that distributed cognition is described according to naturalistic concepts and leadership is described according to phenomenological concepts. This, in turn, enables a naturalistic distributed cognition theory to be joined to leadership as a form of agency. Hence, leadership becomes a special case of distributed agency, which is here called distributed leadership. Hence, the theory of distributed leadership proposed in this thesis describes cognition in formal causal-computational terms, whilst also describing leadership using informal, intentional terms.

Distributed leadership may be understood as a special case of human agency, where agency is enacted through collective, concerted action and learning. Such a theory should be able to explain the formation of resilient organisational practices, the self-organisation of groups within organisations, and the role of individuals in the emergence of new patterns of practice.

The study of learning and leadership has theoretical and practical consequences for teacher education, the organisation of schools, and educational research. As Evers (2000b) explains, a composite view of leadership and learning, in particular 
organisational learning, may lead to an organisational design that is appropriate to the particular needs of schools.

The practical pay-off for good theorising can be a workable organisational design for schools that maximises the purported advantages of leadership in decision-making, while using the error correcting, or learning, resources of the whole organisation (Evers, 2000b, p. 221).

A sustainable view of organisational functioning in schools will be one that coheres with our best current knowledge of human cognition and provides specific solutions to problems of educational practice.

According to Fullan (1993, pp. 39-40), every person within an organisation is a change agent, not simply those formally identified as leaders. Evers and Lakomski (1995) argue for an 'educative' approach to organisational improvement that recognises the need for both individual and organisational learning. Organisational learning occurs when the members of an organisation collectively acquire new knowledge, understanding, know-how, techniques, or practices in a way that changes their mental images of the organisation (Argyris and Schön, 1996, p. 16).

Organisations may be understood in two different ways: as entities with an objective physical reality, or as contexts of human action, in which people act according to their subjective understandings (Greenfield, 1980). Hence, Greenfield contends that individuals live, know and learn, not organisations. Evers and Lakomski (1991, p. 95) are firmly in the objective reality camp, viewing organisations as real objects encoded in the central nervous systems of human beings; with their reality being equivalent to the reality of physical objects such as tables and chairs ${ }^{20}$.

What is the most useful view of organisations in a study of leadership? Popper (1972, p. 341) agrees that all social phenomena should be analysed in terms of the

\footnotetext{
${ }^{20}$ It may also be argued, putting the issue of consciousness aside for a moment, that human beings are simply mini-organisations themselves - that is, self-organised, dynamic assemblages of body tissue through which matter, energy and information flow.
} 
actions and reactions of individuals. Argyris and Schön (1984, p. 360) describe organisations as political entities, agencies, task systems, theories of action, cognitive enterprises and artefacts, and processes of reflexive inquiry. Starratt (1994) sees organisations as social arrangements that are artificially constructed and structured to benefit some segments of society at the expense of others. The organisation as object - organisation as social construct dichotomy limits rather than assists our knowledge of organisations. A better way, in my view, is to acknowledge that organisations may be viewed both objectively and subjectively.

\subsection{Synopsis of the following chapters}

In the following chapters of this thesis, a coherent, naturalistic account of organisational learning is constructed. The first-person, phenomenological perceptions of practitioners are also acknowledged, as parallel narratives of organisational life in schools. The phenomenological essence of leadership, as perceived by practitioners in their day-to-day work, may be placed alongside a holistic, naturalistic account of the causal-computational processes of distributed cognition. Phenomenological accounts, on their own, lack explanatory power, but may provide intersubjective access to the sense making of organisational participants. The naturalistic account examines the tacit and explicit forms of human knowledge and organisational learning. As this naturalistic account combines social science with natural science in ways that avoid the extremes of interpretivism and logical empiricism, it may bring about a coherent explanation of organisational life.

Chapter 2 describes the epistemology adopted in this study. Here I give an account of naturalistic coherentism, as it is employed in this thesis, and compare it with other theories of knowledge.

Chapter 3 builds on the discussion in Chapter 2 to describe the methodology adopted for this study of distributed leadership in schools.

Chapter 4 presents narratives of leadership as depictions of folk psychology. This includes an analysis of the interpretive schemas employed in narrative. 
Chapter 5 analyses the attribution of meaning in narrative. In doing so, it examines the development of theories of mind and their relation to folk psychology.

Chapter 6 analyses formal leadership theories and their contribution to theories of organisational functioning.

Chapter 7 introduces distributed cognition theory. Its status as a theory of information processing, knowledge and learning, and its relevance to leadership theory, is discussed.

Chapter 8 reviews the preceding chapters and concludes with a discussion of distributed leadership in schools. 


\section{Chapter 2: Epistemological considerations}

This chapter evaluates various epistemological positions and explains the theory of knowledge utilised in this thesis. Here, appeals to authoritative or foundational knowledge are rejected in favour of a naturalistic coherentist theory of knowledge. This epistemology draws on scientific knowledge about human learning and the emergence of organisational behaviour. It also evaluates theories in relation to other trusted theories, using criteria such as conservatism, simplicity, internal consistency, evidential support, and explanatory power. Knowledge, thus conceived, comprises an interdependent web of theory and observation, which does not privilege observation over theory.

\subsection{Introduction}

Despite the intense and persistent interest shown by researchers and leadership commentators, leadership remains a perplexing and ambiguous concept. As Bennis (1959) has said,

of all the hazy and confounding areas in social psychology, leadership theory undoubtedly contends for top nomination (Bennis, 1959, p. 259).

The ambiguity of leadership (Pfeffer, 1977) arises, in part, from problems of epistemology. In this chapter, I describe a theory of knowledge suited to the naturalistic analysis of schools.

Leadership theories may formally attribute relationships of cause and effect to leadership in organisations ${ }^{21}$, where the terms, 'cause and effect', bear a close resemblance to Aristotle's concept of efficient cause $e^{22}$. Such theories imply that leadership is an object of nature, which entails certain causal properties (see for example, Leithwood et al., 2006). However, leadership may also be viewed as a

\footnotetext{
${ }^{21}$ See Kuo (2004), Jago (1982), Weber et al. (2001), and Snowden (2007) for examples of causal explanations for leadership.

22 Aristotle's concept of efficient cause is discussed later in Chapter 6, Sub-section 6.1.2. See Kuo (2004), Jago (1982), Weber et al. (2001), and Snowden (2007) for examples of causal explanations for leadership.
} 
phenomenological essence, evident in the beliefs, values and intentions of subjects to which it is associated. The epistemological problem is that these qualities cannot satisfactorily be described in causal terms.

Whilst the performance of schools can be described in causal-computational terms according to theories of learning and theories of organisational functioning, the phenomenological qualities of leadership cannot. Attempts to naturalise the phenomenal experience of leadership are undermined by the inherent ambiguity of phenomenal perception. This ambiguity arises from the plurality of phenomenal content in any leadership experience.

Phenomenal content may occur in at least three ways. Firstly,

there may not be such a thing as the representational content of a perceptual experience. Instead, a given experience may be associated with multiple representational contents via different content relations (Chalmers, 2004, p.51).

Secondly, two experiences may have the same local phenomenology but different local content. In such a case, the global phenomenology of each experience may differ according to the background experiences of two subjects. Thirdly, variations in the background situation of each experience may offer different cues to the interpretation of the experience (in a similar way to the colour of a surface being experienced differently in shadow than in bright sunlight). The ambiguity of phenomenal content is not sufficiently acknowledged in formal theories of leadership.

There are many variants of formal leadership theory. They include theories that emphasise leaders' personal traits or styles, and the contingent interaction of leadership style and situation. They also include transactional relationships between leader and followers, the interventionist model of transformational leadership, and the decentralisation of leadership in distributed leadership theory. Leadership theories have waxed and waned in their popularity and some have faded away over time (Bennis, 1959; Leadership: An Overview, 1997). 
Trait theories attempt to link leadership ability to the personal traits of leaders. However, after reviewing over 120 trait studies, Stogdill $(1948,1974)$ concluded that traits alone do not identify leadership ability. He consequently suggested that leadership theory should integrate personal and situational characteristics so as to also take into account the varying demands placed on leaders by different situations. In this view, leadership exists in the relationships between persons in a social situation so that persons who are leaders in one situation may not necessarily be leaders in another (Hersey, 1984).

Lewin et al. (1939) suggest that leaders deal with the situations they find themselves in according to three intrinsic leadership styles: The autocratic style, characterised by the tight control of group activities with decisions made by the leader; the democratic style, characterised by group participation and majority rule; and the laissez-faire style, characterised by very low levels of activity by the leader. A large number of cases failed to reveal a single style of leadership that was universally best across all situations and environments. According to Hersey and Blanchard (1995, p. 148), there is no best style of leadership and leadership effectiveness depends on the combined situational factors of leader, followers and context.

A number of contingency theories have arisen from the attempt to integrate leadership traits or styles to leadership situations. Fiedler $(1967,1972,1978)$ developed a measure of personality, or leadership style, called the "esteem for the least preferred co-worker" or LPC scale and argued that this scale was related to group performance. However, Fiedler's Contingency Model has been widely contested. Whilst contingency theories emphasise the characteristics of leaders and situations, they ignore the characteristics of subordinates or followers.

Transactional leadership theories provide another attempt to account for the interaction of leader and followers. Burns (1978) defined transactional leadership as the initiation of contact with another person to exchange things of value. Importantly, transactional leadership theories have not succeeded in identifying the causes of productive or unproductive leader-follower relationships. Bass (1985) shifted the 
two-way dynamic nature of Burns' transforming leadership to the more interventionist style of transformational leadership. In the transformational leadership model, leaders undertake conscious and deliberate interventions to transform followers and improve the performance of organisations.

Both transactional and transformational leadership models are based on a narrow epistemological framework that is unable to explain the formation of effective administrative practice (Lakomski, 1995). At its core, this epistemological framework is empiricist in nature, utilising questionnaires and operational definitions of theoretical constructs such as, for example, 'leadership'. Such logical empiricist approaches to research are severely restrictive and implausible when applied to highly context dependent social phenomena (Evers and Lakomski, 2000, p. 144).

The ambiguous character of leadership is evident in the countless definitions coined in the literature (Hughes et al., 1995, pp. 41-2), a sample of which is shown below:

- The creative and directive force of morale (Munson, 1921);

- The process by which an agent induces a subordinate to behave in a desired manner (Bennis, 1959, p. 295);

- The presence of a particular influence relationship between two or more persons (Hollander \& Julian, 1969);

- Directing and coordinating the work of group members (Fiedler, 1967);

- An interpersonal relation in which others comply because they want to, not because they have to (Merton, 1969);

- Transforming followers, creating visions of the goals that may be attained and articulating for the followers the ways to attain those goals (Bass, 1985; Tichy and Devanna, 1986);

- The process of influencing an organised group toward accomplishing its goals (Roach \& Behling, 1984); and

- Actions that focus resources to create desirable opportunities (Campbell, 1991).

Leadership resists a general, context free definition and problems of epistemology and methodology have given rise to a large number and diversity of leadership theories (Allix and Gronn, 2005). Methodological choices are theoretically 
constrained, therefore limiting the content and structure of the theories they can support, challenge or produce.

Any methodology will be theoretically constrained because it relies on an underlying theory of knowledge that determines what counts as knowledge and how such knowledge may be acquired. A great deal of educational leadership research is conducted within the hypothetico-deductive framework of logical-empiricist epistemology (Evers and Lakomski, 2000, pp. 65-71). A logical-empiricist epistemology gives undue weight to first and second-hand observation reports and the interpretations of subjects. It has been decisively discredited on philosophical, scientific and educational grounds (see English, 2002; Rizvi, 1991; and Brown, 1977, pp. 81-90). Instead, what is needed is an account of organisational functioning and learning that stands interdependently with a sustainable theory of knowledge - one that deals with questions of truth, reality and the learnability of theories.

\subsection{Distributed leadership theories}

The title, 'Distributed Leadership in Schools' was chosen for this study in 1998 because it reflected my interest in the relationship between the concepts of distributed cognition and leadership, especially in relation to the organisational functioning of schools. At the time this study commenced (in the middle of 1998), the view that leadership could be distributed amongst individuals had made occasional appearances in the literature (Gibb, 1954; Brown and Hosking, 1986; Brown, 1989), but had not attracted strong interest in the research community. In particular, literature searches did not reveal any substantive links made between leadership and distributed cognition theory. Even though the term 'distributed leadership' is now being used increasingly in the literature, few writers have connected it to distributed cognition theory and offered a substantive explanation for such a connection. Instead, the term 'distributed leadership' tends to refer to leadership normatively, in terms of how it ought to be enacted.

The concept of distributed leadership made brief appearances in the literature in the latter half of the $20^{\text {th }}$ Century (Gronn, 2002b, p. 653; Mehar et al., 2006), but did 
not appear in titles until the turn of the $21^{\text {st }}$ century. A comprehensive treatment of distributed leadership is evident in the studies conducted by Spillane et al. (2001) in North America, Gronn (2000a, 2002) in Australia, and Harris (2004, 2005a, 2005b, 2006) in the United Kingdom. According to Gronn (2002a, 2006), distributed leadership is not a new type of leadership, simply a different way to represent leadership in discourse. Here, distributed leadership is interpreted as the enactment of free-will, rather than the exercise of power.

Systematic reviews of distributed leadership (Bennett, et. al, 2003; Woods, et. al, 2004; Leithwood et al., 2006) reveal that the term 'distributed leadership' holds a diversity of meanings, which demonstrate the ambiguity of leadership as a concept. Leithwood et al. (2006, p.6) attempt to identify the nature and causes of leadership, specifically in relation to the question: 'What causes people to attribute leadership status to others and allow themselves to be to be influenced in some fashion?' (Leithwood et al., 2006, p.87). In doing so, they appear to conflate natural causes with the phenomenal perception of influence. In contrast, Woods et al. (2004) suggest that distributed leadership be framed within a form of analytical dualism, based on a distinction between structure and agency. Their epistemology admits structural properties, which exhibit similarities to the social, cultural and artefactual characteristics of distributed cognition theory. It also admits 'agency', which shares some similarities with the phenomenological concept of intentionality and consciousness. They also propose that structure and agency interact but in an indeterminate manner so a single model of distributed leadership becomes unattainable ${ }^{23}$.

Spillane et al. (2007) suggest that leading and managing schools requires the leadership actions of multiple individuals, not simply the actions of those at the top of the organisational hierarchy, or those holding formal leadership designations in their titles or job descriptions. Their analysis of distribution in the work of school principals requires that a distinction be made between leaders and followers within

\footnotetext{
${ }^{23}$ The view of distributed leadership put forward in this thesis shares some similarity with that given by Woods et al. (2004). Both Woods et al. and myself suggest that distributed leadership be framed within some form of dualism.
} 
particular situations. Their study relies on the self-reports of principals, as collected at random intervals in each principal's workday. Hence, even though this approach acknowledges that leadership may be exercised by those who are not formally designated as leaders, the study uses the interpretive observations of principals as its primary source of data.

Some writers describe distributed leadership not as a way of thinking but as a systematic way of organising people within teams and organisations (Barry, 1991; Arrowsmith, 2004; Harris, 2004, 2006; MacBeath, 2005; Ross et al., 2005; Southworth and Doughty, 2006; Leithwood et al., 2007; Mayrowetz et al., 2007). Hence, they frame distributed leadership as an intentional act that may or may not be successful in its implementation. Mayrowetz et al. (2007) also speak of distributed leadership as something that can be 'implemented' as 'distributed leadership projects'. In this view, distributed leadership entails substantial 'work redesign', yet also require the symbolic, positional authority of principals to succeed.

Hatcher (2005) draws links between distributed leadership, self-managed schools and participatory democratic decision-making power. Here, distributed leadership is cast as a means to creating democratic schools. However, Hatcher argues that traditional hierarchical power structures must give way to distributed power if a democratic form of self-management is to occur. In a similar vein, Rosset et al. (2005) cast distributed leadership as a process of influence mediated by 'social and cultural tools'. Their conception of distributed leadership is one that attributes distribution to structural factors so that the degree of distribution begins at zero in the case of individual leadership, and progressively increases as leadership is performed by couples, trios, quads, teams. According to this view, full distribution of leadership occurs in the case of 'collective' leadership. They claim that leadership practice is 'giving way' (Ross et al., 2005, p.131) to distribution, implying that leadership was once concentrated in individuals but is progressing towards collective leadership in contemporary organisations.

To Timperley (2005, p.418), distributed leadership is simply a better model for understanding the realities of schools. Hence, she diverges from the tendency to 
describe distributed leadership normatively as a strategy for school improvement. Wood (2005, p.1105) suggests that distributed leadership is ever-present in leadership practice as a process, so that casting leadership as a concrete object is an error. Attempts to locate leadership in individuals, in the form of personal qualities such as charisma or transformation, only become possible when the abstraction of these qualities is mistaken for concrete reality. Qualities such as these emanate from the realities of specific situations. According to Pfeffer (1977), whilst the realities of situational variables can be described in naturalistic terms, leadership processes cannot:

If the interest is in understanding the causality of social phenomena as reliably and accurately as possible, then the concept of leadership may be a poor place to begin. The issue of the effects of leadership is open to question. But examination of situational variables that accompany more or less leadership effect is a worthwhile task (Pfeffer, 1977, p.111)

Here, Pfeffer distinguishes between leadership 'effects' as subjectively perceived by participants within a specific situation and the causal events assessed by external observers. In this view, leadership may be experienced by social actors as social processes that occur within the constraints of particular situations.

According to Wood (2005), the central epistemological problem of leadership consists in the 'temporary stabilisation of internal movements of difference'. What this amounts to is a naturalistic analysis of the background processes of transformative change, which entail causal-computational processes:

The epistemological problem is not to seek to understand the private world of passions, intentions and influence of individual social actors, or the discrete operation of individuation, but rather to explore the values associated with the internal movement of difference (Wood, 2005, p.1115).

The internal movement of difference is the anchor point of the analysis, to which values may be associated. The essence of leadership - as perceived through the phenomenological perception of difference by social actors - may give rise to a variety of interpretations of experience. Hence, a variety of associations may be 
formed between the stable causal-computational background processes of a situation and the foreground phenomenological perceptions of its participants.

Mehra et al. (2006) argue, from social network analysis, that a greater 'distribution of leadership' across a team is not necessarily related to superior team performance. However, they did find that 'distributed-coordinated' network structures exhibit some association with improved team performance. Importantly, like many other studies their leadership classification scheme relies on the classification of subjects' phenomenological perceptions. Their methodology does not include any justifications that subjects may have given for their decisions about who to nominate or not nominate as leaders. Consequently, the theoretical and cultural influences on those decisions are not revealed. They acknowledge this when they say that access to followers' leadership schemas and the characteristics of leaders (ibid.) might help to explain why different teams adopt different leadership structures. Interestingly, they suggest that leadership networks may be amenable to mathematical analysis via computational modelling. Hence, it appears that they acknowledge the causal-computational nature of organisations whilst also employing the phenomenological perceptions of subjects as a major source of data. They also add that further analysis of subjects' phenomenological experiences may reveal a political dimension to the analysis of distributed leadership.

A review of leadership literature reported by Berson et al. (2006) suggests that leadership is related in some way to organisational learning, though the full range of theories and viewpoints in the literature explains such a relationship in contradictory and inconsistent ways. Another review reported by Porter and McLaughlin (2006) argue that empirical studies of leadership have tended to ignore the dynamic interaction of context and leadership. In contrast, conceptual studies have taken a more dynamic view of the interactions of leadership acts and context. In adopting a naturalistic coherentist epistemology, this study distinguishes between naturalistic and phenomenological views of the world, without affording foundational status to empirical data. 


\subsection{The epistemological claims of this thesis}

What you are about to hear is absolutely true ... only the facts have been changed... ${ }^{24}$

The above statement was made as two comedians reflected on their joint history and relies on the commonly held view that facts are synonymous with truth. Though said in jest, I think it is correct to say that this statement conveys a serious view of knowledge. That is, the theories at the centre of our world-view are more valued than those at the periphery, and are more resistant to revision. The stability of these central theories arises from their coherence with a mutually supporting network of theories (Quine and Ullian, 1978). In contrast, the theories at the periphery of our world view are more tentative and susceptible to revision. These peripheral theories, which include the facts we perceive in different situations, may be discarded or modified without serious implications for our belief system as a whole. This view is at odds with the common, folk-psychological view that considers facts to be the reliable states of affairs from which questionable theories arise. Whilst new theories do arise from our attempts to make sense of the world around us, the facts revealed by observation are theoretically governed. The first epistemological claim of this thesis is that truth claims do not originate from the observations or facts we may summon to support them.

Traditionally, theorising involves the expression of propositions, but here the word 'theory' is used in a broader sense to include that, which is not expressed through language. We perceive only what our cognitive capabilities and theoretical resources enable us to perceive. It is only as we perceive imperfections within those theories or conflicts in their fit with other theories in our world view that we are able to progress to better theories; that is, to learn. Both systematic modes of thought and insight are involved in this learning process.

\footnotetext{
${ }^{24}$ Palin, Michael \& Jones, Terry (2005). Comic Roots. In The Complete Ripping Yarns, London: BBC Worldwide.
} 
Breaking loose from an established point of view requires a discontinuity that cannot be achieved through sequential, logical modes of thought alone. As De Bono (1976, p. 139) has said, an idea which is not right in itself may be used as a steppingstone to an idea which is right. The theories of natural science progress in this way and are striking examples of progress in learning. The second epistemological claim of this thesis is that knowledge (as distinguished from belief) must be learnable. Knowledge arises from learning, and the natural sciences provide our best hope for understanding learning.

The theories we hold impose severe restrictions on perception and learning. Perception arises from the processing of sense data by the theoretical apparatus of human cognition and learning involves the formation or modification of theories. Although language plays a very important part in human cognitive and social processes, it cannot wholly explain the richness of human insight. Knowledge cannot be fully explained as sentence processing, regardless of whether the language in use has an informal structure, as is the case for colloquial language, or a formal structure, as is the case for deductive languages ${ }^{25}$. There is, however, good reason to believe that sensory data are processed according to our cognitive predispositions, or the theories at the core of our worldview. That is, the combined effect of genetic heritage and prior learning serves to filter, classify and organise raw sensory data. The filters of our theoretical world-view are revealed by what we notice in the world; our habitual responses to environmental data reveal the classifications of those data; and our perceptions reveal the theories we use to organise our data. Hence, the third epistemological claim of this thesis is that theories are justified according to their coherence with other theories, including the observation claims of those theories.

Traditionally, knowledge is that which corresponds to a true state of affairs. Now a true state of affairs may be understood as a social constructed reality, or as a metaphysical reality. Determining the nature, boundary and applicability of any socially constructed reality poses severe difficulties given the temporal variance of human knowledge. Conversely, the discovery of previously unknown mathematical

\footnotetext{
${ }^{25}$ The distinction between informal and formal languages is made by Tarski (1983, pp. 152-154).
} 
and scientific truths suggests the existence of a metaphysical reality. The truth value of a theory may be conceptualised in an implicit, deflationary way so that to say 'snow is white' is true just in case snow is white (Stoljar, 1997), or in an explicit, semantic way so that the meaning of a particular explanatory sentence or proposition corresponds to a true state of affairs.

Correspondence cannot be argued justifiably from the supposed certainty of empirical observations. Whilst observations form an important part of learning and theory development, empirical claims inescapably rely on the background theories that determine their scope and significance. All theories gain support through their connection to other theories within a global web of belief, and in the empirical evidence realised by those theories. However, in seeking to explain reality, the best we can ever hope for is approximation to the truth. Knowledge describes the truth content of our theories, whether known explicitly or tacitly. This is the fourth epistemological claim of this thesis.

Finally, and most importantly, to know requires that there be a knower. Understanding how one becomes a knower, or learner, is unlikely to come from the enduring commonsense views of folk psychology. The systematic approaches of the natural sciences provide the most promising means to achieving a better understanding of learning. Since learning is applied to improving our knowledge of organisations, and our knowledge of organisations may be applied to leadership, knowledge of learning is especially relevant to the study of leadership in organisations.

\subsection{Naturalistic Coherentism}

The epistemology adopted in this study is derived from the naturalistic coherentism of Evers and Lakomski (1991, 1995, 1996, 2000) (see also Lakomski, 2005). As its name implies, naturalistic coherentism employs scientific naturalism, an epistemology that makes ready use of our best theories of perception and cognition. It also employs a form of epistemological holism (Evers and Lakomski, 1991, pp. 231-232). Here, I briefly state the case for a naturalistic coherentist 
epistemology as opposed to a foundationalist conception of knowledge. Here, justification, truth and belief are examined in turn.

\subsubsection{Justification}

The implicit beliefs of a theory of leadership should not be accepted without good reason. In epistemology, justification is concerned with demonstrating that a belief is appropriate and reasonable and not simply due to lucky coincidence. Hence, epistemology seeks to distinguish unjustified or false belief from knowledge, which is defined in foundationalism as justified true belief (JTB). According to JTB, foundations have privileged epistemological status over any consequentially justified beliefs. However, the reach of the JTB requirement is severely limited on both an internal and external reading of epistemology. On an internal reading, JTB justification may be shown to be insufficient for knowledge. On an external reading, the justification requirement may be replaced or diminished by recourse to a relational criterion, such as reliability.

On an internal reading, epistemology is concerned with the question of whether rational and empirical knowledge are demarcated or continuous ${ }^{26}$. Extreme rationalism affords foundational status to a priori knowledge, whilst extreme empiricism views a posteriori knowledge as the foundation of knowledge. Hence, extreme foundationalism relies on a sharp distinction between a priori or a posteriori knowledge. A foundationalist view of knowledge typically relies on the justification of a belief statement by inference from one or more epistemically prior belief statements, which are in turn justified by other belief statements at an epistemically lower level, and so on.

There are conspicuous problems with such a chain of justification. From a practical standpoint, humans generally do not operate in such an exhaustive way.

\footnotetext{
${ }^{26}$ Kant's synthetic a priori, which involves experience of some sort, is that which is necessary for human thought. Though tacitly known, the a priori may become rationally accessible through transcendental reflection. Mosser (1999) argues that the both a coherence theory of empirical justification and a correspondence theory of truth are evident in Kant's Critical philosophy, in that it combines moderate empiricism and moderate rationalism within a single epistemology.
} 
Decisions have to be made within the limitations posed by restricted resources of information and time. In practice, foundations may be chosen according to habit, cultural acceptability or the norms of folk-psychology. A radical following of foundationalism, however, requires that a secure foundation of non-inferential knowledge be identified. In the absence of such a foundation, three possible outcomes follow (BonJour, 1999, p. 119):

- A belief statement is eventually reached that cannot be justified in any way thus rendering all the other statements unjustified;

- the chain of justification loops back onto itself in some way so that belief statements become self justifying; or

- the process is deemed acceptable as long as greater significance is assumed for the premise-beliefs that hold up the entire epistemic structure.

In radical foundationalism, a chain of successively justified knowledge claims must begin with self-evident propositions or axioms. According to moderate foundationalism, modest or fallible foundations are assumed for the purposes of the problem at hand. Such foundations are vulnerable to falsification and therefore subject to the possibility of revision or replacement. When that occurs, the entire epistemic structure that the foundations supported falls. An epistemology that employs moderate foundationalism, therefore, is extremely fragile. Ultimately, as Evers and Lakomski (1991, pp. 19-45) explain, certain or incorrigible knowledge cannot be inferred from fallible foundations.

In a strict sense, a foundation does not require justification. It stands apart from reason and is accepted as a matter of conviction. To retain its status as a foundation, a proposition must continue to be self-evident and resist reasonable criticism of any sort. However, the question of how to choose between two conflicting or inconsistent convictions cannot be resolved by such a scheme. Such a choice requires good reason or sufficient evidence or both. In a strict sense, however, foundations stand apart from reason and are self-evident. So if we are going to rely on foundations in a 
theory of knowledge, we hope that they are consistent. The problem of establishing consistency, however, poses its own difficulties ${ }^{27}$.

In foundationalist epistemology, the correctness of any knowledge claim is contingent on both a secure foundation and valid reasoning. It is contingent because the possibility exists that the assumed foundation is not secure or that the reasoning by which it is justified is not valid. If a foundation is necessary, it must contain the reason for itself within itself (Davies, 1992, p. 163). Here the difficulty is that only necessary propositions may follow from necessary propositions; logically they cannot give rise to contingent propositions.

According to A. J. Ayer (1935, p. 31), if a proposition such as the observation statement, 'this is green', is objectively certain or necessary, then it follows that its contradictory, the observation statement, 'this is not green', will be selfcontradictory $^{28}$. However, the statement 'this is not green' is clearly not selfcontradictory. It therefore follows that the statement 'this is green' cannot be necessary; it is contingent on the circumstances to which it refers. Observation statements cannot be necessary; therefore cannot be legitimately accorded foundational status.

The traditional methodology of the sciences is generally understood as being empirical, that is, based on the collection of data in the form of experimental observations and measurements. Francis Bacon proposed in his Novum Organum (1620) the then revolutionary idea that science progresses through a methodical process of inductive reasoning, beginning with sense experience and proceeding to

\footnotetext{
${ }^{27}$ According to Kurt Gödel, 'It is impossible to carry out a proof of consistency merely by reflecting on the concrete combination of symbols and not introducing more abstract elements' (Wang, 1996, p.156). In other words, to prove consistency, one must step outside the object of inquiry.

${ }^{28}$ It must be noted here, however that this argument, put by A. J. Ayer (1935, p. 104), rests on Kant's distinction between analytic propositions, in which the predicate is covertly contained in the subject, and synthetic propositions, in which the predicate does not repeat part or all of the meaning of the subject. Analytic propositions are logically necessary and their denial must be inconsistent or selfcontradictory (See also Bullock, Stallybrass and Trombley, 1988, p. 30). For example, the contradictory of the analytic proposition, 'All bachelors are unmarried men' is, 'All bachelors are married men', which is self-contradictory. Quine criticises the distinction between analytic and synthetic statements, saying that an analytic and its contradiction are 'are the two sides of a single dubious coin' Quine (1951, p.20).
} 
axioms or propositions (Klein, 2004). Hence, if a great many swans are observed in nature and all are found to be white in colour, then by applying the principle of induction it may be concluded that all swans are white. Whilst that conclusion may have seemed perfectly satisfactory in $17^{\text {th }}$ Century Europe, once Europeans encountered black swans in Australia, the proposition, 'all swans are white' was clearly shown to be false (see Popper, 1968). Though many black swans may be observed in Australia, only one need be observed in nature to render the proposition, 'all swans are white', unjustified. Because an infinite number of confirmatory observations are required for the justification of law-like generalisations, induction fails. Fallible foundations lead to fallible generalisations.

The foundationalist epistemology inherent in the classical empiricist tradition relies on foundations in the form of immediate knowledge. In order of decreasing strictness, this immediate knowledge comprises sense data, first person sensory reports, and observation statements. Here, the difficulty is that foundationalist epistemology itself does not qualify as immediate knowledge. It simply does not take the form of sense data, or first person sensory reports, or observation statements and therefore does not qualify as foundational knowledge by its own account. Foundationalism is therefore incoherent.

Attempts to give operational definitions to unobservables, such as 'leadership' or 'organisation' depend on a sharp distinction between theoretical and observational terms. However, the interdependence of theory and evidence occurs through the theoretical predispositions of unmediated observations, the use of theory to interpret readings or perceptions, and the use of theoretically structured instruments or apparatus (Evers and Lakomski, 1991, p. 34). In this study of leadership in schools, foundationalist justification is rejected. Instead, theories of school organisation and leadership are evaluated according to a naturalistic coherence epistemology, which does not sharply distinguish between theoretical and observation terms. What is important here is the coherence of organisation theories with our most significant global theories including scientific accounts of human learning. 
Naturalistic coherentism avoids the foundational status afforded to operational definitions and propositional logic by logical positivism, or the value-free observation claims of logical empiricism. It blurs the distinction between observation and theory, fact and value. Our best scientific accounts of nature posit unobservable concepts in the same way that organisation theories do. In both the natural sciences and organisation theory, various observational instruments are employed to enable otherwise unobservable entities to be observed. These instruments are theoretically constructed and the observations taken with them unavoidably depend on the suitability and reliability of those theories for the tasks at hand (Boyd, 2002). This is the case when, for example, an electronic data logging instrument is being used to measure the end-point of an acid-base titration in a laboratory, or a survey instrument is being used to measure the state of staff health and well-being in a school. In both cases, the measurement instruments are constructed according to pre-existing theories. Thus, the distinction between science and organisation theory is blurred.

All theories, including scientific theories, organisation theories, and the theories used to construct observational instruments, require evaluation. Because observational evidence is itself theory-dependent, it cannot fulfil this requirement. Therefore, a naturalistic coherentist epistemology evaluates theories according to a common set of superempirical virtues (Evers and Lakomski, 1991, p. 231). These virtues include conservatism, simplicity, consistency, comprehensiveness, fecundity, explanatory unity, refutability, and learnability (Allix, 2003). All of these virtues are traditional and accepted as requirements for good theory-making. Through reliance on superempirical virtues, the naturalistic coherentism of Evers and Lakomski (1991) employs a coherence theory of evidence or justification ${ }^{29}$. Here, the theory under investigation is evaluated in relation to other trusted theories. Through a process of feedback and learning, adjustments are made to the theory so that the match between the theory and reality, that is the truth value of a theory, progressively improves.

\footnotetext{
${ }^{29}$ Evers and Lakomski (1991) distinguish between a coherence theory of evidence and a coherence theory of truth. The latter relies on truth propositions which cannot be specified without contradicting the coherence requirement (see also Young, 2001, June 21).
} 


\subsubsection{Truth}

We construct theories to explain leadership and organisational functioning because we desire the truth about these matters. The quest for knowledge is necessarily the search for true knowledge, free from error or falsehood. When Pontius Pilate said, "What is truth?" he implied that truth was unknowable. This is the position of extreme scepticism, which denies any claims to knowledge. However, extreme scepticism is both subjectively and objectively untenable. To make the subjective statement, "I know that I do not know" is paradoxical and logically unresolvable. A similar problem occurs with the objective statements, A and B, below $^{30}$ :

Consider the statement: "There is at least one true proposition." Call this proposition A. Is A necessarily true? Suppose I contend that A is false. Call this proposition B: "A is false." But if A is false, so is B, because B is a proposition, and if $\mathrm{A}$ is false there are no true propositions. So A must be true. It is therefore logically impossible for there to exist no true propositions (Davies, 1992, pp. 177-178).

To accept that I know that I do not know or that there are no true propositions it would seem that I must abandon reason. It is reasonable for me to accept, firstly, that I know some things and, secondly, that true propositions exist ${ }^{31}$.

Knowledge is that which we believe to be a true state of affairs. What is it that makes any knowledge claim true? Various attempts have been made to securely determine what it is that distinguishes truth from falsehood, though most rely on some sort of formal scheme of argument. Whilst such schemes succeed in broadly elucidating the positions in question, none has succeeded in rendering those positions completely secure. It seems that all logical arguments run into difficulties at some point. Knockdown arguments are hard to come by in epistemology simply because

\footnotetext{
${ }^{30}$ The universal problems posed by antinomies such as this are expressed in Russell's Paradox (Irvine, June 2004).

${ }^{31}$ However, such propositions must in themselves be necessary and the relationship between necessary and contingent propositions poses deep philosophical problems, which cannot be satisfactorily dealt with here.
} 
no epistemological system can be completely divorced from assumption, interpretation of meaning, paradox and uncertainty.

If we accept that uncertainty pervades truth, perhaps all we can hope for is consistency and harmony in a system of beliefs. This is the holistic view explained within the coherence theory of truth (not to be confused with the coherence theory of evidence). In this theory, coherence is applied epistemologically as the determinant of truth. However, the coherence theory of truth does not provide a satisfactory means for choosing between two internally coherent but externally contradictory belief systems. Neither can it satisfactorily deal with the possibility of true propositions that do not cohere with any other beliefs (Young, 2001). Whilst incoherencies or inconsistencies are reliable indicators of falsity in a theory, coherence or consistency cannot determine the truth of a proposition (Popper, 1972, p. 226). Whilst certain facts may have objective reality, there is a difficulty in knowing when we have found them. Cherished theories or facts may at some time in the future, be shown to be incoherent or false and therefore require revision or abandonment. The search for knowledge is the search for true theories. However, if we adopt a coherence theory of truth we are faced with the possibility that we may never find them, nor recognise them if we do.

Popper (1972) criticises the coherence theory of truth for mistaking consistency for truth, the evidence theory of truth for mistaking attributions of truth for truth itself, and the pragmatic theory of truth for mistaking usefulness for truth. Neither consistency nor evidence guarantee truth, and a considerable delay, even centuries, may ensue before a discovery is usefully applied in any field. Each of these theories, in Popper's (1972, p. 225) view, are subjective in the sense that they view knowledge only as a special kind of mental state or disposition. He contrasts them with the objective (or metalogical) theory of truth of Alfred Tarski, in which truth is defined as correspondence with the facts.

Tarski's 1929 analysis of the concept of truth in formalised languages (1983, pp. 152-278), set out to 
construct - with reference to a given language - a materially adequate and formally correct definition of the term "true sentence" (Tarski, 1983, p. 152).

Beginning with Aristotle's conception of truth, Tarski firstly showed that in colloquial language it is not possible to define truth or even to use the notion of truth in a logically consistent manner. With respect to formalised languages, he concludes that a satisfactory definition of truth is possible, but only by including the concept of truth in the primitive concepts of a higher order metalanguage ${ }^{32}$.

It seems that any attempt to base epistemological conclusions on a foundational theory of truth must fail (Horwich, 1994, p. xvii). Tarski demonstrated the indeterminacy of truth in colloquial languages. This result places severe restrictions on truth as a concept, in that the satisfactory definition of truth in formal languages is subject to the problem of incompleteness ${ }^{33}$. The verification of truth may be doubted based on the methods of verification, and if truth is a primitive, inexplicable property then we can have no reason for assuming that our propositions are true. Finally, the demonstration of truth, ' $T$ ', in the form, ' $T$ is true', based on some epistemological or metaphysical characteristic leads to a circular argument. That is, the demonstration that ' $T$ ' is equivalent to ' $T$ is true' relies on an equivalence schema, the truth of which cannot be determined without reference to itself.

One solution to these problems is to adopt the deflationary theory of truth (Williams, 1986; Stoljar, 1997), which would seem to remove the need for metaphysics, the puzzle of the nature of truth, and the need to deal with semantic terms. However, in the absence of a theory of meaning and a means for satisfying the correspondence intuition, there is not sufficient reason for choosing the deflationary

\footnotetext{
${ }^{32}$ Difficulties in establishing the semantics of a formalised language led to Tarski (1983, p.270) revising his earlier conclusions to remove reference to the semantic categories of formalised languages. However, attempts to completely remove semantics from accounts of truth have also run into problems (see Field, 1994, pp.285-314).

${ }^{33}$ Tarski (1983, p.273) explains that completeness can only be achieved if the employed metalanguage is richer than the object language it describes. That is, the metalanguage must be of a higher order than the object language. This is not possible for object languages of infinite order and formal languages of finite order suffer from an absence of semantic terms rendering them vulnerable to extravagant claims (See Tarski, 1994, p.113 and Field, 1994).
} 
theory of truth over correspondence theory. The deflationary theory of truth also appears to be identical with its complete opposite, inflationism.

In their earlier representations of naturalistic coherentism, Evers and Lakomski paired coherence justification with a correspondence theory of truth:

\begin{abstract}
For us, a true theory is one which corresponds to, or matches up with, the way the world is. How we know what the world is like, what objects exist, is something we rely on our best theory to tell us. Once the theory of evidence has done its work, what exists is given by the posits of our best theory, with the details of correspondence relations between theory and world also being determined by that same theory (Evers and Lakomski, 1991, p. 42).
\end{abstract}

In naturalistic coherentism any theory forming a part of a global world view, including a theory of truth, is subject to coherence justification and therefore liable to revision.

Ultimately, the superempirical values of naturalistic coherentism will guide the choice between deflationary theory and correspondence theory of truth. In applying the superempirical virtues to the evaluation of organisation theories, a naturalistic coherentist epistemology chooses theories that exhibit greater internal consistencies and therefore fewer anomalies or counter-examples, simpler and more comprehensive explanations and predictions, and fewer theoretical claims made without evidence. Such theories are connected to or comparable with other trusted theories and experiences and are therefore learnable (Evers and Lakomski, 1991, p. 9). It is in this way that beliefs may be justified.

\title{
2.4.3 Belief
}

The concept of leadership entails beliefs about its identity, properties or meaning. Beliefs are necessary if ignorance is to be avoided. The Nicene Creed, compiled in A.D. 381, and beginning with the words, 'I believe...', presents a set of commonly held Christian doctrines. Though derived from earlier texts, it does not attempt to justify the statements it contains. Its purpose was simply to distinguish one particular set of beliefs from another. The purpose of epistemology, however, is to seek a 
rational means for evaluating competing beliefs. This purpose may be inferred from the roots of the word 'epistemology', as found in the Ancient Greek words: epistemé

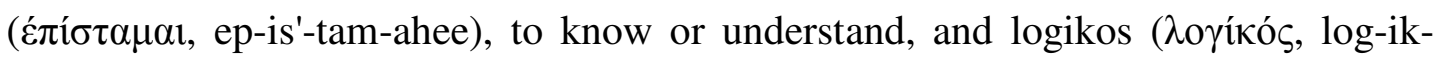
os'), to give reasons.

Are beliefs purely the outcome of human experience? According to Kant (1964), beliefs are only possible because 'the epistemic subject contributes a set of universal and necessary conditions for the possibility of experience' (Mosser, 1999, p. 44). In other words, in the absence of some sort of conceptual apparatus for the processing of theories or observations, perception is not possible and world experience becomes inaccessible. This is evident in Kant's view of sensibility as an essential but passive component of human experience. In this, the subject contributes special and temporal intuition (though this is not equivalent to the construction of experience or the creation of content). Beliefs do not arise from human experience alone but in accordance with the bounds of human intuition and reason. That is, a minimal set of a priori conditions for possible experience are also the necessary conditions for belief. These conditions are not truth conditions in themselves, but concern the universals that allow a given proposition to be judged true or false. Consequently, that which we know a priori concerning such judgements is distinct from the truth or falsity of a given proposition. The a priori then are the necessary conditions of belief whilst the relations between beliefs, including existing beliefs and cognitively spontaneous beliefs, is the main concern of coherence justification (BonJour, 1985).

From a phenomenological standpoint, epistemology comprises a continuous stream of self-awareness so that truth is grasped in consciousness. In phenomenology, therefore, a priori knowledge is identified with the intuition of pure essences, which stand independently of actual perceptions (Levinas, 1973, pp. 107, 141). Hence, truth on a phenomenological reading does not belong essentially to judgments but to intuitive intentionality (Levinas, 1973, pp. 133, 150). In contrast to naturalism, phenomenological truth is grasped from within consciousness; that is, from a first-person standpoint (Hutcheson, 1981). Phenomenology also claims an intersubjective intuition of the life of others and of reality. This might entail the 
grasping of the existence of others as analogies of self; however, it is not clear what this intersubjective intuition constitutes.

From a naturalistic standpoint, the a priori is inherent in the human cognitive apparatus, but not of necessity, in the form of rules as Kant originally proposed. From what is currently known about the science of human cognition, the underlying structure of human understanding seems more likely to be determined by emerging patterns than by directive rules. This is the nature of parallel-distributed processing systems, which arose from processes of feedback, symbiosis and evolution.

Beliefs, like information, may be subject to randomness in the sense that some data contain no underlying patterns (Shannon, 1948). Concerning mathematics, Chaitin (1996) has shown that there exist questions that cannot be resolved by mathematical reasoning, simply because there is no pattern or law to be discovered. Similarly, in nature we find uncertainty in the form of non-linear dynamics and quantum uncertainty. Chaitin assures us that this does not mean that mathematics, or the universe, are lawless, just that we need to apply statistical or stochastic laws to their analysis instead of deterministic ones. However, statistical laws require empirical evidence for their construction and are therefore subject to the problems of induction and the theory-dependence of observation mentioned previously. We are again faced with the limits of reason in that we may believe something to be true, but without good reason:

Most people believe that anything that is true is true for a reason. These theorems show that some things are true for no reason at all, i.e., accidentally, or at random (Chaitin, 1996, p. 270)

What can we say about knowledge when beliefs are true for no reason at all? This may be said to be the case for propositional knowledge in the case of Gettier examples (Zagzebski, 1999, p. 100). Gettier examples are cases when true beliefs cannot qualify as knowledge because they are true because of chance alone. If knowledge is associated with notions of virtue in truth, then in Gettier examples virtue does not apply and the conditions for knowledge are not satisfied. In the words of Aristotle, 'To leave the greatest and noblest of things to chance would hardly be 
right' (Nicomachean Ethics 1109b25, cited in Zagzebski 1999 p. 100). Intellectual virtues are required for knowledge. The 'super-empirical' criteria of theoretical simplicity, consistency, comprehensiveness, conservativeness and fecundity (Evers and Lakomski, 1996, p. 15) are such virtues.

Chance applies not only to the intellect, but also to practical wisdom. In traditional Anglo-Australian culture, a particularly sensational sporting achievement may be referred to ironically as a 'fluke'; that is, as a chance event that is not likely to be repeated. Here, the term 'fluke' refers to an achievement that is due not to the knowledge or skill of the player, but to chance or coincidence. If human achievements may be attributed to chance alone, then any claim to knowledge or skill because of those achievements is without virtue.

Applying the idea of intellectual virtue to schools, consider the hypothetical proposition that the unusually high academic performance of a teacher's students were due to her superior competence as a teacher. If the claim to superior professional ability is to stand, the possibility of a Gettier example, according to which the high academic results occur by chance, must be ruled out. The attributed causality of the teacher's individual professional ability would be undermined if certain factors outside the teacher's control were significant determinants of the students' academic successes. Such factors could include:

- strong social bonds between students that improve their ability to learn;

- a sequence of classes, as organised in the school timetable, that favour the productive learning of that particular group of students;

- circumstances in the students' home lives that facilitate the students' preparedness for learning;

- extraordinary curriculum and teaching support, which reduces the teacher's workload and cognitive burdens so that a high standard of teacher preparation becomes possible; or

- a particularly supportive collegiate climate amongst the teaching staff, which lifts the teacher's mood and teaching ability above what it would otherwise be. 
None of the cases described in these examples need to arise from actions, specifically designed to support the learning of that particular group of students. Neither, can they be determined by the teacher. In such a hypothetical case, the true causes of improved student learning may be due to the events that led to the particular classroom situation, not necessarily to the knowledge or skills of the teacher ${ }^{34}$.

In a case of this sort, an observer might be justified in believing the individual teacher, apart from all other factors, had demonstrated superior professional ability. However, whilst that conclusion may count as Justified True Belief, its status as knowledge may be questioned (Williams, 1999, pp. 5-6). If situational factors (that is the chance coincidence of events) turn out to be the most significant causes of the students' high academic results, then this example would constitute a Gettier case. However, if foundations are not relied upon and the holistic, distributed nature of knowledge is acknowledged, then a less restricted and more intuitively acceptable view of knowledge becomes possible.

Belief is made possible by a priori conditions, which include our deeper cognitive capabilities as well as the universal conditions of the world (Kant, 1964). Through the social distribution of knowledge, we come to treat certain beliefs as if they had arisen a priori. There may also exist at the centre of our web of belief, beliefs that are strongly held, but without good reason. In these cases, it is not helpful to deny their existence, though it must be said that we may hold these kinds of theories without being consciously aware of their presence, or their influence on our world-view. That is, their origins may be a priori. Though they resist justification when viewed in isolation, theories like these (when their existence and influence have been acknowledged) may be evaluated according to their relations to reasonably justified beliefs. The super-empirical virtues of naturalistic coherentism may put the justification of such beliefs within reach.

\footnotetext{
${ }^{34}$ Unless the word 'cause' is used here in the sense of Aristotle's concept of material cause (see Chapter 6, section 6.1.2), which in any case cannot be brought about by the teacher.
} 


\subsection{Limitations to knowledge}

The knowledge claims of educational researchers are attempts to demarcate true beliefs from those that are false. Such claims may be made in both naturalistic and phenomenological terms. According to Quine (1995, pp. 15-16), naturalism and mathematical logic are the physicalist alternatives to phenomenalism, which he claims conceptualises material things in terms of sense data. In this view, naturalism employs a normative epistemology that does not rely on any general calibration or comparative scale. Rather, the normative values of conservatism and simplicity are employed aesthetically as part of the 'art of science' (Quine, 1995, p. 49). Conservatism requires that a theory not conflict with any existing well-tested and accepted theories. The virtue of simplicity requires the application of Ockham's razor, by which the theory relying on fewer extraneous or untested assumptions is preferred over another.

The values of conservatism and simplicity may be accompanied by consistency, comprehensiveness, fecundity, explanatory unity, refutability, and learnability. The criterion of consistency favours the theory that exhibits fewer internal anomalies, whilst a more comprehensive theory exhibits greater scope. Fecundity or fruitfulness is a pragmatic value that assesses the relative usefulness of a theory in terms of its predicted and existing applications. Explanatory unity describes a theory's logical accord with other theories, whilst a theory that makes riskier claims and is therefore most vulnerable to falsification is at risk of refutability. Finally, learnability refers to the methodological coherence of a theory (Kelly, 2001, p. 17).

Quine's holism makes free use of whatever scientific findings are needed to fulfil the purpose of normative epistemology (Quine, 1986, p. 664-5), and as Kornblith (1999, p. 167) has said, 'we must allow the inquiry itself to dictate what is relevant as our investigation unfolds'. Because such information may include 'environmentinvolving perception' and/or 'neighbour-involving testimony', any divide between internal and external sources of knowledge is put into question (Sosa, 1999, p. 156). Whilst a naturalistic epistemology, which is informed by empirical evidence, appears to avoid such a divide by taking a continuous view of knowledge, the problem of 
incompleteness cannot be avoided. In research into the leadership and organisational functioning of schools there are no hard and fast rules for distinguishing between internal or external sources of information, or between local or global analyses.

\subsubsection{The limitations of naturalistic coherentism}

A naturalistic analysis of schools, framed in terms of cause and effect, is subject to limitations that also apply to the natural sciences. The differentiation of entities or properties such as local or global, fact or value, and mind or body, have arisen in our attempts to classify the information available to us. Indeed, it is difficult to believe that learning can proceed without any form of differentiation or classification occurring. How are such distinctions to be acknowledged whilst also embracing a seamless and continuous view of knowledge? One answer is to acknowledge that dissolving a duality by excluding one of its entities (or perspectives) leads to incompleteness - one entity cannot replace the other (Hildreth and Kimble, 2002). Another is to acknowledge the interdependence of one on the other.

Take the example of quantum duality. An electron is widely acknowledged to exhibit the properties of both particles and waves. Quantum mechanics, which is strongly supported by evidence, has resolved this paradox, but not without considerable debate about its philosophical implications (Laudisa and Rovelli, 2005). According to quantum mechanics, an electron moves about in an undetermined and random manner that cannot be fully explained in terms of cause and effect. The trajectories and positions of an electron cannot be pinned down because an electron simply does not possess a precise momentum at a particular instant of time. Though collections of electrons are constrained by their general context as described by a probability function, the movements of individual electrons are events 'on their own' - events that do not interlock with, or depend on, other events.

C. S. Lewis (1947) uses the example of quantum theory to support his argument that naturalistic explanations, whether in practice or in principle, cannot possibly be the 'whole show'. He calls the chance events of sub-atomic particles sub-natural events, because they fall outside the deterministic web of nature and cannot be fully 
explained in terms of cause and effect. Similar examples may be found in mathematics and philosophy. The theorems of Gödel, Church, Turing and Chaitin, point to the existence of randomness in mathematics and information theory. Similarly, Kant's a priori, posits the universal, or necessary conditions for knowledge, which are unknowable. These things cannot be explained in causal terms, yet like quantum uncertainty, they appear to feed into, or influence, nature.

If there is a sub-nature that feeds into nature, as Lewis suggests, could there exist a super-nature that feeds into nature? Lewis suggests that our ability to think and reason is part of such a super-nature in the same way that knowledge of a machine is not the same as the machine itself. However, any super-nature will be inaccessible to rational or empirical inquiry in the same way that sub-nature is inaccessible to inquiry. Nature, however, is accessible to inquiry and it is within the natural sciences that we can expect to carry out our most fruitful investigations.

Of course, if nature is all there is, it makes no sense to look for knowledge anywhere other than in nature. Naturalism is an essential part of a naturalistic coherentist epistemology, but it cannot be the whole story in the same way that empirical knowledge is not the whole story about knowledge. Many scientific advances have occurred through a systematic approach to theory and observation. However, extraordinary chance events, imaginative leaps and insights have also been important to scientific progress. These events are not amenable to normal causal explanations, yet to deny their occurrence is as defeasible as their justification.

The experiences of Henri Poincaré, Roger Penrose and Fred Hoyle, as referred to by Davies (1992, pp. 145-146, 228-229), are cases where the individuals concerned reported a flash of inspiration, together with feelings of elation, in relation to problems they had been tackling. It was only later that the correctness of those sudden and unexpectedly received solutions were rigorously demonstrated. How is it that mathematical truths, in pure or applied mathematics or physics, appear to be discovered rather than created? If they exist before discovery, they must exist somewhere. In a purely naturalist epistemology, there is no place for such things. There simply is no closed and logical scheme of thought or observation that can 
provide a complete and self-consistent explanation for everything (Davies, 1992, p. 226).

A strict naturalism cannot deal with moral judgements or values without treating them as the inevitable outcome of deterministic natural processes. If we are to make moral judgments, we must believe that human conscience is not a deterministic product of nature. In addition, if we are in fact free to choose certain values over others, and therefore free to act in whatever way we choose, then we must possess free-will, something C.S. Lewis denies is open to pure naturalism:

\begin{abstract}
No thoroughgoing Naturalist believes in free-will: for free-will would mean that human beings have the power of independent action, the power of doing something more or other than what was involved by the total series of events. And any such separate power of originating events is what the Naturalist denies. Spontaneity, originality, action, 'on its own', is a privilege reserved for 'the whole show', which he calls Nature (Lewis, 1960, p. 11).
\end{abstract}

The dualist nature of quantum theory and the limits to deterministic explanations posed by random events are not conducive to radical coherentism or radical naturalism. I have chosen to adopt a moderate coherentism together with a moderate naturalism, to take a position somewhere between a purely dualist and purely holistic epistemology. That requires that both rational and empirical evidence be admitted to an analysis of school leadership and functioning, whilst also acknowledging the resilience of a priori theoretical world-views and the theory-dependence of $a$ posteriori observations.

\title{
2.5.2 The limitations of empirical evidence
}

Educational research may draw on empirical evidence but it is important to acknowledge that the background theories of subjects and researchers will affect the way raw data is selected, presented and comprehended. Whilst empirical evidence and theory should form a coherent whole, empirical evidence itself is theorydependent. This is the case for both the natural and social sciences and has important implications for the progressive improvement of theories of leadership and organisational functioning in schools. 
According to Popper (1972, p. vii), knowledge, particularly scientific knowledge, progresses in increments by guesses, anticipations, tentative solutions, or conjectures, which are in turn restrained by criticism or refutations. He argues that a conjecture may survive severely critical tests, but cannot be established as being certainly true or even as being more probable than some other conjecture. Since we cannot be positively certain of the truth of any knowledge claim, it is not possible for any knowledge claim to have authority over another. In this view, authoritarian claims to knowledge such as being 'the best', 'the wisest', 'the expert', 'all powerful', 'the people', or 'the majority' are without epistemological justification. Of course, we may choose one theory over another simply because we judge it to be better, wiser, more powerful, or more popular. However, this would simply be for the reason that it is better to make a commitment of some sort rather than no commitment at all. Such commitments can be given up each time a better, more fruitful theory is found.

Any theory will comprise a set of interdependent rational and empirical claims. However, one important requirement for a scientific theory is that its theoretical claims can be rigorously tested. Empirical data comprises the evidence we perceive through our senses. In its pure form, empirical data is only available to first-person observers. Second-hand reports of observations are not purely empirical as they are affected by the ambiguities of language and form that stem from their inescapably theoretical nature.

Empirical evidence may be used to challenge popular theories. In September 2003, the journal Nature (see Frumkin, Shimron, and Rosenbaum, 2003) published news that the Old Testament structure, the Tunnel of Siloam, was the first such structure to be accurately dated using a radiocarbon dating method. The importance of this calculated date, as reported in the Melbourne newspaper The Age (Cauchi, 2003a), was that it refuted theories that this tunnel was not the tunnel of Siloam as mentioned in the Old Testament books of Kings and Chronicles. Similarly, in the October 2004 issue of Nature (Lahr and Foley, 2004), the discovery of a human-like skeleton in a limestone cave on the island of Flores, west of Timor was reported. According to media sources (see Cauchi, 2004; Pountney, 2004), this discovery 
challenged the existing human family tree and the conventional relationship between brain size and intelligence. In examples such as these, empirical data are cited as refutations of particular theoretical claims.

The interpretation of empirical data is a theoretically dependent exercise. Reported observations may be cited as confirmations of a theory, or as refutations, or as inconclusive findings that may be safely ignored. The reliability or significance of observations may be questioned according to their predictability, applicability, accuracy or validity. However, when observations challenge a pet theory, there is a greater incentive to question their validity.

One theory, that has sadly gained a popular following, says the American moon landings never happened. This conspiracy theory's popularity was encouraged by the screening of a television documentary that purports to show evidence that the moon landings did not take place $\mathrm{e}^{35}$. The popularity of this hoax is significant enough for a number of websites (including one maintained by NASA) ${ }^{36}$ to host arguments explaining the fallacious claims of this conspiracy theory. Media sources report that scientists are proposing to obtain direct observational evidence of the moon landings by using a powerful telescope (see Matthews, 2002). The interesting thing to note here is that in the case of some items of empirical evidence, the same evidence may be used to support and refute the argument of the conspiracy theorists.

Ultimately, no empirical data collected by observation, measurement or experiment can be accepted at 'face value'. All observations are inescapably evaluated and interpreted in relation to their background theories. Quantitative data will require correction in one way or another: parallax errors, pressure dependencies, sampling errors, and bias or systematic errors all undermine the integrity of data if not accounted for or corrected in some way. Once corrected, numerical data may be processed statistically to yield aggregate data such as mean and standard variation,

\footnotetext{
${ }^{35}$ I recall this documentary being screened one year on April $1^{\text {st }}$ in Melbourne (what an appropriate choice of day!). The next day it became a popular topic of discussion in my science classes, the discussion always being initiated by students.

${ }^{36}$ See http://www.apollo-hoax.me.uk/index.html, http://science.nasa.gov/headlines/y2001/ast23feb_2.htm
} 
together with random errors or scatter plots. Whilst this may simplify the data analysis, reliance on aggregate data may also remove some important particularities. Similarly, qualitative data must be interpreted. Qualitative data may be subject to bias due to the theories used by the researcher so that attention is paid to some features of the data whilst other features are ignored. Alternatively, multiple interpretations of the data may be equally feasible so that the choosing one interpretation over another becomes an arbitrary choice. The quantitative and qualitative methods used to process empirical data, are themselves, theoretically governed.

Ultimately, choices must be made about which data will be included in an analysis and the significance attributed to particular sub-sets of data. In the process, some data may be eliminated because they are outliers or because it is decided that they are not relevant to the particular question under investigation. The examples in Australian Law of the two separate cases of Azaria Chamberlain and Jaidyn Leskie highlight the potential for ambiguity in the interpretation of forensic data (Russell, undated; Cauchi 2003b). Some data may be discarded because they flagrantly contradict trusted theories and are therefore not trusted (Bunge, 1983, p. 67-8). Likewise, data may be retained because they appear to confirm a trusted theory. The interdependence of theory and data, and of theory and practice, arises within a selfsupporting web of knowledge.

It is important to acknowledge the interdependence of theory and data when studying the functioning and leadership of schools. Empirical data alone cannot challenge the weaknesses of theories at the centre of our world view. The pervasive incoherence of leadership theories and their inability to explain organisational functioning may be due in part to their reliance on logical-empiricist epistemologies. Epistemologies that rely explicitly or implicitly on logical empiricism give undue status to observation over theory, particularly in relation to survey and interview data. 


\subsubsection{The limitations of theories}

Many beliefs about leadership persist, simply because the theories at the centre of our web of knowledge are resistant to revision. So resilient are some theories that empirical evidence may be found for things later found to be nonexistent. One dominant example is the theory of the Ether, which was connected to classical Newtonian ideas of absolute space and absolute time (Galison, 2003, p. 236). The Ether, which had origins in the philosophy of Aristotle, was described in the late nineteenth century as

A hypothetical medium of extreme tenuity and elasticity supposed to be diffused throughout all space (as well as among the molecules of which solid bodies are composed), and to be the medium for the transmission of light and heat (Whitney, 1899, p. 2016).

As early as 1895 , Einstein was troubled by the idea of radiation as a wave in static, material Ether. He considered how a swell would occur in the Ether as a wave passed through it. Suppose, he later recalled,

One could catch up with a light wave and ride it, so to speak, as classical physics might imply. Then one would see the electromagnetic wave unfold before him, the field undulating in space but frozen in time. But nothing like such a frozen wave had ever been observed (Galison, 2003, p. 228).

Einstein's critical attitude towards the Ether as a form of absolute space was encouraged by the writings of others. David Hume's (1739) Treatise on Human Nature, John Stuart Mill's (1843) System of Logic, Ernest Mach's (1883) criticism of absolute time in The Science of Mechanics, Karl Pearson's (1892) The Grammar of Science, Richard Avenarius' (1888) Critique of Pure Experience, and Poincaré's (1905) Science and Hypothesis all influenced Einstein's thinking. It seems that, the writings of other thinkers and the social context of the day played an important role in the development of Einstein's thinking. Through his work in the Bern Patent Office from 1902, Einstein became preoccupied with practical applications of electromagnetism and the synchronisation of time, which had become very important to the clock industry, the military and transportation systems in Europe and North America. 
Einstein was not simply concerned with a lack of empirical data in support of the theory of the Ether, but that the Ether lacked physical meaning (Galison, 2003, p. 231). Clerk Maxwell purportedly calculated the density of the Ether (reported by A. Daniel in Whitney, 1899 p. 2016) as $9.36 \times 10^{-19}$ times the density of water. Despite this apparent empirical evidence in support of its existence, the Ether was found to add unnecessary theoretical complexity to the explanation of electromagnetism. Consequently, theories of material Ether, as a substance through which electromagnetic radiation must pass, were abandoned in favour of a non-material vacuum.

The abandonment of the ether, and the related concepts of absolute space and absolute time, paved the way for the Special and General Theories of Relativity. These theories now have important practical applications in technologies such as, for example, the Global Positioning Satellite (GPS) system. This system, which is used for military and civilian navigation purposes, could not function if it were constructed according to classical physics and the theory of the Ether. Hence, the removal of the Ether from our web of knowledge has strengthened rather than weakened our knowledge of the world around us. The supposed empirical evidence for the density of the Ether did not eliminate the theoretical difficulties that the Ether presented. What mattered was the relationship of the Ether theory to other theories, particularly the wave theory of light and electromagnetic theory.

Similarly, rather than seeking empirical confirmations or disconfirmations for theories of school leadership and organisational functioning, we should be seeking theoretical coherence. What are needed are theories of school functioning that cohere with our most reliable theories, those that exhibit the superempirical virtues of conservatism, simplicity, consistency, comprehensiveness, fecundity, explanatory unity, refutability, and learnability.

\subsection{Creating better theories}

Educational researchers seek better theories. For Kuhn, theory improvement occurs through shifts of theoretical perspective or paradigm: 
The man who embraces a new paradigm at an early stage must often do so in defiance of the evidence provided by problem-solving. He must, that is, have faith that the new paradigm will succeed with the many large problems that confront it, knowing that the older paradigm has failed with few. A decision of that kind can only be made on faith (Kuhn, 1970, p. 158).

Kant also considered the relationship between faith and reason. He distinguished theoretical reason, constrained by a spatio-temporal causal framework, from practical reason, the exercise of freedom. He argued that if these two types of reason are not distinguished, an antinomy of practical reason follows and theoretical reason oversteps its limits. The moral imperative of practical reason, the highest good, cannot be met by theoretical reason. The rightness of actions cannot be explained by causal processes. The antinomy occurs in expecting the moral actions of practical reason to produce that which they do not have the power to produce:

The practical connexion of causes and effects in the world, as the result of the determination of the will, does not depend upon the moral dispositions of the will, but on the knowledge of the laws of nature and the physical power to use them for one's purposes (Kant, 2005, p. 73).

Theoretical reason arises from the question, 'What can I know?' and practical reason from the question, 'What ought I do?' However, it is only in the question, 'For what may I hope?', that Kant thinks theoretical reason and practical reason may eventually converge (Rossi, 2005). That would require that the metaphysical virtues of hope, purpose and faith stand alongside the physical nature of space, time and matter. In schools, the question, 'What can I know?' relates principally to the physical reality of schools and the processes of knowledge and learning. In contrast, the question, 'What ought I do?' concerns human intention and decision-making. Both of these questions are theoretical questions, with the first being consistent with a naturalistic perspective and the other phenomenological. However, the question, 'For what may I hope?' concerns the purposes and outcomes of practical human action in the physical world. A theory of school leadership that entails practical wisdom will be one that acknowledges both the phenomenological world of human intentions and the world of cause and effect, as understood naturalistically. 


\subsubsection{The construction and evaluation of theories}

An educational leadership theory that entails practical wisdom will be one that acknowledges the reality of the physical world, as portrayed by the theories of natural science, and the perceived world of human plans, hopes and beliefs. According to Aristotle, $(1988,1995,2000)$ in the Nicomachean Ethics, reason arises from the combination of practical wisdom (or phronesis) and wisdom, and wisdom arises from both intellectual and scientific knowledge.

Popper (1972, p. 197) classifies intellectual knowledge within three broad groups with the first group comprising logical and mathematical theories, the second group comprising empirical and scientific theories, and the third group comprising philosophical and metaphysical theories. He asserts that logical and mathematical theories must be testable, first superficially, then more severely. If the refutation is unsuccessful, then an attempt is made to refute its negation. If severe tests of the negation do not succeed in refuting the theory, then in mathematics it may be possible to arrive at a proof. Unless this is seen as a purely evolutionary, physical process governed by a combination of physical laws and chance, a test requires a theory to be tested in accordance with conscious human will.

Proof, Popper argues, is not attainable for scientific theories but they may be vulnerable to falsification or refutation (Popper, 1972, p. 37). Since the confirmation of a theory is easy to achieve for nearly every theory, he argues that confirmations should only count if they result from risky predictions and are the results of genuine attempts to refute or falsify the theory. A 'good' scientific theory in this view is one that forbids certain things from happening, therefore is (at least theoretically) testable. For example, Eddington's 1917 experiment aimed to test Einstein's prediction that light rays would be bent as they passed through the Sun's gravitational field. This was a genuinely rigorous test and if it had not produced empirical evidence consistent with Einstein's General Theory of Relativity, it would 
have refuted the theory and necessitated its revision or abandonment ${ }^{37}$. However, to accept that the theory had been refuted would also require confidence in the validity and reliability of the observations and measurements.

Any theory that cannot be refuted by any conceivable event is not scientific by Popper's criterion. For example, astrology is characterised by vague predictions that are easily married to the confirming evidence found by hopeful observers. In this view, astrology does not qualify as a scientific theory because the very vagueness of astrological prophecies enables disconfirming evidence to be explained away. In other words, astrological prophecies cannot be severely tested, and therefore cannot be refuted. Whilst this is certainly the case for subjects like astrology, science is not entirely free of such vagueness. Attempts to phrase predictions in science, result in a language that 'embodies a host of expectations about nature and fails to function the moment [those] expectations are violated' (Kuhn, 1976, p. 146). In addition, any theory will contain formalisms that must be interpreted; and ambiguity may appear in either one or both of the formalism or the interpretation (Bunge, 1983, p. 85) ${ }^{38}$.

The formalisms of science are subject to the limitations of language and logic. Consequently, Feyerabend (1976, p. 295) argues that science must become less critical and rational and more anarchistic and subjective. He argues this point not only in favour of the growth of knowledge, but also in relation to social and political concerns:

The idea that science can and should be run according to some fixed rules, and that its rationality consists in agreement with such rules, is both unrealistic and vicious. It is unrealistic, since it takes too simple a view of the talents of men and of the circumstances which encourage, or cause, their development. And it is vicious, since the attempt to enforce the rules will undoubtedly erect barriers to what men might have been, and will

\footnotetext{
${ }^{37}$ Conclusion such as this one, assume that both the experiment design and the instruments were sound. These assumptions may be tested by varying the experimental design, using different instruments, and repeating the experiment at different times and from different places. Similar results can be taken as confirmations of the original experimental results. Vastly differing results might constitute a refutation or the need for further investigation.

${ }^{38}$ Later in this thesis, I will attempt to show that these ambiguities of even greater significance occur in the propositional attitudes, which are expressions of human intentionality.
} 
reduce our humanity by increasing our professional qualifications (Feyerabend, 1976, p. 311).

Science is subject to social conventions and norms as much as any other human pursuit. In Feyerabend's view, the values we hold are more important than the facts we perceive.

The perpetration of scientific fraud may be explained in terms of distorted values, that is, in moral or social terms. Apart from the tendency for researchers to interpret data in ways that support their preconceptions, presuppositions and pet theories, there is also the temptation to tamper with data. Late in 2005, a Korean scientific researcher was found to have been fraudulent in the reporting of his stem-cell research findings. This was reported in the media as undermining confidence in the stem-cell research findings, as published in the May 2005 issue of the journal Science (Chandler, 2005; Reuters, 2006). The moral actions of researchers cannot be separated from the 'facts' they put forward. Similarly, the degree of confidence placed in any reported findings is related to the value placed on their validity.

Despite the possibility of fraud or error, and other problems that may arise in the growth of knowledge, some believe that the achievement of inter-subjective agreement, through social processes, guarantees the growth of scientific knowledge. An article published in New Scientist (Radick, 2005) questions this view. For example, we may wonder about the alternative histories that may have arisen if Newton abandoned science for some other pursuit, Darwin did not sail on the Beagle, or Einstein did not have his 'miracle' year. If social processes guarantee the growth of knowledge, then none of these men, in themselves, could have been important to the growth of science. Improvements in scientific knowledge may be put down to evolutionary processes but to make such a claim is to move beyond science into metaphysics. The social processes of scientific culture may count for more than the actions of individual scientists, despite the 'hero' status we may give to some in the guise of Nobel prizes and the like. Although the natural sciences may be of assistance in explaining the influence of human cognition in culture, science alone 
cannot account for the influences that affect science itself because of the problems of self-reference and the limitations of scientific investigation.

That which cannot be explained by science might be explained by philosophy or mathematics. Caution is needed here however. The false application of scientific or science-like theories to social engineering has led to significant excesses in the past. For example, the attempt to build a Nazi super-race according to the claimed superior characteristics of the 'Aryan' arose from error - as did the more recent use of economic rationalism to justify the exploitation of the weak by the powerful (Gijsbers, 2005). Similarly, the contemporary debate about 'Intelligent Design' stems largely from the status it is given by some as a science, and by others as metaphysics in the form of a philosophical or religious theory (Zwartz, 2005).

Whilst science holds the most promise for our knowledge of human cognition and learning, we must be careful not to extend scientific knowledge beyond its reasonable bounds. This is an error made when unjustified conclusions are drawn from so called 'brain-based' research and applied to theories of learning (See OECD, 2001; Hall, 2005 pp. 14-18). Whilst it is important that our theories about human learning and schools as organisations cohere with science, we must be careful not to 'reinterpret' the findings of science to align them with preconceived views of the world.

\subsection{The place of experience and explanation}

The interpretation and reinterpretation of theories relies on the meaning of the sentences that describe and explain those theories. This is the case for theories of organisational functioning and leadership.

Meaning may be conveyed formally through the literal communication of logical argument, or informally through non-literal forms of communication. Difficulties arise when attempts are made to pin down the meaning of all the words and sentences in a colloquial language. It is not possible to ascribe universal meaning to words due to the various contexts in which they are used, and their propensity to 
ambiguous interpretations. Problems such as logical confusion, self-reference, and the distinction between the use and the mention of a word also render the meaning of sentences ambiguous or unsolvable ${ }^{39}$.

The philosopher Ludwig Wittgenstein and writer Lewis Carroll (Charles Lutwidge Dodson), who Paulos (2000, p. 4) refers to as 'an unlikely pair of men', were both concerned with nonsense, logical confusion and language puzzles ${ }^{40}$. However, whilst Wittgenstein was apparently 'tortured' by these things, Carroll seemed delighted by them, as is apparent in the following extract from his book, Alice's Adventures in Wonderland:

"Of course not," said the Mock Turtle: "why, if a fish came to me, and told me he was going on a journey, I should say with what porpoise?" "Don't you mean purpose?" said Alice'. 'I mean what I say,' the Mock Turtle replied in an offended tone (Carroll, 1984, p.122-123).

In this example, the description of a situation ('with what porpoise') is being confused with its explanation ('don't you mean purpose?'). Similarly, epistemology attempts to explain what we can know and how knowledge may be obtained. This is not the same as describing what we know and how we obtained that knowledge.

Attempts to construct universal explanations, independent of time and place also pose difficulties for logical reasoning. Here, I draw on another example from Alice's Adventures in Wonderland:

And the Gryphon added, "Come; let's hear some of your adventures." "I could tell you my adventures - beginning from this morning," said Alice a little timidly: "but it's no use going back to yesterday, because I was a different person then" (Carroll, 1984, p. 122-3).

In Carroll's story, Alice was a different person simply because she was in a different world altogether from the one she had left and this affected her very identity.

\footnotetext{
${ }^{39}$ The use-mention distinction, as Paulos (2000, pp.40-47) explains, concerns the distinction between object-language (statements within a formal system), and metalanguage (statements about a formal system or about the statements contained within in it).

${ }^{40}$ Here, Paulos refers to the writing of Pitcher (1965).
} 
Changes in situation bring about changed relations and experiences, perhaps even changes of identity. Similarly, epistemology is affected by the contexts in which it is applied. As Popper said:

Of course, we cannot give up either Parmedian rationality - the search for reality behind a phenomenal world, and the method of competing hypotheses and criticism - or the search for invariants. But what we ought to give up is the identification of the real with the invariant (Popper, 1998, pp. 200-201).

Attempts to create a value-free epistemology - one that is independent of the culture and context in which it is used - have not been successful. In philosophy, logical positivism and its relative, logical empiricism, are examples of such failed attempts. In education, cultural and contextual differences are evident in the knowledge employed by school principals, teachers, parents and students. Cook (2006), for example, highlights differences in the ways these groups process the data provided from testing programs. Whilst parents may consider external assessment data valid in guiding what is known about student literacy, teachers are much more mistrustful and dissatisfied with such data. Cook argues that until teachers can connect that data to their day-to-day work with students, it lacks meaning for them:

It's much easier to engage teachers with the meaning of test data when you're working with population testing data: you're talking to them about "their kids" and their teaching program (Cook, 2006, p. 10).

The knowledge of teachers also affects the way they apply data to the evaluation of their own teaching practice.

Despite the fact - or maybe because - they're surrounded by a sea of data on student behaviour and performance, there is no automatic and universal adjustment to their teaching practice in response. Why? (Axworthy, 2006, p. 12).

Perhaps the answer to Axworthy's question lies in the contextual relevance of knowledge claims and the values held by those who make those claims. The valueladen complexities of the work done by school principals and teachers do not lend themselves to simple explanations. Their work takes place in the phenomenological 
world of signs and symbols as well as the physical world of buildings, artefacts, technologies and people.

In phenomenology, the truth of a simulacrum arises in the mere appearance of reality, whereby, perceptions of leadership arise from the representation of signs, which are symbolic representations of reality (See Baudrillard, 1988, pp.166-184; Boje and Rhodes, 2005). Hence, the representation of representation gives rise to the hyperreal, which stands over and above any physical reality ${ }^{41}$. This kind of hyperreality enabled George Clare, an Austrian born Jew, to compare his personal claim to Britishness to that evident in the leadership of Sir John Burgh, KCMG. Burgh, a Viennese refugee who arrived in Britain in 1938 aged twelve, was later appointed Director-General of the British Council' ${ }^{42}$, thus becoming 'the Chief Apostle of the British Way of Life' (Clare, 1989, p.187). Similarly, the Ancient Greek philosopher, Isocrates, announced that to be Hellene was no longer due to the physical reality of race but to the reality of mind and culture (Neserius, 1933).

To explain leadership identity entails much more than a mere description of hereditary biological descent. This is not surprising, because explanations are generally much more complex than pure descriptions. In Carroll's Adventures of Alice in Wonderland, Alice finds herself faced with very different opinions about the desirability of explanations:

"Explain all that," said the Mock Turtle. "No, no! The adventures first," said the Gryphon in an impatient tone: "explanations take such a dreadful time" (Carroll, 1984, p. 122-3).

Explanations are important, but it is tempting to settle for the 'adventures' of experience because the deeper explanations of theory require a great deal of work for their construction.

\footnotetext{
41 The appointment of the clown, Ronald McDonald, as Chief Happiness Officer of McDonald's Corporation as announced in the McDonalds 2005 Annual Report, is an example of a simulacrum. Here, the simulated leadership identity of the clown stands as a sign that draws its meaning from symbols of corporate health (such as increased market share, higher capitalised value, etc.), which are themselves representations of reality.

${ }^{42}$ See http://www.britishcouncil.org/history-who-chief-executive.htm, retrieved 15 January 2008.
} 
In conclusion, in this chapter I have argued that a naturalistic coherentist epistemology offers a suitable epistemology for the construction and evaluation of theories to explain organisational learning in schools. By utilising the superempirical values of conservatism, simplicity, consistency, comprehensiveness, fecundity, explanatory unity, refutability, and learnability, this epistemology avoids the errors of logical empiricism and rationalism and facilitates the global coherence of theories. 


\section{Chapter 3: $\quad$ Methodology}

The three worlds of inquiry of Popper and Eccles (1983, pp. 3-50) form the background of the research methodology, with coherence being sought through philosophical and methodological triangulation. The methodology employs heuristic and hermeneutic methods, and employs a theoretical analysis of naturalistic theories of human cognition as they relate to leadership and organisational functioning; the hermeneutic analysis of leadership narratives obtained from fictional, historical, theoretical and case study texts. The collection, interpretation and analysis of case study transcripts obtained through interviews of formal positional leaders in a single school, are an important part of the methodology.

\subsection{Introduction}

This study is a philosophical investigation of the concept of distributed leadership in schools. Here, I build on the discussion of epistemology presented in Chapter 2, to explain the methodology applied to this study. The methodology framework to be discussed employs both heuristic and hermeneutic methods.

Hermeneutics is 'neither theory alone, research alone, or practice alone' (Nakkula and Ravich, 1998, p. 22). It is a search for the essential meaning of being rather than a systematic search for truth (after the phenomenology of Heidegger, 1962). As life experiences are organised as text, the meaning of human being is revealed by the decoding of the signs and symbols of the text (after Ricouer, 1981):

\footnotetext{
"Pooh," he said, "where did you find that pole?"

Pooh looked at the pole in his hands.

"I just found it," he said, "I thought it ought to be useful. I just picked it up." "Pooh," said Christopher Robin solemnly, "the Expedition is over. You have found the North Pole!" (Milne, 1940, p. 123).
}

The comedy of A. A. Milne's children's story relies on the meaning of being as portrayed through the symbol of expedition and the sign embodied in a found pole. The character, Christopher Robin, who is the author of the expedition, symbolises leadership as he organises provisions for eating, a pop-gun to protect against an 
ambush and 'a long line of everybody'. In the case of the anthropomorphic character, Pooh, his discovery of the 'North Pole' is bestowed as leadership by Christopher Robin because it signifies the culmination, or final explanation, of the expedition.

Research is a means of discovery and explanation, fashioned to expand the reach and depth of human knowledge. The tools and methods of research are artefacts produced by people situated in particular environments, cultures and times in accordance with particular world views and theories. Whist it may seem obvious to say that research requires one or more observers; developments in science have raised deep questions about the dependence of observers on the universe. The General Theory of Relativity, for example, assumes the existence of observers; and according to the Copenhagen interpretation of Quantum Theory, the very act of observing an electron or photon determines whether it will act as a wave or as a particle $^{43}$. As Davies (2006, pp. 295-303) explains concerning the relationship between observers and the universe, there are a number of meta-theories being given serious consideration by scientists and philosophers, including causal-probabilistic theories and quasi-teleological theories.

Causal-probabilistic meta-theories include the absurd universe, the unique universe, and multiverse meta-theories. The absurd universe meta-theory denies the possibility of any underlying coherence or purpose to the universe so that the emergence of life can be no more than an accident. In this view, cognition is understood to have risen from the chance processes of evolution in such a way that 'brains' are able to recognise patterns. This pattern recognition capacity occurs despite the existence of patterns in the universe that do not resemble the patterns of the everyday world; they also arise for no particular reason. Similarly, the unique universe meta-theory, whilst it proposes a deep underlying coherence in the universe that will eventually enable a unified theory of everything to be formulated, denies

\footnotetext{
${ }^{43}$ If there is a boundary between the quantum microworld and the classical macroworld, we as yet have no way to determine where that boundary lies. Whilst the scenario put by Erwin Schrödinger in his cat in a box has generated much debate, its absurdity has never been resolved. Consequently, the entanglement of the quantum micro and classical macro worlds remains, at least, a theoretical possibility (White and Gribben, 1993, pp. 215-216)
} 
that there is any purpose to the universe. The implications of such a theory are that the universe is the way it is simply because there is no other alternative. In this scheme, the existence of life, and observers, is simply an insignificant coincidence. Finally, the multiverse meta-theory says that there are many parallel universes and the reason why this universe is so finely tuned for life (and observers), is simply because of the existence of many other universes that are not fit for life, as we know it.

In contrast, the quasi-teleological meta-theories permit the inclusion of purpose. Their teleological character arises when a final outcome or goal is given as the reason for the events that precede it. These meta-theories include the intelligent design, fake universe, life principle, and self-explaining universe theory. The intelligent design meta-theory says that the universe is the way it is simply because a grand designer planned it that way. This view reduces the questions of why the universe is the way it is, or what it is that constitutes the universe, to the question of who or what designed the universe, and for what purpose. In a similar vein, the fake universe meta-theory proposes that we are living in a massive simulation, a universe sized Matrix governed by an unknown super-mind. The life principle meta-theory postulates an inbuilt principle of the universe that ensures that the universe will evolve towards life and mind. In a similar vein, the self-explaining universe metatheory suggests the existence of backwards-in-time-causal-loops through which the universe explains itself. According to this view, the universe exists to be observed. It therefore brings observers into existence to observe that it exists.

Since the reach of science does not extend to the adjudication of such metatheories, it seems that one must choose between them on non-scientific grounds. Many scientists would choose the absurd universe option simply because it provides the least bother and sidesteps the questions that science cannot satisfactorily deal with. Others may be content with choosing variations of the other theories for ideological or religious reasons, or simply because those views 'feel right' or appeal to the everyday views of folk psychology. 
To choose one of the causal-probabilistic theories is to acknowledge that purposeful actions are actually not possible, and therefore that leadership is not possible. If leadership is simply a series of accidents, it can have no principled or moral influence; and if it has causal force, then it must be possible to explain it in terms of something like the strong, weak, electromagnetic and gravitational fundamental forces, with a similar degree of reliability and universality. However, to choose a quasi-teleological theory is to accept that the origins of purpose and the purposes of leadership lie outside the bounds of empirical investigation. Since this study investigates leadership, quasi-teleological meta-theories are 'on the table', together with causal-probabilistic meta-theories, as possible contenders for explaining the part of observers in the universe, and therefore the part of observers in research.

\subsection{The theory-dependence of research methods}

From a naturalistic standpoint, the theories we hold determine what may be observed and how observations may be interpreted. Hence, school attendance rates of students may be observed and analysed objectively in accordance with the theories underlying roll-marking technologies, and theories that relate educational progression to student attendance rates. Similarly, from the standpoint of phenomenology (Smith, 2005), the background data of subjective experience affects the way we perceive essences or qualities. The essence or qualities of leadership may be perceived against the background circumstances of the experience, which include the previous experience of witnesses.

The views we have of reality have a profound effect on the way we participate in school life and the way we evaluate the purpose and practice of schools. Consider the following four quotations. All are responses to the same question: 'Would you say 
the school community here has a shared vision of what you hope to achieve together in the school, and if so, what would you say that shared vision is? ${ }^{44}$

\section{Principal:}

No, I don't think [the school community has] a shared vision at the moment. I think that as we work towards our next school charter, again, whilst I've had increased parent participation. It's because we have more articulate parents coming into the school (Interview B5).

\section{School Council President:}

A large part of the school community would not have a vision. They would be happy to go along with the decisions made for them by the school. But on the other hand, there is a group of parents who have a strong vision; it's an environmental vision (Interview B6).

\section{Assistant Principal:}

At the moment with the community that we've got now, the vision is the literacy and numeracy thing will happen (Interview B7).

\section{Leading Teacher:}

I'd say [our] shared vision is for, probably, a whole education, a rounded education. Not just, necessarily, an academic education but [one that includes] all the different areas [of learning] (Interview B8).

The differences in these responses may be due to the different standpoints of each respondent and their particular interpretations of the state-of-play in their school. Interpretations of this state-of-play may also be influenced by the world-views of the respondents and the different ways they construe knowledge.

Whilst the ultimate purpose of educational research is a justified theoretical view of educational practice, the methods of research are both constrained and enabled by

\footnotetext{
${ }^{44}$ The background of these transcript extracts is explained later in this chapter. They are introduced here simply to illustrate the point being made at this stage.
} 
the epistemological assumptions and phenomenological viewpoints of both researchers and those who inform researchers. Hence, methodology may be understood as the practical outworking of epistemology. We can evaluate something only in the light of what we understand it to be and by employing the knowledge categories, we possess at the time.

According to Kaplan (1999), concerns with methodology have lessened as more and more researchers simply do their work rather than devoting time and resources to justifying the way they do it. However, a traditional empirical methodology has not been followed in this study, as is explained in this chapter. In keeping with a naturalistic coherentist epistemology, a theory about what constitutes a methodology is presented here as such a theory is central to the methodology itself.

\subsection{Methodology explained}

Methodology draws on several branches of philosophy including epistemology, ethics, semantics and ontology. It is concerned with problems such as:

- What types of theory should we seek to construct?

- What is the most ethical way to advance knowledge?

- What meanings should be attached to the terms employed in the inquiry?

- What are the objects of the inquiry?

Hence, value judgements and procedures feature in the solution of any methodological problem.

Methodology is concerned with the resources and limitations of research methods in relation to both concrete and abstract contexts of inquiry. Methodology comprises a technical aspect, which is concerned with the practical procedures of research, and a philosophical aspect concerned with the analysis of concepts. Sharp distinctions between the three categories of technique, methodology and philosophy are difficult to find. Attempts to specify the "right method" in educational studies, as a field of social science, is at odds with the 'scientific temper, which is open to whatever 
techniques hold promise for the inquiry at hand' (Kaplan, 1999, p. 79). Dogmatic approaches to methodology can take on a mythical nature, closely related to the semantic myth of necessary definitions. According to this mythological view, the effectiveness of research depends on particular instruments of investigation.

To assert 'right' methods is also to assert the use of 'right' terms in describing the method, but the methodological myth gives undue weight to particular methods and the semantic myth gives undue weight to the clarity and precision of definitions. Whilst scientists readily agree that the terms in use must bear some relation to observations, agreement about the characteristics of that relation have not been forthcoming, principally because of a commitment to scientific realism (Boyd, 2002). Widely differing operational definitions may yield substantially the same results. The purpose of operational terms is to eliminate problems of meaning; however, these problems reappear when symbolic operations are used to relate operational terms to observational terms. Operationalism fails because meanings arise from the complex interplay of intuition, imagination, language, theory, and context, not from definitions (Bergman, 1954; Lindsay, 1954; Seeger, 1954). Hence, operational definitions are not employed in this study.

\subsubsection{Three worlds of inquiry}

Any inquiry that seeks to explain leadership or organisational functioning must deal with the complexities posed by the folk-psychological world of human intentions and dispositions. Of equal importance is the physical and artefactual world of educational organisations (such as locality, transport, buildings, computers, books and whiteboards). Finally, no theory of organisational functioning can hope to succeed if it does not take into account the theoretical products of human endeavour. In schools, this theoretical world includes cultural, historical and professional traditions; works of art, music and literary writings; educational policies and curriculum documents; the disciplines and teaching practices that constitute the learning experiences of students; the theories, software, and design principles of technological innovations; and the cultural and intellectual products of school 
communities. The physical, folk-psychological and theoretical worlds constitute the three worlds of human inquiry proposed by Popper and Eccles (1983, pp. 3-50).

These three worlds provide a simple framework for the explaining the evolutionary emergence of theories from human endeavours, and the emergence of human endeavours from the real world. Described in simple hierarchical terms, World 1 (The Objective Real World) gives rise to World 2 (The Subjective Folkpsychological World), which in turn, gives rise to World 3 (The Objective Theoretical World) (See fig. below). However, this conception should be understood holistically so that the distinctions between the three worlds are blurred. The value of the three world conception is that it acknowledges the possibility of supervenient and emergent relations. For example, architectural theories may supervene on the perceptions of architects, who consequently supervene on the architectural possibilities of school buildings. Similarly, certain theories about the impact of building design on educational outcomes may emerge from the experiences of teachers, which in turn, may emerge from the physical arrangement of the school buildings in which they work.

\section{Figure 3.1 Three worlds of inquiry}

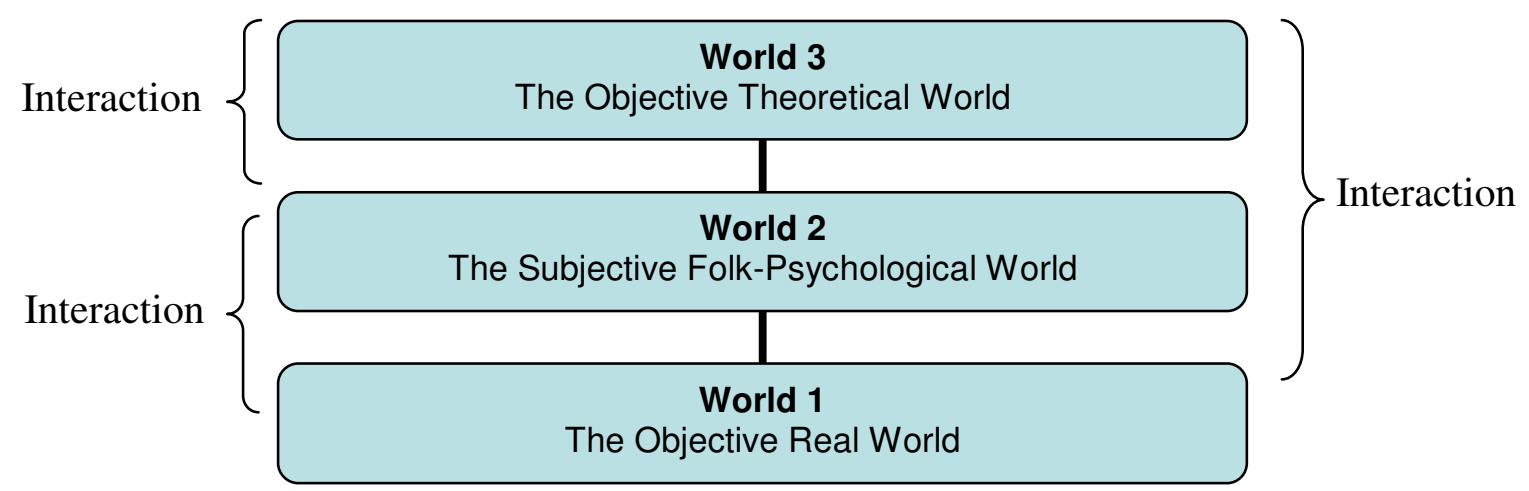

According to Keeves (1999, pp. 4-7) this three world conception provides a means for explaining the interaction between research and practice. It also illustrates the value of multiple methods of investigation in educational inquiry. Each method 
provides access to particular types of interactions within and between the three worlds. Philosophical and theoretical methods arise within World 3; heuristic, hermeneutic and narrative methods arise in World 2; and experimental, case study and ethnographic methods emerge from World 1. Research methods may also be explained in terms of the interactions between these worlds. For example, heuristic methods may be understood as a psychological (World 2) approach to shaping the real world (World 1) and explaining it theoretically (World 3).

This study began with an objective (World 3) theory of parallel distributed processing as a framework for describing human cognition and data processing in schools. As part of a Master of Education Research Study, the author commenced a case-study investigation (World 1) of this World 3 theory. Through the initial analysis, the author came to understand that the subjective folk-psychological world (World 2), and its relationship to World 1 and World 3, was of crucial importance to understanding the importance and scope of the interview data collected in the case study. At the time, the publication of texts such as Hutchins (1995), Clark (1997), and Strauss and Quinn (1997) also marked an important shift in the scope and reach of parallel distributed processing in cognitive theory. In response, the focus of the study shifted heuristically to the examination of parallel distributed processing and leadership in schools, and the title 'Distributed Leadership in Schools' was adopted. This new focus was adopted for a doctoral research project that now employs a naturalistic coherentist epistemology. The original interview data are retained to supplement a much wider narrative analysis of leadership. This shift in the focus and character of research, as described here, was carried out by means of a heuristic method.

\subsubsection{Heuristic methods}

A theory of heuristic methods is explained here because it is central to the method employed in this study. Heuristic methods entail the problem-solving capabilities of humans. They may be suitable for problem-solving processes that require the classification of patterns in problem situations, applying intuitive routines to those problems, evaluating the outcomes, and recognising new problems as they 
emerge. When an algorithm cannot generate a solution to a problem, a variety of search strategies may be employed to improve the chance of finding an answer. These search strategies are called heuristic devices, or simply heuristics (Hill, 1981, p. 251).

Heuristic methods may include a range of approaches including geometry, algorithms, axiomatic method, intuitive methods and inspiration, general rules applied to any problem, and special rules that apply to specific cases. They also constitute rules of thumb; bits of knowledge that are useful for making selections and judgements. Whilst useful for solving a wide range of problems, heuristic methods do not guarantee solutions. The suitability of a particular heuristic strategy depends on the type of problem to be solved and the context to which it is applied. On their own, heuristic methods do not comprise a coherent body of methodological knowledge (Newell, 1983, p. 210), simply because they cannot be applied according to universal principles or rules.

According to Newell's (1983) interpretation, the heuristic method may involve the following steps:

1. Understand the problem;

2. Devise a plan;

3. Carry out the plan;

4. Examine the solution.

This process was applied informally to the meta-problem presented by the choice of research question, as explained in the following paragraphs.

An initial research question was adopted that involved the computational analysis of data obtained in a school setting, with a view to the data analysis informing highlevel decision-making within the school. Artificial neural networks, utilise parallel distributed processing and can be implemented on a parallel architecture or simulated in the sequential series architecture common to personal computers. Computational methods of data analysis constitute formal heuristic methods; they can be described 
theoretically according to the application of computational rules or architectures (World 3), but the manner in which these methods are used will depend upon the purposes of the researcher. The apparent inability of computer applications to deal with a wide range of complex problem-solving situations independently of human input seems to indicate that they lack important capacities that are available to humans. The human ability to assign value to entities stems largely from our emotionally weighted rationality.

Human subjects are capable of serial and parallel distributed processing (World 2). The nervous system operates in a parallel distributed manner whereby information processing occurs via massively interconnected neurons. Yet we are also able to manipulate symbols (such as in the print on this page) in serial sentence fashion. Whilst attempts may be made to describe heuristic methods with sentences, many human problem-solving capacities are difficult if not impossible to describe fully. This may be because those who employ such methods do not have conscious access to the cognitive processes that enable them to do so, or because the relevant processes are difficult to express through the sentential arrangement of symbols. This became apparent in the analysis of the interview data obtained in school interviews.

Human motivation influences the development of a subject's verbal and nonverbal problem solving activities and the development and regulation of goals. Tikhomirov (1983) reports an experimental study in which indicators of subjects' emotional states were monitored whilst solving problems. The study concluded that 'an objectively right solution of a complicated mental problem cannot be obtained without emotional activation, although this activation does not yet provide by itself the objective rightness of results' (Tikhomirov, 1983, p. 161). In other words, emotional responses are important to the formation and revision of goals that lead to a solution; they enable the meanings of elements in the problem situation to be identified and acted upon. Though indicators of emotional activations or convictions appear to be present in the interview transcripts, the objective rightness of any interpretation of the data cannot be determined from the transcripts alone. 
Two other capacities that appear important to informal heuristic processes are the ability to use analogy and metaphor, and the ability to employ humour to set the boundaries of a problem situation (Minsky, 1983). Both of these capacities may be explained in terms of frame (or schema) substitution and the suppression of ineffective or destructive thought processes. The use of analogy and metaphor requires that the shared principles of two frames of thought be known so that one frame can be substituted for the other. Similarly, humour often relies on the surprise that arises from an unexpected frame substitution. However, analogy, metaphor and humour also require the presence of negative, anecdotal censors to suppress ineffective or destructive thought processes. These censors involve the emotions together with any other cognitive processes that are necessary for such censorship to occur. At various points in this thesis, I have used analogy and metaphor with the aim of illustrating the scope of the research problem, as I understand it.

Another emotion related feature of informal heuristic processes is inspiration. According to van der Waerden (1983), problem solving involves both thinking and inspiration. Here, thinking means more than making logical deductions from that, which is already known, but also includes plausible inference, thinking by analogy, thinking in visual pictures, and any other kind of conscious deliberation. Inspiration is that which comes to us suddenly so that it 'falls' into the mind. Its source may be unknown, yet is often accompanied by emotional signifiers. Inspiration often arises in connection with intense periods of conscious work on a problem and seems to indicate that a similar effort of unconscious work has also been done. Hence, inspiration may be understood as that which arises from unconscious cognitive processes. This assumption, though speculative, is plausible given what we know about human cognition; that is, that parallel distributed processing systems do not readily reveal, from within their architecture, how they reach particular outcomes. It seems that meaning has as much to do with the cognitive processes occurring below the level of consciousness as with conscious cognitive activity. After much work has been done, heuristic analysis can bring to the surface, some of the previously inaccessible, tacit knowledge of subjects or researcher. This can be particularly 
important to the identification of meaning in texts, particularly when those texts exhibit emotional content.

\subsubsection{Hermeneutics}

'If there's no meaning in it,' said the King, 'that saves a world of trouble, you know, as we needn't try to find any' (Carroll, 1984, p. 148).

Hermeneutics is a method for finding meaning in texts. Many attempts have been made in philosophy and science to remove the need for the interpretation of meaning, but without enduring success. It seems that meaning is central to the interpretation and understanding of experience. Hermeneutic method was employed in this study through the interpretation of leadership texts drawn from literature and interview transcripts.

Hermeneutics is the theory and practice of interpretation and understanding, or Verstehen (Ödman, 1988). The hermeneutical approach is appropriate to the study of educational organisations because it provides a means for interpreting the various features of educational practice. Hermeneutics may be understood in the first instance as the existential analysis of language, communication and dialogue as it involves the interpretation of meaning in life-styles, conventions, myths, symbols and non-literal language forms.

Hermeneutics employ the criteria of inter-subjective meanings, preunderstandings, and interpretations of reality to control intersubjective meaning. The interpretation of meaning may be checked by comparing the interpretations made by a number of people. However, according to Ödman (1988, p. 68), that is seldom necessary because the hermeneutic circle, by which the parts and the whole are checked against each other, provides a reliable means for validating interpretations.

An interpretation of a text cannot be fully accepted unless it satisfies two criteria: It should include all the available information and be the only interpretation left to explain the events or phenomena under investigation. These two criteria may be used 
to interpret texts in a literal or non-literal fashion. Nevertheless, it can be difficult to know when the first criterion is satisfied and seldom is it possible to satisfy the second. In the case of literal texts, interpretation is aided by the stability of meanings and the use of logical analysis. In the case of non-literal texts, an attempt may be made to reconstruct the world of the text.

The process of determining immediate meaning relies on a long and reliable tradition of intersubjective interpretation. Feelings, emotions and thinking may be interpreted as internal events that leave traces in memory, attitudes and actions. Hence, it may be possible for a triple analogy to be constructed between the literal meaning of texts and the "immediate" meaning of external and internal events. However, immediate meaning must be clearly distinguished from meaning created within a broader social context, which itself constitutes another level of interpretation. This level of interpretation is concerned with the symbolic and cultural messages underlying a feeling or action, a text or its world (Katz, 2005b). Such messages arise not from the logic of sentences or observable events, but from a broad "grammar" of human consciousness and culture. There are no absolute standards for making these types of interpretations; however, the use of the hermeneutic circle is the central theme in hermeneutics (Ramberg \& Gjesdal, 2005).

To Ricoeur (1981, p. 221), the hermeneutic circle is an 'insuperable structure of knowledge' that constitutes the interdependence of explanation and understanding. This correlation expresses Ricoeur's opposition to structuralist interpretations of texts together with his advocacy of depth semantics, by which depth interpretation arises from the personal commitment of the reader. Here, the risk of subjective bias is dealt with, not in terms of "immediate" meaning or an emotional identification with a mental intention, but by the mediating function of the text in disclosing and appropriating a particular world.

Investigation in the natural sciences is cumulative in the sense that meaning cannot be truly understood unless the instruments and knowledge involved in its emergence are known. This has the effect of rendering much of scientific experience mysterious to the lay person and inaccessible to common experience (Oppenheimer, 
1966, pp. 24-25). However, the mediating function of text, rather than undermining the objective function of a social science, serves to provide access to what is essential in reality. Nevertheless, the paradox of self-reflection appears in this analysis, according to Ricoeur who says:

all objectifying knowledge about our position in society, in a social class, in a cultural tradition and in history is preceded by a relation of belonging upon which we can never entirely reflect (Ricoeur, 1981, p. 243)

Science is the critique of ideology, but in the human sciences, this critique can never be separated from the basis of belonging. That is, the critique of ideology, though begun, may in principle, never be completed. Whilst knowledge is always in the process of separation from ideology, ideology always remains the code of interpretation. This code is expressed in narrative.

Narrative analysis has been used in the study to illuminate the concept of leadership. Narrative has been employed as part of the scientific endeavour for centuries. The meaning of the word, 'science' is itself dependent upon the significance of a set of activities attached to that term. Popular and fictional texts utilise culturally familiar images or representations to evaluate the purpose of science in social life. Even highly technical scientific texts, produced for an elite audience in order to criticise the writing of its own members, may offer previously unforseen possibilities.

Ormiston and Sassower (1989) offer accounts of the importance of narrative to the development of scientific ideas, citing in particular, Francis Bacon's (1620) New Organon, Galileo's (1638) Dialogues Concerning Two Sciences, Thomas Hobbes's (1660) Leviathan, Jean-Jacques Rousseau's (1762) Emile or On Education, Mary Shelley's (1818) Frankenstein, and George Orwell's (1949) 1984. According to their analysis, Bacon, Galileo and Hobbes are each interested in presenting an abstract, ideal world. In doing so, they present wholly different sets of conditions for human life. Their narratives take on mythical qualities that go beyond contemporary understandings to a grand narrative of possibilities. 
Attempts to present a unified picture of science in grand narrative or to specify the rules that govern our understandings reveal an absence of universal social knowledge. According to Ormiston and Sassower (1989, p. 23), universal knowledge in the form of an objective standard or foundation, remains a fictive ideal. As such, it remains another story we tell ourselves - one that awaits validation according to our needs and goals. Nevertheless, narrative texts can claim the legitimacy of science if they avoid logical contradiction and their claims to objectivity and truth can be validated (Ormiston and Sassower, 1989, p. 98).

A defining characteristic of fictional texts is that they provide a set of rules or criteria by which their own claims may be evaluated. In adopting the rules of rational discourse as a style of presentation, and experience (or experiment) as the basis of knowledge, Bacon, Galileo and Hobbes also establish the principles that legitimate their texts (Ormiston and Sassower, 1989, pp. 19-26). Hence, the distinction between scientific narratives and fiction is blurred. Similar to the approach taken by Bacon, Galileo and Hobbes, Rousseau sees experience as the basis for understanding nature but, unlike the other three writers, offers a subjective view of nature and its governing laws. To Rousseau, the direct experience of the individual is what counts, not any presumption of objectivity or authority. Shelley's Frankenstein and Orwell's 1984 reorient our understandings of the social significance of science. That is, they rely on a framework provided by science to force a rethinking of our relations with each other, our creations, and nature. Their texts warn against any presumption of a fixed end or goal, or the presumption of fixed, well defined boundaries. Such openendedness is exemplified in metaphor and symbols.

According to Ricoeur (1976, pp. 45-69), two kinds of metaphor occur in narrative. The first kind entails the simple substitution of one word with another and is therefore translatable. The second kind of metaphor, known as a tension metaphor, is not translatable because it creates its own meaning. A tension metaphor has more than emotive value; it is not a mere ornament of discourse because it offers new information. The relation between the literal and figurative meaning of a metaphor also provides a way to understand the extension of meaning found in symbols. Metaphors are simply the linguistic surface of symbols 
Symbols encompass a surplus of meaning; they cannot be exhaustively treated as a conceptual language; that is, the experiences, ideals and understanding that are brought to language in symbols can never be fully passed over into language. As Ricoeur (1976, p. 63) has said, 'language only captures the foam on the surface of life'. Below the semantic surface, lies a pre-semantic world of human experience that resists description.

The mere possibility of symbols, metaphor, and figurative forms being embedded in narratives of leadership and organisational functioning is of great importance to the interpretation of those narratives. This study draws from a large variety of leadership narratives, including but not limited to case study interviews. The philosophical focus of this thesis is one that seeks to illuminate the concept of leadership, so does not assume a literal rendering of narrative texts. The interpretations of school leaders go beyond that which may be discerned by literal or computational means.

Nakkula and Ravitch (1998, pp. 49-55) suggest four criteria for determining valid hermeneutic interpretations of the text of a phenomenological investigation. They base these on a summary provided by Packer and Addison (1989) and their own experience with interpretive research: coherence, external evidence, consensus and practical implications. Coherence refers to the internal structure and believability of interpretive findings. External evidence refers to the relationship of an interpretation to other, competing interpretations. Consensus refers to agreement about the clarity and meaning of data such as interview texts. Finally, practical interpretations refer to the applicability of interpretations to future or existing practice.

In this study, coherence is sought through the interpretation of the interview texts as a single whole; external evidence is applied by comparison with other leadership texts and narratives; consensus is achieved by seeking agreement about the final form of the interview texts; and practical interpretations are evident in the conclusions drawn from the interview data. 


\subsubsection{Case study interviews}

As Merriam (1988, p. 1) says, there is little agreement about what constitutes case study research even though the term case study is a familiar one. Here, the term case study is a means for understanding and interpreting a particular case, which can be an open or a bounded system. The disadvantages of the case study method include the following (Yin, 1981):

- a reliance on intuitive interpretations of meaning;

- the fuzzy distinction between method and findings inherent in the coding of data;

- the heightened sensitivity of research respondents to conclusions drawn from their responses;

- the tendency for researchers to spend an inordinate amount of time constructing readable narratives, rather than simply taking notes; and

- the tendency to report findings as lengthy narratives, which lack a predictable structure.

These weaknesses may be ameliorated by framing the study with a set of questions to reflect the background theoretical assumptions and hypotheses of the researcher. This was the approach taken in this study, with a set of questions prepared prior to the trial interviews, evaluated, and then modified before use in the substantive interviews. In single case studies, the data analysis is assisted if data is analysed according to its fit to recognisable patterns. That way, relations of similarity and dissimilarity between patterns may emerge, so they may give rise to novel patterns in the data. Finally, findings are better reported as a set of answers to openended questions rather than as a continuous unstructured narrative. This serves to segment the findings into chunks that are more readily assimilated by readers, and facilitates their assimilation into a broader, more meaningful, structured, research narrative. 
No one method of data collection is claimed for case study research. A hermeneutic approach was chosen for this study, with empirical data being collected by means of interviews. Here, the purpose of the interview method is to illuminate the phenomena being studied. This has the advantage of revealing the influence of personalities and opinions on a situation, illustrating the scope of situational complexities, and presenting information vividly and in a variety of ways.

A standardised, open-ended interview was the method used for this purpose. This type of interview comprises carefully worded questions, which are put to all of the subjects in a similar way. A carefully prepared interview environment and an accurate, empathetic listening attitude on the part of the interviewer are important in hermeneutic research. It is also important for the researcher to be flexible and free to adjust procedures to respond to the flow of the dialogue. Because the accounts given by subjects may include stories, examples, conversations, metaphors and analogies, the interviews should be recorded and later transcribed. To supplement the interview data, the researcher may also collect documents, which may shed further light on the contents of the interviews. All of these procedures were utilised in this study.

Nisbet and Entwistle (1970) describe the basic principles of a standardised interview. Though they are mainly concerned with the collection of statistically reliable data from a representative sample, some of their principles may be adapted to a small case study. Firstly, the precise wording of questions and instructions is prepared in advance. This constitutes the interview schedule and prescribes the pattern to be followed in the interview. Though a statistical analysis requires a preliminary taxonomy for the classification of respondent's answers, a case study does not. The purpose of a case study is to describe the characteristics of a specific situation. Since that situation may or may not be representative of a general population, it is usually not possible to draw universal conclusions from such a study.

The first stage in preparing an interview schedule involves specifying the aims of the inquiry, identifying some potential factors for investigation, and drafting the research questions. Nisbet and Entwistle (1970, p. 37) describe this stage of the research process as a period of stress for the researcher - a stage of intuitive 
exploration and 'mental uncertainty'. During the second stage, the subjects to be interviewed are identified and the administration of the interviews is organised. The third stage involves a pilot run of the interview procedure, which includes an evaluation of the questions. Here it is important to complete the pilot run with a sample group that is similar to the group from which the research data is to be collected. Obviously, the pilot group should not include any person to be interviewed later. In the pilot study, the interview procedures, questions and the mode of data classification should be given a fair run so that any problems can be readily identified and remedied.

Interview responses may be categorised during the interview according to a predetermined taxonomy or coding system. Alternatively, the interviewer may attempt to reduce interview responses to a set of words or phrases in an attempt to summarise the key points made by the respondent. A third approach is to take a recording of the interviews, from which transcripts are produced for later analysis.

The first method has the advantage of reducing the range of variables to be considered before the interview is conducted, thus making the analysis of data more manageable. Such an approach enables the responses to be tailored to the theory under consideration. However, it also serves to limit the interpretation of the data and poses problems for the interpretation of unanticipated responses. The second method has the advantage of being more flexible, enabling the researcher to classify responses without relying on uncertain presumptions. However, it is also more prone to error and inconsistency because the classification scheme is being constructed whilst the interviews proceed. The third method enables a thorough, but time consuming, analysis of the data to occur. It also enables multiple analyses of the data so a cross-checking or triangulation of interpretation may be conducted from the same data set. Its disadvantage is that it may result in a divergence of discussion, simply because it enables the respondents to answer in accordance with their interests and particular experiences. It is, however, suitable for gaining insights into the theories, values and goals of respondents. This third method is the interview method employed in this study. 
When the interviews are being conducted, it is important to first establish rapport and allow the subject to feel at ease, at least during the early stages of the interview. It is important to state the questions as consistently as possible, with consideration for the developing context of the interview and the non-verbal cues of the interviewee. The interviewer should also listen with interest and allow the respondent to give a complete answer to questions without interruption. Sometimes it may be necessary to restate the question or part of the question, or to clarify its intent, if this helps the respondent to give a relevant answer.

If the interview subjects are comfortable with such an approach, their verbal responses may be recorded and later transcribed into print. This is a time consuming process, which requires that the initial transcripts be checked for accuracy. Verbal statements can may be misheard or misunderstood, especially if the person doing the transcribing is not familiar with the background of the research or with any technical terms or abbreviations used by the researcher and respondents. In this study, the interview texts were initially transcribed from tape recordings by people who fitted that category. Consequently, all transcripts were checked for accuracy and edited by the author, who was familiar with the background of the research and the technical terms and abbreviations in use.

Once the transcripts have been drafted, checked and corrected, transcript narratives may be analysed.

\subsubsection{Narrative methods}

Narrative method is employed in this study for the analysis of narrative texts drawn from literature and interview transcripts. In keeping with a coherentist epistemology, a general theory of narrative method is central to the narrative method utilised in this study. Hence, narrative methodology is described here as narrative thinking.

The single most important feature of narrative thinking, according to Clandinin \& Connelly (2000, pp. 24-25), is that it exists at the intersection of narrative inquiry and 
other forms of inquiry. Here, the narratives under inquiry should be understood as situational narratives. Situational narratives do not sit comfortably within a paradigm, stable inquiry framework or habit - all of which may proceed without taking into account alternative frames of reference. These are characteristics of grand narrative, which is independent of time, context-free and universal. The central features of narrative thinking, however, include emplotment, character, scene, place, time, and point-of-view (Clandinin and Connelly, 2000, p. 26). Hence, the interpretation of situational narrative depends upon the way time, place and other contextual features interact with human beliefs, emotions and intentions. To think of schools according to situational narrative is to view principals and teachers as people who are participants in the changing, dynamically interactive context and culture of school life.

A tension exists between the reductionism of the grand narrative, also described by Schön (1983) as technical rationalism, and thinking narratively, which sits comfortably within Schön's (1983) reflection-in-action. Clandinin and Connelly (2000, pp. 35-47) describe the grand narrative and narrative thinking as different forms of thinking. Whilst grand narrative may be seen to interpret and reduce experience to established theoretical categories, thinking narratively tends to interact at the boundaries of other forms of inquiry to see things 'as they are'. The dependence of grand narrative on the formalisms of established theoretical categories contrasts with the more informal and intuitive approach taken by those who think narratively. To think narratively is to think holistically, beginning with experience at the centre and proceeding outwards to formalisms at the boundaries.

Narrative thinking does not imply relativism (Clandinin and Connelly, 2000, pp. 85-86) by which any interpretation of events is acceptable. Rather, it entails better or poorer interpretations of the evidence or different perspectives of the same truth. In addition, as the data is read and re-read, different details may be noticed, more significance may be apportioned to particular pieces of data, and new connections between data noticed. Hence, thinking narratively allows better accounts to arise from the reading and re-reading of the evidence, and therefore allows for learning. 
As learning occurs through narrative inquiry, the distinction between explicit and tacit knowledge dissolves. Narrative inquiry is not clearly governed by theories, methodological tactics, and strategies (Clandinin and Connelly, 2000, p. 138-168). The categories of formal theories tend to fragment, as the objects of inquiry become intimately known, regardless of whether those objects are individuals or whole situations.

A narrative form, 'something like Aristotle's formal cause or Dewey's end-inview' (Clandinin and Connelly, 2000, pp. 152-153), may guide a research program by shaping the practice of research. Narrative form is itself transformed by the research practice, so is not like a research question or formal objective. The fluidity of narrative inquiry is both its strength and its weakness. It is strength because it allows for the emergence of new understandings and lines of inquiry, and because it does not bind the inquiry to assumptions that may later become a hindrance to learning. It is weakness because the boundaries of investigation are not easily constrained and the inquiry may need to be cut short of a satisfactory conclusion, or be at risk of exceeding its allocated resources.

The distinction between fact and fiction may be muddled and confused in narrative inquiry. The possibility of memory reconstruction, by which some events are ignored and others highlighted or reinterpreted, is present:

\footnotetext{
When a researcher is in the field and a story is told or an event is narrated, we may well wonder about the basis of the story. Did the events described actually happen? How do we know? How does the teller know? The answers to these questions, if the questions are asked at all, will vary depending on the story or event at issue (Clandinin and Connelly, 2000, p. 179).
}

Narrative inquiry is the study of experience, and experience arises from the actions and relations of people in time and place. As such, it has an intersubjective quality that risks the creation of a narcissistic point-of-view. Similarly, there is a risk of 'narrative smoothing,' by which clean, unconditional "Hollywood" plots are created. Such risks may be ameliorated by triangulating narrative methods with theoretical methods. 


\title{
3.3.6 Theoretical methods
}

Theoretical methods are central to the methodology of this thesis. They involve the formulation of generalisations and the building, testing, revision and replacement of theories. As with narrative methods, theoretical methods can suffer from problems of meaning, evident in normative and functional ambiguity, internal and systematic vagueness, dynamic openness and premature closure. They can also suffer from paradox of conceptualisation, by which the formulation of sound theories depends on having suitable concepts at hand, and suitable concepts cannot arise without first having an appropriate theoretical understanding. The resolution of this paradox simply requires that theories and concepts be arrived at by successive semantic and epistemic approximations (Kaplan, 1999, p. 81). In this way, theoretical methods share some similarities with narrative methods. However, because their epistemic structure differs from that of narrative methods, they can be triangulated with narrative methods.

Whilst the structural analysis of narrative does not enable fact to be distinguished from fiction, theoretical methods may ameliorate ambiguities by specifying the relations between the constituent hypotheses of a theory. In some cases, theoretical methods may enable the construction of a structural model to explain the phenomena under investigation, thus enabling the confirmation or disconfirmation of predictions by observation.

Theories and models always have a provisional nature. Their value lies in their ability to guide further investigation:

\begin{abstract}
A theory, it has been said, is a policy, not a creed. It does not purport to provide a picture of the world but only a map. It guides decisions on how best to deal with the world, including the decisions on how to continue fruitful inquiry. It raises as many questions as it answers; the answers themselves are proposed directives for action rather than assertions for belief (Kaplan, 1999, p. 89).
\end{abstract}

When a model utilises a significant symbolic structure it may allow exact deductions and explicit correspondences with reality to be made. Two dangers are presented by such models, however. The first arises when significance is placed on 
features of the model that do not belong to its structure but to the symbolisation of the structure. The second is oversimplification by which significant features of the structure are omitted from the model. It is not essential that theories be presented as holistic models. However, the component hypotheses of a theory may not be easily separated from the whole, simply because the terms used in the hypotheses draw their meaning from the systemic meanings implied by the theory as a whole.

Coherentism requires that theories be evaluated according to their fit to other, trusted theories, regardless of whether those theories are constructed according to formal theoretical methods, narrative methods, case study, hermeneutical methods or heuristic methods. The methodological principle, by which such evaluation can be achieved, is known as triangulation.

\subsubsection{Triangulation}

Heuristic, hermeneutic, interview, narrative and theoretical methods may be drawn together through triangulation. The term, triangulation, is a metaphor for a composite methodology - one that uses two or more methods as a means to comparing findings and adjudicating their theoretical worth. There are four major types of triangulation: data triangulation of time, space and persons; methodological triangulation by which more than one method is used; theory triangulation, by which interpretation is conducted according to more than one theoretical scheme; and philosophical triangulation which employs two or more philosophical perspectives. An investigation may also employ a composite triangulation methodology, by which two or three of these types of triangulation are used.

Methodological triangulation is the combined use of a number of research methods to ameliorate the weaknesses and biases of a single method (Denzin, 1988). Any one method will have its weaknesses and strengths. Social surveys cannot fully portray the respondent's life-world; participant observation may be subject to biased interpretation; interview responses may be inadvertently influenced by the nonverbal cues of the interviewer; and narrative inquiry may be overly narcissistic or solipsistic. In addition, the social world is constantly changing, as are the meanings 
that participants or researchers attach to their social world. No one method will be capable of capturing all of the changing features of the social world being studied. Each method will highlight particular aspects and interpretations of a given situation and suggest its own distinctive lines of further inquiry. Furthermore, research methods are themselves subject to the interpretation of the researcher and context specific factors such as research funding, the availability and cooperation of survey or interview respondents, and practical constraints.

The interpretation of results presents many difficulties. Distortions in the responses of subjects may occur due to Hawthorne and halo effects ${ }^{45}$, and by subjects viewing interviews as some kind of test for which the 'right' answer must be given. Sampling bias may also result in a set of responses being unrepresentative (Nisbet and Entwistle, 1970, p. 157). Statistically small studies have a high probability of returning results by chance alone. During analysis, unusual results tend to catch the eyes of the researcher. Even if analysed statistically, the interesting case may pass a significance test but it will not be possible to know if this happened by chance or not.

The aim of triangulation, then, is to remove bias and susceptibility to chance events by providing confirmatory support for particular interpretations of the data. It also aims to illuminate phenomena under study and reveal the contextual influences that bear upon it. The hermeneutic interpretation of data requires that the researcher enter the centre of the circle of interpretation and therefore to be part of the interpretation. The triangulation of data enables a researcher to do this whilst also checking that the interpretation is appropriate and accurate.

\subsection{The research methods of this study}

Building on the previous discussion of methodology, the methods used in this study are now described. According to Nisbet (1999, p. 73), education research exhibits two functions. The first, which may be known as basic research, is

\footnotetext{
${ }^{45}$ Hawthorne and halo effects are those by which research subjects respond in extraordinary ways to the attention of the researcher, so that the very act of observing changes that which is being observed.
} 
concerned with the long-term creation of a theoretical framework, within which dayto-day issues may be perceived. The second, known as policy-oriented research, is concerned with the more immediate need of dealing with routine problems according to prevailing views and within the current context of educational provision. Though useful for classifying the purpose of a research project, the distinction between these two categories is not a sharp one; each one has an influence on the other.

This research study is of the basic kind, the kind that is designed to contribute, in the first instance, to the creation of a theoretical framework. Education, like other socially related disciplines, tends to absorb new ideas and concepts gradually into popular thought and discussion until a new climate of opinion arises. Whereas academic status tends to be given to those who contribute to theory, educational administrators and politicians tend to respond to research findings that conform to the current popular view, that is, findings that enable the practical implementation of ideas and concepts without radical change to the theoretical status quo. However, the best approach, according to Nisbet $(1999$, p. 74$)$ is for research to contribute to all of policy, practice and theory. This study attempts to do this by making explicit links to theory.

The purpose of the methods utilised in this study is to seek answers to the following questions:

1. To what extent are organisations the outworking of human cognition as described by theories of distributed cognition?

2. To what extent can the functioning of schools as educational organisations be explained in terms of leadership?

3. Given the answers to the previous questions, what can a theory of distributed leadership offer?

The methodology employs a naturalistic coherentist epistemology and the three worlds ontology of Popper and Eccles (1983, pp. 3-50). Though this study focuses on the concept of leadership as applied in schools, it draws on and evaluates theories from several disciplines. It draws on philosophy, as it pertains to epistemology and 
methodology; theories of natural science, as they pertain to explanations of human cognition; narrative theory, as it pertains to leadership; and organisation theory, as it pertains to schools. Together, these theories are evaluated holistically by employing coherence justification.

The purpose of this study is to analyse the concepts of leadership and learning in schools, where learning is understood in accordance with distributed cognition theory. With this in mind, a true explanation of leadership in schools is sought, whereby truth claims are justified according to their coherence with our most trusted theories. The research questions and methods for the analysis of those questions were chosen heuristically. This heuristic method comprised progressive cycles of analysis, problem specification, and resolution.

\subsubsection{Heuristic cycles}

The first cycle, which took place as a Master of Education study, centred on an exploratory investigation of data analysis and organisational learning in schools. The initial theoretical basis of the study related to the suitability of artificial neural networks as a means of data analysis in schools. At this point, the investigation involved a search for similarities between the computational principles and processes of artificial neural networks and the way designated school leaders analyse the information available to them. A qualitative interview method was the principal method used at this stage of the investigation. This is explained in more detail in Section 3.4.3.

The second cycle, took place when the study was expanded and modified to suit a $\mathrm{PhD}$ level study. A literature review had been conducted in relation to organisational learning and interview data had been collected and analysed. The initial analysis of organisational learning theory and the interview transcripts revealed that the theoretical focus of the study was too narrow to yield any meaningful results from the empirical data. Consequently, the focus on artificial neural networks was expanded to one that incorporated concepts from distributed cognition theory (Hutchins, 1995), cognitive theory and culture (Strauss and Quinn, 1997), and the 
interaction of mind, body and world (Clark, 1997). The study evolved from one that examined leadership and organisational learning to one that examined the relationship between leadership and distributed cognition according to a naturalistic coherentist epistemology. Hence, the title 'Distributed Leadership in Schools' was $\operatorname{chosen}^{46}$.

The third cycle involved a further revision of the scope of the study. As argued in other parts of this dissertation, claims to leadership cannot be sustained based on naturalistic analysis (see for example, Lakomski, 2005). It was recognised, therefore, that attempts to infer leadership from a naturalistic analysis were unlikely to be successful. Consequently, it was decided to analyse the concept of leadership narratively - not only through the analysis of a single school situation, but also through a wide-ranging analysis of leadership as portrayed in literature. Leadership narratives were selected from a wide selection of literature sources. These included narratives drawn from children's, military, historical, political, and educational sources. A chance observation of two children on a school excursion also provided an additional leadership narrative, which was included in this analysis. The interview transcripts collected during the first heuristic cycle of this study were included because they were collected in an educational context and provided insights into the narrative world of designated school leaders. Since the roles and functions of the interviewees are accepted as being leadership roles and functions, the identification of the interview transcripts as leadership narratives was a valid one. It is for this reason that the interview transcripts were retained, for illustrative purposes, in the expanded $\mathrm{PhD}$ study.

The fourth heuristic cycle occurred as the distinction between two forms of information - naturalistic and phenomenological - was identified as significant. The narrative schemas of situational narrative, folk psychology and the propositional attitudes present information in a manner logically distinct from that presented through the theoretical concepts of grand narrative, scientific psychology and

\footnotetext{
${ }^{46}$ The Master of Education thesis had not been completed at that time, so the work completed to that point was expanded and subsumed within the $\mathrm{PhD}$ project.
} 
theoretical propositions. However, information space may reveal both naturalistic and phenomenological properties (Chalmers, 1996). The information evident in human emotion may be explained in terms of causal, physiological processes (see Damasio, 1994) and intentional, phenomenological qualities.

The analysis of these concepts comprises a deeply philosophical analysis of leadership as a phenomenological quality, and organisational learning in schools as causal-computational processes. Hence, this final cycle led to the argument that organisational learning in schools is a causal-computational process as described by distributed cognition theory, whilst leadership in schools is a phenomenological essence that is perceived against a background of individual preferences, cultural expectations, signs and symbols. Consequently, my thesis is that organisational learning is amenable to naturalistic analysis, but leadership is not (See Chapter 6 for further discussion of this claim).

\subsubsection{Hermeneutic analysis}

Hermeneutic analysis was an important component of the methodology of this study. As Nakkula and Ravitch (1998) have argued, human lives may be depicted linguistically as text. Consequently, hermeneutic analysis may be applied to phenomenological interpretations of experience as much as it may be applied to literature. It is in this way that the phenomenological understanding of being merges with phenomenological method to enact dialogue between theory, research and practice.

What does it mean to be a leader or to enact leadership? If linguistically constructed texts can be interpreted in a way that approximates the essence of leadership, then texts about people who have been labelled as 'leaders' should reveal something of that essence. That essence (or its approximation) may be seen in other texts - those that do not explicitly identify leaders or leadership. It was in this way that texts were identified as leadership narratives. These texts were analysed and selected extracts were used to construct an extended narrative of leadership in Chapters 4 and 5. The narratives include extracts from personal reflections, 
biographies, autobiographies, historical texts, and instructional or policy texts. Other extracts were drawn from interview transcripts and one was obtained by direct observation.

The interpretation of each extract was conducted by reference to the larger text, context, or situation. Common themes, concerning the essence of leadership, were identified in the texts from which the extracts were drawn. Those themes were presented as major categories of leadership in Chapter 4. Hence, hermeneutical analysis both reveals and utilises approximations to the essence of leadership. In this way, the hermeneutic circle was employed, whereby the whole is understood by reference to the component parts of the narrative, and the parts are understood by reference to the whole. Here, the 'whole' included not only the larger text from which each extract was drawn, but also the socio-historical contexts of the larger texts.

\subsubsection{Structured interviews}

Some of the texts utilised in this study were drawn from structured interviews, conducted with people who had been identified as leaders in a State government primary school. In this case, their status as leaders was inferred from the titles and formal responsibilities of the positions they held in the organisation. Since the substantive interview texts ${ }^{47}$ were gathered from a single school, they qualify as a case study. Some additional information in the form of documents volunteered by the principal of the school, helped to build an understanding of the context to which the interviews refer. The analysis of these texts was conducted in accordance with the hermeneutic circle, so that meanings were inferred from the relationships amongst the texts and the relationship between the texts and the context to which they referred. The relationship between the interview texts and the larger text presented by

\footnotetext{
${ }^{47}$ Here, the substantive interview texts are those that were incorporated into the hermeneutic analysis; as opposed to the texts obtained during the trialling of the interview method, which were not incorporated into the hermeneutic analysis. See the appendices for the full transcripts of the four substantive interview texts.
} 
the socio-political environment (i.e. the prevailing policy environment of State government schools as it stood at the time) was also taken into account.

The interviews were conducted by myself, the author, under the umbrella of the Cooperative Research Project (Cooperative Research Project, 1998). The two schools were selected according to the following process:

1. Returns from the sixth survey ${ }^{48}$ of principals of the Schools of the Future program were examined.

2. Primary schools were chosen from this list because of the relatively small size and less complex organisation of those schools. From these, primary schools who had expressed willingness to take part in a research project were selected.

3. The list was reduced by selecting schools in Intakes 1 and 2 of the Schools of the Future program ${ }^{49}$. Schools from these intakes were chosen because they had undertaken Triennial Reviews in 1997, the year in which the selection process was conducted. The schools in those intakes were the first to be involved in a new process of triennial school reviews, which it was anticipated would involve the subjects in an evaluation of their school and the setting of new directions and priorities. Hence, it was expected that the subjects would have given some prior thought to the organisational progress of the school, and the data analysis conducted as part of the Triennial Review.

4. The list was further reduced to schools located in the Victorian Government South-East Metropolitan Region. ${ }^{50}$

5. Finally, the two participating schools were chosen after they responded positively to a further, more specific invitation, which included a brief overview of the

\footnotetext{
${ }^{48}$ These surveys were conducted as part of the evaluation process for the Victorian State Government's Schools of the Future program. This was a program that devolved control and responsibility for the management of schools from the central bureaucracy to school councils and principals.

${ }^{49}$ Intakes 1 and 2 comprised the first two intakes of schools into the Schools of the Future program. ${ }^{50}$ Schools were chosen from that region because of the proximity of the school to the interviewer's normal place of work. This was necessary because the interviewer was employed full-time in a school and was enrolled in the relevant university course on a part-time basis. In addition, funding was not available to release the interviewer and/or the interviewees from their full-time work responsibilities for the time required to conduct the interviews. Choosing a school in close proximity to the interviewer's normal work location enabled frequent short visits to be conducted.
} 
intended research. Two schools were chosen so that the methodology could be trialled in one school prior to the substantive study being conducted in the other school.

In each school, four school leaders agreed to take part in the study, being the principal, assistant principal, a leading teacher, and school council president. These people were chosen because they held positions that were accepted as being leadership positions in the school. Each position entailed certain powers and a particular sphere of responsibility. However, it is generally accepted by the Victorian Government education bureaucracy, and by parents, teachers and students that the principal is the leader of the school, at least in a formal sense. Since leadership is a disputed concept and perceived by people in different ways, the most straightforward way to obtain the views of 'leaders' is to interview people who have been traditionally or formally identified as leaders in schools. Distributed leadership, as it is conceived in this study, entails the distribution of the cognitive processes of those who enact leadership. Those cognitive processes are amenable to naturalistic analysis, but leadership is not. Hence, the most straightforward way to get a purchase on the concept of leadership, as understood by those being interviewed, is to interview those who have already been identified as leaders by the school system.

Several ethical issues were taken into consideration. Firstly, it was considered possible the subjects may experience anxiety during interviews. The interview questions could have raised issues the subjects had not considered before, felt were too probing, or not felt comfortable talking about. To minimise the likelihood of these concerns arising, the researcher provided background information explaining the purpose of the research, the manner in which it would be conducted and the means by which the research findings would be reported. The subjects were given an assurance that their confidentiality would be preserved by replacing their names and the names of their schools with pseudonyms and removing any secondary references that may point to their identity. The possible benefits of the research for the subjects were also taken into account. It was considered that the interview questions might prompt the subjects to reflect upon their own practice in a way they had not done 
previously. In answering the questions, the subjects may also have found reassurance that they and their schools were dealing well with leadership and organisational issues.

Each was invited to participate in an interview lasting up to one hour. At the commencement of interviews, the subjects were invited to indicate any discomfort experienced and were reminded of their right to withdraw their participation from the research, including any data they had provided, if they wished to do so. Measures were taken to minimise any discomfort the subjects may have felt during or after the interviews.

Open ended, semi-structured questions were chosen to elicit rich detail in the responses of the subjects. As the questions were drafted, the researcher attempted to use terminology familiar to them. Relatively neutral descriptive information was sought early in the interview to assist interviewees to feel at ease. These questions were placed in a category titled, background information. Later questions focused on the types of data collected by the school, the manner in which information is analysed, and how action follows from that information. These questions were drafted then revised after heuristic analysis, conducted according to the relevant theoretical considerations at the time (based initially on an analysis of organisational learning and the concept of the learning organisation as described by Senge, 1990). The questions were then trialled in a pilot study conducted with one of the two selected schools. This enabled the questions and interviewing method to be refined in the light of the responses of the subjects and the heuristic reflections of the researcher.

Following the pilot study, it was noted by the researcher that the participants desired a written copy of the questions to hold and refer to during the interview, and that they would like to have some forewarning of the scope of the questions. Therefore, when the substantive interviews were conducted in the second school, the questions were mailed to the subjects to consider beforehand. The researcher also adjusted his style of questioning to assist the subjects to feel more at ease and answered participant's queries about the meaning and significance of the questions. 
Sometimes, more probing questions were asked in order to elicit further information from the interviewee. If it became clear to the researcher that the subject had misunderstood a question, the question was sometimes rephrased so that the subject better understood its meaning and intent from the researcher's point of view. The category and text of the interview questions are shown in Table 3.1, below.

Table 3.1 Interview questions

\begin{tabular}{|c|c|}
\hline Category & Question \\
\hline $\begin{array}{l}\text { Background } \\
\text { information }\end{array}$ & $\begin{array}{l}\text { 1. How long have you been in your current leadership } \\
\text { position at this school? } \\
\text { 2. Please describe the scope of your current leadership } \\
\text { responsibilities in the school. }\end{array}$ \\
\hline Individual goals & $\begin{array}{l}\text { 3. What do you hope to achieve in your current position as a } \\
\text { leader? } \\
\text { 4. What sorts of data or information do use to assess your } \\
\text { progress in achieving this? } \\
\text { 5. How has this information influenced your personal } \\
\text { achievements and the way you operate? }\end{array}$ \\
\hline $\begin{array}{l}\text { Organisational } \\
\text { values and } \\
\text { vision }\end{array}$ & $\begin{array}{l}\text { 6. Would you say the school community here shares common } \\
\text { values or beliefs about education and schooling? } \\
\text { 7. If so, what would you say those shared values are? If not, } \\
\text { what would you say the main areas of disagreement or } \\
\text { non-agreement are? } \\
\text { 8. Would you say the school community here has a shared } \\
\text { vision of what you hope to achieve together for this } \\
\text { school? If so, what would you say that shared vision is? } \\
\text { 9. What sorts of data or information does this school collect? } \\
\text { 10. How do you analyse this data to monitor the achievement } \\
\text { of the school's vision? } \\
\text { 11. How has this information influenced the school's } \\
\text { achievements and the way the school operates? }\end{array}$ \\
\hline
\end{tabular}




\begin{tabular}{|c|c|}
\hline Category & Question \\
\hline $\begin{array}{l}\text { Interdependence } \\
\text { within the } \\
\text { organisation }\end{array}$ & $\begin{array}{l}\text { 12. Please choose one key team you are an active member of } \\
\text { in this school. What is the name of that team? } \\
\text { 13. Please describe how this team operates and what you have } \\
\text { achieved together recently. } \\
\text { 14. As a team, what sorts of data or information did you use to } \\
\text { judge your progress in achieving these aims? } \\
\text { 15. How has this information influenced the team's work and } \\
\text { what do you hope to achieve together in the future? }\end{array}$ \\
\hline $\begin{array}{l}\text { Theoretical } \\
\text { perspectives }\end{array}$ & $\begin{array}{l}\text { 16. With respect to the previous questions, how would you } \\
\text { summarise your own ways of thinking and reasoning about } \\
\text { your work? } \\
\text { 17. In this school, do you hold conversations with others in } \\
\text { which you openly share views and develop knowledge } \\
\text { about each other's assumptions? }\end{array}$ \\
\hline
\end{tabular}

Each interview was audio taped with the subject's consent. Exhaustive transcripts of each interview were prepared and labelled as draft transcripts. The draft transcripts were sent back to the subjects who annotated them with any deletions, modifications or additions they wished to make to clarify their responses. The edited transcripts were then ready for a final analysis. In qualitative research it is necessary to do data analysis simultaneously with data collection and it is true to say this took place throughout the process of conducting the pilot interviews, preparing draft transcripts, editing the transcripts and conducting the final analysis.

The principals of the participating schools provided copies of documents to illustrate the types of information collected and analysed by their schools for the purposes of planning and evaluation. In the substantive case study, the provided documents provided additional information about the context to which the interviewees referred, so assisted the hermeneutic analysis of interview transcripts.

\subsubsection{Triangulation}

To summarise the methodology of this study, a number of methods were employed so that composite triangulation may be used to ensure coherence in the 
findings. Heuristic methods were used to evaluate the evolution of the research methodology, the planning and evaluation of the case study interview method, the literature review and the development of the theoretical perspective presented in this thesis. Hermeneutic, narrative methods were used for the interpretation of interviews and narrative literature, and a theoretical method, consistent with a naturalistic coherentist epistemology, was used for the evaluation of theories. A combination of heuristic and theoretical methods were used to construct a theoretical perspective that draws on a naturalistic coherentist epistemology, a three-worlds ontology, theory of mind, narrative theory, and distributed cognition theory. 


\section{Chapter 4: Leadership narratives}

This chapter evaluates a number of leadership schemas expressed in narrative. Leadership-as-competence highlights the abilities of the leader; relations between leader and followers are emphasised in leadership-asinfluence; leadership-as-political-action acknowledges the socio-political nature of leadership; the representation of leadership through typologies portrays leadership-as-image; and leadership-as-social relations highlights notions of community, caring, empathy, guidance, and support. In leadership-asperformance, it is what is seen by others that matters; and leadership-asinnovation is the creation of new patterns of social or organisational practice. Finally, despite the popular inclusion of leadership schemas in narrative, some accounts of social and organisational innovation omit or dismiss leadership schemas altogether.

\subsection{Introduction}

What is leadership and where can it be found? How may a leader be identified? Why is it that one person can be singled out as a leader but not another? Situational narratives reveal the common perceptions and understandings of the words, 'leader' and 'leadership'. This chapter examines the meanings attached to these words in a selection of situational narratives, and in doing so seeks to illuminate the cognitive representations underlying these meanings.

Whilst the meaning of a word is an important component of its sense, sense is also determined largely by the context in which it is used. We deal with the variations of sense that arise from a diversity of contexts, by employing schemas of interpretation. The psychological events aroused by these schemas give rise to meaning, as described by Vygotsky:

The sense of a word is the sum of all the psychological events aroused in our consciousness by the word - it is a dynamic, fluid, complex whole, which has several zones of unequal stability. Meaning is only one of the zones of sense, the most stable and precise zone. A word acquires its sense from the context in which it appears; in different contexts, it changes its sense (Vygotsky, 1962, p. 146). 
This chapter presents the variations-of-sense of the words 'leader' and 'leadership' as portrayed or implied in narrative. It is important to note that, since the narrative extracts presented here are drawn from diverse contexts, they are not directly representative of leadership in an educational context. However, it is argued here that these narratives do portray the interpretations that arise from commonly held leadership schemas, and these same schemas are used to interpret educational leadership.

In Chapter 5, this argument for interpretive schemas is explained in terms of folk psychology. The common views of folk psychology, portrayed in situational narrative, also influence the emergence of context-free, theoretical accounts in grand narrative.

\subsection{Schemas of leadership}

Leadership narratives are a window through which we can observe and reinterpret the theoretical perspectives of writers. The following narratives are drawn from a variety of contexts that include, but are not limited to, educational contexts. These narratives are relevant to a study of leadership in schools because they provide a means to understanding the reduction of vicarious experience to the general principles of leadership theory.

Informal, commonsense views of leadership may be understood as the precursors of formal theories. These commonsense views become embedded in culture as they are shared narratively. They also contribute to the ongoing creation and preservation of leadership as a concept, because...

old ideas can be expressed and preserved outside of minds and in the absence of any sustained effort to re-enact or recreate them - for example, in oral narratives ..., in books, drama, music, architecture, and in tools, routines and the organisation of groups (Strauss and Quinn, 1997, p. 115)

The transfer and adaptation of ideas from context to context, via media such as these, contributes to the re-enactment and re-creation of culture. Hence, society 
contributes to the expression and transformation of school culture, whilst schools also contribute to the expression and preservation of the prevailing social culture. In this way that leadership schemas are re-created and preserved.

Leadership schemas are mediated by the mental structures that organise our knowledge. A schema is not a distinct thing but a collection of elements that process information interactively (Strauss and Quinn, 1997, p. 49). Many conceptions of leadership may be understood as social schemas because they are shared by people who have had similar experiences in life, or they are constituted as part of a shared cultural understanding. These schemas do not necessarily function according to the rules of language, nor do they depend on particular words to evoke their use. The new approach to the modelling of schemas employs parallel distributed processing, also known as connectionism (Strauss and Quinn, 1997, p. 50). This view of schemas is much less rigid and much more fluid than earlier uses of the term:

\begin{abstract}
Meanings generated by schemas, in connectionist models, are mental states but are shaped by the learner's specific life experiences and are sensitive to activity in a particular context. While often similar from person to person, context to context, and one period of time to another, they can vary and change (Strauss and Quinn, 1997, p. 50)
\end{abstract}

Hence, the shared understandings of culture are mediated by schemas that share similarities from person to person, but also vary and change according to the experiences of the cultural group. A general concept of leadership seems to be shared by a broad cross-section of social groups in Western culture, and perhaps by other global cultures also. Within Western culture, shared leadership schemas are evident in government, the military, sport and many other forms of organised social action. It is reasonable to expect, therefore, that these shared schemas also influence the understanding of leadership as applied to schools. Whilst the following narratives are not drawn exclusively from school situations, they are relevant to representations of shared leadership schemas and their inclusion in the grand narratives of leadership. 


\subsubsection{The schema of leadership as competence}

The idea that leadership is enacted by people with exceptional skills is a pervasive one. Hence, the grand narrative of leadership-as-competence says people who exhibit extraordinary competence in their fields make good leaders. For example, Ron Barassi ${ }^{51}$ is well known in Australia because of his impressive skills and reputation in Australian Rules Football as a player and as a coach. His frequent calling to the task of rejuvenating failing football teams and his popularity as a motivational speaker, are testimony to his legendary status as a leader.

Leadership-as-competence is a schema that highlights a person's extraordinary practical abilities or technical skills in a given field. Leadership-as-competence may also refer to a person's ability to manage a group of people effectively in a situation requiring cooperative action. For example, the first edition of the Manual of the Victorian Bushwalking and Mountaincraft Leadership Board describes leadership as

...more an art than an exact science... leadership is a relationship that exists between persons in a social situation. Persons who are leaders in one situation may not necessarily be leaders in other situations. It is also recognised that there are wide variations between the characteristics of individuals who become leaders in similar situations, and even greater divergence in leadership behaviour in different situations. The only common factor appears to be that leaders in a particular field need, and tend to possess, superior general and technical competence or knowledge in that field. There is no substitute for personal experience (VBMTAB, 1978, p. 2).

The manual, which was written to assist its readers to 'competently lead walking groups in bush or mountain country in Victoria' (VBMTAB, 1978, p. 2), goes on to suggest a link between the competence of the leader, the confidence and motivation of group members, and the subsequent ability of a group to fulfil its goals. Such a manual would not be written if a strong link between leadership ability and technical competence were not first assumed.

\footnotetext{
${ }^{51}$ For a biography of Ron Barassi, see http://www.ronbarassi.com.au/about/1.htm.
} 
The association made between leadership and competence suggests that a highly competent individual will make a highly able leader; and similarly, that an individual lacking competence will make a poor leader. Ambrose's (1991) history of a company of American soldiers during the Second World War describes leadership in terms of the military competence of officers. One of the stories presented in Ambrose's book describes the defeat of a numerically superior enemy force by troops under the command of company commander, Captain Winters. Winters suggests that if the officers commanding the enemy troops had acted with more military nous, the soldiers under their command may have been able to overwhelm and defeat the Americans:

Later, Winters realised that he and his men had been "very, very lucky." In an analysis, he said the main reason for success was the poor quality of German leadership. The Germans had let the $1^{\text {st }}$ squad get away with sitting in the field waiting for reinforcements. They had allowed two machine guns to pin them down while the three columns of Easy [Company] ran 200 yards across the field in the bayonet charge. They had reacted much too slowly when Winters fired on them from the road. They failed to put together an organised base of fire when the shooting started (Ambrose, 1991, p. 152).

The situation described here hints at a lack of training and ability amongst all ranks, including the enemy officers. In this narrative, the term 'leadership' appears to refer to the competence of all the opposing soldiers in a way that goes beyond the moment to their training, their experience, their ability to act in concert with each other, and the ability of their officers to sum up a situation quickly and make rapid purposeful decisions. Nevertheless, the moment is also important: Winters attributes the success of Easy Company to the luck of the moment. He intimates that, if the German troops had reacted in a much more organised and aggressive way, then his personal interventions may not have been enough to save the day.

As narrators describe the context in which leadership acts occurred, they explicitly or implicitly connect particular leadership acts to those contexts. However, ascribed competence as a leader in one context, or field of action, does not necessarily translate to the recognition of competence in another field. English politician and diarist, Thomas Creevey (1768-1838), observed Arthur Wellesley, the 
Duke of Wellington, as military commander of the allied forces opposing Napoleon in 1815. Here he comments on Wellington's later career as a politician in the English parliamentary system:

Creevey found that in 1822 he held the hero [Wellington] in exactly the same honour as in 1815: honour for his misery at the "loss of lives", honour for admitting his nearness to defeat, honour for the justice done to the enemy, high honour despite the fact that the Duke had since become a politician - "very foolishly" in Creevey's opinion - and done "many wrong and foolish things" (Longford, 1969, p. 490).

Creevey's esteem for Wellington's victory over Napoleon at Waterloo Wellington's military leadership - contrasted strikingly with his low view of Wellington as a political leader. It is apparent in the narrative that Wellington's perceived genius as a military leader was not seen to translate to a similar degree of leadership ability in English parliamentary politics. In this example, a forceful association is made between leadership as technical competence and the context(s) in which it applies.

In educational contexts, people who govern and manage schools may be described as educational leaders. Implicit to this understanding is the view that these people must exhibit a particular kind of competence if schools are to function well under their direction. Callahan (1962) describes how this type of consideration featured in the selection of school superintendents at the time American schools were adopting a business model of school management:

The situation in American education after 1911 demanded leaders who were oriented toward the business side of education, not the social or philosophical side. Products of a business society, they had the qualities and the training (Strayer, Cubberley, and Elliot wrote their doctoral dissertations on financial problems) that enabled them to provide the kind of leadership that a business society required (Callahan, 1962, p. 180).

Callahan reports Edward C. Elliot's view that, for both educational and economic reasons, individuals with 'special technical preparation' were needed in the role of school superintendent. Elliot's reasoning was premised on the rise of technical experts in other areas of American life. He believed that technical expertise was 
needed in education but had been relatively slow in coming. This, he argued, was largely due to the public's belief that special technical expertise was not needed for the management of schools. However, Elliot vigorously asserted his view that a more technically expert approach to school management was needed if the efficiency of the 'public school machine' was to be improved:

The profession and the public had to recognise that "technical skill is not a possession of the multitude but of the few. Power and responsibility must therefore be delegated to the skilled and selected few" (Callahan, 1962, p. 194).

Here the link between competence and context is again made forcefully. According to this narrative, only those with the highest level of technical skill are fit to be leaders - leadership is 'not something that can be done by amateurs'.

Just as narratives about leaders may emphasise their technical competence, counterexamples of leadership assert a lack of competence, as is illustrated by this account of an American officer during an assault on an enemy position during WW II:

[Lieutenant] Dike looked left and could not see his $1^{\text {st }}$ platoon. His other two platoons were moving forward steadily. They were being fired on but had not taken any casualties. But Dike was naked on his left, or so he thought. He made a disastrous decision, the kind of decision that gets men killed. He signalled for the $2^{\text {nd }}$ and $3^{\text {rd }}$ platoons to join Company HQ section behind two haystacks .... First platoon caught up with the company, grouped behind the haystacks. [Lieutenant] Foley came to Dike for orders. Dike didn't know what to do. Foley insisted he had to do something (Ambrose, 1991, p. 208).

In a military context, poor leadership ability 'gets men killed'. Transferring that idea to education, poor leadership 'causes schools to perform badly'. This idea is expressed in the grand narrative that says, 'The difference in leadership makes all the difference. $^{, 52}$

\footnotetext{
${ }^{52}$ Retrieved on 9 December 2006 from the website of Chris Brady at http://www.christopherjamesbrady.com/articles/Results.html.
} 
Poor leadership may be given as a reason for a school's low performance in key performance measures. The Blueprint for Government Schools (2003), released by the Victorian Department of Education late in 2003, is a school improvement initiative based on the premise that high variations exist in outcomes between classes within schools and between schools with similar student populations' (Blueprint for Government Schools, 2003, p. 2). The Blueprint is designed to encourage educational innovation and cooperation between schools and ...

reflects the Government's determination to improve the quality and performance of schools, and to work with schools that have poor student outcomes (Blueprint for Government Schools, 2003, p. 3).

The blueprint contains a number of 'Flagship Strategies'. One of these strategies is titled, 'Building Leadership Capacity'. Much of the detail of this strategy refers to leaders as people holding positions of responsibility (such as principals) and those who may, in future, enter such positions are called 'potential leaders':

We must ensure that the principals and leaders in our schools have the necessary skills and attributes to drive school development and improve student outcomes. To ensure this, the Government will also introduce two initiatives to identify and develop teachers with high leadership potential and to develop the leadership capabilities of existing principals (Blueprint for Government Schools, 2003, p. 20)

In this narrative, the ability to lead is equated by implication with a particular set of skills called 'leadership capabilities'.

Another strategy, titled, 'The Leading Schools Fund', assumes that a school may become a leader of other schools. The stated purpose of the fund is to provide schools with

the incentive and opportunity to reach beyond their current practice and performance' (Blueprint for Government Schools, 2003, p. 27).

The publication also claims that the initiative will facilitate 
strategic partnerships and collaboration between schools so that schools can learn from each other, assist each other and strengthen the government school system (ibid.).

Integral to this initiative is the understanding that leading schools will attain special competence in the delivery of specific education programs and, in turn, lead the introduction of similar approaches in other schools:

Furthermore, secondary schools that are funded for innovative practice will be required to be a teaching school and provide leadership to other schools seeking development (ibid.).

Leadership-as-competence is presented in the Blueprint as the superior knowledge and abilities of an individual person (e.g. a principal) or institution (e.g. a leading school) and the commensurate ability to bring about better educational outcomes.

To possess practical abilities implies that those abilities are practised in situations of need. Narrative accounts will sometimes emphasise the practical abilities of leaders in terms of leadership style or traits; that is, in terms of the characteristics of the individual. At other times, they describe leadership in terms of the particularities of the situation, or entwine the characteristics of the individual with the characteristics of the situation so that their separation in the mind of the reader becomes very difficult indeed. In these cases, the leadership is there - somewhere enmeshed amongst many other abstract ideas, as illustrated by Carlyon (2001). Carlyon comments critically about an historical account, of the role of Sir John Monash in the Gallipoli battle of 6 August 1915, as given by the war correspondent, Charles Bean:

Bean in his official history also damned Monash's leadership on that morning. Monash owned a fine mind and was a good organiser. "But he was not a fighting commander of the type of Walker, M'Cay or Chauvel, and the enterprise in which he was now engaged was one calling for still more - the touch of a Stonewall Jackson, and the recklessness of a J.E.B. Stuart." This misses the point. Monash, as he would later prove in France, was the opposite of Hamilton: better at planning battles than carrying them out in the field. But this had little to do with why his column was stalled. His men couldn't lift their legs; it is not usual for soldiers to want to fall asleep in a strange country with bullets kicking up dust around them. And this first leg to Aghyl Dere was the easier part. These men 
could not have climbed Abdel Rahaman, where the slopes were much steeper than Chunuk Bair, even if Stonewall Jackson was leading them and "Jeb" Stuart had galloped up with a wagon load of scaling ladders (Carlyon, 2001, p. 384).

The way Carlyon describes the situation above suggests that many factors influenced the outcome of battle, least of all leadership. Later in France as corps commander of the Australian forces, Monash was later credited with the capture of the strategically important town of Hamel from the Germans ${ }^{53}$. His leadership successes in France are often attributed to the conceptual abilities he had previously developed in civilian life as a civil engineer. Those abilities included meticulous attention to detail in planning, careful coordination of resources, clarity of communication, and ensuring needed access to information. However, the situation in Gallipoli was vastly different to that of France in terms of Monash's rank and authority, the unforgiving terrain, lack of useful information, poor allocation of resources, inadequate channels of communication, and the apparently inadequate planning by those who were higher in the chain of command.

The schema of leadership-as-competence is one of many employed in situational narrative. Leadership schemas may also be structured according to the influence leaders have on the intentions and beliefs of people.

\subsubsection{The schema of leadership as influence}

Many accounts in literature and film portray leaders as people who are esteemed and whose words and example influence others. Followers may find the leader inspiring because of their trust and respect in his expertise and the sense of purpose they gain from him or her. They readily carry out the wishes of the leader because they believe he or she values them and has their interests at heart. 'Leadership as influence' emphasises the relationship between leader and followers.

\footnotetext{
53 See the Australian War Memorial's online exhibition, '1918 - Australian's in France' for a summary of the battle for Hamel, accessed on 7 December 2006 from the website of the Australian War Memorial at http://www.awm.gov.au/1918/battles/hamel.htm.
} 
The relationship between school leaders and followers, according to Johnson and Johnson (1989, Chapter 1, p. 22) depends principally on leaders. They assert that school leaders should model good practice and lead by example. Doing so, they argue, requires that educational leaders hold clear beliefs about educational practice and be able to speak coherently about their vision and values. In short, they hold to the idea that leaders must be able to inspire others: 'Before most people are willing to perceive someone as a leader, they must believe that he or she is honest, competent, forward-looking, and inspiring' (Johnson \& Johnson, 1989, Chapter 1, p. 8). They describe leadership as the process of winning over the hearts and minds of others, and of encouraging and rewarding good practice - especially when 'the going gets tough'.

Winning over hearts and minds - the idea of leadership as influence - is one that may also be applied to the relationship between teachers and between teachers and their pupils. Frank Tate (1895), at the time an inspector of schools, expressed his view that teachers should be influencers of others:

The test for admission to the pupil teachership should be a severe one, and in Victoria we are gradually making it severer. The first requisite, I think, is that the candidate should give promise of being an influencer of his fellows. There are too many dead-and-alive, unemotional, unenthusiastic pupil teachers in our service now, and we require teachers who have sympathy with their work and with children, who have mental alertness, who have warm blood in their veins, and feel their life in every limb. Only after this first requisite is satisfied should knowledge of methods of teaching and literary ability count (Tate, 1895, pp. 72).

Tate uses colourful language to convey his opinion that the ability to influence others is essential to the work of teachers. It is also clear that Tate believes it is teachers, such as these, who are needed to bring about...

the highest and best results of education - the production of robust, manly boys, who know what they know solidly and reasonably, who can see straight, and feel straight, and act straight (Tate, 1895, p. 144).

Tate clearly conveys a high value on the character of teachers as people who ought to live out and inspire their students to high ideals. 
Although Tate does not use the word 'leadership' in the above accounts, the leadership-as- influence schema is revealed simply by replacing the words 'pupil teacher' with the word 'leaders'. Note here that a schema is a pattern of elements that work together to process information in a particular way. These word substitutions preserve the pattern of influence. In this way, the pattern of inspirational teachership corresponds to a pattern of inspirational leadership.

Tate's portrayal of influential teachership as an indispensable quality for a teacher is therefore, by implication, a call for teachers who possess influential leadership qualities. This is in keeping with the late $19^{\text {th }}$ century authority relationship between teacher and pupils:

A schoolmaster, as his name implies, should be the absolute master of his position and he should be left to please himself as to the best methods to imparting the subjects; interfering with his individualism cramps his energies, prevents him from impressing his mind and force of character on his pupils, makes him and his pupils mechanical, destroys half his power of usefulness (Education: the Defects of the Common School System, 1871, p. 44).

Tate's vision for teachers and their pupils is one that may be interpreted as the living out of high ideals. This is often portrayed as that vital factor that transforms superficial compliance to devotion and willing obedience.

In Guy Sajer's account of the life of a German Soldier on the Russian Front during the Second World War, he presents a personal account of extreme hardship and suffering. Here, he describes the horror and despair experienced in the struggle for survival as the German army retreated before the Soviets during a harsh Russian winter. In the midst of these most unpleasant of experiences, Sajer describes the preciousness of his relationships with those closest to him, including his commanding officer:

Captain Wesreidau often helped us to endure the worst. He was always on good terms with his men, and was never one of those officers who are so impressed by his own rank that they treat ordinary soldiers like valueless pawns to be used without scruple. He stood beside us during countless grey watches, and came into our bunkers to talk with us, and 
make us forget the howling storm outside. I can still see his thin face, faintly lit by a wavering lamp, leaning over, beside one of ours (Sajer, 1999, p. 264).

Sajer builds the reader's understanding of the nature of his relationship with Wesreidau by describing how Wesreidau differed from other officers in the way he treated them:

Our conversations with Captain Wesreidau made a very deep impression on us. His obvious and passionate sincerity affected even the most hesitant, and seemed of another order than the standard appeals to our sense of sacrifice, which left us stupefied and incredulous. He invited questions, which he answered with intelligence and clarity. He spent his time with us, whenever he was free from other duties. We all loved him, and felt we had a true leader, as well as a friend on whom we could count. Herr Hauptmann Wesreidau was a terror to the enemy, and a father to his men. Every time we moved, or were sent out on an operation, his Steiner preceded our vehicles (Sajer, 1999, p. 267).

In this narrative, the influence of the leader arises from the quality of his relationship with his followers - one that is sustained by respect and trust.

Variations of the leadership-as-influence schema may be found in cases where 'inspiration' is replaced with 'fear'. One such example may be found in Major Richard Winter's recollections of Captain Ronald Speirs during the Second World War:

Speirs was very effective. He got the job done. But if you were around and talked to the men who worked under him, he was never liked. Now, he could turn around and walk away and talk to someone at my level and be a completely different guy. He could take orders. He was very likable. (Anderson, 2004)

It seems that Speirs was disliked, but had the fearful respect of those under his command, and was respected by his superior officer because he 'got things done'.

Another variation of the leadership-as-influence schema appears when leadership is portrayed as a process of changing the way people view the world. Callahan (1962, pp.180-8) describes the influence of two men, George D. Strayer and Ellwood P. 
Cubberley, as leaders in educational administration in the United States of America during the early part of the $20^{\text {th }}$ Century. Both men were highly successful in their careers, were about the same age, and both received their PhDs in education at about the same time and from the same institution. In addition, both had written their doctoral dissertations on problems in educational finance. According to Callahan, Strayer's influence was derived principally from his teaching, research direction and the large number of school surveys he directed. Strayer promoted the study of the details of educational administration, with an emphasis on technical, financial and mechanical problems. Cubberley's influence, however, was exerted via his text books, which were widely used throughout the USA. Cubberly wrote mainly about organisational, legal, and financial problems and the history of education. His books frequently referred to business and industry and contained many analogies between those fields and education. Callahan reports that these two men were very influential in shifting the focus of educational administrators from educational philosophy and intellectual pursuits to the values and practices of business.

The Victorian Department of Education also expects that its school principals will influence the institutional culture of schools:

The Government recognises the critical role and responsibilities of principals in improving the educational outcomes of students in their schools. As educational leaders in our communities, principals have a major influence on the capacity of their teachers and the quality of the teaching-learning relationship. Within the system we must provide principals with the support they need to develop a culture of high performance and continuous improvement in schools. By investing in leadership development, the Government will ensure that school leaders are equipped to meet the demands and challenges of their role within the system" (Blueprint for Government Schools, 2003, p. 19).

Implicit in the above statement is the idea that principals need to be supported in their role as influencers and that they must be suitably 'equipped' or trained to use their influence to bring about a certain type of culture within schools - that is, a culture of 'high performance'. 
The schema of leadership-as-influence may be connected to the concept of leadership-as-competence insofar as the motivation to perform competently has cultural significance. This kind of leadership is understood to inspire or coerce others to perform well, even in situations of challenge or hardship. Inspirational leadership may change the view that followers have of their world and alter the focus of their energies so that they perform in a desirable way, which ever way that is conceived. Leadership-as-influence may also be a linked to the political-leadership schema.

\subsubsection{The schema of political-leadership}

Moral issues lie at the heart of the political-leadership schema portrayed in the conflicts of history. Well-known examples of moral struggles include Abraham Lincoln's fight for the abolition of slavery in the USA, Nelson Mandella's efforts to overturn apartheid in South Africa, and Aung San Suu Kyi’s struggle for democracy in Burma.

Aung San Suu Kyi, recipient of a Nobel Peace Prize, is a recognised leader of the democracy movement in Burma. Her name has featured in many news reports, such as in the following example:

Burma's military junta has indicated that it is preparing to free democracy leader Aung San Suu Kyi ahead of resumed talks next month on drafting a new constitution to return the country to civilian rule (Baker, 2004, p. 9).

Suu Kyi has been portrayed by the media as a symbol of quiet strength and nobility in contrast to the raw and ruthless power of the Burmese military junta. The popularity of the democracy movement in Burma is evident in the 1990 landslide victory of Suu Kyi's National League for Democracy in democratic elections - a result that was not recognised by the military junta. Even though the goal of the movement was not achieved at the time, Suu Kyi retained her status as the movement's leader. The larger political goal of democratic government for Burma has not overshadowed the short-term goals of the movement. 
The political-leadership schema may be linked in various ways to the schemas of leadership-as-image and leadership-as-competence. For example, the evolution of democratic processes for hiring and setting the pay of employees, as portrayed by Ricardo Semler, is a complex mix of these three schemas. One of the innovations introduced at Semler's company, Semco, was a biannual evaluation of managers by their subordinates. This involved the anonymous completion of a multiple-choice questionnaire. The questions were designed to measure subordinates' perceptions of the manager's technical ability, competence, leadership and other aspects of the manager's role, and were weighted according to their relative importance. After subordinates completed the questionnaire, their responses were analysed, a percentage grade calculated, and the results publicly posted for all employees to see.

A score of 70 percent was considered the minimum satisfactory outcome of this process. Semler describes the outcome of one particular case of this evaluation process:

\begin{abstract}
We once had a man who ran a large department extremely well, or so we thought. On his first evaluation he got a [score of] 40 [percent]. After looking into it, we concluded that his subordinates were right; he was a great salesman but a terrible leader, and his people were succeeding in spite of him. Solution: we made him the head of a one-person sales staff, and he has shone. With a new leader, his former department did even better (Semler, 1994, pp. 167-8).
\end{abstract}

In this narrative, the competence of the subject (as a salesman) appears to have been judged as strong (presumably because of sales figures). However, his competence as a manager of a large number of subordinates has been judged weak according to a moderately democratic process.

Semler describes how the company proceeded to a stronger form of democratic process by which managers were elected by subordinates. This became an overtly political process, which included 'policy' speeches and debates amongst various interest groups. Semler describes how the atmosphere of the room changed as one of the manager applicants entered to give a pre-election speech: 
As Timoshenko entered the room for his interview, the chatter from the several dozen people who had assembled there ceased. Three different camps sat in different sections of the room. By the rooms were the engineers, including Almir Vieira and Carlos Okamato, the leaders of the opposition. They felt Timoshenko had not only been too tough, but had "micro-managed", which was anathema at Semco. The second group, the division's administrative staff, sat at the far end of the room, at the end of the long mahogany table. Many had had run-ins with Timoshenko and were against him. The last group, the factory supervisors were sitting against the wall opposite the window. They had counted on Timoshenko to solve their bureaucratic problems and seen him turn promises into reality on the shop floor. They were solidly in his camp (Semler, 1994, pp. 172).

Semler goes on to describe how Timoshenko was elected to the position, though not without controversy - and how he subsequently made one of the 'leaders of the opposition', Almir, his deputy. According to Semler, Timoshenko's unit proceeded to become one of Semco's 'great successes'. In this narrative, Semler presents the democratic process as a means of critically appraising a potential manager's leadership ability. Interestingly, he also presents leadership as a changeable political attribute or quality when he describes Almir's move from leader of the 'political opposition' to that of being a leader in support of Timoshenko.

Leadership as politics may be more obviously presented in narratives about the parliamentary systems of Western democracies. However, poignant leadership narratives can also be found in other types of socio-political struggles such as the controversy surrounding Point Nepean. Point Nepean is located at the tip of the Mornington Peninsula, at the head of Port Phillip Bay in Victoria. Once the site of a fortress and a military reservation this area of land became a site of social and political controversy when the Australian Federal Government (the owner of the site) announced its intention to release the land for commercial development. Statements about this site were made in the press by the Federal Government, the Victorian State Government, residents in the local area, environmental groups, representatives of the local indigenous community, commercial developers, and others.

Following the acceptance of a plan, brokered by Melbourne man, John Mickle, to convert the site to a national park and heritage precinct, investment banker, Simon 
McKeon, was appointed by the Australian Federal Government to oversee the process. In March 2004, The Melbourne newspaper, The Age reported that the Point Nepean plan was 'in chaos' following the resignation of two 'key interest groups' who were also backed by Mickle:

The groups said they felt excluded and that communication had broken down. "We all three have a complete lack of confidence in Simon as a leader," Mr Mickle said. "Any questions we have are ignored. Since December after several requests, we had one meeting and the open day is in three days," he said (Fyfe, 2004).

Here the narrative alludes to influential leadership by portraying a leader as one who is trusted by others. However, in this case, the context is also socio-political in nature; the decision to publicise the rift between Mickle and McKeon in a prominent Victorian newspaper clearly has political significance. Causal significance is attributed to the reported lack of confidence in McKeon, insofar as it is given as an explanation for the dissent amongst the interested parties and a reportedly severe breakdown in an alliance of government and non-government parties.

In response to this allegation, Simon McKeon, is reported saying,

I am very confident that at the end of the day the vision will be fulfilled and there is no shortage of demand from other organisations to make the vision a reality," he said. "I couldn't have done more to promote the interests of their organisations. It is absolutely extraordinary (Fyfe, 2004).

In short, McKeon seems to be deflecting the expressed lack of confidence in his leadership by suggesting that he can easily replace the three estranged parties and fulfil the 'vision'. At this point the narrative connects a comment about vision to one about being (or not being) a leader. Here, leadership is portrayed as the fulfilment of vision. To express lack of confidence in a person 'as a leader' (leadership-asinfluence) implies that leadership is the crucial factor at play in this story.

In what ways is political-leadership portrayed in field of education; and is political-leadership a crucial factor in educational practice? Firstly, the politicalleadership schema may be portrayed in the education policies of opposing political 
parties. Secondly, it may be evident in the process of raising support for particular initiatives within the school system and the subsequent resourcing of those initiatives. Thirdly, it may refer to the processes within a school community.

In their book, The Self-Managing School, Caldwell and Spinks (1988, p. 21) stress the importance of a shared culture and vision in the operation of schools. They build on this idea in Leading the Self-Managing School to describe the 'higher order' attributes of leadership. In this view, school leaders have

the capacity to articulate and win commitment to a vision for the school and ensure that vision is institutionalised in the structures, processes and procedures which shape everyday activities (Caldwell \& Spinks, 1992, p. 69).

To 'articulate and win commitment to a vision' is a political process as much as a cultural or organisational one. Leadership-as-political-action may be understood as taking the moral high ground, winning support for a vision, symbolising a social change movement, or as the portrayal of a particular type of personal charisma or image.

\subsubsection{The schema of leadership-as-image}

The schema of leadership-as-image portrays a leader as one who is beheld in a sense of awe and mystery. A leader may be said to have a certain 'look' about him:

Speirs was an officer with a reputation. Slim, fairly tall, dark hair, stern ruggedly handsome, he cultivated the look of a leader, and acted it. One of his fellow D Company junior officers, Lt. Tom Gibson, described him as "a tough, aggressive, brave, and resourceful rifle platoon leader" (Ambrose, 1991, pp. 205-6).

... and a leader may be portrayed as a person who has a reputation for doing extraordinary things:

Standing at the site in 1991 with Winters and Malarkey, Lipton remembered Spier's dash [through broad open fields under enemy fire]. $\mathrm{He}$ also recalled that when they got to the outbuildings of Foy, Speirs wanted to know where I Company was. "So he just kept on running right 
through the German line, came out the other side, conferred with the I Company C.O., and ran back. Damn, that was impressive" (Ambrose, 1991, p. 211).

Particular types of image or reputation may be portrayed as the crucial element of leadership in certain situations. This may be an image of awe and mystery, a particular type of persona, or a reputation for getting things done. Portrayals of particular kinds of image are generally used to distinguish the leader from others within narrative.

Leadership-as-image may be emphasised or deemphasised by the various modes of sense conveyed in language. I had the fortune to make a chance observation of the following interaction between two children during a school event. The two children, a brother and sister, had spent the day with their primary school on an outing to the local beach. One of the activities they participated in was a competition between the four 'houses' of the school, with each house composed of students from every age group. The aim of the competition was for the students in each house to work together to create the longest and most convincing sand serpent possible, within the allocated time limit. As was customary for siblings in this school, this brother and sister were members of the same 'house'. A friendly rivalry was evident between these two. After the competition the brother, a Grade 6 student who was also the House Captain, announced to his sister (with a broad grin on his face) that their house won the competition 'because of my brilliant leadership!' In response, his sister, a Grade 3 student, smiled back in a mildly contemptuous manner and replied, 'Dream on! We won because of us. Everyone did it together!' This interaction had a sense of game about it - evident in the interaction were a sense of competition and a light-hearted 'tug-of-war' with the concept of leadership. More importantly in my view, it presented a situation in which two young children constructed two distinct grand-narratives, each being based on the same situation but portraying the significance of the 'leader' differently.

In similar ways, narrators make choices about whose actions to highlight in narrative and whether or not to assign significance to leaders or leadership. Hence, the narrator will choose whether to portray a particular image of leadership in 
accordance with the purpose of the narrative. Hence, 'leadership as image' is the interpretation of the narrator as much as it is an account of the actions or qualities of a leader.

Leadership-as-image relies on the construction of meaning and the ascription of intentionality by folk psychology. Bruner (1990, p. 13) describes folk psychology as 'a culture's account of what makes human beings tick'. He also says that folk psychology deals with the "nature, causes, and consequences of those intentional states - beliefs, desires, intentions, commitments - that most scientific psychology dismisses in its effort to explain human action from a point of view that is outside objectivity' (ibid, p. 14).

Beliefs, desires, intentions and commitments feature in many of the opinions expressed in the media. For example, leadership may be described in terms of 'an act of principle' or 'rising above your instincts:'

Leadership occurs too rarely in Australia's national life. Occasionally there are glimpses of it in politics. Bob Hawke and Paul Keating remade our national economy by confronting and overturning Labor traditions. John Howard took us into Iraq against a cacophony of opposition but on the presumption that our alliance with the US was paramount. But when was the last time a frontbencher from either side resigned as an act of principle? (Hywood, 2004)

Hywood also comments on leadership in sport when he comments on the actions of the Australian sportsmen, cricketer Adam Gilchrest and swimmer Ian Thorpe. Here, he refers to Thorpe's graceful response to disqualification from selection for competition swimming:

In sport, a crucial measure of our national character, inspired leadership has been sadly lacking. Adam Gilchrist's 'walk' in the World Cup semifinal was an exception... So it was uplifting this week to see 21-year-old swimmer, Ian Thorpe show all those who seek the limelight, in any field, what leadership is all about... Now Thorpe has set the benchmark for grace in adversity. He has moved from being a great sportsman with nice manners into a genuine leader... Ian Thorpe has shown much older men that real leadership is rising above your instincts (ibid.). 
Australia's highly competitive performance in Olympic swimming events, together with the high participation rate of Australian children in swimming as a sport ensures a high profile for swimming sports amongst the Australian public. However, in this account, Hywood presents leadership as the public portrayal of moral fortitude in a way that transcends context and, therefore, in the form of grandnarrative. Here the leadership-as-image schema is employed by the narrator together with other schemas. These schemas are value-laden.

The schema of leadership-as-image is employed by assigning value to images. When Carolyn, Acting Assistant Principal of Eucalypt School, was asked what it was that sustained her in her role, she said,

\begin{abstract}
Often, when I'm confronted with issues and think, "How am I going to react or deal with this situation?" I think of my mentor, she was a leading teacher here, and is now principal at another school, and who is just fantastic. So, I guess I do often think of her: How would she react? How would she handle this situation? (Interview B7).
\end{abstract}

Here, the image that Carolyn constructed about her 'fantastic' mentor was helpful to her as she built up her experience in the role of Assistant Principal.

The schema of leadership-as-image employs the symbolic interpretation of first-hand observations, second-hand reports or a culturally constructed reputation. Consequently, leadership as image is conveyed in accordance with the narrator's interpretation of events, her personal view of leadership, the purposes of her narrative, and the constraints that culture places on her.

\title{
4.2.5 The schema of leadership-as-social-relations
}

The social nature of leadership arises from the emotional and cultural nature of social interaction:

As so much of leadership is dependent upon emotional interaction between people, attitude of mind becomes very important; particularly the attitude of the leader towards the party members. If you, as leader, have a selfish attitude, then you are undertaking this course for the wrong 
reasons, and if that attitude persists you will not become a first class leader (VBMTAB, 1978, p. 2).

The above account stresses the importance of the attitude of the leader toward others and attaches causal significance to such attitude. Leadership may entail dictatorship or facilitation. However, a balanced view may also be advocated: 'Clearly it is not enough to be a facilitator, enabling others to pursue their own goals. Nor is a dictatorial approach adequate; effective leaders hold both of these aspects in tension' (Kaldor et al., 1997, p. 159). Hence, the leadership-as-social-action schema requires that harmony be established and sustained between leader and followers so that organisational goals may be fulfilled.

A counterexample of that type of balance is evident in the following extracts from Ambrose's account of the WW II members of Easy Company, $101^{\text {st }}$ Airborne Division. Here hostility is portrayed between leader and followers:

There was talk about who was going to shoot [Captain] Sobel when the company got into combat. Tipper thought it was just talk, but "on the other hand I was aware of a couple of guys in Company E who said little but who in my judgement were fully capable of killing Sobel if they got the chance." (Ambrose, 1991, p. 37).

Ambrose reports that Easy Company's level of dissatisfaction and sense of estrangement from Captain Sobel, their first commanding officer, became so intense that Sobel was transferred from the Company to another command. The noncommissioned officers (NCOs) of the company who were brought to a high level of competence under Sobel's ruthless training regime were not confident about Sobel's decision-making ability on the battlefield. Paradoxically, Ambrose (1991, p. 210) says Sobel was responsible for soured relations with his NCOs, but that he also played an important part in shaping the NCOs' exceptional ability as soldiers and leaders. It seems that Sobel's callousness drove the NCOs and the men closer to each other. This intimate comradeship, in turn, enabled the NCOs to sustain the men's morale and keep the company together despite the extreme trials of battle. Leadership-as-social-image is conveyed through descriptions of attitudes and intentions as much as the description of a situation. 
Direct and indirect, intentional and unintentional means of communication feature in causal accounts of leadership as social action:

\begin{abstract}
We can see that feelings and/or emotions are very important in our communications with others. But don't forget that your own feelings are very closely associated with your attitude. A selfish attitude tends to express itself in impatience and a lack of understanding or concern for the feelings of others, which can very quickly lead to party management problems. A selfless attitude leads to a sincere desire to guide others so that they will themselves develop and achieve fulfilment (VBMTAB, 1978, p. 3)
\end{abstract}

The importance of intention and attitude is also evident in the decisions made by Bob Hawke, Prime Minister of Australia from 1983 to 1991. Hawke deliberately gave up drinking alcohol from the time he became head of the parliamentary Labor party to the time he was deposed in a ballot of the Caucus in December 1991. In his memoirs, he said:

\begin{abstract}
... I was conscious of two things. First, I often behaved badly in drink ... Too often excessive drinking led me to do and say things of which I was ashamed ... Second, I had no illusions about the magnitude of the challenge ahead. As I strove for leadership for the party, I would be under constant scrutiny and any alcohol-induced lapse of behaviour would be headline news. Above everything else, however, I believed that if I was now going to become a leader of my country, it was entitled to expect from me a 100 per cent commitment of my mental and physical energy. I did not believe I could give that if I continued to drink (Hawke, 1994, pp. 103-4).
\end{abstract}

Hawke was very much aware of the importance of good social relations in parliamentary politics and government and took action to prevent those relations becoming soured (leadership-as-social-relations). He gave up alcohol so that he could be seen as performing at his best in the role of Prime Minister (leadership-ascompetence). He also saw this action as necessary in preserving the image of Prime Minister as responsible citizen and as committed and competent leader (leadershipas-image). Hawke also did very well politically in the Australian electorate, being reelected repeatedly to become one of the longest serving Australian Prime Ministers (leadership-as-politics). Additionally, Hawke's reputation for genuineness of 
intention and attitude was fostered by his strikingly emotional public performances in the media.

\subsubsection{The schema of leadership-as-performance}

The portrayal of emotion appears to be an important element of the leadership-asperformance schema. Shakespeare reveals the intimate intentions and feelings of Brutus as he proceeds towards the murder and overthrow of the dictator, Julius Caesar:

Since Cassius first did whet me against Caesar, I have not slept.

Between the acting of a dreadful thing

And the first motion, all the interim is

Like a phantasma or a hideous dream

(Shakespeare, Julius Caesar, Brutus in Act II, Scene I, 60).

Shakespeare's portrayal of conspiracy, treachery, and political intrigue in Julius Caesar portrays the dark and foreboding events of a leadership contest. Such contests may be found in many other examples in history.

England's Queen Elizabeth I had her share of troubles both before and during her reign. In his biography of Elizabeth, Starkey (2000) describes Elizabeth's leadership style as a combination of dramatic performance and the purposeful communication (or concealment) of thoughts and feelings. Starkey partly shares the view of one of Elizabeth's earlier biographers, Victorian Bishop Mandell Creighton, that Elizabeth's teenage simplicity was artificial - the acting of a part. Starkey portrays Elizabeth as a skilful writer, director and actress in the unfolding events of political intrigue of $16^{\text {th }}$ Century England. In Starkey's view, this predilection to performance is one that Elizabeth shared with others:

Elizabeth's style was not unique to her. Instead, it was typical of almost all the leaders of intellectual and political opinion in the brief reign of Edward VI. Power politicians such as Seymour, Somerset and John Dudley, Earl of Warwick, and intellectuals such as Thomas Smith, William Cecil and John Cheke, who made the leap from Cambridge to the court, were progressives and thought of themselves as such. Their progressivism, embraced every field of activity: like Elizabeth, they were 
Protestants in religion, classicists in art and amateurs of what C.S. Lewis pejoratively called the "drab" style in literature... Elizabeth, less constrained than the Archbishop, was a leader of the movement and her household was one of its principal academies (Starkey, 2000, p. 87).

How did Elizabeth attain the art of performance, so skilfully enacted in her troubled rise to the English throne? She received instruction in the most advanced curriculum of the day from very able tutors, but it was 'her lessons in the school of life that mattered more' (Starkey, 2000, p. ix). Elizabeth undertook an apprenticeship in royal life - she learned distrust, double-dealing and 'a swirling obfuscation of language in which the more she said the less her meaning was clear' (Starkey, 2000, p. xi). Elizabeth's experiences so shaped her view of the world that she saw it necessary to construct a particular sort of character in the latter years of her reign bewigged, face caked in carmine and white lead, invoking a curious combination of fear and love in those with whom she dealt.

Leadership as drama is the central theme of Starrett's $(1990,1993)$ publications: The Drama of Schooling and The Drama of Leadership. Starratt (1993) speaks of drama both as metaphor and as a homology of social life. According to Starratt (1993, pp.117-130), schools share with society the elements of drama: expressiveness through communicative action, conventions of communication framed by the status of communicators, culturally grounded gender and class roles, cultural symbols and artefacts, and the dialectical interaction of the individual and society. Starratt unpacks the roles of educational leaders through the metaphors of player, director, stage manager, critic, playwright, and drama coach. Above all, the leader is a player:

Leaders must be players within the drama. The cast needs to have a sense of the leader's involvement in the action. Leaders have to feel the anxiety of not knowing how things will work out, the excitement of the action the ambiguities and conflicts in relationships, the struggle to meet deadlines and the satisfaction of a completed performance (Starratt, 1993, p. 138).

According to Starrett, only as players can leaders recognise that their personal integrity is at stake as the values and purposes of their organisations are played out. 
Integrity, however, may be a matter for re-interpretation according to Masters (2004) in his article, What makes a good leader. Masters emphasises links between principal leadership and school performance when he interprets research findings to say that:

Leaders in schools achieving outstanding results also demonstrate a willingness to take risks, to experiment with new approaches and to support colleagues proposing new initiatives and changes. These leaders push the boundaries of administrative and systemic constraints and, when necessary, bend rules to achieve what they want...Leaders in schools achieving outstanding results create a climate of success and high expectations. They take every opportunity to "talk up" the school" (Masters, 2004, p. 12) [italics added].

In this grand narrative, Masters describes what he considers the essential traits of school principals. Implicit in this account is a particular theoretical view of leaders in schools.

Grand narratives of this sort may also be inserted into situational narratives. Reflecting on 'effective school research', Holden's (2004, p. 21) narrative account reports Ken Rowe's view that the traditional emphasis on the principal as the key determinant of school effectiveness is flawed. In Holden's account, Rowe is reported as saying that the traditional view of principals, as the predominant influence on school performance, completely misses the point. Rather, the primacy of leadershipas-performance (where leadership equates to the actions of the principal) is challenged by the idea that teachers, not principals, are the key determinants of school performance. Holden, a journalist, presents a situated narrative about an academic's grand narrative. Here, the grand narrative is that the link between schools and teachers is mediated by teachers, not by principals.

Leadership-as-performance is played out in the drama of life. School principals, and others at the interface of school and public, become the focus of attention in the same way that the director and leading actors of a motion picture become the signifying symbols of the performance in the eyes of the audience. The effectiveness of an organisation (however, that is judged) may be attributed to particular individuals (leaders) or, holistically to the collective performance of the members of 
an organisation. However, the common view tends to attribute significant performances or innovations to those in a formal position of authority or reputation.

\subsubsection{The schema of leadership-as-innovation}

Acts of innovation are also attributed symbolically to leaders. The personal computing (PC) business is a business of constant and rapid innovation. In his history of the evolution of the personal computer (PC), Cringley (1996, p. 16) says, 'In the PC business, constant change is the only norm, and adolescent energy is the source of that change.' Adolescent energy may have driven the PC innovation, but that energy does not necessarily translate to organisational leadership. Cringley explains his view of where one should not look for leadership in the PC industry:

Good programmers don't usually make good business leaders. Programmers are typically introverted, have awkward social skills, and often aren't very good about paying their own bills, much less fighting to close deals and get customers to pay up. This ability to be good at one thing and so bad at another stems mainly, I think from the fact that programming is an individual sport, where the best work is done, more often than not, just to prove that it can be done rather than to meet any corporate goal (Cringley, 1996, pp. 103-4).

According to Cringley, leadership means refined social skills and strong financial management ability. However, he also suggests that ego gratification can play a part:

I wish, sometimes, that I could say this personal computer stuff is just a matter of hard-headed business, but that would in no way account for the phenomenon of Steve Jobs. Co-founder of Apple Computer and founder of NeXT Inc., Jobs has literally forced the personal computer industry to follow his direction for fifteen years, a direction based not on business or intellectual principles but on a combination of technical vision and ego gratification in which both business and technical acumen played only small parts (Cringley, 1996, p. 182).

Possession of a grand vision is also highlighted in Cringley's narrative when he compares Bill Gates with Henry Ford:

Gates and Ford are similar types. Technically gifted, self-centred, and eccentric, they were both slightly ahead of their times and took advantage of that fact. Ford was working on standardisation, mass production, and 
interchangeable parts back when most car buyers were still wealthy enthusiasts, roads were unpaved, and automobiles were generally built by hand. Gates was vowing to put "a computer on every desk and in every home running Microsoft software" when there were fewer than a hundred microcomputers in the world. Each man consciously worked to create an industry out of something that sure looked like a hobby to everyone else (Cringley, 1996, p. 99).

Steve Jobs and Bill Gates are often typecast as the key innovators in the PC revolution but this view fails to acknowledge their dependence on the ideas and technological innovations of others. For example, the graphical user interface now common in computer operating systems arose from the creation of bitmap technology and the 'mouse' by XeroxPARC (Cringley, 1996, pp. 80-2) and the rapid evolution of computer hardware arose from the production of the first 'PC' from offthe-shelf components by IBM (Cringley, 1996, pp. 119-158).

In schools, when the innovative role of individuals is recognised, such recognition necessarily shifts the focus from principals to teachers. Traditional leadership narratives portray principals as the people who set direction and make key decisions that others follow. In some narratives, principals are cast as the people who make up for 'an absence of leadership by everyday teachers' (Fullan, 1993, p. 75). However, the principal of Eucalypt Primary School presents a different schema of innovation:

What my aim is - is to continually improve the quality of teaching and learning in the school. I say that as a two-sided thing because unless teachers are involved in continual improvement through professional development, through self-reflection, through working with each other, then I don't believe that student improvement will happen. Again, I can say that student achievement has increased dramatically here in the last three years because I think teachers have picked up on computer education, that we've had a stronger focus on literacy, teachers have been looking at the way they teach it, how children learn. They see all of it as being responsible, as a total group, for the progress of the children at school. So, I think that is the focus of Education Planning Committee as well, that continual improvement of both teachers and children [Interview B5]. 
In this narrative, innovation is attributed to a partnership between teachers and those in formal leadership roles. This notion of interdependent action by principals and teachers gives rise to the idea of leaderless-innovation.

\subsection{An absence of leadership schemas}

Sometimes, narratives describe innovation independently of any leadership schema. Two examples, one from industry the other from science, are given here.

Chandler (1969) gives an account of the innovation of a decentralised corporate structure developed independently by four major US companies. Chandler describes the complex interactions that led to the innovation of new corporate structures: interactions of the market, availability of resources, population growth, technological development, company growth and changes in company strategy:

\footnotetext{
Four phases or chapters can be discerned in the history of the large American industrial enterprise: the initial expansion and accumulation of resources; the rationalisation of the use of resources; the expansion into new markets and lines to ensure the continuing full use of resources; and finally the development of a new structure to make possible continuing effective mobilisation of resources to meet both short-term market demands and long-term market trends (Chandler, 1969, p. 385).
}

Chandler's account of the structural changes in four major US companies is comprehensive. However, though he acknowledges the importance of administrators (executives and managers) in this process, he does not describe them as leaders or their work as leadership. Rather, his account emphasises an interactive learning process by which 'organisation builders could learn from others' (Chandler, 1969, p. 17). Here, Chandler is referring to the four companies following accepted practices in American industry. However, as they proceeded beyond existing practices towards an innovative multidivisional structure, 'others learned from them' (ibid). In this account, structural innovation arose from a two-way, interactive process.

A similar interactive process is evident in scientific innovations. Albert Einstein's relativity theories dramatically changed the theoretical structure of physics. Yet 
White and Gribben (1993) in their biography of Einstein do not refer to Einstein as a leader, or his work as leadership. However, they do include Moszkowski's vivid description of the idolisation of Einstein and his theory by the public:

\begin{abstract}
Newspapers entered on a chase for contributors who could furnish them with short and long, technical or non-technical, notices about Einstein's theory. In all nooks and corners, social evenings of instructions sprang up, and wandering universities appeared with errant professors that led people out of the three-dimensional misery of daily life into the more hospitable Elysian fields of four-dimensionality. Women lost sight of domestic worries and discussed coordinate systems, the principle of simultaneity, and negatively charged electrons. All contemporary questions had gained a fixed centre from which threads could be spun to each. Relativity had become the sovereign password (A. Moszkowski, quoted in White \& Gribben, 1993, p. 145)
\end{abstract}

Einstein was very influential in the worldwide scientific community, achieved notoriety amongst the public (at certain times and places), was awarded a Nobel Prize, and was asked (but declined) to accept the post of President of Israel. Einstein played an important role in the innovation of theoretical physics - innovation that in time led to technological innovations. Einstein's response to the Israeli government regarding the Israeli Presidency is reported to include the following paragraph:

\begin{abstract}
All my life I have dealt with objective matters, hence I lack both the natural aptitude and the experience to deal properly with people and to exercise official functions. For these reasons alone I should be unsuited to fulfil the duties of that high office, even if advancing age was not making increasing inroads on my strength (White \& Gribben, 1993, p. 245).
\end{abstract}

Einstein acknowledged Chaim Weizmann, first president of the modern nation of Israel, as the person who 'bore the heavy burden of leading us towards political independence' (ibid), but viewed himself as lacking the experience and personality to perform presidential duties. Here, Einstein is equating leadership with the ability to 'deal properly with people;' that is, to deal with the subjective matters of social action rather than the objective matters of theoretical physics. Despite his public popularity and the substantial influence he had on the progress of science, he did not consider himself suitable for a formal leadership position. Hence, a schema of socialinfluence need not be a leadership schema. 


\subsection{Conclusion}

The various nuances of leadership portrayed in narrative may arise from its connection to concepts such as competence, influence, political action, image, social relations, performance, and innovation. Are these connections necessary or contingent qualities of leadership? Attributions of leadership appear to be time and context dependent, where context is created from particular features of history, geography, culture and technology. A leadership schema is a web of belief by which particular patterns are recognised in given situations. In Chapter 5, the distinction between situational narrative and grand narrative is examined. 


\title{
Chapter 5: Narrative meaning
}

\begin{abstract}
In this chapter, situational narrative is distinguished from grand narrative. The richness of situational narrative is tempered by the ambiguity of propositional attitudes and the problem of interpreting non-literal language, imagery, and symbolism. Situational narrative pertains to the psychological world, whereas grand narrative pertains to the theoretical world. If propositional attitudes cannot be eliminated from psychological explanations, then the translation of the psychological to the theoretical will not be fully attainable. This presents a problem for research methods that seek to construct formal leadership theories from narratively expressed accounts.
\end{abstract}

\subsection{Introduction}

What is it that distinguishes situational narrative from grand narrative and how is this distinction relevant to leadership theory? Contemporary leadership theory relies heavily on empirical evidence obtained through questionnaires and interviews. This kind of research obtains data from subjects who are unavoidably connected to the social milieus of their present and past. Each social milieu possesses its own history one that is intertwined with the histories of other milieus. The memories and perceptions that subjects have about leadership cannot be separated from those histories, simply because they constitute the temporal and spatial realities of their lives.

In organisational settings, Argyris (1961, p. 327) concluded from his research that it was impossible for people to separate leadership from the other organisational factors and still speak of reality. A complete account of the historical and social influences on the thoughts and actions of people is seldom if ever possible because the realities they perceive are composites of inextricably connected variables. When leadership theory is derived from situational narrative, it abstracts away from the detail of context-specific instances. Such inferred generalisations present the problems of inductive reasoning and testimony. They are also susceptible to 'information cascades', by which false ideas are propagated and magnified as they 
are passed narratively from person to person; and to the risk of fallibility that arises from the ambiguity of propositional attitudes (Vickers, 2006; Adler, 2006).

In a broad sense, research subjects are situated in the history of Homo sapiens. They make persistent use of 'common sense' thinking. This common way of explaining and predicting the actions of people constitutes the rules of thumb, or informal theories, of 'folk psychology'. Folk psychology pervades explanations of human and social action and is very difficult to eliminate from responses to research questions. No matter what method is used - case study, laboratory technique, statistical method, or any other method - research questions activate the socially mediated interpretations of subjects and researchers. When eliciting explanations about people, both the questions of researchers and the responses of subjects, tend to employ the propositional attitudes of belief, desire and intention. The propositional attitudes pervade situational narrative.

\subsection{From situational narrative to grand narrative}

When situational narrative takes the form of story it locates events in space and time and arranges those events according to plots or themes. In doing so, they locate characters within a particular cultural and historical milieu and describe their actions in terms of intentions and beliefs. Whilst situational narrative conveys a contextspecific story, grand narrative presents theoretical principles that stand independently of time or situation. A single text may contain situational narrative or grand narrative, or both forms of narrative arranged in sequence or in parallel.

The Biblical parable of the sower ${ }^{54}$ is an example of a sequential arrangement of situational narrative and grand narrative. In this case, the situational narrative comprises a story about a farmer sowing his seed with the seed landing on various surfaces: a path, rocky places, among thorns, and in good soil. The story is then interpreted by means of a grand narrative of general principles, with each principle corresponding to one of the four surfaces mentioned in the situational narrative.

\footnotetext{
${ }^{54}$ Matthew 13, Mark 4, Luke 8
} 
A parallel arrangement of situational and grand narrative is presented in Justin Gaader's book, Sophie's World. This book is a story about the events shared by two fictional characters: a teenage girl, named Sophie Amundsen, and her mysterious mentor, Alberto Knox. In the story, Gaader blends a situational narrative with a grand narrative of philosophy. The text includes a signature characteristic of the two classes of narrative: the propositional attitudes of situational narrative, and the theoretical propositions of grand narrative, with each standing alongside the other:

\begin{abstract}
Now Sophie looked at the envelope she had been given. It was addressed "To Hilde." She opened it and read aloud: "Dear Hilde. If the human brain was simple enough for us to understand, we would still be so stupid that we couldn't understand it. Love Dad." Alberto nodded. "True enough. I believe Kant said something to that effect. We cannot expect to understand what we are. Maybe we can comprehend a flower or an insect, but we can never comprehend ourselves. Even less can we expect to comprehend the universe (Gaader, 1996, p. 276).
\end{abstract}

In this text, the sentence, "I believe Kant said something to that effect," presents a propositional attitude, whilst the sentence, "We can never comprehend ourselves," expresses a theoretical proposition. Propositional attitude sentences employ intensional verbs (such as, for example, 'believes' or 'hopes') and take the form 'A believes that B'.

Some leadership commentators believe that storytelling is of itself an important skill for people who exercise leadership. Denning (2005) sets out to demonstrate the similarity between leadership and story telling, as performance arts. His argument is that since leadership and story telling both entail the communication of ideals, leaders should develop the art of story telling. The storytelling he has in mind fulfils a number of purposes: to illustrate a point; to act as a springboard to a complex idea; or to act as a means of sharing knowledge in the form of problems, solutions and explanations. This requires that some connection be made between the storyteller and the audience, preferably by capturing their interest in some way. Denning suggests that eight patterns of narrative can be utilised for leadership purposes. In Table 5.1 below, Denning's patterns have been aligned with the leadership schemas that were introduced in Chapter 4. The purpose of the table is to demonstrate that the 
categories proposed by theorists can be selectively generalised from cognitive schemas.

Table 5.1 Alignment of narrative purpose with leadership schemas

\begin{tabular}{|l|l|}
\hline $\begin{array}{l}\text { Leadership schemas } \\
\text { (Chapter 4) }\end{array}$ & $\begin{array}{l}\text { Patterns of narrative purpose } \\
\text { (Denning, 2005) }\end{array}$ \\
\hline Leadership as influence & Motivate others to action \\
\hline Leadership as image & $\begin{array}{l}\text { Build a sense of trust and } \\
\text { acquaintance with the leader }\end{array}$ \\
\hline Leadership as performance & $\begin{array}{l}\text { Convey a particular set of } \\
\text { organisational values }\end{array}$ \\
\hline Political leadership & Build trust in the organisation \\
\hline Leadership as social relations & $\begin{array}{l}\text { Get others working together; } \\
\text { neutralise gossip and rumour }\end{array}$ \\
\hline Leadership as competence & Transmit knowledge \\
\hline Leadership as innovation & $\begin{array}{l}\text { Communicate the leader's vision } \\
\text { for the organisation }\end{array}$ \\
\hline
\end{tabular}

The associations made in the above table arise from the following: To motivate others is to influence their behaviour. Apart from coercive power relations, this requires that the 'follower' perceive the 'leader' from a standpoint of trust. This in turn requires that the follower be sufficiently acquainted with the leader. Although rhetoric may transmit values, the follower's experience of the leader gives credibility to those values. Hence, experience takes on the characteristics of performance. Building trust in the organisation requires that it be positioned within socio-political settings and therefore requires political action. Similarly, cooperation requires the right sort of interpersonal relations. Finally, perceptions of competence are accompanied by the expectation that knowledge will be declared and enacted, and innovation is commonly perceived to be an outcome of deliberate intention (though not of necessity).

Dennings' patterns of narrative purpose can be drawn from leadership schemas as generalisations. The schemas employed in narrative are socially mediated patterns of 
thought; and their socially mediated nature aligns them with categories of folk psychology. As categories of common thought, schemas constitute a form of narrative knowing.

\subsection{Narrative knowing}

The narrative way of knowing, according to Gudmundsdottir (1991), is necessary for an understanding of the world of human beings. Narrative knowing constitutes an indispensable means for the communication of understanding. This narrative way contrasts with the paradigmatic way or search for universal truth conditions, also known as grand narrative (Clandinin and Connelly, 2000, pp. 29-33), or technical rationality (Schön, 1983, pp. 68-9).

Gudmundsdottir describes narrative construction as a heuristic process that unifies theories, themes and specific events into larger units. Narrative construction requires skill, judgement and experience; and is the form of knowledge employed by practitioners in case histories, narrative explanations, and packets of situated knowledge. Narrative thinking matches a problem, situation or idea to selected stories and schemas. It also gives cohesion to shared beliefs by organising facts and events to make them comprehensible.

Narratives constitute a whole within which events connect and interact. As such, they may be understood to be occupying a three dimensional space consisting of a selective dimension, a combinative dimension and a schemata dimension (Gudmundsdottir, 1991; Hawkes, 1977; Jakobson, 1956) (See figure 5.1 below): 


\section{Figure 5.1 Three dimensions of narrative}

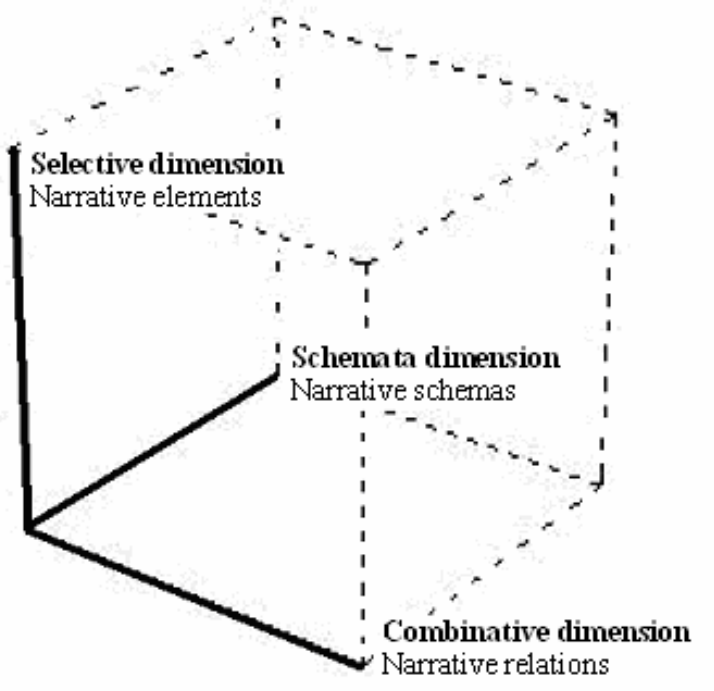

The selective dimension comprises the shorter stories, examples, illustrations and explanations of narrative, selected by the author, and functioning as the building blocks of the narrative. These narrative elements are connected in a way that gives coherence to the story. It is in the combinative dimension that relationships between narrative elements are declared. These relationships give continuity and structure to the narrative and require that the reader comprehend both the narrative elements and the declared relationships. That task is facilitated by the schemata dimension, which comprises a selection of schemas. These culturally familiar patterns enable the reader to make sense of the various narrative elements and the relationships between them. Schemas are mental structures and expectations that mediate the specific instances of the narrative and the general expectations of the reader. Variations of schema theory are applied to the study of narrative and cognitive science:

The essence of schema theory in the cognitive sciences is that in large measure information processing is mediated by learned or innate mental structures that organise related pieces of our knowledge (Strauss and Quinn, 1997, p. 49).

Schemas provide a selection of categories and patterns for the classification of experiences. They shape the meanings we impart to present experiences and enable us to reinterpret past events. Schemas enable us to make decisions quickly so that we 
can get things done in the present. They also guide our expectations for the future and enable us to imagine future possibilities. Many schemas are interpersonal or cultural in nature, since they comprise shared or relational understandings. These cultural schemas enable people to fill in missing information, therefore making social interaction and communication possible.

Leadership schemas of the sort introduced in Chapter 4 convey particular patterns of social interaction. In describing her role, Sarah the principal of Eucalypt Primary School describes her main responsibility as 'educational leadership':

I think the main purpose of my role is educational leadership in the school. Provision of educational opportunities for children would be number one. I'm [also] Executive Officer of School Council obviously. There is obviously the management role of the financial aspects of the school and the organisational aspects of the school. I also see communication with the school community as a prime role that I have in the school, communication with the wider community and beyond (internationally as well). Student welfare is a major role, it's a specific responsibility of mine, but it is also a role that I see as a very important role for principals to have. So, student and staff welfare, personnel issues ... Educational leadership, I suppose, is the one we would like to be in full control of (Interview B5).

Leadership is joined to two other concepts in this account. Sarah's conception of educational leadership seems to include educational provision and communication with others. She also distinguishes educational leadership from other activities including routine management and organisation tasks, welfare and personnel issues, and day-to-day communication. Underlying each of the words, objects and events depicted in this narrative are schemas that constitute the socially mediated interpretation of experience. The leadership schema employed by Sarah in this case incorporates some concepts but excludes others. The terms and sentences Sarah used in the interview also carry particular meanings relevant to the moment of the interview and the interpretive framework she brought to the interview based on her experiences.

According to Clandinin and Connelly (2000, p. 19-33), educational experience should be studied as situational narrative because experience happens narratively. 
The temporality and rich 'seeming' of experience stands apart from the theoretical generalities of grand narrative. That which is said about a person or situation obtains meaning within a larger context, as time and circumstances change, meanings can change. Tensions between situational narrative and grand-narrative arise because the former is sensitive to time and situation whilst the latter is not. Narrative thinking is distinct from other forms of thought and cannot be translated to grand narrative without also employing theoretical filters. The trouble with theoretical filters is they seek 'capitalised truth' by interrogating stories as data rather than seeking understanding and verisimilitude. The result is a contrivance that abstracts away from the credibility of vicarious experience to cold, hard, distant 'facts', (Davies, 1998, pp. 481-483). Successful narrative thinking requires thinking at the boundary of narrative thinking and other forms of inquiry rather than subsuming narrative into formal theoretical categories:

Table 5.2 Tensions of situational and grand narrative

\begin{tabular}{|l|l|l|}
\hline $\begin{array}{l}\text { Tensional } \\
\text { category }\end{array}$ & Situational Narrative & Grand Narrative \\
\hline Temporality & Time dependent & Independent of time \\
\hline People & Focused on people & Essentially people-free \\
\hline Action & $\begin{array}{l}\text { Action is a narrative marker } \\
\text { with attributed historical } \\
\text { significance }\end{array}$ & $\begin{array}{l}\text { Action is direct evidence with } \\
\text { attributed causal } \text { significance }\end{array}$ \\
\hline Certainty & Interpretations are contingent & Interpretations are necessary \\
\hline Context & Situation-dependent & Independent of situation \\
\hline
\end{tabular}

Clandinin and Connelly associate grand-narrative with Schön's (1983, pp. 68-69) technical rationalism. The distinction between rational-reductionist thinking and narrative thinking corresponds to the distinction between technical-rationalism and Schön's reflection-in-action. Reflection-in-action, as applied to a practical context does not depend on the categories of established theory and technique, but on the construction of a new theory of the unique case. Here, thinking and doing (or ends 
and means) are not kept separate as they are in technical rationality; they are redefined interactively so that reflection-in-action can proceed, even in situations of uncertainty or uniqueness.

Despite replicating many familiar patterns, the dynamics of school practice also create situations of uncertainty or uniqueness. Both narrative thinking and reflectionin-action centre around the telling of story:

Schön makes it possible for many of us to tell the story of teacher education (and other professional education) in a way that runs counter to the technical teacher education we are encouraged to sponsor and study, and he makes it possible for the story to legitimate our professional memory of professional practice (Clandinin \& Connelly, 2000, p. 36).

Paradoxically, the retelling of story is made possible by the reduction of the rich and multi-faceted nature of experience to that which can be written in a book or promulgated as conventions of professional practice. Presented in this way, observations or experiences have little bearing on the claims made by researchers or practitioners because they are interpreted through whatever social structure, ideology, theory or framework is at work in the inquiry. Consequently, narrative inquiry is viewed with suspicion by formalists because it entails the reconstruction of experience and context.

Narrative inquiry views people as the embodiments of lived stories, something that is not possible in formalist inquiry. Although, as Edwards (1997, p. 286) points out, all narratives 'deploy generalised analytic categories such as types of persons, actions, events, and dispositions'. Nevertheless, narrative inquiry cannot legitimately view people as exemplars of formal ideas, theories or social categories (such as race, class, gender, power, and so on). In presenting vicarious experience to others, narrative reduces the particularities of a situation to a form that can be easily communicated. Hence, the narrator interprets the 'lived story', consciously or unconsciously through the lens of particular ideas, theories and social categories. 
The following example, drawn from a case in perioperative nursing illustrates the translation of a situational narrative to grand narrative. The first example, a situational narrative, is an extract from a nursing student's journal entry:

The surgeon stared at me with eyes that could have pierced glaciers, no warmth and no smile in them ... maybe he was concentrating ... The surgery begins. I look at the surgeon, the scout, my trolley. I need confirmation. I watch the surgeon's eyes watching me, I stare back, he raises his eyebrows, not in a puzzled manner but in a comforting way ... Ah ... I'm relieved ... I must be performing OK (Gartner, Latham and Merritt, 1996).

In this account, the student nurse imbues her observations with meaning. Her interpretations ('I need confirmation', 'I'm relieved') take on social, relational and emotional qualities.

After sharing the journal entry with peers and reflecting on the discussion it stimulated, the student prepared a subsequent journal entry. The next example, an extract from that entry, reduces the vicarious and particular to a general principle:

In thinking about the meaning of my story ... The eyes of theatre are powerful. They communicate emotion and hide no truths. I thought about how many times eyes, without verbal communication have been instructive for me ... Perioperative nurses should never underestimate the power of their gaze, especially in a world behind masks (Gartner, Latham and Merritt, 1996).

This second example takes the form of grand narrative. Whilst both types of narrative employ the interpretation of observation; in the first example, the interpretations are spontaneous and intuitive; in the second, they are reflective and theoretical. The subject ('perioperative nurses') and predicate ('never underestimate ...') constitute a theoretical proposition; and the word 'should' is an indicator of value. Hence, the second narrative is formally theoretical.

\subsection{Schemas as organisers of knowledge}

When constructing a narrative account, the narrator selects items from memory according to the schemas in use. Each schema constitutes a pattern, which can 
accommodate some items and not others. Those items that fit the pattern are selected whilst those that do not are ignored. When a schema is partially filled out, it is enacted as if all the items required for the pattern are present. In this way, meaning is apportioned to the items by reference to the schema.

The use of existing schemas can be both a help and a hindrance to memory:

Schemas can both help memory by permitting reconstruction of partially forgotten material and disrupt memory by making people think they remember something which they have actually falsely inferred from a schema (Hill, 1981, p. 210)

Predetermined associations and meanings can be applied to phenomena that match an existing schema, thus removing the need for lengthy deliberation. Schemas enable economical use of cognitive resources. Once enough items have been recalled to enact part of the schema, the entire schema can be activated without the need to recall further items. This simply requires that sufficient items be sampled so that other candidate schemas can be ruled out. However, when a situation requires the recall of unrelated elements, for which no schema is available, the original phenomenon is altered or replaced so that it can fit an existing schema (Hill, 1981, pp. 207-8). Items that do not fit an existing schema are simply discarded to enable an existing schema to be partially enacted. The partial enactment of the schema is sufficient to activate the entire schema as if all the components of the schema were present in memory the first place. False inference, therefore, arises when phenomena cannot be accommodated in existing schemas; that is, when the required schemas are absent.

Examples of absent schemas can be found amongst people who are unfamiliar with new technologies. In cases of this sort, a combination of conceptual naivety and lack of a fitting vocabulary can result in a dysfunctional response from the user. Consider the story of the computer user who, upon finding he could not successfully log into a local network, frankly informed the technical support person that, 'I am entering the password just like you and everybody else does - I type in five stars!' (End User, 2007, p. 28). A new computer user, one who possesses a schema that 
directly links keystrokes to the characters appearing on a computer monitor, could feasibly presume that the characters appearing on the monitor are in fact the characters being typed. However, in this case, the absence of the appropriate cognitive schema prevents the user from correctly attending to the characters being typed on the keyboard.

Another story has been told of the user who informed the technical support person, by telephone, that he could receive emails but was not able to send them. The technical support person decided to try a test with his own email address. When he read out the address over the telephone, he heard the user enter a key stroke in excess of the number of letters in the address. It turned out that the user had been typing 'at' instead of '@', hence entering an invalid email address (End User, 2007, pp. 28-29). In this story, the user lacked a schema for the entry of '@' in an email address, so instead applied a phonetic spelling schema to the utterances of the support person.

The ability to learn the structure of a language is separate from the ability to understand the meaning of an utterance. Hence, it is possible to recognise or even generate a grammatically correct sentence, whilst also lacking a suitable understanding of its meaning. Whilst sentences are generally more meaningful than words, the meaningfulness of an utterance depends on the possession of an appropriate schema. This requires familiarity with the content of the sentence (perhaps due to experience); and whether the type of organisation used in the utterance is simple and apparently logical, or complex and apparently illogical (Hill, 1981, pp. 188, 197-198). Familiarity with such modes of organisation is gained through social interaction.

\subsection{Narrative structure}

The creation of narrative does not occur solely through the processing of information by the individual. Bruner (1990, pp. 99-138) describes how a narrative way of expressing self has emerged with the 'cognitive revolution' and the idea of situated action in cultural psychology. Developments in computational neuroscience have translated the concept of learning and action to the concept of information 
processing. The idea of situated action emerged from the understanding that people construct social realities that are both negotiated with others and distributed across culture and environment (Bruner, 1990, p. 105). According to this view, a person's knowledge is stretched interactively across brain, body, artefacts, culture and environment. Hence, knowledge is not isolated to one's head, but distributed across

\begin{abstract}
the notes one has put into accessible notebooks, in the books with underlined passages on one's shelves, in the handbooks one has learned how to consult, in the information sources one has learned how to consult, in the information sources one has hitched up to the computer, in the friends one can call up to get a reference or a "steer", and so on almost endlessly (Bruner, 1990, p. 106).
\end{abstract}

Socially mediated histories are created and expressed through the narrative expression of social life. These histories recursively shape the cultures through which self is expressed. As self and others are rendered narratively, it becomes possible to ask, 'Why one story and not another?' In this way, a predominant schema can be altered or replaced so that a new perspective is adopted. The narrative construction of meaning mediates cultural interaction and gives rise to a distributed view of self.

Historical narrative may be interpreted in three ways (Callinicos, 1995, p. 46-47). In the first interpretation, a narrative history captures both the participants' subjective experiences and the way events are objectively interconnected. In the second, the effect of narrative on the reader is given greater significance. In the third, narrative is seen as the sole genre of historical writing. This third alternative requires the concept of emplotment (muthos), developed by Aristotle in the Poetics as the core structure of narrative (Callinicos, 1995, pp. 47-52). Emplotment, he says, allows us to understand the central function of narrative in expressing the temporality of human experience.

Emplotment must be understood as a 'poetic act whose critical moment comes when the writer seeks a personal understanding of the events of which she intends to give an account' (Callinicos, 1995, p. 49). To do this, the writer must prefigure the entire set of events reported in the source data. This prefigurative act can match the 
four tropes of figurative language: Metaphor, Metonymy, Synecdoche, and Irony ${ }^{55}$, to four 'modes of emplotment': Romance, Tragedy, Comedy and Satire ${ }^{56}$. These modes provide clues to the meaning of a story by revealing the kind of story that has been told (See Table 5.3). This narrative organisation of historical writing does not involve simple technical decisions; nor is it imposed by the nature of the writer's source data. Rather, the narrative possesses theoretical content, whether real or imaginary, prior to it being actualised in any speech or writing.

Table 5.3 The correspondence of tropes of and modes of emplotment

\begin{tabular}{|c|c|}
\hline Tropes of Figurative Language & Modes of Emplotment \\
\hline Metaphor & Romance \\
\hline Metonymy & Tragedy \\
\hline Synecdoche & Comedy \\
\hline Irony & Satire \\
\hline
\end{tabular}

Since content choices must be made, as the writer chooses a particular mode of emplotment, narrative takes on a character that reflects the ontological and epistemic choices made by the writer. Hence, narrative accounts involve the selection of events and the imposition of a structure on those events. Callinicos argues that these choices are not dictated by the available empirical evidence but by the beliefs and purposes of the writer; and that 'historical narratives, by virtue of their tropological organisation, always fail in their attempt to represent reality' (Callinicos, 1995, p.

\footnotetext{
${ }^{55}$ Metaphor depicts things in terms of their similarities and differences; metonymy substitutes a part of a thing for the whole; synecdoche represents a presumed quality of the whole through a part of the whole; and irony figuratively negates that which is affirmed literally.

${ }^{56} \mathrm{~A}$ romance is a tale depicting events in a way that appeals to the imagination of the reader; a tragedy presents events in a serious or sombre way; comedy presents events in a cheerful or humorous way; and satire exposes and denounces the vices, abuses or follies evident in a course of events.
} 
52). One might wonder whether Callinicos is using the trope of hyperbole in this statement. Nevertheless, this is a view that recognises the limitations placed on narrative as a representation of reality.

To Carter (2003), the world speaks a multitude of symbolically expressed stories that say much more than can be comprehended. Here, narrative is the medium through which temporal events are expressed both semiotically and linguistically with events being expressed through the manipulation of 'arrays' and 'arrangements'. Here, the term 'array' refers to a kind of temporal division (e.g. minutes, years, etc.) whilst 'arrangement' signifies particular selections and combinations of arrays. These arrangements are semiotically constituted as relations 'between dates, relations between dates and events, and relations between events' (Carter, 2003, p. 20). It follows that the rhythmic nature of experience, itself poetically ordered, gives rise to a beginning, middle and end. Experience, constitutes a symbolically enacted story by which the features of life stand alongside the features normally associated with story. In this view, reality and representation are simply two levels of the one semiotic system.

Narratives group events in certain, theoretically disposed ways. The justification of a particular narrative rendition of past events lies in its coherence - its internal consistency and its relationship to other narratives. Historical stories may also assist subjects to form coherent conceptions of themselves and their relations with others. Marchitello (1997, p. 94) describes narrative as a form of possession to which 'the other' always exists secondarily. In this view, narrative is dedicated to the eradication of difference and the ideological production of sameness. Even anecdotes, which are often offered as ideological and politically neutral representations of historical fact, cannot be freed from the predispositions of those who report them:

Faith in the presupposition of meaning is under-written - indeed, it is produced - by the a priori projection of a narrative that is by definition totalising and teleological (Marchitello, 1997, p. 123). 
Marchitello illustrates this tendency to bias by tracing discourse about Sir Thomas Browne. Beginning with the writings of Browne, Marchitello identifies what he calls artefaction in the practices of seventeenth century natural science. He traces these processes within pseudo-medical science, first in nineteenth century phrenology, then in early twentieth century racial craniology. Via a number of fortuitous events these two 'narrational practices' adopt Browne's disinterred skull as the object of their inquiries. Marchitello argues that both phrenology and racial craniology 'posit the immediacy of the skull and the immanence of human identity within it' (Marchitello, 1997, p. 8) - and that both forms of pseudo-science contribute to the production of a narrative of racism.

The one skull becomes the subject of widely differing accounts regarding its significance; Marchitello presents extracts from four newspaper articles, published in 1840, that give conflicting accounts of Browne's remains. He summarises the four accounts (described in chronological order of publication) as follows:

The first narrative depicts a perfectly preserved body; the second, a typically decomposed body, but one whose signs (e.g.' the "finest conformation" of the skull and the "beautifully developed" forehead) seem to guarantee something like a formerly perfect body; and the third and fourth describe the decomposed body and, with special attention and focus, the unusually shaped head. (Marchitello, 1997, p. 141)

Taken together, the four accounts illustrate a shift from the understanding of Browne's remains as a collection of essentially anonymous and meaningless objects to that of an historical artefact infused with meaning and identity. In a similar way, Hamlet attributes significance to the skull portrayed in Shakespeare's play only after it has been identified as the skull of Yorick. It is the identity of the skull, together with Hamlet's affection for Yorick, that gives meaning to the skull; Yorick had been absent for a long time and was much missed. According to Marchitello (1997, pp. 128-129), the epistemological claim attributed to this material object arises from a desire to 'fend off incoherence'. In keeping with the narrative production of meaning, the narratives of Browne's skull underwent periods of establishment, revision and change. 
Marchitello asserts that the narrational portrayal of 'any act, any utterance, any textual or cultural practice' (Marchitello, 1997, p. 188) presents a theoretically constrained version of truth and nothing more. In our daily lives, we find satisfying theories, theories that make up our worlds as we see them and enable us to fashion dreams and ambitions. Nevertheless, some narratively expressed 'truths' may have adverse or even destructive social consequences. The narrative of Mein Kampf is an example of the destructive kind.

According to Shirer (1960), Hitler writes of his desire for Germany to become a 'folkish' state. What Hitler has in mind here is a state of racial purity - one that encapsulates his distorted view of the 'beauty and dignity of higher mankind' (Shirer, 1960, p. 89). Not only, he says, must a folkish state be based on race but it must also embrace all Germans and 'the aristocratic idea of nature' (Shirer, 1960, p. 89). This view could not accommodate democracy. In its place, Hitler proposes the Führerprinzip (leadership principle) by which every leader has authority downward and every follower has a consequential responsibility upward. This, Hitler believed, would give rise to an elite breed of leaders:

This principle - absolute responsibility unconditionally combined with absolute authority - will gradually breed an elite of leaders such as today, in this era of irresponsible parliamentarianism, is utterly inconceivable (Hitler, 1943, p. 450 - as quoted in Shirer, 1960, p. 90).

The seed sown by ideas such as this gave rise to many horrors. Leadership of this sort, conceived as absolute authority, and seeking absolute power, not only gives support to despotism, it fails to acknowledge the realities of social life.

To propose leadership as the solution to socio-political or organisational problems is to risk distraction from that which can make a difference. No individual, leader or otherwise, exists independently of others and of history. The social forces that serve to ameliorate radical changes in the status quo are distributed across humankind, the artefacts and technologies we create, and the planet we inhabit. No one individual could ever control such delocalised power. However, ideas that are distributed across a social network can have a powerful influence. 
The leadership principle, as Hitler perceived it, arose from Germany's turbulent history, including the decimation of the German people during the Thirty Years War; the barbaric serfdom imposed on the German people after the 1648 Peace of Westphalia, and the later military successes of the Prussian army. The result was a socio-political setting that favoured the acceptance of the leadership principle amongst the German populace:

Germany never recovered from this setback, Acceptance of autocracy, of blind obedience to the petty tyrants who ruled as princes, became ingrained in the German mind (Shirer, 1960, p. 92).

Hence, the history that had shaped German culture enabled Hitler to institutionalise the leadership principle and adopt the title, 'Führer (leader) of the German Reich' (Shirer, 1960, pp. 226-227).

Whilst the rhetoric of autocracy and blind obedience is unlikely to occur in contemporary narratives of school improvement, the general rhetoric of the 'power' of leadership persists in Western democracies. A cacophony of differing leadership principles are put forward as the solution to all kinds of organisational problems in Western society, including the organisational problems of schools. This rhetoric is somewhat diluted and muddied by the plethora of competing models of leadership and the differing 'labels applied to the same conceptual terrain' (Harris and Muijs, 2005 , pp. 1-7). However, principals and teachers continue to describe their day-today work in terms of propositional attitudes.

\subsection{Propositional attitudes}

The propositional attitudes are evident in both factual and fictional accounts of events. In her novel, The Secret Garden, first published in 1910, Francis Burnett (1956) portrays the remarkable change that comes upon the central character of the story, Mary Lennox. She explains this transformation according to the way Mary and the other characters think about the world: 
One of the new things people began to find out in the last century was that thoughts - just mere thoughts - are as powerful as electric batteries as good for one as sunlight is, or as bad for one as poison. To let a sad thought or a bad one get into your mind is as dangerous as letting a scarlet fever germ get into your body. If you let it stay there after it has got in you may never get over it as long as you live.' (Burnett, 1956, p. 236)

According to this depiction, thoughts have the same power as physical forces; that is, 'disagreeable thoughts' and 'sour opinions' affect the physical well-being of the person in a detrimental way, whilst having a mind filled with wonderful sights, smells and interests has the opposite effect. In this story, thoughts, which are objects of mind, are given significance in the domain of the physical so that mind affects body.

The connection of mind and body, or brain and body, is one that is sometimes given weight in scientific investigations, and sometimes not. For example, the research conducted by Thomas de Marse (University of Florida Scientist: 'Brain' in a Dish Acts as Autopilot, Living Computer, Oct 21, 2004) portrays brain as nothing more than a computation device. De Marse is reported to have grown rat neurons from embryonic tissue and trained them to 'fly' a simulated aircraft. This is purportedly a case of a parallel distributed processing system (rat neurons) controlling a serial sentence processing system (a computer program). Another research program points to evidence of the brain being the central source of fatigue, rather than the body (Randerson, 2004). These two examples require that brain and body be envisaged as distinctly separate entities. However, this is not a view shared by all researchers.

To Damasio (1994), brain and body are united; they do not exist as separate entities. This view is supported by the way brain and body are indissociably integrated by mutually targeted biochemical and neural circuits' (Damasio, 1994, p. 87). Damasio (1994, pp. 93-96) favours the view that mind emerges from the concerted action of various 'parcellated' systems - systems that are centred on, but not localised to, specific regions of neural activity in the brain. He suggests that thought is simply the manipulation of topographical images, created as neural 
representations. In this view, the images that arise from the microstructural representations of neuron circuits are manipulated according to various facts and strategies that are either innate, or acquired through experience. Damasio (1994, p. 94) believes this repository of facts and strategies is stored as 'dispositional representations'. These dispositions are also associated with certain emotional responses based on prior experience.

In folk psychology, the dispositions and values of thought are expressed by propositional attitudes. These are the expressions of belief, desires, fears, and so on, that pervade story-telling and common-thought. The propositional attitudes offer reasons for why people act in the way they do, such as in the following example from The Secret Garden:

\begin{abstract}
Martha's face became red with fright. "Eh! Miss Mary!" she said half crying. "Tha' shouldn't have done it - tha' shouldn't! Tha'll get me in trouble. I never told thee nothin' about him - but tha'll get me in trouble. I shall lose my place and what'll Mother do!"
\end{abstract}

"You won't lose your place," said Mary. "He was glad I came. We talked and talked and he said he was glad I came" (Burnett, 1956, p. 115).

In the context of the story, the sentence, 'Tha'll get me in trouble', expresses the maid's alarmed belief that the housekeeper of the manor would hold her accountable for Mary's conversation with the master's son. The sentence can be legitimately paraphrased to read, 'Martha believes that Mary's discovery will get her into trouble with the housekeeper'. Hence, the sentence is of the form 'A believes B', which fits the general form of a propositional attitude sentence.

In The Secret Garden, the housekeeper can be viewed as the formal leader of the household because she has authority over the servants and is in charge of the manor when the master is absent. Hence, the situation portrayed here can be interpreted as a description of a leader-follower relationship. The emotional aspect of this relationship is depicted in the emotional content of the narrative - Martha's face becoming red with fright, and her half-crying, are physiological indicators of emotion. That Mary possesses the propositional attitude of fear is supported by the 
sentences she utters; the implied weight of those sentences as conveyed by the exclamation marks; and the physiological phenomena presented in the text. It seems that all propositional attitudes, either overtly or covertly contain some degree of emotional content. If this is true in all cases, then the leadership narratives that employ propositional attitude sentences must also have emotional content.

\subsubsection{The distinction between theoretical propositions and propositional attitudes}

In many cases, leadership theories formalise or objectify the subjective content of leadership narratives. However, theoretical propositions differ in significant ways from propositional attitudes. A propositional attitude is the disposition held by a subject towards a proposition. For example, the statement, 'John believes that effective leaders provide direction to stakeholders,' is an example of a propositional attitude. It is important to note that the sentence expresses, but does not in itself constitute the propositional attitude; the sentence declares an attitude held by the subject. Sentences of this kind are distinguished by the presence of intentional words such as 'believes,' 'wants,' 'likes,' and 'hopes.' They are sometimes extended to animals as in, 'my dog wants to go for a walk,' or to plants or non-living things (in an anthropomorphic sense) in statements such as 'my hydrangeas like lots of water,' or 'glass has a water-loving surface.' In the latter two cases, however, the propositional attitudes are expressed non-literally.

To the Roman statesman and philosopher, Lucius Annaeus Seneca (4B.C.A.D.65), propositional attitudes carry ethical weight that theoretical propositions can never carry. Seneca, himself a writer of tragedies and of moral, literary and scientific essays, writes a scathing criticism of the writings other philosophers, who he claims lack moral character and purpose. Seneca criticised any pursuit of knowledge that fails to inculcate virtue; with respect to literary scholarship, he draws a distinction between theoretical propositions (analysis, accounts, and principles) and the propositional attitudes (fear, desire, passion): 
Which of these paves the way to virtue? Attentiveness to words, analysis of syllables, accounts of myths, laying down the principles of prosody? What is there in all this that dispels fear, roots out desire or reins in passion? (Seneca, Letter LXXXVIII, 2004, p. 151).

Importantly, what Seneca conveys in his writing is that propositional attitudes have emotional content. Here, Seneca extends his criticism to musical scholarship and geometry and speaks of being 'upset' or of 'smiling':

Turning to the musical scholar, I say this. You teach me how bass and treble harmonise, or how strings producing different notes can give rise to concord. I would rather you brought about some harmony in my mind and got my thoughts into tune... The geometrician teaches me how to work out the size of my estates - rather than how to work out how much a man needs in order to have enough. What use is the ability to measure out a portion of an acre with an accuracy extending even to the bits which elude the measuring rod if I' $m$ upset when some high-handed neighbour encroaches slightly on my property? The geometrician teaches me how I may avoid losing any fraction of my estates, but what I really want to learn is how to lose the lot and still keep smiling (Seneca, Letter LXXXVIII, 2004, p. 152).

What Seneca seems to be saying here is that theoretical knowledge is less important than the right kind of propositional attitudes. This is in keeping with a folk psychological view of the world. However, whilst propositional attitudes predominate over theoretical propositions in folk-psychology, materialism eschews propositional attitudes in favour of theoretical propositions. The radical claim of strong eliminative materialism is that propositional attitudes can play no part in the explanation of human cognition (Churchland, P. M., 1981).

\subsubsection{The intensional content of propositional attitudes}

The radical claim of strong eliminative materialism says 'that our ordinary, common-sense understanding of the mind is deeply wrong and that some or all of the mental states posited by common-sense do not actually exist' (Ramsey, 2003). The motivation for this hypothesis arises from the view that psychological processes are nothing more than physical processes of the human brain (Churchland and Sejnowski, 1992, p. 1). The rapid progress being made in computational neuroscience appears to add weight to this view, with the further claim that the 
theoretical framework resulting from a co-evolution of neuroscience and psychology is bound to be superior to folk psychology (Churchland, P. S., 1986). If folk psychology is simply a common-sense theory of the world, and that theory turns out to be false, then propositional attitudes lose credibility as explanations of the world. Yet, formal leadership theories rely on the interpretation of propositional attitudes in survey and interview data. As was shown in the previous chapter, common-sense views of leadership also make extensive use of propositional attitudes.

In the following interview extract, Margaret, a 'leading teacher' of Eucalypt Primary School, explains her work in guiding and assisting a team member, Eric, in the use of a computer for planning purposes:

We've done all our major planning for this term together, which was good... so that he has a very good idea of what to do; it makes him feel a lot better. Also, I'm working with him on the computer literacy because he hasn't used computers before. We're trying to do a lot of our planning on the computer with him, so he can see [how the computer can be used]. I've got him now taking the laptop home and using the laptop, he learned how to use it last week, it was good (Interview B8).

This statement and the surrounding paragraphs in the interview transcript (See Appendix 5) employ several propositional attitudes. They include the intention to plan the classroom program with the team member so that he knows what is to come, the belief that his taking the laptop home will help him to improve his skills on the computer, and his desire to feel more comfortable with his work. If strong eliminative materialism is a true account of the world, then it would seem that Margaret's account would need to be pared down to eliminate the propositional attitudes, simply because (it is assumed) they cannot express true states of the world. This would simply leave us with nothing more than the various physical states that are realised as one person interacts with another. Even the concept of planning would be eliminated because that implies purpose and purpose is an intentional proposition.

Propositional attitude sentences express objects of mind. These are the objects of folk-psychology or common sense through which human behaviour is explained (Lycan, 2005, pp. 197-8). They qualify as statements of belief, simply because they 
are attitudes to propositions about the world. The intensional content of propositional attitudes attributes beliefs to self or others to explain their behaviour ${ }^{57}$. The attitude expressed in a proposition also requires that it be founded in experience; otherwise, the attitude could not exist independently of the proposition.

Chalmers (1996, p. 20) posits two concepts of belief: a deflationary concept and an inflationary concept. There must be at least a deflationary concept of belief that is purely psychological and devoid of conscious experience; that is, the right psychological state will resemble belief in many important ways. The deflationary concept also allows for some sort of relation to the environment. In contrast, the inflationary concept of belief requires conscious experience as the necessary condition of a true believer. What Chalmers is suggesting here is that psychological properties are the minimum conditions for belief and that no feature of belief can go beyond the phenomenal and psychological.

According to Chalmers (1996, p. 22), the instantiation of a phenomenal property results in the instantiation of a corresponding psychological property. Whilst a phenomenal property gives rise to conscious experience, the corresponding psychological property arises from cognitive processing. The tendency to conflate the phenomenal with the psychological arises because our language for phenomenal qualities is derived from our non-phenomenal language. There is very little that can be said, for example, about greenness apart from saying that it is green. Hence, there is a temptation to dismiss the phenomenal as being identical with the psychological, simply because this makes the problem of explaining conscious experience much less troublesome.

\footnotetext{
57 'Intension' (with an 's') should not be confused with 'intention' (with a ' $t$ '); intension is the sense of a word, whereas an intention is a purpose or goal. The sense of a word corresponds closely to its intension; it is intensions, rather than definitions that are the primary determinants of meaning (Chalmers, 1996, p.78). The intension and extension of an expression approximates to the sense of the expression and the situation to which it refers. This sense/reference distinction also corresponds to the distinction between the connotation and denotation of the expression (Bullock, Stallybrass and Trombley, 1988, p.165).
} 
Chalmers (1996, pp. 24-25) argues that the mind-body problem can be readily divided into two parts: an easy part and a hard part. Whilst the psychological aspects of mind pose many puzzles and technical difficulties, they do not present deep metaphysical problems. However, the phenomenal aspects of mind and the corresponding nature of consciousness still pose deep metaphysical mysteries. Whilst cognitive science has succeeded in explaining psychological properties according to physical properties, Chalmers (1996, p. 25) suggests that a residual problem remains: How do we explain the relationship between the psychological and phenomenal aspects of mind? A view that employs a combination of phenomenal and psychological concepts allows belief description to sometimes be more phenomenal than psychological, sometimes more psychological than phenomenal (Chalmers, 1996, pp. 16-22). The phenomenal aspect of a belief arising from sensation, for example, is much more prominent than its psychological aspect; and the psychological aspect of learning tends to be more prominent than its phenomenal aspect.

Belief is often considered a psychological property (Chalmers, 1996, p. 19), so that to believe something is true is to behave in a way that would be appropriate if it were true. Hence, the attribution of belief to a person can be employed as a means of explaining that person's behaviour. Nevertheless, this conception of belief seems to be incomplete, as Cresswell (1985, p. 129) points out when he says, 'whether a person (or other entity capable of having propositional attitudes) is or is not in such a state may well depend on features external to the person'. Chalmers (1996, pp. 2021) seems to agree with this view when he suggests that, aside from the phenomenal and psychological aspects of belief, certain beliefs could also depend on the state of the environment.

\subsubsection{The ambiguity of propositional attitudes}

In most cases, the intensions associated with most high-level concepts can be analysed according to their structure and function (Chalmers, 1996, p. 79). An examination of the structure of sentences shows that significant structural and logical 
differences exist between propositional attitudes and theoretical propositions (Cresswell, 1985, p. 4). Consider the following two sentences:

(1) Henry will pass the exam.

(2) Everyone who does not sit the exam, or fails, will have done something Henry will not have done.

In any given situation, sentences (1) and (2) necessarily have the same meaning. They express the same theoretical proposition so that in every situation where (1) is true, (2) is also true, or in every situation where (1) is false, (2) is also false.

However, the next two sentences do not have the same meaning, because it is possible for them to express two different propositional attitudes.

(3) Mary believes that Henry will pass the exam.

(4) Mary believes that everyone who does not sit the exam, or fails, will have done something Henry will not have done.

It is possible to imagine a situation in which (3) is true whilst (4) is not. For example, it may have not occurred to Mary to think of the situation in the way described in (2), or she may not have realised that sentence (2) will be true in all those situations where (1) is true.

The difference between theoretical propositions and propositional attitudes, according to Creswell (1985), lies in the intensional states attached to words like 'believes'; these words are sensitive to the propositions expressed by the separate parts of a sentence as well as to the sentence as a whole. Propositional attitude sentences present the problem of ambiguous structure; choosing one structure over another will change the meaning of the sentence. Consequently, propositional attitudes are semantically ambiguous. 
Creswell's (1985, pp. 17-23) solution to the structural ambiguity of propositional attitude reports is to take a propositional attitude to be a de re report. A de re report simply exploits the ambiguity of the sentence such that the object of belief, rather than the word 'belief' is ambiguous ${ }^{58}$. Creswell's analysis aims to show that the problem of the meaning of propositional attitudes is not a problem of belief, nor of representation, but of 'possible worlds'. In this view, a theoretical proposition is taken to be true in all those worlds in which it is true.

The sense of an expression consists, roughly, of the meanings of the parts of that expression combined in a structure that reflects the structure of the sentence (Cresswell, 1985, p. 77).

The upshot of Creswell's analysis is that 'meanings cannot be representations of any sort' (Creswell, 1985, p. 53) and, if his analysis is correct, semantics cannot be reduced to psychology or cognitive science.

Whilst theoretical propositions do not possess structural ambiguity, they can be sensitive to the situations to which they are applied. A given theoretical proposition may be true in some situations and false in others. The propositional attitudes, however, possess ambiguous structure regardless of the situations to which they refer, and are vulnerable to fallacy due to that structure. Walton (1996) shows that ambiguity in sense can lead to errors in reasoning, such as the fallacies of equivocation, amphiboly, accent, and form of expression. The problem of equivocation occurs when the same word is used in two differing senses within an argument; amphiboly arises from ambiguity in the grammatical structure; accent refers to a verbal stress, innuendo or written emphasis that gives rise to contrary meanings; and form of expression ${ }^{59}$ is due to shifts of meaning by grammatical inflection. The problem posed by fallacy is that

\footnotetext{
${ }^{58}$ There are three known interpretations of de re belief, which McKay and Nelson (2006) label as the 'syntactic', 'semantic' and 'metaphysical' interpretations. The interpretation taken by Creswell (1985), is a semantic interpretation.

${ }^{59}$ The fallacy of form of expression (after Aristotle) is also known as the fallacy of figure of speech; though this latter term should not to be confused with the more general use of figurative language.
} 
it was supposed to be an argument, normatively speaking, but failed to meet this normative requirement. The normative requirement put in place in this conception of fallacy is contextual. It relates to what is or is not an appropriate type of move in a given type of dialogue that two parties, by convention or mutual consent, are supposed to be engaging with each other (Walton, 1996, p. 269, italics in original)

Convention and mutual assent are products of social interaction. Whether a given text qualifies as an argument or not is determined both by the text and by the social milieu in which it is read. The reader may re-write the text so that, which is considered central to the reader's concerns and interests, is brought to the foreground and that, which is unwelcome, is relegated to the background (Adams, 1986, pp. 104105). However, the reading of narrative is a psychological process. The semiotic system of the text presents psychological intentions to the reader in the form of socially acceptable selections and situations. Hence, both the text itself, and the framework brought to the text by the reader contribute to the understanding of the text. This is also the case in researchers' analysis of narrative.

In his case study of leadership in a New Zealand primary school, Brooker (2006, pp. 61-63) reports that three theoretical propositions 'emerged' from the gathered research data. The methods used in his study included semi-structured interviews, by which subjects responded to open-ended questions; concept-map interviews, by which subjects physically constructed concept maps whilst giving an account of their thinking and decisions; and the researcher's narratively written personal reflections. Although there were other important elements in his methodology, the study relied substantially on narrative accounts. Theoretical propositions were derived from the propositional attitudes of the narrative by a process of differentiation and reduction.

Nevertheless, the validity of the theoretical propositions derived from the interviews may be questioned. Whilst these propositions might be valid when applied to an individual situation, they could well be invalid or inadequate to the task of explaining other situations. The structural ambiguity of propositional attitude sentences poses deep problems for researchers who attempt to generalise from those sentences. To derive theoretical propositions from such sentences the researcher 
depends significantly on his or her preconceptions and assumptions to 'fill in' absent information.

Narrative explanations of organisational behaviour also employ propositional attitudes. This is evident in the explanations given by those in formal leadership positions in schools. The following example is an account given by school principal, Sarah, when asked about her personal achievements and her work in the school:

I'm a very verbal person. I think that is why I became a leader. The things that caused me to have leadership qualities are the things that I have to, at times, hold back on as the leader of the school, such as, chairing meetings for example. I very rarely chair a meeting, I never chair a meeting in front of the staff (they think I'm appalling). I am a person that is all over the place, goes off track, so I've had to quieten down and listen, use a lot more of my ears than my mouth. I've changed, I've attempted to become far more aware and listen to people more than I was (Interview B5).

The beliefs expressed in this account can be re-stated as follows:

- I believe that leadership qualities arise from actions such as speaking out and chairing meetings

- I believe that speaking out and chairing meetings are contingent attributes of leadership, they do not apply to all situations.

- I believe that the acts of listening and being aware are qualities of leadership.

Another example comes from an interview with a teacher. Responding to a question about shared values and beliefs within the school community, Leading Teacher, Margaret, said:

Yes, I think they do [have shared values and beliefs]. Even though they might have differences, I think we are in a very... Arty...gifted [local] community in that way. They are very, very supportive of the school and what the school is doing (Interview B8).

The propositional attitudes expressed in this account can be re-stated as follows:

- I believe that the school community possesses shared values and beliefs. 
- I believe that those values and beliefs are those typical to an artistically minded community

- I believe that the local community agrees with what the school is doing.

The propositional attitudes are of the general form $X$ verb that $Y$, where verb represents a verb such as 'believe', 'hope', 'desire', and so on. However, it is also possible to express such attitudes non-literally. For example, the military analyst and novel writer, Tom Clancy, employs irony when he refers to the death of United States Marines in a 1983 suicide bomb attack in Lebanon. Here he portrays a marine communicating his belief of danger to his commanding officer:

"We safe, Sergeant?" "Sure, Lieutenant, I haven't felt this safe since Beirut." (Clancy, 1993, p. 261).

Another example is evident in Francis Anderson's address to the Public School Teachers' Association in 1901. Here, he outlines some proposed reforms to the New South Wales public school system, in which

\begin{abstract}
the inspectors would take their true place as men of "light and learning," the responsible and trusted guides and advisers of the teachers, the controlling brain of the whole organic system of education throughout the State (Anderson, 1975, p. 296).
\end{abstract}

In this extract, Anderson employs symbolism and analogy in his references to 'light and learning,' and 'the controlling brain'. Here, he expresses his belief that the new system would enable inspectors to move beyond their contemporary machinelike roles.

According to Heracleous (2006, p. 1), organisational theorists can use symbol and metaphor to mediate organisational understandings. He proposes a view of discourse as situated symbolic action for the interpretation of organisational talk and action (Heracleous, 2006, p. 160). In this view, the linguistic labels used in organisational discourse take on symbolic connotations and influence individual's perceptions of social reality. An example presented by Heracleous (2006, pp. 152153) describes a contest between the old-timers and newcomers of an organisation 
over the use of the term 'principal-led model'. This term symbolised different things to the two opposing groups. Symbolic connotations of this sort derive their meanings from the contextual details of the situations they describe. Their meanings go beyond literal interpretation; the sense attributed to such terms by some organisational members may not accepted by others.

\subsection{Conclusion}

Leadership is part of a complex reality that can be explained with or without recourse to leadership schemas. When leadership qualities and events are recalled, a narrative may or may not fit the phenomena being described. Faulty memories can arise from the reconfiguring of phenomena to fit existing schemas; some phenomena may be accommodated because they fit a schema and others ignored because they do not.

Narrative is made comprehensible by the use of socially mediated cognitive schemas in narrative structure. Importantly, the same narrative structure is employed in both factual and fictional accounts of events in situational narrative. One significant difference between situational narrative and grand narrative lies in the use of propositional attitude sentences in situational narrative. In contrast, the generalities of grand narrative rely on theoretical propositions in order to take on their overtly theoretical function.

The folk-psychological world of propositional attitudes does not translate well to the theoretical world because propositional attitudes suffer from the ambiguity of their intensional terms and the risk of fallacy. Yet it is very difficult if not impossible to give first-person reports that explain day-to-day organisational action, without also referring to propositional attitudes. Whilst the physical world expresses itself narratively through symbols, it expresses much more than can be comprehended. The legitimate question that may be asked is why this story about human interaction in the physical world and not another? 


\title{
Chapter 6: Leadership as information processing
}

\begin{abstract}
Whilst leadership sits comfortably within the special cases described by situational narrative, it is not amenable to global generalisations in the guise of formal, rule-governed explanations. Leadership may be described narratively, in an informal and context-dependent manner. However, attempts to develop formal, context-free theories of leadership have been marked by problems of definition and a lack of predictive power. Here, it is argued that the nexus of scientific and narrative accounts lies in information space, which may be described in both naturalistic and phenomenological terms. That is, leadership may be described according to the ambiguous information content of propositional attitudes; and organisational functioning according to the logically incomplete, causal-computational information content of the physical world.
\end{abstract}

\subsection{Introduction}

In the previous chapter, I argued that the specifics of situational narrative do not readily translate to the theoretical generalities of grand narrative. This is the case, also, for narratively conveyed accounts of leadership. In this chapter, I argue that formal leadership theories will always be inadequate to the task of explaining leadership.

Leadership is a socially mediated concept, one that is assumed to have causal significance for individuals and organisations. The leadership concept hinges on what it means to say that one person or another 'displayed leadership', or that it was so-and-so's 'leadership that improved that school', or that a school is foundering 'because of a lack of leadership'. Reports such as these express the theoretical perspectives of people - people who may include organisation members such as teachers and support personnel, formal positional leaders such as school principals, clients such as students and teachers, and people external to a school but with an interest in its performance, such as the media, researchers, or directors of educational systems. 
The implication of such statements is that leadership can be described by a set of principles or rules. Just as the material world can be described by a set of rules (e.g. the laws of physics), leadership is presumed to follow a set of causal rules of the type, "If $\mathrm{x}$ then y". If 'good' leadership, then 'better performing school'; or if 'lack of leadership' then 'poorly performing school'. The difference between the laws of physics and causal statements of that sort lies in the amenability to measurement and prediction of the former and the ambiguity of the latter. Whilst we may not possess a comprehensive theory of causation, nor know exactly what mass is in conceptual terms, we do possess scientific theories and research methodologies that can assess the outcomes of counterfactual cases. That is, the laws of physics enable the consideration of counterfactual antecedents, which specify events contrary to that observed and from which alternative world consequences can be inferred (Pearl, 1996). For example, by referring to the laws of physics, the payload of a space shuttle, as specified in customer requirements, can be assessed according to the trajectory required for rendezvous with an orbiting object such as the International Space Station.

The contrast between counterfactual cases of the sort mentioned in the previous paragraph and counterfactual leadership scenarios could not be more stark. To make a statement of the sort: "Person or organisational hierarchy " $\mathrm{X}$ " produced outcome "Y", implies that it is known that the counterfactual antecedent, having person or organisational hierarchy "A" would have resulted in, say, outcome "B". Claims of this sort may be found in formal theories of leadership.

\subsubsection{The lack of predictive power in formal leadership theories}

Formal leadership theories lack the general applicability and predictive power common to theories of physical science. The study of leadership has been plagued by the epistemological problems of logical positivism and operationalism; the mathematical reality of modest statistical associations; inbuilt cultural biases; confusion over feudal, bureaucratic and democratic organisational norms and structures; a pragmatic focus on weak social forces; and a substantial unexplained variance in leadership outcomes (see Bass, 1985, pp. 6-7; Foster, 1986, pp. 3-8; 
Watkins, 1986, pp. 3-32; Rizvi, 1986; Fiedler and Garcia, 1987, pp. 13-22; and Lakomski, 2005, pp. 5-10).

According to Fiedler and Garcia (1987, pp. 20-21), a lack of predictive power is evident in trait theories of leadership, theories based on leader behaviour, and structural theories that explain how situations can inhibit or support productive decision-making by leaders. This deficiency in predictive power is due, they say, to the exclusion of personality and individual differences from those theories. In contrast, they claim that the predictive power of the contingency model of leadership has been demonstrated repeatedly in research findings. However, upon closer examination, the two sources they cite in support of this claim carefully restrict the applicability of findings that might appear to support contingency theory.

From their meta-analysis, Strube and Garcia (1981) conclude that the predictive power of the contingency model receives strong statistical support. However, they also present a number of important disclaimers to their conclusion; namely, the restricted nature of the research, being focused mainly on military, business and college groups; mid-life age groupings; and group performance rather than individual performance. Peters, Hartke and Pohlmann (1985) question the veracity of the claims made by Strube and Garcia (1981). They open their argument by acknowledging the controversy surrounding Fiedler's contingency theory since its inception. Their metaanalysis includes findings that both support and contradict contingency theory. Importantly, they point out that the majority of empirical support for the theory comes from laboratory studies; and the results fall short of providing full support for the theory that fails to account for the dynamic interactions between leaders and the environments in which they function. Contingency theory is at the very most an incomplete theory.

Fiedler's contingency leadership theory, though described by some as 'useful' is found by several studies to be lacking in predictive power (Hill, 1969; McMahon, 1972; Moberg and Koch, 1975; Utecht and Heier, 1976; Wooton, 1977; Vecchio, 1979; and Meilich, 2006). In particular, Moberg and Koch (1975) caution against the theoretical and practical value of aggregated contingency models, applied in a 
universal way to the generalisation and oversimplification of complex organisational practice. Korman and Tanofsky (1975) contend that neither contingency nor noncontingency models can explain leadership in all its complexity. They suggest that whilst a formal theory can serve as an aid to problem identification and conceptualisation, any formal theory of leadership will always be incomplete. In Hill's (1976) analysis of the predictive power of contingency leadership theory, it was found that statistically significant correlations were not evident in the interacting and co-acting groups of two large organisations, an electronics firm and a hospital. Vecchio (1979) also found in a study of discriminating dyadic relations between leaders and subordinates amongst Air Force personnel, that contingency theory had doubtful predictive power and validity.

In a retrospective study of "certified" successful military leaders, Utecht and Hieier (1976) found that such leaders did not fit neatly within the parameters of contingency theory. Concluding that the contingency model was no better than pure chance in predicting success in military leadership, the authors acknowledged that their conclusions were at odds with the reported predictive success of contingency theory in other settings. Thus, they understate the significance of their findings, given that Fiedler's original studies were conducted with military groups. Utecht and Hieier suggest that the differences in the reported utility of contingency theories may be the product of biases due to variations in subjective phenomenal perception. This may be understood as an acknowledgement of the opaque nature of individual consciousness, as well as the influence of cultural norms and pre-existing theoretical perspectives on the narrative accounts of historians and interview subjects.

Wooton (1977) concludes that both phenomenological and deterministic approaches to management are needed in the study of leadership. He accuses contingency models of management of taking too much of a deterministic stance, emphasising a purposeless adaptive role for managers rather than a purposeful, creative role. To say that 'good leadership' makes a difference to an organisation's performance rests on phenomenal explanation, which differs in significant ways from naturalistic causal explanations. 


\subsubsection{Cause, effect and explanation}

In the children's picture book, Who Sank the Boat? (Allen, 1996), a donkey, a cow, a sheep, a pig, and a mouse, decide to go for a row in the bay 'for no particular reason'. As each animal climbs into the boat, the causal question is put to the reader, 'Who sank the boat?' The inherent difficulties of causal explanations become apparent in this simple story, in that several different causal explanations may follow from the one scenario, depending on where the focus of attention is placed by the reader.

Hume's doctrine of cause and effect has strongly influenced the modern naturalistic conception of cause. As Morris (2001) explains, Hume offers two definitions of cause:

- 'An object, followed by another, and where all objects similar to the first are followed by objects similar to the second', and

- 'An object followed by another and whose appearance always conveys the thought to that other' (Hume, 1975, pp. 76, 77).

Hume's cause comes before effect and the two are joined temporally by necessary connection, albeit one perceived inductively from experience. The first definition describes that which is evident in external impressions; that is, as a perceived connection. The second definition describes that which is evident through internal impressions; that is, as a felt connection.

Aristotle's doctrine of the four causes differs from Hume's by attributing cause to substances rather than to events. Aristotle's main interest seems to be in causes or explanations as answers to the question, 'Why?", whereas Hume's concern is with fixing the precise meaning of the terms cause and effect.

According to Hocutt (1974), the English word 'cause' means 'productive agent' or 'productive event' and can be equated with Aristotle's efficient cause. He argues that the Greek word aition is ambiguous; it has different senses, which cannot be captured by the word 'cause'. This is a point that seems to have the agreement of 
Cohen (2003, 2006). However, whilst Hocutt suggests that the English word 'because' could preserve the various senses of aition applied by Aristotle, Cohen suggests that it would be better to disambiguate the term through the use of four different English phrases such as in the following examples (Cohen, 2006, in quotations, bold type in original):

\section{Efficient aition: 'A carpenter is what produces a table'. \\ Material aition: 'Wood is what the table is made out of'. \\ Formal aition: 'Having four legs and a flat top is what it is to be a table'. \\ Final aition: 'Eating on and writing on is what a table is for'.}

Here, it is evident that the temporal relation of cause and effect, as emphasised by Hume, is not present in all of the four cases shown above. The only aition that could require cause to precede effect is the efficient aition; the other three cases, whilst continuing to imply a connection of two objects, are explanations that do not require antecedents and consequences in a temporal sense. Some philosophers such as, for example Aristotle and Bertrand Russell, have found that temporal relations pose the inconvenient question of short or long time-intervals between cause and effect; and the problem of joining a single past event with a single present event (Wilkie, 1950). The word 'cause' may be spoken of in terms of both antecedent and contemporaneous causation. If we apply the word 'cause' to an antecedent, we are faced with the problem of the temporal asymmetry of the universe due to the laws of thermodynamics (Davies, 1995, pp. 222-223). However, fixing the cause of a particular effect requires a contemporaneous definition of the effect (a conclusion reached by Aristotle). In the case of efficient aition, since a definition is also a type of explanation, it follows that explaining an object is synonymous with its contemporaneous cause.

Viewing Aristotle's four causes, not as 'causes', but as 'explanations' enables his logical theory, as presented in the Posterior Analytics (Aristotle, 1994c), to be joined to two other Aristotelian works: the Physics (Aristotle, 1994b) and Metaphysics (Aristotle, 1994a); thus achieving a coherence of the three works that is otherwise absent. Hence, the theory traditionally labelled 'Aristotle's theory of causes' would 
be better described as 'Aristotle's theory of explanations' (Hocutt, 1974, p. 385). Aristotle's notion of cause depends on the notion of explanation, and there is substantial evidence for Aristotle taking the view that all satisfactory scientific explanations are formal explanations (Hocutt, 1974, p. 390). As such, they carry a heavy theoretical burden in that describing an event is tantamount to explaining the event. However, a formal explanation at one level of analysis may become a material explanation at a lower level. For example, to explain eye colour formally in terms of genetic dispositions may be equivalent to explaining eye colour in terms of the material properties of deoxyribonucleic acid.

Material 'cause' is also related to efficient 'cause'. Aristotle's theory of causes is commonly illustrated according to a sculptor at work on a statue:

\begin{abstract}
We take a sculptor at work on a statue; the marble block is the material cause, the action of sculpting the efficient cause, the formal cause is the shape of the statue, and the final cause is the purpose for which the statue is intended. The only problem with this illustration is that it does not appear in Aristotelian text (Todd, 1976).
\end{abstract}

Aristotle uses a variety of illustrations to convey the distinction between the four senses of aition. He uses the statue illustration to demonstrate the relation between efficient and material aition. In modern physics, we find similar relations. For example, a rocket engine that has been engineered in a particular way (formal aition), mixes two chemicals (efficient aition), one being an oxidant and the other a reductant (material aition) to apply a force to a rocket so that the rocket overcomes gravity and accelerates upward (final aition). Any one or all of these senses of cause may be put forward as explanations for the flight of the rocket.

In Aristotle's Physics, 'cause' arises as an answer to the question, 'Why?' In the Metaphysics, Aristotle introduces the four senses of aition that may provide answers to such a question. However, Aristotle's most complete and definitive text on cause is found in the Posterior Analytics. In this text, he defines cause as the middle term of the syllogism. Since science aims at 'universal predications', the term 'effect' applies to the predicate term of a conclusion, but only when applied mechanistically 
with an efficient aition as the middle term of a syllogism. Hence, following Hempel and Oppenheim ${ }^{60}$, Hocutt (1974) substitutes the terms 'explanans' and 'explananda' for 'cause' and 'effect' respectively:

In summary, an explanation is a syllogism, a demonstration in which as explanandum, a statement of inherence of an attribute in a subject, is the conclusion, and in which the premises are the explanans (Hocutt, 1974, p. 391).

Hence, a naturalistic explanation may employ explanations that are efficient, material or formal; or a combination of these.

According to Hocutt (1974), Aristotle sometimes identified formal explanation with final explanation. However, attempts to construct syllogisms for final 'causes' show that they do not share the logical structure of efficient, material or formal 'causes'; they do not possess an unambiguous logical explanatory structure at all. Final explanations employ desires, purposes and beliefs, expressed in propositional attitude sentences. For example, I may take the action of mailing a letter to fulfil my desire to mail the letter; or with the purpose of the addressee receiving the letter; or because I believe the letter will arrive at its destination if it is mailed. In Chapter 5, I argued that such sentences have an inherently ambiguous structure, which when subjected to logical analysis, yield ambiguous results. Final explanations fall outside the province of natural science because they employ propositional attitude statements. If, as Aristotle sometimes says, final aition is identical with, or a part of formal aition, then it might seem that the problem may be solved by eliminating final explanations as a special type. This is the position taken by eliminative materialism. However, substituting an unambiguous statement in place of an ambiguous one is ultimately an arbitrary act.

It seems that efficient, formal and material explanations can be satisfactorily lumped together. However, final explanations have unique properties that render their elimination or assimilation into the other three arbitrary. Zimmerman (2001)

\footnotetext{
${ }^{60}$ Here Hocutt (1974) is careful to distance himself from the logical positivist philosophy of Hempel and Oppenheim.
} 
argues that emotionally animated propositional attitudes (which he labels alternatively as 'reactive attitudes') play an important part in 'rationally optional' shifts of attention. Whilst the origins of some emotional responses may be inaccessible to rational criticism, others may be consciously accessible, at least in part. Hence, emotional dispositions may be placed on a continuum according to their accessibility to rational evaluation (Zimmerman, 2001, Rorty, 1980).

In figure 6.1 below, the emotionally animated propositional attitudes are placed on such a continuum, with those that are explicitly known shown at (1) through to those that are tacitly known at (5):

\section{Figure 6.1 Continuum of explicit and implicit propositional attitudes}

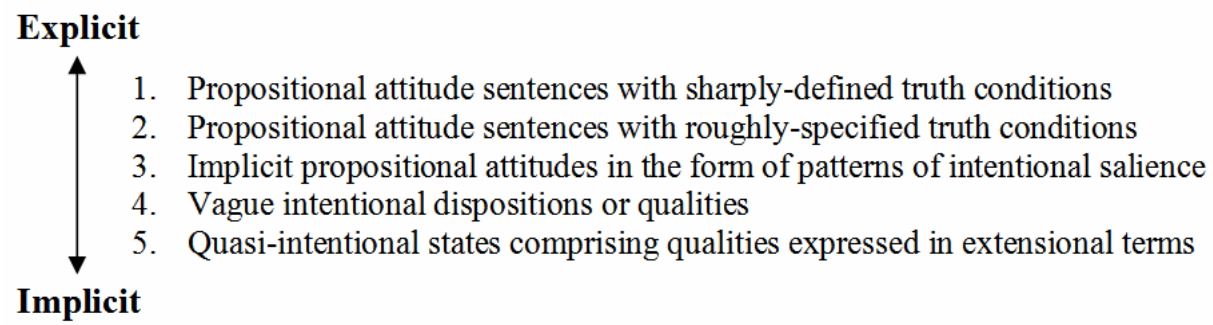

Cases 1 and 2 above involve emotions in explicit ways. In cases 3, 4 and 5, the reactive emotions (such as, for example, resentment or anger) have an implicit cognitive role by shifting attention from one set of facts to another and heightening awareness to new patterns of salience. However, to qualify as a rationally required shift in attention, the emotional component must be optional so that an emotional response may be accepted, or rejected as being inappropriate. Hence, it may be said that,

Emotions are valuable qua modes of attention for creatures like us, who live in highly variable worlds and whose behaviour towards each other is not rigidly stereotyped, because logical considerations alone, even when supplemented by any of the well-recognised epistemic and methodological principles, 'do not determine salience, what to attend to, what to inquire about' (quoted from de Sousa, 1980, pp. 134-142) in contexts in which this kind of focal efficiency is crucial to the advancement and protection of basic interests (Zimmerman, 2001, pp. 534-535) 
It was argued in Chapter 5 that conscious experience instantiates phenomenal qualities. It also appears that implicit propositional attitudes, from patterns of intentional salience through to quasi-intentional states, exhibit a strictly phenomenal character.

In summary, whilst modern science readily accommodates causal explanations in terms of efficient, formal and material explanations, it does not similarly accommodate final explanations. To figure final explanations in the form of propositional attitudes, emotions, or phenomenal experience in causal terms is fraught with unresolvable difficulties. The rule-governed, universal generalities of science cannot accommodate the rule-free, contextual details of phenomenal complexes.

\subsubsection{Phenomenal experience in narrative}

The distinction between rule governed events and events that transcend rules is one that appears within the narrative of the motion picture Matrix Trilogy. Here, the narrative posits a rule based world, created by machines and governed by computer code, but transcended by a greater world of freedom, hope, belief, and love. The central character, Neo, learns to enter the computational world of the Matrix whilst simultaneously circumventing its rules. Paradoxically, he is able to view the computational code of the Matrix within his mind because believes he cannot. Hence, the story portrays the ambiguity of the propositional attitudes.

Accounts of life in schools may be described in dispassionate causal terms by researchers, system level administrators, and policy makers. However, those talking about their work in schools may speak in terms of intentions such as purpose, desire and hope. When school principals or teachers give narrative accounts of events in their schools, they report their beliefs and desires by means of propositional attitude sentences. Consider this account, by the Principal of Eucalypt Primary School, concerning the question of shared vision in her school:

The arts community are involved in environmental issues and they have asked me how can they be involved, how can they influence the direction 
of the next school charter. Because, they have their agenda of instrumental music, choir, full performance, recycling, they want policies made about the whole gamut of environmental issues, they want to know how they can influence and be part of the school charter, working on what they want to do. They want to feed information in but don't really want to do the work, and I think that is probably going to be the direction of a lot of schools now. They need to get the information and outsource. They need to learn how to get the correct information from parents and that's one of the things that I said to them at the meeting the other night: 'Your opinions are valued but they may not necessarily reflect the opinions of parents in the wider school community' (Interview B5).

In this account, the principal compares her beliefs and theoretical perspectives with those of the 'arts community', which is a subset of the wider school community of parents and children. As Bach (2000) has said, the logical properties of the beliefs reported by people are not fully determined. Rather, they are delimited by the semantic contents (meanings) of the 'that' -clauses they contain. In the above example, the statement, 'that is probably going to be the direction of a lot of schools now', appears to delimit the question of vision to a question of parents' wants and the resources available to meet them. However, the logical content of this statement lies not in the statement itself, but in the details provided by the other sentences in the principal's statement, and many unstated details. Simply put, there is more to belief than that expressed in theoretical propositions.

The propositional attitudes of values, wants and agendas give added meaning to the theories held by people as do references to subsidiary theoretical propositions. When applied to the detail of school situation, the generalities of theoretical propositions, alone, are unable to explain that particular situation. This requires that the complete array of information, inherent in the situation, be formally compressed into a set of rule governed sentences. However, practical limitations ensure that only a portion of that information may be compressed in this way. Furthermore, there is no guarantee that those sentences will be governed by the fine-grained detail of the situation rather than the properties of the symbols themselves. 


\subsubsection{The computational character of sentences}

Symbolic sentence processing is formally construed as a linear process by which each symbol is processed sequentially according to logical or mathematical operations. Theoretical propositions and algorithms are processed in this way. According to Chaitin $(1969,1995,1999,2006)$ algorithmic information is that which can be described simply (or compressed). In contrast, non-algorithmic information is so complex that the information relating to a given event or situation cannot be described by anything less than the situation itself. There is no systematic or reasonable way to reproduce non-algorithmic information as it appears to be random and devoid of pattern.

Bennett (1988) takes this view further by classifying highly complex situations as, both, 'algorithmically random' and 'logically deep'; whereas, logically shallow situations are those that can be produced quickly by a systematic procedure or algorithm. Logically deep situations are those that require many successive steps of information processing. Much more work must be done to produce or explain a logically deep situation when compared to a logically shallow one:

Deep objects cannot be quickly produced from shallow ones by any deterministic process, nor with much probability by a probabilistic process, but can be produced slowly (Bennett, 1988, p. 227).

Bennett's theory of logical depth and physical complexity places limitations on deterministic physical theories. To explain a logically deep state of affairs firstly requires recursive feedback processes that are repeated many times; and secondly, requires the evaluation of the process itself. The former proceeds towards more precise descriptions whilst the latter proceeds towards descriptions that fit the situation better.

Whilst deterministic algorithms are helpful in reducing some aspects of nature to speedy computations, other aspects take more time and must be described holistically. This is in keeping with supervenience relations and emergence in the (material) universe. That is, higher level events supervene naturally on lower levels so that 
physics per se cannot causally determine the outcome of human creativity; rather it creates the 'possibility space' to allow human intelligence to function autonomously (Ellis, 2005).

Whilst physical theories are essential to our understanding of human cognition, they cannot fully explain our cognitive capacities, consciousness, or our autonomous freewill choices. The decisions made by groups, for example, do not necessarily line up with the individual decisions of their members. Supervenience relations appear to apply to group decision-making processes so that

knowing what the group members individually think about some proposition does not generally tell us how the group collectively adjudicates that proposition (List, C. and Pettit, P., 2006).

However, List and Pettit caution that this does not amount to a form of group agency.

Leadership is often described as the speedy reform or improvement of an organisation as determined by the conscious deliberations of the leader. Described in this way, leadership becomes the outworking of conscious thought, rather than nonconscious cognitive processes. Penrose (1989) proposes that our non-conscious processes operate algorithmically according to patterns established by genetics or experience. If this were the case, it would follow that the information underlying those processes is compressible, and that those computations determine particular outcomes. However, in seeking an explanation for conscious cognitive processes such as inspiration and creativity, Penrose also suggests that conscious thinking may depend, at least in part, upon non-algorithmic processes, which are uncompressible and subject to Gödel's incompleteness theorem. If this is the case, logically deep, algorithmic, non-conscious cognitive processes may be partly due to genetic dispositions obtained over a long evolutionary period, but also due to the lifetime learning of an individual.

These conjectures of Penrose (1989) are vigorously challenged by Grush and P. S. Churchland (1995). Much of their criticism hinges on a rejection of Platonism, deficiencies in the epistemology of mathematics, and the tendency for human thinking to contain errors of reasoning. Nevertheless, they admit that 'consciousness 
is a problem' and that many fundamental questions about human cognition have not yet been satisfactorily answered. Whilst they criticise the speculative nature of Penrose's argument concerning a non-algorithmic aspect to human cognition, they admit that they cannot demonstrate that a connection between quantum uncertainty and consciousness is false. Yet, Penrose's central claim is that a connection of this sort, whilst neither confirmed nor refuted, should be given serious consideration. Instead, Grush and Churchland (1995) settle on the argument that their eliminative materialist position has more epistemological and empirical support than Penrose's.

Central to Grush and Churchland's (1995) commitment to eliminative materialism is the dismissal of any limits to human reasoning and empirical discovery. Their argument depends in part on 'errors in workaday human cognition' and weaknesses in an argument for the 'soundness' of mathematical reasoning, which together throw doubt on the reach of Gödel's theorem as interpreted by Penrose. Most importantly, however, they claim that there is a lack of empirical evidence for non-algorithmic processes in the universe, and that Penrose's argument is 'far fetched'.

However, Chaitin $(1988,2005,2006)$ has shown that non-algorithmic features are, in principle, undiscoverable, such as in the case of the uncomputable number, 'Omega' and his 'Diophantine Equation'. Omega is an infinitely complex number that gives the probability that a computer program will halt. No finite computer can ever calculate Omega, yet it can be rigorously defined, mathematically. Chaitin's Diophantine Equation is a huge equation containing seventeen thousand variables, which may yield either a finite or an infinite number of solutions. What the Diophantine Equation demonstrates, is that there can be no systematic procedure for deciding the answer to well defined mathematical questions in advance. According to Davies (1992, pp. 130-131), mathematical complexity such as that found in Omega and the Diophantine Equation, can be convincingly extended to physical systems, when those systems are interpreted as computational systems.

Chalmers (1995) agrees that Penrose does not succeed in explaining consciousness, particularly concerning Penrose's argument for the role of 
microtubules and quantum gravity. However, he thinks Penrose has succeeded in showing that the powers of human reasoning cannot be captured by any formal system. The ability to determine the soundness or consistency of (computational or cognitive) system is not a requirement for a successful Gödelian argument. Chalmer's interpretation of Penrose's book, Shadows of the Mind, therefore, undermines a significant portion of the argument posed by Grush and Churchland (1995). If we were purely computational systems, the act of assuming that we know we are sound or consistent, in itself, gives rise to a Gödelian contradiction.

Explaining consciousness, according to Chalmers (1995) requires that the following questions be answered:

(1) What does it take to simulate our physical action?

(2) What does it take to evoke conscious awareness?

(3) What does it take to explain conscious awareness?

(Chalmers, 1995)

There are three possible positions that may be taken regarding each these three questions:

(C) Computation alone is enough

(P) Physics is enough, but physical features beyond computation are required

(N) Not even physics is enough.

(Chalmers, 1995)

A large variety of positions arise from different combinations of (1), (2), (3) with $(\mathrm{C}),(\mathrm{P})$ and $(\mathrm{N})$ and Chalmers goes to the trouble of specifying the general positions of various writers in this regard. Chalmers' own position is that computation and physics alone $(\mathrm{N})$ are not enough to explain consciousness, but computation $(\mathrm{C})$ is sufficient for explaining (1) and (2). 
Whilst a connection between reason and causality remains, it continues to be true that 'compression algorithms must ultimately account for all the non-random data' (Evers and Lakomski, 2001, p. 506). Nevertheless, as Chalmers (1996) points out,

there are two classes of facts that do not supervene logically on particular physical facts: facts about consciousness and facts about causation (Chalmers, 1996, p. 152).

As mentioned earlier in this chapter, the metaphysics of causation continues to pose difficult problems, and the possibility remains that the nature of experience may have some role in its explanation. It may turn out that the (current) mysteries of consciousness and causation are related in some way. Causality is described in terms of the relations between physical entities - relations that become increasingly complex as the number of entities increases ${ }^{61}$. However, it is not at all clear what causation relates; what it is that is doing the causing. What Chalmers proposes towards a solution to the question of consciousness is that it be explained according to causal and phenomenal properties, and that the link between the two lies in information space. In this view, a given information space may be realised both physically and phenomenally ${ }^{62}$.

The phenomenal accounts given by school principals and teachers also rely on references to beliefs, desires and intentions. Human behaviour can be correctly explained, and sometimes correctly predicted by reference to propositional attitudes, despite their ambiguity (Israel, 1987). In such cases, it seems that a commonsense (folk-psychological) theory of rational action is employed to explain and predict events. As BonJour (1998, p. 162) has said, it is obvious that we humans can consciously represent and think about a wide variety of entities, properties, and relations that are external to our cognitive processes. However, while we clearly possess this capacity, explaining how we are able to do this is much more perplexing.

\footnotetext{
${ }^{61}$ Ultimately, when the relations between all the physical entities in the universe are considered in this way, what we end up with is a giant causal flux (of the sort proposed by the pre-Socratic philosopher, Heraclites).

${ }^{62}$ This view is a version of property dualism, which Chalmers (1996, p.128) prefers to label as 'naturalistic dualism' because of its continuing commitment to natural explanation and physical causation.
} 
Only voluntary cognition can be rational, and what makes it rational is that we are able to 'monitor it introspectively, reason about it and change its course' (Pollock, 2006). This requires knowledge.

If leadership is rational action, it must utilise knowledge - knowledge of people, or knowledge of an organisation and its context. A film director needs to know the capabilities of his or her actors; in time of war, a military commander needs to know his enemy; and the director of a trading company needs an appropriate knowledge of the market. Similarly, a school principal is expected to possess an appropriate knowledge of the management and operation of a school and the social knowledge needed to facilitate the shared ideals and purposes of a school community (Sergiovanni, 1994).

This kind of knowledge is only leadership knowledge to the extent that it can be identified with actions typically taken by those in formal leadership positions. In this sense, leadership is nothing more than a particular apportioning of the work to be performed in the organisation. When leadership is conceptualised in this way, judging the extent to which leadership has been performed is simply a matter of judging the extent to which the expected performance has been achieved. Indeed, this is the approach that has been taken by the Victorian Department of Education with its Developmental Learning Framework for School Leaders (2007, pp. 2, 11-12), which is designed to be used for the purposes of guiding performance and development reviews, principal selection, the coaching and mentoring of principals, leadership induction and succession planning. The framework describes the leadership capacity of teachers and principals in terms of capabilities, knowledge, skills and dispositions.

Of more interest to a study of distributed leadership, however, is the distinction between the cognitive acts of leadership and other acts of human cognition. Some believe that leadership is a concept that resides exclusively in the minds of followers (Eden and Leviatan, 1975; Lord and Emrich, 2000), while some see it as a relation between leaders and followers (Popper, 2004). Others believe that principals can resolve the tension between teacher evaluation and other demands by viewing 
cognition as a socially distributed phenomenon (Halverston and Clifford, 2006); or that leaders construct their own personal view of leadership from cognitive predispositions and a process of situated cognition (Eddy, 2005). Joining human cognition to the concept of leadership assumes that a relationship exists between these two concepts.

Any human act is, by default, a cognitive act because it will involve varying degrees of conscious deliberation and reason, automatic and routine responses, and the activation of emotion and feeling. Hence, any leadership act must also be a cognitive act. It does not necessarily follow, however, that a cognitive act can be a leadership act - for that would depend upon how that act is perceived and classified as leadership. It seems that this is a question of phenomenal qualities, rather than physical causes; and, insofar as it can be expressed in propositional attitude sentences, the phenomenal sits more comfortably in folk psychology than in science.

\subsection{Cognition as information processing}

The proliferation of books about leadership and everyday use of the words 'leader' and 'leadership' are testimony to the emphasis placed on leadership in folk psychology. Use of the term leadership can be readily accepted without much in the way of critical evaluation; and the use of the terms 'leader' and 'leadership' is rarely questioned when applied to contexts such as military conflict and sporting contests. Phrases such as 'he is a true leader' and 'because of her leadership' are commonly used. Middenhorp (2004) echoes this propensity in the following example. Interestingly, he also asserts the often implied (but not necessarily stated) mystical qualities of leadership.

Infinitely more elusive than astrology, tremendously more arcane than religion, leadership in all its effable glory preoccupies us like no other subject. From John Howard to Mark Latham, from Nelson Mandella to Slobodan Milosevic, from Kerry Packer to Mick Malthouse, leaders good, bad and ugly are nothing less than the glue of human history (Middenhorp, 2004). 
At the time this extract was published, John Howard, Prime Minister of Australia, was opposed by Mark Latham as Leader of the Opposition; Nelson Mandella and Slobodan Milosevic represent moral opposites; and Kerry Packer, a wealthy businessman and media tycoon, is contrasted with Mick Malthouse, a legendary Australian Rules footballer and coach. Middenhorp's use of opposites here attributes a sort of universal, spiritual quality to leadership, albeit one that is stripped of moral significance. This is an example a folk psychological narrative that draws on the reader's contextual knowledge of differences to express phenomenal leadership qualities.

Leadership occupies a large part of the thoughts, actions and narrative accounts of people in modern western society. It features prominently as an explanation for the heralded qualities of many kinds of communities and organisations. Leadership is given causal significance as an explanation of past events and the construction of plausible futures so that outcomes of great significance are attributed to the actions of leaders.

\subsubsection{Schemas as aids to information processing}

The attributions of leadership, given in the narratives of folk psychology, are symbolic in character. The grand narratives of leadership express particular theoretical viewpoints constructed from an array of theoretical objects, ideas and actions and encoded as representations in memory. Hence, various symbols of leadership are employed together with other symbols and expressed through the semiotic systems of language:

Everything used by a multi-levelled subjectivity to turn graphemes into meaning is 'inscribed' in some text or other, some semiotic system. Even our apparently direct access to the world arrives through narrow sensory codes and channels, and vast quantities of neural pre-processing (Broderick, 1994, p. 126). 
Relationships hold between narrative texts and our neural architectures and those relationships are mediated by cognitive schemas, also known as 'mental models'. Schemas enable people to recognise and interpret leadership narratives; and enable the theoretical viewpoints of narrators and readers to interact.

Leadership narratives are interpreted according to the meanings evoked by the reader, and that meaning may well differ from the meaning held by the writer:

\begin{abstract}
A text is just an array of abandoned lexemes until it is activated by semiotic systems of textual codes, frames and encyclopaedias operating inside and around its readers' (Broderick, 1994, p. 126, italics in original).
\end{abstract}

When narrative texts are activated by the reader, those activations arise from the reader's cognitive dispositions, which may be understood as a set of initial conditions. Those dispositions arise from a combination of genetic inheritance and the prior cultural and environmental experiences of the individual. Leadership schemas acquire common currency as they emerge from the culturally mediated cognitive activities of people. When such socially derived leadership schemas are employed, they function together with schemas applying to various cultural conventions and understandings. Particular combinations of cognitive schemas will apply to particular types of situation.

The information constituting a schema also constitutes a disposition. This information is activated semantically by particular types of external features or contexts. Evolution by natural selection, and the structures that constitute human cognitive structures, are related information processing systems (Sweller, 2006). In human cognition, schemas provide a means for quickly classifying and processing information. Those schemas arise partly from the social transfer of information from individual to individual. As humans 'imitate other people, listen to what they say and read what they write' (Sweller, 2006, p. 438), information is interpreted, and therefore transferred in part, from the long-term memory of one person to the longterm memory of another. 
Mirror neurons play an important part in this process of information transfer, or imitation. Mirror neuron systems function as observation-execution systems, which are activated whenever an individual observes or executes an action. Merely listening to sentences that describe actions is enough to activate mirror neurons, according to some empirical evidence (Tettamanti et al., 2005). Mirror neuron activation in experimental subjects is heightened whenever subjects observe actions and imitate them in action. It seems that the process of imitating the actions of others, either by direct observation or indirectly through narrative, is an important to our ability to borrow and reorganise information and store it in cognitive schemas.

Schemas enable us to classify multiple elements of information according to previous patterns of use and enable us to accommodate new information as they are reorganised. A schema may assist memory, when fitting items are encountered, or distort memory, if it must be reorganised to accommodate new information. In cases of this sort, memories are distorted to become more consistent with an existing schema, rather than the other way around. Hence, we do not keep complete and exact copies of the situations we encounter in memory. Rather, we remember a version of that situation that is consistent with our classifications of prior experiences. The original situation is not forgotten per se, but is replaced with something else more fitting to the schema (Hill, 1981, pp. 207-8). Schemas can help memory by allowing the reconstruction of partly forgotten information; or distort or recast memory when a virtual situation (or potentiality), consistent with an existing schema, is inferred from an actual situation.

It seems that phenomenological experience interacts with the physical processes of cognition to fashion our perceptions and memories of life experiences. The multilevel personal experiences of individuals are mediated with the views we simultaneously hold about other people, or particular situations (Gallese, 2005). In this view, both physical information and phenomenological information arise from the neural processes of human cognition, with the phenomenological information being composed of a uniquely subjective interpretation of reality - as viewed from within conscious experience. 


\subsubsection{The physical and phenomenological realisation of Information}

Human cognition involves the processing of information, which has both syntactic and semantic structure. Its syntactic structure is realised grammatically according to rule governed relations between symbols; its semantic structure arises from its correlation with a system of physical or conceptual entities (Shannon, 1948). Since the syntactic structure of information arises from its level of organisation, it is vulnerable to randomisation due to 'noise,' disruptions to transmission and so on. Hence, syntactic structure is equivalent to the physical concept of entropy (disorder) and is therefore analysable, in principle, by stochastic (probabilistic) processes.

Syntactic information can be understood in terms of abstract spaces. These spaces comprise a certain number of possibilities from which a specific information state is selected. Though not part of the concrete physical world, these spaces can be realised in the world. For example, a light switch constitutes a two-state information space, with the two physical states, 'up' and 'down,' corresponding to the two information states, 'off' and 'on.' However, the two physical states correspond to the two information states only to the extent that the information can be processed. Hence, in the case of the electrical connection between the switch and the light bulb being damaged, the causal pathway is broken, the information cannot be processed, and the bulb is not lit even though the switch is 'down.'

Drawing from this syntactic view of information, Chalmers (1996, p. 283-284), argues that information can also be realised phenomenologically, whereby states of experience fall into information spaces. In this view, when we perceive natural patterns of similarity and difference between phenomenal states we also obtain an abstract information space comprising a two-dimensional relational structure. Whilst physical information is realised causally, phenomenal information is realised according to the intrinsic qualities of experiences and the similarity and difference relations they bear to each other.

Take the example of My Country, written by the Australian poet, Dorothea Mackellar. In this poem, Mackellar compares her knowledge of England and 
Australia in terms of intrinsic qualities, which she maintains cannot be understood by those who have not had the same sorts of experiences. Of one she says,

Strong love of grey-blue distance

Brown streams and soft dim skies

I know but cannot share it,

My love is otherwise (Mackellar, 2003).

Of the other, she says,

An opal-hearted country,

A wilful, lavish land-

All you who have not loved her,

You will not understand (Mackellar, 2003).

In this poem, Mackellar describes nature in a phenomenal sense, so that the words and rhythm of the lyric poem may evoke understanding in the reader - but only if the reader has had similar experiences; experiences that enable such qualities to be grasped. Nevertheless, the climate and landscape of England and Australia can also be described in a physical sense; that is, in terms of the causal effects of climate, flora and fauna, and geology. Whereas the phenomenal is described in terms of differences, the physical is described in terms of structure and function, and in terms of causal antecedents and consequences.

The upshot of Chalmers' (1996) conception of syntactic information is that reality takes on a dual aspect; that is, an information space is realised both physically and phenomenally. Another example, a simple colour experience, may be analysed in terms of the causal effects realised in the neural representations of the brain. However, Chalmers suggests that it can also be analysed in terms of the perceived qualities of the colour as experienced in a phenomenal information space. Hence, information space is being cast as the link between the physical and the phenomenal $^{63}$.

\footnotetext{
${ }^{63}$ Chalmers restricts his analysis to syntactic information, but does not rule out similar treatments with semantic information.
} 
Nida-Rümelin (2005) argues that there are no phenomenal properties that can also be physical properties. She claims that it is possible for a rational person, with arbitrary physical knowledge, to understand both physical and phenomenal concepts; phenomenal properties can be grasped via phenomenal concepts and physical properties can be grasped via physical concepts (by people with the relevant physical background knowledge). However, this also requires a principle of cognitive transparency that joins both the understanding of concepts to the grasping of properties. This principle relies on the grasping of relations between concepts:

On my principle, a priori knowledge is not a matter of linguistic knowledge but rather a matter of grasping relations of necessity that hold between properties independently of our linguistic conventions and of our conceptual capacities (Nida-Rümelin, 2005).

Phenomenal properties cannot be grasped physically because their character partly depends on the hidden nature of microphysical entities. Such microphysical entities present a veiled reality that exists independently of the human mind, yet appear superior to the human mind because they are intrinsically unpredictable (Schins, 1997, p. 182). According to Nida-Rümelin's analysis, it is not possible to grasp phenomenal properties by means of physical concepts. This is another argument in favour of property dualism; albeit one that differs from Chalmers' (1996) argument.

Stent (2005) also questions the utility of monism, given the resilience of dualism in common thought. Whilst many other beliefs have fallen into disuse since the rise of modern scientific theorising, versions of dualism have persisted from predynastic Egypt to the present time. In Stent's view, the 'latter-day obituary notices of dualism are premature; they merely reflect the failure of modern devotees of monism to fathom the moral foundations of the mind-body problem' (Stent, 2005, p. 149). Dualism need not stand in opposition to naturalism.

The quantum duality of an electron is testimony to the natural complementarity of a physical entity. After Bohr (1933), the complementary features of an electron have been found to be conceptually irreconcilable; however, empirical methods can 
never show them to be irreconcilable. This ability for electrons to exhibit a duality of properties has been interpreted in a number of ways. According to the interpretation influenced by Bohr, known as the Copenhagen interpretation, the mere act of observation causes a ghostly superposition of worlds to collapse into a single concrete reality. If so, then the act of making an observation forces nature to "make up its mind' and adopt one reality or another. Another equally astonishing interpretation, known as the quantum multiverse, is that the universe divides into alternative worlds every time an observation is made. Either way, the mental realm of conscious thought has an important part to play in cosmic evolution (Davies, 2006, p. 259).

Referring to Chalmers' (1996) opposition to Dennett's (1991) acceptance of reductive functionalism, and Dennett's rejection of phenomenological concepts, Ross (2005) questions the relevance of ontological debates about the merits of monism or dualism. Whilst the ontological claims of Chalmers and Dennett differ considerably, according to Ross (2005), their methodology is identical. That is, they both accept that the only way to study consciousness is to search for

\footnotetext{
"organisationally invariant features" in neural and information-processing architectures, which are linked by psychophysical laws with types of dispositions on the part of subjects to report that they are having particular sorts of qualitative experiences (Ross, 2005, pp. 166-167).
}

However, Johnson's (2005), view is that ontology does matter, and that it may matter in the sense that the nature of mind may only be partly scientific in character. He makes the pertinent point that

cognitive scientists have not been able to agree on a single, satisfactory answer to the question of what the mind is. Instead, their statements on that subject remain manifold, unspecific, incomplete, and sometimes contradictory (Johnson, 2005, p. 4).

Johnson refers to a chapter that was to be written by experienced Biologist, Robert Haynes and included in The Mind as a Scientific Object: Between Brain and Culture 
(Erneling and Johnson, eds., 2005). Unfortunately, the chapter was not written due to Haynes' sudden illness and death. Haynes' conference paper, on which the chapter was to be based, expressed his commitment to the relevance of qualia. According to Johnson (2005), this viewpoint was the product of Haynes' 'hard-headed' practical experience as a biologist, and is similar to the view Chalmers (1996) arrived at by metaphysical analysis and introspection.

\subsubsection{A functionalist account of the phenomenal and physical}

The difficulty of reducing living things to the properties of the physical alone is a problem that also concerned Aristotle. In De Anima, Aristotle could not see how the soul could be reduced to the set of properties of a merely material body. His solution was to propose a metaphysical system composed of Form and Matter, with both being substantial. By this view, the soul of the living is Form, and the body, Matter; but without the soul being an attribute of the body. Hence, Aristotle's view is both monistic, in the sense that a substantial soul is inseparably connected to a substantial body; and dualistic, in the sense of the soul being more than the mere attribute of the body. This is similar to the relationship between information and matter, in that information is realised physically in some way, but has an existence that transcends the physical arrangement of matter ${ }^{64}$. Aristotle's view of the soul differed from the purely spiritual substance envisaged by Plato:

We are left with a concept [the soul] that made only the rather short passage from being a purely spiritual substance, captured in a body that is alien to it, to being a spiritual substance that finds its necessary location in the realisation that it constitutes a certain matter (Lawson-Tancred, 1986, p. 53).

Aristotle's biological-functional thesis (Lawson-Tancred, 1986) is that a complete account of the features and activity of living organisms must go beyond

\footnotetext{
${ }^{64}$ The information in an academic paper, for example, may be realised physically in symbols inscribed in ink on a page, as pixels on a computer monitor, as packets of data in a fibre-optic cable, as the wireless transmission of radio-waves, in $\mathrm{pdf}, \mathrm{rtf}$ or $\mathrm{html}$ format, as morse code, in the memories of the writer, and so on. The information is not restricted to any one physical representation, but exists physically in one arrangement or another. However, the conversion of information from one physical arrangement to another also requires the existence of an appropriate translator.
} 
their material constituents to the functions they perform. That is, we may not explain all the features of the activity of living organisms entirely in terms of their material constituents, but must also refer to their functions. This view persists in the modern biological conception of the structure and function of living things.

Aristotle insists that a natural, structural parallelism exists between soul and body so that the one and same activity of a person may be explained in two ways: Firstly, in terms of the matter that composes the body; and secondly, in terms of the form that composes the soul. In a similar way, Chalmers (1996) suggests that mind may be explained in two ways: Firstly in terms of the physical properties of the body; and secondly in terms of the phenomenal world of subjective 'feels', or qualia.

The eliminative materialist view is that mind can only be explained in terms of the physical. This requires that physiological explanations of brain function be equivalent to explanations of mind. Hence, an extreme materialist view requires the denial of the existence of consciousness (Popper \& Eccles, 1977, p. 98). In an eliminative materialist account of perception, the material neural arrangements of the person must somehow be arranged to represent the same pattern as the object perceived. However:

\begin{abstract}
Aristotle is demanding that there must be in the percipient something to account for its coming to be arranged at least in some way in the same pattern as that which is perceived, and that this cannot merely be one of the things of which the object is composed and which happens also to be found in the percipient (Lawson-Tancred, 1986, p. 36).
\end{abstract}

Recourse to the latest and most sophisticated physiology of modern neuroscience, as a substitution for the ancient Democritean and Empedoclean physiologies, will always be insufficient to the task of explaining perception. To explain human motivation, both a phenomenal account of intention and a physical account of action are required. It is necessary to explain the intentional together with the physical; otherwise we risk losing sight of what it is we have set out to explain.

In some ways, we process the information in our environment by means of the computational processes of the human brain. However, the parallelism between 
computing machines, such as a handheld calculator, and us can only go so far. People also have hopes, fears and interests. We are much more than computational devices:

\begin{abstract}
We are not merely calculators; and insofar as we are calculators, we are miserably bad ones. Every ordinary adding machine is superior to most of us. Indeed, we would not construct multiplication tables and systems of arithmetic if our brains could calculate. We construct methods of calculating with pencil and paper, and electronic brains, simply because we have not enough brains ourselves (Popper, 1982, p. 107).
\end{abstract}

In making sense of the world, we scaffold our thinking, store information and communicate with others by interaction with culture and environment. Some aspects of our knowledge are inescapably private and inexpressible, even to the extent that we are not consciously aware of the electrical and biochemical workings of our brains. Other aspects are communicated to others narratively in the stories of our experiences - stories that can be real or imaginary, fixed or fluid, or personally determined or socially negotiated:

We know from our own experience in telling consequential stories about ourselves that there is an ineluctably "human" side to making sense (Bruner, 1990, p. 55).

Meaning is important because the cultural dimension of human cognition depends upon it.

\title{
6.2.4 The global nature of information
}

The interpretation of meaning relies on the structure and coherence of the contexts in which meaning is made and transmitted. A school obtains meaning by reference to the society it serves; a school principal obtains meaning by reference to the students, parents and teachers of a school community; and a leader obtains meaning by reference to those who are led. The semantic information of meaning refers to global features; meaning can only be obtained by reference to context. This global characteristic also implies that semantic information cannot be reduced to that which is local. This may be one reason for why it is so difficult to get clear answers about nature from empirical investigations (Chaitin, 1997, p. 20). 
Sufficiently interesting theories cannot be obtained by mechanical means; a generic algorithm alone is not able to give meaning to the description of a problem. A reference to external reality is needed, if a problem is to be fully determined. We cannot construct a formal description of the entire universe because that would require reference to something outside the universe:

\begin{abstract}
If no formal system of axioms can represent completely even a small portion of reality, one is led to conclude that there is no immanent cause of truth in the world other than the reality itself. Reality obviously cannot justify its intelligible content. Therefore, one is led to the situation in which reality is the only source of truth, but it does not contain the causes of truth. So, either intelligibility of reality has no cause at all, or its cause is outside the (sensible) universe (Cacace, 1997, p. 39).
\end{abstract}

There are severe limitations to what humans can know about the universe. For example, the Michelson-Morely experiments indicate that humans cannot communicate at speeds faster than the speed of light; however, there is also experimental support for faster-than-light phenomena in nature (Suarez, 1997, p. 170-171). As long as we accept these sorts of seemingly contradictory experimental findings at face value, we must accept that nature will always be capable of causal effects that cannot be understood or reproduced by humans. The existence of nonlocal, cause-producing, faster-than-light influences, suggests the existence of a principle that is independent of space and time and which cannot be accessed by humans for practical purposes (Pliska, 1997, p. 118). The limitations to what we can know about the universe extend to our ability to understand the human mind, culture, organisations and leadership.

\title{
6.2.5 Consciousness and free-will
}

The concept of leadership is premised upon the concepts of concerted human action, and of free-will. In our everyday lives, it is common for us to explain our actions in terms of choice, that is, our free-will prerogative to do one thing or another in a given situation. We might also explain our choices in terms of what we believe to be true or not, or the rightness or wrongness of alternative actions, or in terms of what we intend to achieve or avoid. 
Popper (1982) argues that an indeterministic view of the world is necessary for us to explain voluntary movement in humans and other animals. In keeping with his metaphysical program, he suggests that voluntary actions can be explained in terms of inherent propensities, superimposed upon other physical propensities that have a 'more chance-like or equi-probabilistic character. By giving weight to certain possibilities, these propensities impose a systematic bias upon those possibilities (Popper, 1982, p. 210). The superposition of inherent possibilities, he says, plays an important role in classical physics in the form of loaded dice, osmotic pressure, and resonance. Therefore, we do not need to take a very large step to adopt the intuitive idea that free-will arises from 'a hierarchy of systematic and increasingly purposeful biases' (Popper, 1982, p. 210) ${ }^{65}$.

The concept of free-will is closely related to that of consciousness. Penrose (1989, pp. 405-449) argues that all machines, whether of parallel or serial architecture, operate algorithmically. That is, they implement systematic procedures, which are constrained by the laws of physics and the rules of mathematics. However, the action of consciousness, he argues, requires a non-algorithmic ingredient. In this view, conscious action is that which utilises common sense, makes truth judgements, enacts understanding, and appraises phenomena artistically. Unconscious action, however, is that which is automatic, programmed, algorithmic, and follows preset rules in a way that is not directly accessible to the conscious. Penrose's distinctions are fuzzy and holistic; they enable intuition and insight to be classified as unconscious processes. Whilst the conscious mind cannot access these processes, it can access their products. Penrose (1989, pp. 418-419) cites the example of Henri Poincaré who had been consciously seeking a solution to the problem of what he called Fuchsian functions. The story tells of him reaching an impasse and putting his conscious deliberations aside, only to have the solution come to him in a flash later as he was boarding an omnibus.

\footnotetext{
${ }^{65}$ In my view, Poppers' metaphysical program (which differs from his earlier demarcations of science and metaphysics) sits comfortably within a naturalistic coherentist epistemology. This program entails gathering all the 'true' aspects of the world - including but not restricted to its scientific aspects - into a unified picture. In this way, a 'truer' picture can be obtained, one that is at the same time more coherent and more closely represents the way the world as it is.
} 
Unconscious processes might be algorithmic processes, as suggested by Penrose (1989). However, identifying such processes would be extremely difficult if not impossible because the information held by humans is distributed amongst the many modules of the cognitive system. Logical thinking certainly occurs in conscious thought; however, Penrose believes this occurs through the manipulation of entire thoughts. Sometimes these manipulations have an algorithmic character, such as in the formal syllogisms of Aristotle or in modern Boolean logic; sometimes they do not, such as in Gödel's incompleteness theorems. What the theorems of Gödel, Turing and Church have shown, is that people are capable of dealing with situations of incompleteness and mathematical undecideability in ways that algorithmic processes cannot. Hence, it seems that we must be able to use non-algorithmic processes in the process of forming judgements, making decisions, and to focus our attentions on some features or problems in our environment whilst ignoring other features or problems.

Whilst algorithms provide an extremely powerful means for dealing with certain type of problems, some other means is needed to choose the most suitable algorithm for solving the most important problem. To choose that which is most suitable, or most important, are decisions of value aimed at obtaining the best possible answer. Such decisions may be made consciously in the case of novel situations, or unconsciously in the case of familiar situations.

Human decision-making processes are heuristic in character; they are imprecise, fuzzy, and based on incomplete information. They contrast markedly with the general precision and completeness of algorithmically obtained, truths. The suggestion that conscious cognitive processes are essentially non-algorithmic may also bring forth an explanation, in part, for our ability to interpret non-literal expressions. Non-literal expressions rely, not on definition, but on the variations of sense that arise from contextual nuances and the inherent patterns of language structure. At face value, it seems that the creation and interpretation of non-literal expressions is not amenable to algorithmic processes. Neither, is our ability to deal with antinomy and paradox, or our ability to laugh when we encounter comedy or coincidental cases of similarity. Perhaps cases such as these can only be dealt with by 
non-algorithmic processes. If consciousness does exceed that which can be achieved algorithmically, then it may follow that these sorts of problems may only be solved by conscious beings.

If algorithms exist in World 3 (the world of theories) and interact with World 2 (the subjective, folk-psychological world) to implement changes in World 1 (the physical world), then we are faced with the question of how those interactions occur. That is the major challenge for any theory of mind. If humans were able to function non-algorithmically, that would be consistent with the existence of non-algorithmic events in the universe, and in agreement with the weak anthropic principle. This principle says that the conditions for conscious life happen to be just right at our present time and place in the universe - simply because if they were not, we would not find ourselves to be in this place at this time (Penrose, 1989, p. 433).

\subsection{Conclusion}

The lack of predictive power of formal leadership theories is not a matter of finding a better formal theory. Leadership is simply not amenable to global generalisations in the guise of formal, algorithmic, rule-governed explanations. What has been argued in this chapter is that leadership is a phenomenal concept with phenomenal properties. Phenomenal qualities are perceived by their differences and are communicated both explicitly through propositional attitude sentences and implicitly through patterns of salience. It is for this reason that leadership means different things to different people, and is interpreted differently as situations and cultural milieus change.

The argument presented in this chapter may be summarised as follows:

1. Due to its intentional character, leadership is appropriately classified as a phenomenological concept of folk psychology.

2. The intentionality of leadership is narratively conveyed by means of propositional attitude sentences, which possess a logically ambiguous structure. 
3. Propositional attitude sentences are incomplete expressions of the information content of propositional attitudes, which also comprise the tacitly known information content of cognitive schemas.

4. The leadership concept resists theoretical description in general terms because of the logically incomplete and ambiguous information content of propositional attitudes.

5. Leadership cannot be separated from the problems of consciousness and freewill, which are related to our ability to construe meaning from ambiguous concepts and make decisions. Leadership is, therefore, not amenable to description in naturalistic, causal-computational terms.

Given this analysis, it does not seem possible to capture leadership in a formally structured theory, or to describe leadership in naturalistic terms. Hence, to talk of leadership in organisations will require thinking at the boundary of situational narrative and the grand narrative of formal theories.

The nexus of scientific and narrative accounts of leadership lie in human emotions; human emotions as described by the propositional attitudes of belief, intention and value; and human emotions as explained in terms of parallel distributed processing systems. In this and previous chapters, I have tried to show that the information content of human emotion may be conveyed narratively by means of propositional attitudes sentences. In Chapter 7, I will attempt to show that the information content of emotion may also be described as an emergent property of distributed cognition. 


\title{
Chapter 7: Distributed cognition
}

\begin{abstract}
In the previous chapter, it was argued that the physical representation of information through the causal-computational processes of distributed cognition and the 'raw feels' of phenomenology share the one information space of human experience. This chapter describes distributed cognition as a product of the causal-computational interactions of brain, body and world. The information inherent in the environment, culture and artefacts of organisations is both the antecedent and the product of human cognition. It is this naturalistic perspective of human experience that has most promise for explaining organisational functioning.
\end{abstract}

\subsection{Introduction}

In the previous chapter, I argued that leadership is not amenable to formal explanations, which employ naturalistic categories of cause and effect. In this chapter, I argue that organisations are amenable to such generalisations when those explanations cohere with distributed cognition theory. Given that phenomenal accounts of leadership and naturalistic accounts of organisations do not employ the same categories, explaining organisational functioning in terms of leadership poses a significant difficulty.

Halverston and Clifford (2006), omit the analysis of leadership altogether from a school case study analysis. Even though the interactions of a principal with teachers were examined, the focus of their study was on the cognitive system, 'aggregated networks of artefacts and actors necessary to complete complex organisational tasks' (Halverston and Clifford, 2006, p.585). This included attention to questions regarding the identity of the tasks under analysis, the artefacts relevant to the performance of that task, and the nature of the cognitive system. Whilst causalcomputational processes are important to such an analysis, the authors suggest that the free-will intentions of people and the internal stability of established sociocultural structures are not amenable to analysis under distributed cognition theory. 
The phenomenal experience of leadership persists in the narrative accounts of folk psychology. Yet, organisational functioning can be satisfactorily explained by the naturalistic account of distributed cognition. Building on the argument given in Chapter 6, I put a case for phenomenal accounts of leadership being placed alongside naturalistic accounts of organisational functioning. This argument relies on the assumption that both phenomenal accounts and naturalistic accounts share a single information space.

From an objective, outside-looking-in perspective of human life, phenomenal accounts are consequences of the environmental, cultural and cognitive processes of distributed cognition; whilst from a subjective, inside-looking-out perspective, they are manifestations of subjective feels, or qualia. Hence, the phenomenal and naturalistic accounts of human life examine the one information space from different standpoints. Given our growing knowledge of the part of emotion in human cognition and the resilience of emotionally charged intentional categories in the narratives of folk psychology, human emotional experience provides a convenient focus for a dualistic analysis of organisational life: One that employs a naturalistic account of organisational functioning alongside a phenomenological account of leadership.

Distributed cognition theory can contribute to a naturalistic coherentist account of organisational functioning by explaining the interdependent character of human learning and action. According to this theory, cognition occurs through the dynamic interaction of mind, body, and world, where world comprises artefacts, culture and environment.

\subsection{Mind}

Clark and Chalmers (2002, p. 643) ask the question, 'Where does the mind stop and the rest of the world begin?' A theory of mind that limits human cognition to the boundaries of skin and skull is inadequate to the tasks of explaining the functioning of organisations. Insofar as we use our environment to store memories in notebooks, magnetic storage media, photo albums and suchlike, our minds extend beyond our 
skulls. However, we also utilise our environment to process information and represent knowledge. This occurs, for example, whenever an accountant out-sources a calculation task to a handheld calculator; when directors, actors, film crew and others collaborate to produce a documentary; or a group of teachers adapt their teaching methods to suit the geometry of an open-plan teaching space.

The human mind and the brain are sometimes taken to be synonymous, even to the extent that mind and brain are considered identical. There seems to be little doubt that the human brain is, at least, a requirement for mind. The brain is the body region where significant cognitive processing takes place, including the conscious and unconscious processing associated with decision-making.

\subsubsection{The routine tactical moves of leaders}

The cognitive processes of decision-making cannot be a purely conscious affair. In stressful, time-poor moments of organisational life, decisions tend to be taken according to established patterns of behaviour. Routine responses are those that have been rehearsed on repeated occasions; they constitute responses that are nonconscious and automatic rather than conscious and rational. Over time, these routine responses may be reinforced by successes or incapacitated by failures. The complete repertoire of automatic responses acquired by a leader through experience provides him or her with a selection of rehearsed tactical moves, each one associated with a particular type of situation. All that is required is that, within each episode of a problem situation, a tactical move is enacted - and that may be a matter of unconscious habit rather than conscious decision.

Certain sorts of habits may be promoted by organisationally sanctioned procedures. However, as Wilson (1988) explains in his analysis of pilot habits in airline accident prevention, the most frequently repeated actions are the ones most likely to become habits:

There is no such thing as not using a procedure, if you disregard one you use another in its place. Whichever one is used the most is the one that will likely become habitual. If the safer and more professional procedure 
is not your habit, something less will be, and that opens the door to error (Wilson, 1988, p. 2).

The tendency to fall back on habitual responses is shared by leaders and nonleaders alike. The distinction between the two has much to do with the formal roles and functions of leaders in organisations and phenomenal perceptions of leadership capability. All people adapt to changing situations, at least in part, by employing rehearsed habits of behaviour. Routine tactical moves provide a stable base of practical knowledge that may assist the resolution of a problem. This might occur if an automatic response reduces the leader's cognitive load so that he or she can focus cognitive resources on a problem's unique features. However, at other times an automatic response may constitute an inappropriate response to a problem. This might occur, for example, when the perceived similarity of the situation at hand is due more to its surface features rather than its deeper characteristics.

Situation types that have been repeatedly encountered require less conscious deliberation than situations that are novel or unique in a person's experience. Routine or habitual responses, established through experience, are cognitively economical: they simply require that a situation be classified, then that the matching routine be initiated and enacted automatically. These automatic processes require a minimum of attention, and free up cognitive resources for conscious deliberation. They also enable prompt action to be taken.

With deliberation that is more conscious, routine responses may also be adapted to fit a situation that is only partly similar to those previously encountered. However, adapting an existing tactical move or creating an entirely new one places a larger cognitive load on the leader and requires access to additional resources and more time to fashion. If an adapted or entirely new response succeeds then it may in time become a new routine, if not it is likely to be avoided or forgotten.

Another way that a routine response could arise in a designated leader is by the social transmission of routines from person to person; that is, by a form of cultural transmission. Hence, as leaders declare or demonstrate successful strategies to one 
another, seek aid from one another, or share resources, the mutual influence of a network or community of leaders may bring about a socially preferred set of routines. Similar sorts of processes may arise as the formal leader interacts with others, including subordinates, in an organisation. Such processes may be attributes of distributed leadership.

Socially distributed processes may also give rise to culturally accepted routines within an organisation - processes to which little conscious deliberation is given because they have been used successfully in the past. The process of establishing such routines within an organisation might be labelled as cultural leadership. However, when leadership is cast in terms of the causal efficacy of individuals, who have been labelled 'leaders', then culture is limited to the alignment the intentionality of organisational members with the intentionality of the leader. This conception of culture is one that is governed by a central executive agent. Such a view fails to take into account the dynamic interactions of organisational members with one another, with people outside the organisation, with the theories and artefacts in use, and with the physical environments in which people work.

Explaining leadership as the outcome of cultural processes requires that culture be explained. However, attempts to separate culturally mediated leadership processes from other types of culturally mediated actions have had a notable lack of success. For example, trait theories of leadership have failed to identify, in general terms, the characteristics of leaders that distinguish them from other people. Traits, attitudes or temperaments are stable dispositions that are attributed to individuals. Traits include those exhibited in the 'darker side' of leader-follower relations as highlighted by Clements and Washbush (1999), and those that 'animate, motivate, and direct our abilities toward good and productive thinking' (Ritchhart, 2002, p. 21).

Although some traits might be described as inherited dispositions, it is much more plausible to explain them as culturally mediated characteristics, because they obtain their meaning by reference to culture. Trait theories of leadership rely on the assumption that each leader or follower possesses dispositions that remain stable regardless of the situations in which they work. However, the meaning of observed 
traits depends upon their semantic relations to the content of situations. Like all other observations, trait attributions are theoretically constrained interpretations of complex interpersonal interactions. Consequently, the characteristics of particular traits are socially mediated so that they depend on the significance attributed to them according to culture and the circumstances in which they are observed. The difficulty with trait theory is that the traits attributed to leaders may be exhibited by other people. It is not clear how those traits can be indicators of leadership. Trait theories claim that specific personality types are more adept at analysing organisational needs, organising appropriate action and motivating others to take those actions, regardless of the situation under consideration.

\subsubsection{Leadership and the meaning of phenomenal experience}

Transformational leadership theories also speak of a leader's ability to motivate others to "do more than they originally intended and often more than they thought possible' (Bass and Avolio, 1994, p. 3). The inclusion of motivational dispositions and goal directed action in theories of leadership is an attempt to explain concerted social action by explaining how people become collectively motivated to act in particular ways. Free-will agents must be motivated in some way if they are to choose to follow, rather than to ignore or resist, a leader's initiatives. If people were to act in a concerted way simply because they were physically compelled to, or were so disposed to certain courses of action that they could not choose otherwise, then that concerted action would not normally be considered leadership as portrayed in the narratives of folk psychology. In folk psychology, intentionality is a requirement for leadership. Leaders may choose to lead people along particular courses of action because they believe it is the best path to follow; and followers may choose to fulfil a leader's intentions because they trust the leader's judgement, have feelings of commitment towards the leader, or because they fear the consequences of not fulfilling the leader's instructions. Conversely, humans may act in a concerted way without coercion or compulsion, yet without any one person standing out as the leader of that concerted action. Conscious, free-will intentionality is a requirement for leadership, but does not entail leadership, and is not sufficient for leadership. Intentionality is a phenomenological property that entails being focussed on 
something (Smith, 2005). It stands in contrast to intentional irrealism, which challenges the existence and relevance of intentionality and assumes that human action can be explained in terms of physical properties alone (Stich, 1996, p. 168). Intentional irrealism is a position of extreme naturalism. If intentionality is irrelevant to a naturalistic account of organisational functioning, then it follows that leadership is also irrelevant to such an account. However, Stich (1996, p. 169) argues that the naturalisation of the intentional is vitally important. He considers it implausible that the ontology of social science (e.g. wars, phonemes, rituals, and hyper-inflation) should stand in some special relation to the ontology of the physical and biological sciences (e.g. quarks, molecules, genes, and chaotic systems). That is,

there is no single, special relation that all and only the properties invoked in respectable sciences bear to physical properties (Stich, 1996, p. 197).

As intentional categories are expressed in language, the meanings of the phrases used to express those categories rely on the semantic properties of primary and secondary intensions (here intentions, spelt with an ' $\mathrm{s}$ ', is distinguished from intentions, spelt with a ' $t$ '). Whereas intention refers to purposeful attention, intension refers to the semantic content, or sense, of a word. Intension is important to the analysis of propositional attitudes, which are themselves expressions of intentionality. The word 'leadership', can also be analysed according to its primary and secondary intensions. Here, I try to show that leadership is not equivalent to concerted group action, regardless of whether that concerted group action is conceived naturalistically or not.

\subsubsection{Can concerted group action constitute leadership?}

The interview responses of the four formally designated leaders at Eucalypt Primary School reveal a sense of connectedness between the principal and others and may imply sustained, concerted group action:

It's lovely to have a blend of people in your staff and also in your leadership team who have different ways of viewing [things]. Carolyn's very strategic, very organised, I'm probably seen as disorganised and my 
ideas are offbeat. I completely lose track; I can be thinking of lots of different things at the same time and recall them (Interview B5).

Whilst the Principal values the contributions and viewpoints of others, the School Council President recognises the importance of connectedness and the value of communication to problem-solving in the school:

I liaise fairly strongly with the principal. We communicate quite closely about the issues within the school that she thinks I need to know about, and I talk to parents about issues that they may want to speak to me about. If there is a problem, we try and get it resolved quickly to everybody's mutual agreement (or whatever) (Interview B6).

The Assistant Principal also expresses her sense of connectedness to a significant other. She attributes symbolic value to her mentor, so that the mentor becomes an archetype for good decision-making:

Often, when I'm confronted with issues and think, 'How am I going to react or deal with this situation?' I think of my mentor, she was a Leading Teacher [Level] 2 here, and is now principal at another school, and who is just fantastic. So, I guess I do often think of her: How would she react? How would she handle this situation? And I sometimes ring her. I think, because I am only in an acting position (this is all very new to me) that certainly when she was here, I relied on her heavily. Now that she's gone and I've jumped up, I still ring her; so I guess I'm still relying on her a bit (Interview B7).

Finally, Margaret, a leading teacher of Eucalypt Primary School understands that teacher knowledge does not reside in individuals, but is enacted through relationships between people:

I've worked closely with Eric and learned a lot from Eric because he's so very knowledgeable anyway. But, he's great to work with and I said to him last year, what I felt I needed was somebody else to work with me (being new and not knowing anything, and having, "Here it's all yours, your computer you must do all this"). Even though at the time it made me learn a lot, I felt in that area you definitely need some support. So I stayed with him to give him support this year (Interview B8).

Here, I have used the hermeneutic circle to interpret the responses of the subjects. Of course, the subjects may interpret each other's comments in ways that differ from 
mine. Similarly, other readers may view these statements from substantially different theoretical viewpoints and derive different meanings from them. Since a variety of meanings may be attached to the narratives, the question may be asked: "Are these really accounts of leadership?'

According to Chalmers (1996, p. 62), the meaning of a concept can arise from the primary and secondary intensions of linguistic reports, where the intension of a concept is a composite of attributes. These intensions constitute the a priori and $a$ posteriori aspects of meaning respectively, such that each intension attaches a particular kind of meaning to the concept. The primary and secondary truth conditions of a concept always yield truth in the actual world. They do not pose the risk of ambiguous meanings because their difference pertains to their application to possible worlds.

The primary intension of a term has epistemic content that yields a primary proposition applying only in those situations where it would turn out to be true. A primary proposition is necessarily true in an a priori sense because, its truth is independent of the context in which it is uttered. Hence, the statement, 'water is wet' is true in the set of worlds for which water is wet. If we possess the relevant concepts, we can know that the statement 'water is wet stuff' is true because the primary intensions of the terms 'water' and 'wet stuff' are the same.

In contrast to primary intensions, secondary intensions have subjunctive content that depends upon the context to which it refers (Chalmers, 2002). The secondary intensions of a statement yield a secondary proposition, which specifies the conditions required for truth in counterfactual (or imaginary, or other) worlds, given that the actual world turned out the way it did. For example, the secondary truth conditions of the statement 'water is wet' specify the conditions under which water is wet (i.e. it is in liquid form, it possesses surface tension, it is attracted to a hydrophilic surface, etc.). Since water is also $\mathrm{H}_{2} \mathrm{O}$, the statement also specifies the conditions under which $\mathrm{H}_{2} \mathrm{O}$ is wet. Whilst the primary intensions of 'water' and ' $\mathrm{H}_{2} \mathrm{O}$ ' differ, they possess the same secondary intension. 
When the secondary intensions, but not the primary intensions, of a statement support a necessary proposition, then a case arises of what Chalmers (1996, pp. 64, 146-149) calls Kripkean a posteriori necessity ${ }^{66}$. It is not necessary for water to be $\mathrm{H}_{2} \mathrm{O}$ because that would only apply in worlds where watery stuff has a very specific molecular structure. Hence, $\mathrm{H}_{2} \mathrm{O}$ does not logically supervene on watery stuff. Similarly, having a particular molecular structure does not logically supervene on wetness.

Extending this analysis to organisations and leadership, leadership cannot logically supervene on the enactment of concerted group action, which concerns the physical actions of organisational members. Concerted group action can conceivably occur without leadership. For example, when self-organisation occurs in nature, such as in the formation of the crystalline structure of a snow flake, a form of concerted group action occurs. The apparent cooperation and coordination of the water molecules occurs because the properties of water molecules cause them to interact in particular ways to bring about a systematic pattern. Concerted group action and leadership have different primary intensions, yet may have the same secondary intension. Consequently, the question of whether a particular instance of concerted group action is also an instance of leadership depends on the a posteriori circumstances that brought about that instance.

\subsubsection{Folk psychology}

As illustrated in Chapter 4, the leadership narratives of folk psychology do not rely on a naturalistic analysis of organisational action to convey the concept of leadership to the reader. Folk psychology contrasts with science because it is not the product of systematic study and critique, but arises from the intuitive, phenomenal statements people make about themselves and others. These intuitive statements include the subjective explanations, predictions and descriptions people offer about the mental states and intentions of others (Davies and Stone, 1995, p. 1).

\footnotetext{
${ }^{66}$ Kripke's (1972) critique of identity theory is an argument against materialism due to the failure of logical supervenience.
} 
Since leadership is also explained in terms of the beliefs, intentions and actions of people, it qualifies as a concept of folk psychology. There are two dominant folk psychological theories of mind, the theory-theory and simulation-theory. Other, more specific theories of folk psychology include eliminative materialism, epiphenomenalism, identity theory, interactionism, naturalistic functionalism, and parallelism. These theories are subjects of intense philosophical and scientific debate.

The narratives of folk psychology infer or declare law-like generalisations about leadership, and couch those inferences and generalisations in intentional terms (Stich, 1996). However, according to the Twin Earth argument ${ }^{67}$, content cannot supervene on non-relational physical properties. Folk psychology attributes meaning to content in a holistic manner such that two beliefs can only be identical if they obtain meaning from similar contexts or networks of doxastic surrounds. This poses a problem for folk psychology if an extreme version of holism is adopted, for identical doxastic surrounds would be required for two beliefs to be identical. That is, it would not be possible to generalise a single belief to a variety of other contexts according to similarity; extreme holism requires much more than similarity, it requires identity. Since the goal of naturalism is to obtain generalisations, it stands in opposition to extreme folk psychology (holistically construed). The broad generalisations of folk psychology seem likely to conflict with the nomological generalisations of future cognitive science, such that there will be no true content based, folk-psychological generalisations to be found (Stich, 1996, pp. 22-27). If the intentional or semantic content of folk psychology cannot be naturalised in terms of causal relations, it follows that it will not be possible to reduce causal relations to non-intentional, non-semantic properties. Intentional content cannot be described in terms of physical properties.

Folk psychological capacities include our ability to describe each other and our behaviours, make predictions, and anticipate the behaviour of others. They also include the ability to pronounce broad generalisations that other people will readily

\footnotetext{
${ }^{67}$ The Twin Earth thought experiment, attributed to Putnam (1975/1985), is put in support of the argument that meaning depends partly or wholly on factors external to the speaker.
} 
accept or endorse such as, for example, 'if a child wants a lolly and a lolly is offered to her by her mother, then typically she will accept the lolly'.

Eliminative materialism, however, claims that folk psychology is a seriously mistaken commonsense theory. Folk psychology is expressed through the common knowledge of ordinary people. This knowledge, whilst being consciously accessible, arises from a rich experience of tacitly or subconsciously known generalisations, which are also shared within culture. On one view, these tacit generalisations arise from an internally represented grammar, which also figures in the production of speech. Hence, folk psychology is understood to be analogous to a set of linguistic rules that contribute to the production of propositional attitudes (Stich, 1996, p. 128).

The argument for the elimination of folk psychology from naturalistic accounts of human cognition hinges on the view that the propositional attitudes, at the core of folk psychological thought, arise from linguistic or sentential cognitive processes. However, a naturalistic account of human cognition refers to the cognitive machinery of the brain, which is understood to function according to patterns of electrochemical activation amongst vastly interconnected neurons. Grammatical rules do not fit such an explanation. Indeed, it is difficult to see how electrochemical processes of themselves can give rise to a common grammar that underlies all human thought. Given the variety of grammars in use in the languages of the world, it seems likely that the emergence of grammar depends upon human interaction and communication. The electrochemical processes of the brain perform computational functions, which are the cognitive processes are processes that involve the conversion of vectors of neuron activation from one configuration to the next. Hence, P. M. Churchland (1995) says,

It is now fairly clear that the basic unit of computation is the vector-tovector transformation. And it is now evident that the basic unit of memory is the synaptic weight configuration. None of these things have anything essential to do with sentences or propositions, or with inferential relations between them. Our traditional language-centred conception of cognition is now confronted with a very different brain-centred conception, one that assigns language no fundamental role at all (Churchland, 1995, p. 322-323). 
Churchland's eliminative materialism questions the causal efficacy of beliefs, desires and many other mental states. If this view is correct, then leadership, a concept that depends on the expression of beliefs, desires, and other mental states, also lacks causal efficacy. Eliminative materialism typically relies on two premises; the first being that beliefs, desires and other mental states are theoretical terms of folk psychology; the second being that folk psychology is seriously mistaken because some of its central claims about the sentential states and processes that give rise to behaviour are false or incoherent. From these premises, the eliminativist argument concludes that the very existence of propositional attitudes, as causal cognitive states, can be questioned (Stich, 1996, p. 4).

If propositional attitudes are understood as rational, sentential objects, then they have the character of theory. However, if they are understood as the 'raw feels' or qualia of phenomenal experience, then they take on a semantic character, more akin to the holistic mechanisms of emotion than to sententially expressed reason. The way we view leadership, as a concept of folk psychology, depends partly on whether we understand folk psychology to be either a sentential theory or an internal process by which we simulate the thoughts of others. These are the views presented by the theory-theory of folk psychology and the simulation theory of folk psychology respectively. A third view blends the two folk psychology theories together into a single package. The argument against folk psychology, is an argument against the theory-theory versions, and loses much of its force if folk psychology is constituted by off-line simulation, completely or in part.

Theory theories depend on perceptually-dependent processes or the on-line processing of information to explain, predict or describe events. Simulation theories, however, posit our ability to imagine or empathise with the thoughts and actions of others. Simulation theories differ from theory theories in that they posit reasoning processes that are divorced from perception as off-line mental processes. According to simulation theory, folk psychology is not a theory at all, simply the capacity to imagine ourselves in the situation of another person and to simulate the other person's thinking. Hence, cognitive simulation has a predictive function that informs practical reasoning and action (Gordon, 1995, p. 71). Theory-theory could plausibly 
enable predictions to be made. However, simulation-theory enables internally experienced emotional responses to be simulated, whilst theory-theory does not seem to provide for this possibility.

The narrative accounts of folk psychology do enable emotional experiences to be conveyed, or felt, by means of literal and non-literal language forms. We possess common beliefs and desires about the world and its organisation, which include the concept of personhood and the desire to maintain coherence in our world views. According to Bruner, 1990, p. 39) the narratives of folk psychology are created in response to violations of our constituent beliefs. They enable these violations to be resolved through the creation of worlds outside ourselves, which consequentially serve to reinforce or alter the expression of our beliefs. Hence, an inner world of experience interacts with an outer world of narrative, giving rise to a subtle drama of human action and narrative structure. Meaning has a central place in this narrative structure:

\begin{abstract}
I believe that we shall be able to interpret meanings and meaning-making in a principled manner only in the degree to which we are able to specify the structure and coherence of the larger contexts in which specific meanings are created and transmitted... It simply will not do to reject the theoretical centrality of meaning for psychology on the grounds that it is "vague". Its vagueness was in the eye of yesterday's formalistic logician. We are beyond that now' (Bruner, 1990, pp. 64-65)
\end{abstract}

The vagueness of emotional responses, and of non-literal meanings, are akin to phenomenal experience; and leadership shares more in common with phenomenal experience than causal physical processes.

\title{
7.2.5 A computational model of mind
}

Despite the phenomenal character of leadership, one of the claims of traditional theories is that leadership has causal properties. The proposition that leaders bring about observable events in organisations relies on two significant assumptions. Firstly, that it is leadership - not some other type of cause - that brings about a particular sort of event. Secondly, that leadership is a product of the mind of the leader. Whilst a theory of distributed leadership may attribute leadership to the 
collective actions of people, there is a difficulty in distinguishing leadership acts from non-leadership acts. Distributed leadership theory also needs to explain how the collective intentionality required for leadership comes about.

To talk of distribution of leadership requires that the phenomenal qualities of leadership stand alongside the naturalistic properties of distributed cognition theory, which explains cognition in terms of the causal-computational interactions of mind, body and world. Whilst distributed cognition theory is able to describe how information processing can be distributed across a computational system, it is not clear that any phenomenal understanding of leadership can be explained by such a theory.

Whilst I accept that distributed cognition theory can provide a valid and theoretically feasible account of human cognition, I am not convinced that a naturalistic account alone can capture all there is to human experience. If we understand mind to be a system that produces high level thinking, or at least a high level of information processing, it may seem convenient to conceptualise mind as a kind of 'software' running within the 'hardware' constituted by the brain. This is how a symbolic serial sentence program is implemented and some researchers think this type of system is a sufficient explanation for mind. For example, Simon (1996, p. 100) argues that serial computers have been thinking logically, creatively and intuitively for the last half century. Others conceptualise mind as a parallel distributed computational system in which the distinction between hardware and software is blurred and the system dynamically reconfigures itself in response to internal and external feedback loops. This sort of system can be described mathematically as activation vectors enacted by a system of nodes (or neurons) connected in parallel. Lehrer (1996) argues that conscious mental activity can be realised through the activation vectors of a parallel distributed processing machine. Hence, mind may be portrayed as the implementation of serial programs within serial-sentence architecture; or as vector to vector transformations performed within parallel distributed architecture. 


\subsubsection{Vector to vector transformations}

In mathematics, a vector is a set of numbers organised according to a prescribed convention. Vectors are a convenient way of representing the activity levels of a set of neurons such that the activity of each individual neuron is related to the activity of the other neurons. If a set of neurons is considered here as an example, their activation could be represented as $[0,1,1,0,0,1,0]$ in one situation and $[0,1,1,1$, $1,0,1]$ in another. The variations between the two vectors represent the different patterns of activation brought about by the given situations.

The activation patterns shown by vector coding represent the activation of each of the nodes in a network such as that shown in Figure 7.1. In this example, a network configuration is shown for an artificial neural network designed to solve the standard exclusive-OR problem. Here, the network is required to give an output when a given input is provided by one, but not both, of two sources. In this case, the network is designed as a parallel distributed processing system, but is simulated within a symbolic serial system (a standard personal computer). The network design includes two input nodes, two nodes in a 'hidden layer', and a single output node. When each hidden and output node receives an input above a given threshold, it produces an output determined by a non-linear sigmoid function. Bias inputs are introduced to the hidden and output layers to improve the learning capability of the network. The structural design of the network determines whether the network can solve particular types of problem. In this case, to be able to solve the XOR problem correctly, the network requires a hidden layer as well as the input and output layers. To correctly solve the XOR problem, when the input ' 1 ' is given by one, but not both of the input nodes, the output node of the network should return ' 1 '. 


\section{Figure 7.1 Parallel distributed network design: XOR Problem ${ }^{68}$}

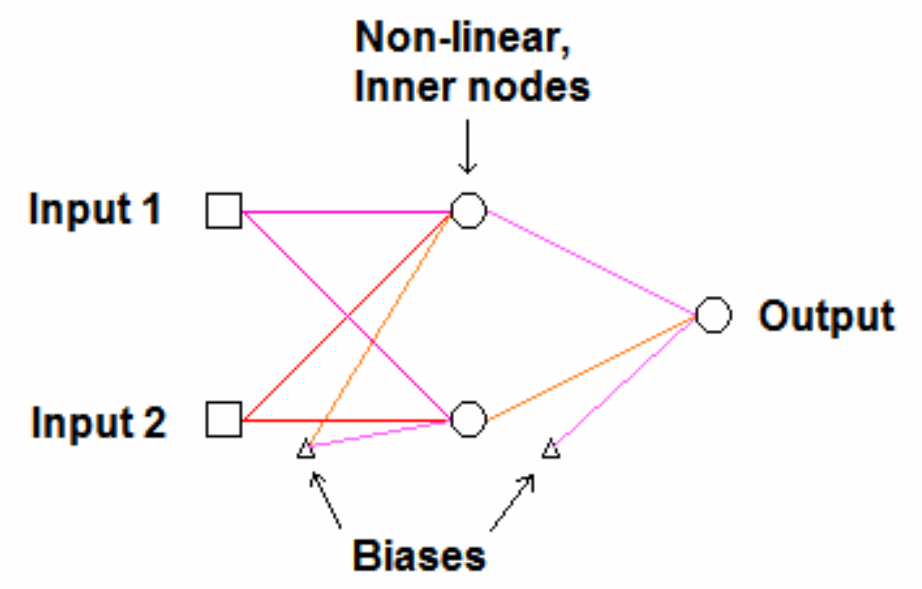

The network is trained with a set of training data, which gives the correct outputs for each of the four possible inputs. With each training cycle, the computation performed by the network is checked against the training data and the activation weights adjusted according to a weight change algorithm, based on the learning process proposed by Hebb (1949). This process mimics the electrochemical activation processes of the neurons in a biological nervous system.

Table 7.1 shows the inputs and outputs of the network after training had been completed. When inputs of ' 0 ' and ' 0 ' are given, the output activation value is 0.01 , which is below the activation threshold for the output node. Consequently, the output is given as ' 0 '. The computation performed by the network (e.g. from 0,0 to 0 ) is a vector to vector transformation.

Table 7.1 Input, output and target data for the XOR problem

\begin{tabular}{|c|c|c|c|}
\hline Input 1 & Input 2 & $\begin{array}{c}\text { Output } \\
\text { Activation } \\
\text { value }\end{array}$ & Output \\
\hline 0 & 0 & 0.01 & 0.00 \\
\hline 0 & 1 & 0.99 & 1.00 \\
\hline 1 & 0 & 0.99 & 1.00 \\
\hline 1 & 1 & 0.01 & 0.00 \\
\hline
\end{tabular}

${ }^{68}$ This network diagram was adapted from that provided in the artificial neural network simulator, Quiknet32, designed by Craig Jensen, 1997. 
The inputs and outputs of the network can be portrayed as a three-dimensional surface plot as shown in Figure 7.2. This diagram shows that when the inputs are simultaneously low or high, they return a low value in the output.

\section{Figure 7.2 XOR surface plot (after training) ${ }^{69}$}

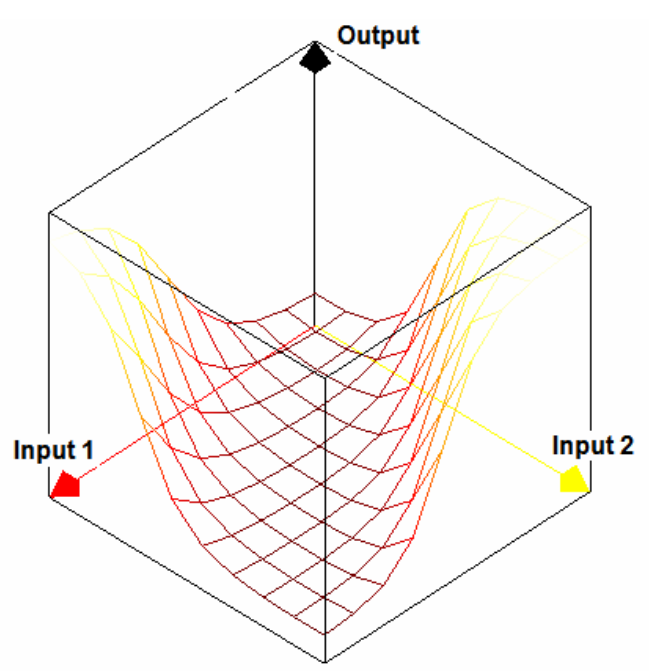

If the surface plot is produced during the early stages of planning, as in Figure 7.3, the absence of an orderly and coherent plot is evident. At this stage of the training, the network has not yet 'learnt' to solve the XOR problem and the percentage of correct outputs is zero.

${ }^{69}$ Produced using Quiknet32, designed by Craig Jensen, 1997. 
Figure 7.3 XOR surface plot (in the early stages of training)

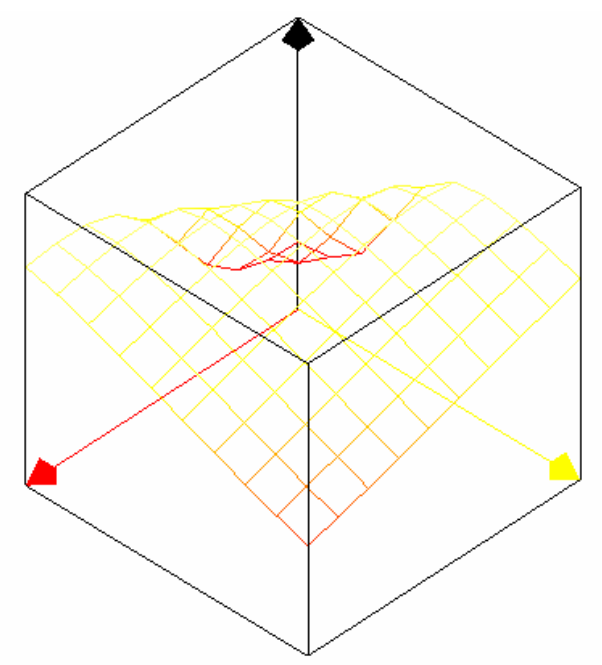

With further training, the network produces a plot that more closely resembles the plot shown in Figure 7.2. Neural networks function recursively so that networks with an appropriate structure can be trained to solve particular kinds of problems. In living creatures, appropriate networks may have arisen through processes of natural selection. Networks that can solve particular kinds of problems survive and are replicated. Networks that cannot solve those problems do not survive.

Artificial neural networks are examples of parallel distributed processing systems that can 'learn' as they adapt to environmental inputs. Whilst artificial neural networks are often described as being more robust and flexible than computing systems built on serial architectures, they can be simulated on symbolic serial sentence systems. The difference between the local coding of symbolic systems and the vector coding of distributed systems, as Churchland and Sejnowski (1992, p. 163164) explain, is a matter of perspective. However, in many practical applications, distributed architectures have performed well, particularly when fuzzy, dynamic or robust systems are required.

70 ibid. 


\subsubsection{The limitations of computational systems}

Both symbolic serial sentence processing and parallel distributed processing architectures provide useful architectures for the mechanisation of problem-solving processes. Many applications of these architectures are presently available for educational, production, entertainment and military purposes, just to name a few. Computational machines are clearly expanding the reach of human cognition, enabling us to store vast amounts of information and solve complex computational problems quickly and easily. The reach and computational power of these machines seems likely to increase far into the foreseeable future.

Computational machines operate according to rules or algorithms, which can only be modified by human intervention, or dynamically modified by the computational system in response to external inputs. However, Lucas (1996) argues from Gödel's undecideability theorem that machines cannot do all the things minds can do and reasoning cannot be entirely rule bound. With respect to a sequential symbol processing system, he says, rational minds are able to transcend the rules of any logical system and 'construe the Gödelian well-formed formula not just as a string of symbols but as a proposition which is true' (Lucas, 1996, p. 103). A sequential symbol processing system is one that stores data in local memory addresses; it differs in significant ways from a parallel distributed processing system, which distributes data across the system. Clark (1989) explains that the

distinction between a local and a distributed representational system is somewhat perspectival. Distribution is in the eye of the beholder or at best in the functional requirements of the system itself (Clark, 1989, p. 95).

Furthermore, it is possible, in principle and in many practical cases, to simulate a parallel distributed processing system within a sequential symbol processing system (Clark, 1989, p. 121; Wells, 1993, p. 538; Ricciulli et al., 1995).

The argument for the simulation of parallel distributed processors on serial symbol processing machines is not without detractors. Lyngzeidetson (1990) argues that a parallel distributed processing system is able to subvert the requirements of a 
formal system by constructing its own semantic metalanguage. This occurs, he says, whilst the computation is in progress, by the dynamic reorganisation of 'the active data structures' of a computation (Lyngzeidetson, 1990, p. 6). Lyngzeidetson further argues that the in-principle subversion of Gödel's undecideability theorem becomes possible in a parallel distributed processing system through the morphing of the rules of the formal system. However, Lyngzeidetson's argument is countered by Wells (1993), who argues that

the dynamic construction of structures of various kinds in memory is commonplace in serial computers. Languages as diverse as Common Lisp, Pascal and Snobol include dynamic storage allocation in serial implementations (Wells, 1993, p. 538).

Both serial symbolic and parallel distributed processing systems possess active structures that enable them to adjust the way they process information. In both cases, the data structures can be dynamically reconfigured to meet the particular demands of a computation. If Lyngzeidetson's conclusion was accepted at face value, then it would follow that serial computers could also subvert Gödel's theorem. Such a conclusion is clearly false; it stands in clear contradiction to Gödel's mathematical proof and is therefore incoherent.

Lucas (1996) argues that a finite computer, acting according to specific deterministic system of rules, is unable to prove the Gödel formula of the system it employs. Such a system will consist of a formal logistic calculus, a large finite number of well-formed formulae and a finite, but smaller, set of rules of inference. A computer is unable to transcend the logical rules that determine its operation. Decidability in any formal system (or machine) requires that the system be complete, and from Gödel's theorem, a complete formal system will always be inconsistent (Kennedy, 2007). Hence, we are unable to put a rational case that we are machines. Following Lucas' (1996) argument, a deterministic machine, will never be able to do all that humans can do. Humans, however, are able to subvert the practical implications of Gödel's theorem. To argue that we are simply serial sentence or parallel distributed processing machines presupposes the consistency and decidability requirements of rationality. If we were machines, then our arguments 
would be inconsistent and therefore irrational. We are able to determine the truth of a statement in ways that a machine cannot.

The limitations of logical argument, and the computations that depend upon logical languages and mathematics, contrast with the apparent ability of humans to communicate ideas in non-literal ways and deal with problems that computers cannot. Inspiration, creativity, humour, irony and satire all draw on human abilities that go beyond that which can be achieved through literal descriptions or logical argument alone. Narratives of this kind derive their meaning from the contextual associations of emotion-related words and phrases. Emotion has semantic value because it arises in a response to the contextual cues of the situations of human experience. The meanings of emotion words are strongly influenced by culture (Heelas, 1996). Explaining human motivation and decision-making requires that reference be made to emotional experience, which arises from the interaction of mind, body and world.

\subsection{Body}

The literal and non-literal expressions of narrative evoke emotional responses such as laughter, melancholy, surprise, hope or aversion. Emotion has semantic relevance in focussing attention, evoking rapid responses to danger, and classifying the information available to us according to its relevance to our survival, well-being and prosperity. Emotion is a whole body phenomenon involving the complex processing of sensory information and information from memory. Emotion is relevant to the study of leadership because of its importance to human motivation and decision-making.

Emotions are important to the regulation of complex organisms at the level of homeostatic regulation. They also enable the mechanisms of life regulation, essential to the survival of organisms, to function. Emotions provide the link between basic life functions and high level reason and play an important part in the values we adopt as part of our autobiographical experience. It is impossible to separate emotions from ideas of pleasure or pain, freedom or compulsion, reward or punishment, or good or 
evil. Emotions are a necessary part of our decision-making capability; and they appear to be necessary for consciousness.

Damasio (2000) believes that consciousness, evoked as a stream of 'images', has its roots in our emotions. Here, he does not necessarily mean visual images; but the mental patterns of objects, which are related in some way to the physical distribution of information across neurons. This stream of images produces a wordless narrative of the autobiographical self:

I do not mean narrative or story in the sense of putting together words or signs in phrases and sentences. I do mean telling a narrative or story in the sense of creating a non-languaged map of logically related events. Better to think of film (although film does not give the perfect idea, either) or of mime (Damasio, 2000, pp. 184-185).

Hence, the words and sentences of language are translated from the non-linguistic images that stand for objects in memory. Those objects symbolise 'entities, events, relationships and inferences' (Damasio, 2000, p. 107). Understanding narrative in this way opens the way to understanding how an organism may possess consciousness without also possessing a language through which to express that consciousness. It also opens the way to understanding how language may have evolved, or how non-literal language may evoke meanings that differ markedly from the literal meanings of words or sentences. The autonomous survival of an organism depends strongly on the bodily mechanisms of emotion. Whilst, language plays a part in survival and consciousness in humans, it is not essential to consciousness, as may be seen in people who have lost the ability to communicate with language (see Damasio, 2000, pp. 108-112). 


\subsubsection{The Somatic Marker Hypothesis}

Damasio (1994) argues that emotion is a component of the internal mechanisms that bring about intelligent behaviour ${ }^{71}$. An important and perhaps the only functional characteristic of such behaviour is the act of making decisions that can be rationally justified. Damasio (1994) proposed his somatic marker hypothesis as a means of explaining the role played by emotion in the cognitive mechanisms of decision making. This hypothesis proposes that humans access non-conscious somatic information as well as consciously accessible information when making decisions.

Traditionally, a decision is a response to the set of possibilities presented by a given situation. A response could be a non-verbal action, a linguistic expression, or a combination of the two. The terms typically used to describe reasoning imply that a person making a decision possesses knowledge. Consequently, in a given situation, the decision-maker

- notices that the situation at hand requires a decision to be made;

- understands that the decision involves a choice of two or more alternative pathways of action; and

- possesses beliefs about the possible consequences of each of the possible pathways.

Here, the terms 'reasoning' and 'deciding' imply that a number of conscious cognitive processes are utilised in decision-making; specifically that attention is focused on the problems posed by the situation, that a logical strategy of inference is

\footnotetext{
${ }^{71}$ This is not, however, a reference to the rather nebulous concept of emotional intelligence that has gained popularity in popular and academic circles over the past decade. The concept of emotional intelligence largely grew out of the concept of social intelligence, and exhibits similarities to Gardner's (1983) multiple intelligences theory and the concept of intrapersonal and interpersonal intelligences. Salovey and Mayer (1990) describe emotional intelligence as the ability to regulate the emotions of self and to make decisions according to the feelings and emotions of self and others. However, emotional intelligence is also framed as a cognitive style held by individuals and exhibited as specific skills and traits. Waterhouse (2006) takes a strong position in her critical review of emotional intelligence theory and the related theories of multiple intelligences theory and the Mozart effect. She argues that these theories lack empirical support and theoretical power, and that they are harmfully misleading.
} 
in use, and that an appropriate working memory and resources for action are available. However, Damasio (1994) cites clinical and experimental evidence suggesting that this cannot give a full account of decision-making. In particular, he provides three examples of situations requiring decisions that cannot be fully accounted for in terms of the hypothetical, logical, and inferential approach described above (Damasio, 1994, pp. 166-167).

The first example concerns the simple process of a person deciding to eat. In this case, physiological 'know-how' is brought into play as the neurons in the hypothalamus respond to a drop in blood sugar. Here, there is no hint of a conscious process of analysis and inference; no awareness of the neural and biochemical processes leading up to the time when the person becomes aware of being hungry. In the second example, the situation of a person ducking to avoid a falling object is considered. Unless a conscious intervention is made to subvert the normal response to this sort of situation, the ducking response occurs automatically. Originally, the decision to duck may have been a conscious one; made in childhood after realising that falling objects can inflict pain or harm. Over time, though, an automatic stimulus-response has become instituted in the person's neural and sensory mechanisms so that the response occurs without any conscious intervention.

The third example is a cluster of more complex scenarios such as choosing a career, deciding what changes to make to a school curriculum, or attempting to resolve a conflict between two people. In these sorts of cases, logical inference may play an important part in the decision-making process. However, the inherent complexities in these examples also pose the problem of 'computational explosion'; that is, the number of inferential calculations needed, increase exponentially as the number of variables increase. This militates against the systematic and complete identification of possibilities and inhibits the adoption of reliable predictions. This third example differs from the first two because the types of scenarios it presents can be distant or close to the immediate interests of the decision-maker. For example, the decisions of a school principal, concerning the implementation of an administrative procedure that has little direct bearing on her school, is likely to be more distant from her personal and social domain than deciding to go to the side of her daughter, who 
has been badly hurt in an accident. The relative proximity of a decision to the personal and social domain cannot be factored into the traditional view of decisionmaking. What this view leaves out is the crucial role of emotions.

Damasio's (1994, pp. 173-177) somatic marker hypothesis proposes that humans refer to a somatic (or body) state that marks a particular sort of cognitive image called a 'marker'. This marker is experienced as a fleetingly pleasant or unpleasant 'gut feeling' which may include both visceral (e.g. quickened heartbeat) and nonvisceral (e.g. muscle tightening) sensations. The signal given by a marker may trigger immediate rejection of particular negatively framed courses of action, or the acceptance of positively framed ones. Either way, the somatic marker reduces the alternatives for action to a more manageable number. Consequently, a conscious, rational consideration of the remaining options becomes possible within a practically feasible period. Damasio suggests that somatic markers not only reduce decisionmaking to a more manageable process, but also enable better decisions to be made.

Somatic markers arise from the feelings generated by secondary emotions, which are the emotions that arise from experience. The primary emotions are innate physiological responses to particular stimuli that depend on the functions of the amygdala (two almond shaped regions, which form part of the limbic system). Since the secondary emotions depend upon the primary emotions, damage to the amygdala also affects decision-making ability. The secondary emotions arise from learned associations between particular categories of objects and actions on the one hand, and the primary emotions on the other (Damasio, 1994, pp. 131-150). Somatic markers are unconscious processes that assist conscious, deliberate decision-making. 
They are learnt from experiences that may have once been conscious and deliberate, but which over time have become automatic, unconscious responses ${ }^{72}$.

The view that 'gut feelings' play an important part in decision-making may be found in the narratives of folk-psychology. In her novel, Girl with a Pearl Earring, Chevalier (2000, pp. 132-136) writes of a maid, Griet, and her decision to change a scene her master, an artist, had created for his latest painting. Griet is bothered by the scene, feeling that she wants to change something but not knowing what. She has never tampered with her master's work before and to do so is a great risk to her livelihood. After considering the scene, she comes to a decision about what should be done and waits for her master to perceive it also, without her intervention. Whilst Griet waits for this to occur, she feels her chest grow tighter and tighter. This physical sign of emotion precedes her decision to take bold action and make the change herself. After doing so, she waits anxiously for him to see the change she has made to the scene. Her agitation is clearly apparent to her companion who says, 'You're acting like a chicken that know's its for the slaughter'.

After her master discovers the change, he curiously asks her why she had done this. She replies in terms of the phenomenological differences inherent in the intangible qualities of order and disorder; and in terms of a phenomenological complex, a composite of that which teases and pleases:

\begin{abstract}
"There needs to be some disorder in the scene, to contrast with her tranquillity" I explained. "Something to tease the eye. And yet it must be something pleasing to the eye as well, and it is, because the cloth and her arm are in a similar position". There was a long pause. He was gazing at the table. I waited, wiping my hands against my apron. "I had not thought I would learn something from a maid", he said at last (Chevalier, 2000, pp. 135-136).
\end{abstract}

\footnotetext{
72 The somatic marker hypothesis continues to be a subject of research and debate turning on differing theoretical distinctions between rational versus non-rational decision-making (Bechara et al, 2005). Maia and McClelland (2004) challenge Damasio's hypothesis, citing laboratory evidence that nonconscious somatic markers are not necessary. Their evidence is based on empirical analysis of strategies employed by subjects in a simple card game. However, they base their conclusions on the implausible assumption that that this simple case mimics the complexities of "real life" decisionmaking.
} 
Here, Griet expresses the propositional attitudes of being teased and pleased, and of surprise and acquiescence. Yet these attitudes arise in circumstances that include physical manifestations of emotion, which include tight chest and agitation. Griet is able to give a rational explanation for her decision - a demonstration of her conscious thinking; and displays secondary emotions in her cautious retention of tradition and social mores - a display of learned emotional experience. Yet, this is only possible because master and maid share an appreciation of art that is transcendental in character. This fictional account, therefore, retains a narrative structure, which is comprehensible because it conveys recognisable phenomenological concepts and emotional schemas.

\subsubsection{Emotion and value}

Conscious decision-making requires judgements about the value of alternative options for action, and those judgements are aided by non-conscious somatic markers established through experience. It was value judgements such as these that Aristotle $(1985,1995)$ spoke of in the Nicomachean Ethics when he declared the importance of the 'good' (agathos) and the 'beautiful' (kalos). For Aristotle, ethical virtue and emotion relate to the details of experience - both possess cognitive components that involve the perception of recurring relationships between particulars (Achtenberg, 2002, p. 9). The practical perception of a virtuous person occurs through the discernment of similarity and difference.

The good and the beautiful are qualities of wholeness or completeness that are brought to bear in practical perception. To perceive particulars as part of a larger scenario is to perceive their value. Hence, a virtuous person is one who ascribes meaning in details according to the wholes they may compose, such as for example,

the food before me in terms of my overall body health; the dangerous action I must pursue in terms of victory in battle, another person in terms of the joint activities we could engage in; my current activities in terms of the life goals I wish to attain; and in general, every event, situation, and thing in terms of an overall developed and flourishing life (Achtenberg, 2002, p. 9). 
Both Damasio's somatic marker hypothesis and Aristotle's ethics, suggest that our emotions are shaped by our perceptions of experience. Our emotional dispositions change according to how the situations we find ourselves in fit one or other global image of a developed and flourishing life. Hence, we come to avoid the activities, people and institutions that appear adverse or destructive to that life; and desire and seek those that complement and further it (Strauss and Quinn, 1997, p. 102-110). Our emotional responses to the situations we find ourselves in constitute our emotional responses to the world as we experience it.

Our emotionally mediated dispositions may cause us to attend to some features in our environments and ignore others. However, a dispositional state that produces one pattern within a parallel distributed network cannot be functionally separated from the dispositional state that causes another (Stich, 1996, p. 111). To isolate one disposition from another, so that one is deemed causally active in one cognitive episode and the other is not, appears to be an arbitrary act. Clark and Chalmers (2002, p. 647) argue that while some mental states, such as experience, may be internally represented, there are other types of mental activity that are dependent upon external representations in environment, culture or artefacts. By this view, beliefs are distributed, not in a shallow, insignificant way but in a deep, significant way.

The land and sea rights conferred on indigenous Australians by the Mabo decision of the High Court of Australia (1989) may be interpreted according to such distribution of beliefs. The dispute settled by the Mabo case arose because of the contrasting values placed on indigenous identity and customs within Australian society. The High Court decision acknowledges the right of indigenous Australian people to inherit land from their ancestors, so that they may maintain their identity and customs and enjoy their native title. A similar point was presented in the fictional Australian motion picture comedy, The Castle, in which the main character, Darryl Kerrigan, goes to the High Court of Australia. There, he fights an airport authority's 
attempt to evict his typical 'Aussie battler" ${ }^{, 73}$ family from their home, which is a place of cherished memories. With the help of a retired Constitutional Lawyer, he wins the case and retains the title to his home together with 'a bloody good set of gates'.

As Hutchins (1995, p. 173) explains, when we attribute to individual minds that which can only be accomplished by many individuals, who in turn are only able to accomplish what they do because of the enabling structures of cultural artefacts and norms, then we attribute to individuals something they cannot possess. Similarly, it is a mistake to forget that the powerful formal serial sentence processes that have contributed so much to human thought and accomplishment came into existence through distributed processes scaffolded by culture, environment and artefacts. To treat these as purely disembodied processes is to lose sight of their origins in a universe inhabited by humans.

\subsection{World}

Explaining cognition in causal-computational terms requires that we also understand how we conduct our business in the environmental niches we find in the world (Churchland and Sejnowski, 1992, pp. 141-238). This requires that we understand how the neurons in our brains collectively function in representing the environment. This problem becomes amenable to solution when a vector coding model of neuron activation is used in preference to a local coding model. Hence, the processes by which the environment is represented in the human brain becomes 'distributed representing' (also known as 'state space representing' and 'multidimensional representing'). The advantages of vector coding over the local coding of information include a better fit with empirical evidence, a more flexible representational model, and a stronger account of human learning capabilities. Importantly, the vector coding that occurs in a human brain only performs a portion of the computational work. The remainder of the work is represented in the cognitively significant 'contours' of the environment. These contours are formed by

\footnotetext{
${ }^{73}$ An 'Aussie battler' is symbolic of Australians who persevere in the face of the 'battles' of life. The 'Aussie battler' has been given mythological status by Australian media commentators and politicians.
} 
the features of the physical environment, the computational information (or internal contours) of artefacts such as language and physical devices, and the routines and boundaries of culture.

Language is an artefact of human social interaction that must be included in an account of our learning capabilities. The important role of language in culture arises from its ability to code information in both local and distributed ways. Language is used to store information locally in books, electronic media and diaries. Language provides a means for simplifying concepts by localising them in signs and labels and the definitions of words. Language improves our ability to learn by providing a means for chunking ideas, complementing our biological memory storage capabilities, and for guiding our thinking along the language-induced contours of our cultural environments. The structure and cadence of a poem, for example, can evoke thoughts and experiences that the words alone cannot (Clark, 1997, p. 208). Language also distributes information in those literal and non-literal phrases that refer to other concepts, times and places. These phrases draw their meaning from the connections they convey and the patterns they complete. The serial sentence character of language is complemented by its utility in conveying ambiguous meanings through propositional attitude sentences, and in conveying holistic emotionally relevant content through non-literal language forms.

Leven (1994) proposes a model for the representation of knowledge that takes into account the variety of ways language conveys emotionally and culturally relevant information. His model employs semiotics, 'a theory of the nature of signs and the structure of thought' (Leven, 1994, p. 66) and is designed to incorporate the sequential symbol processing of logical reasoning, with the insight and meaning elicited by parallel distributed processes. Various findings of neurophysiological research are cited in support of Leven's approach, including the hypothesis that neurotransmitters serve as representational media within the brain. Here, the physiochemical state of neurotransmitters is believed to vary continuously whilst also contributing to 'state-dependent memory' (Leven, 1994, p. 70). 
This state-dependent memory depends in part on the properties of networks of protein crystals. These crystals, which act as neuroreceptors in the synapses, also play a critical part in neural processing (Grant, 2003a). The synapses exist at the boundary of neighbouring neurons where information is transferred by a combination of electrical and chemical processes. These chemical processes depend on the various properties of neurotransmitters and their relationships to the macrostructure of protein crystals. When these processes result in extremely high or low states of activation, the interest and motivation of subjects is affected as well as their ability to deal with problems. With the development of increasingly sophisticated and powerful technologies, the physical nature of human cognition is becoming increasingly accessible to analysis.

Despite advances in our knowledge of the causal-computational processes of human cognition, a phenomenal dimension to human cognition remains and should be taken into account. Important to Leven's (1994) model is the role played by the social milieu as a collective 'macroparameter' that determines the way meaning is attached to raw phenomenal data. He describes the case of a Necker Cube, six lines drawn on a page to represent a transparent cube ambiguously.

\section{Figure 7.4 A Necker Cube}

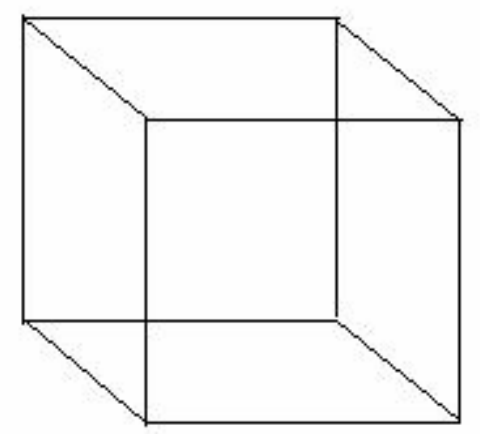

Western viewers were found to recognise one representation or another of the cube in the form of a particular perception of three-dimensional depth. However, viewers are often able to realise the other representation of the cube, when it is explained to them. One or the other representation can be represented by parallel 
distributed processes. However, prior learning experiences and expectations ensure that one of the two representations is perceived before the other. Serial sentence processing (i.e. talking to the viewer) can prompt the viewer to the possibility of another representation. When the second representation is seen, it is seen suddenly, rather than gradually, so that the entire cube is perceived at once (this phenomenon is often referred to as a 'Gestalt switch').

Interestingly, when viewers from a 'primitive' non-Western culture were presented with the cube, they were only able to perceive a jumble of lines. This response may be explained in terms of culturally mediated experiences:

To a person not trained to open cereal boxes, for whom street corners are irrelevant and tic-tac-toe squares meaningless, these apparently selfevident qualities are strange and lack explanatory power. Corners are not part of his or her experience (Leven, 1994, p. 71).

The general conclusion Leven reaches is that all learning processes are context dependent. Therefore, in the absence of a scheme for representing knowledge - a scheme that is based on contextually relevant experience - a learning response is not possible. This conclusion is consistent with Minsky and Papert's (1988) findings about perceptrons, which are machines constructed to perform parallel distributed processing. They conclude from their mathematical analysis that any learning machine, regardless of whether it is constructed to function in a serial or parallel fashion, cannot learn unless it possesses an appropriate scheme for representing knowledge.

No machine can learn to recognise $\mathrm{X}$ unless it possesses, at least potentially, some scheme for representing X (Minsky and Papert, 1988, p. xiii, italics in original).

Machines do not exhibit emotions. In humans, however, it seems that emotions do not arise primarily from serial symbol processing. Instead, emotional responses arise holistically from sub-symbolic parallel distributed processing at the level of neurotransmitters and entire systems of neural networks. Hence, Minsky and Papert include both serial and parallel systems in their model of mind: 
Most probably, we think, the human brain is, in the main, composed of large numbers of relatively small distributed systems, arranged by embryology into a complex society that is controlled in part (but only in part) by serial, symbolic systems that are added later. However, the subsymbolic systems that do most of the work from underneath must, by their very character, block all the other parts of the brain from knowing much about how they work. And this in itself could explain how people do so many things yet have such incomplete ideas of how those things are actually done (Minsky and Papert, 1988, p. 280).

According to Penrose (1989, p. 398), there is no difference in principle between a parallel and a serial computer because both are, in effect, Turing machines. A Turing machine is one in which the hardware (that is, the physical part of the machine including printed circuits, transistors, wires, magnetic storage space, and so on) has achieved a suitable degree of complication and flexibility. Such a machine is capable of simulating (at least in principle) any other machine and is therefore equivalent to any other machine. Here, the term 'equivalence' refers to the machine's ability to perform certain functions without regard to its efficiency (such as, the time taken to perform specified functions, or the memory resources used in performing those functions). Hence, the difference between parallel and serial architectures in computing devices is an efficiency difference, not a difference per se.

It seems to be the case that human cognition utilises both serial and parallel architectures. If so, then the pervasiveness of both situational and grand narratives in human thinking could simply reflect that state of affairs. Hence, we have situational narrative, with its holistically enacted emotional content and ambiguous, multireferent, propositional attitudes, standing in contrast to grand narrative, with its symbolic, serial sentence, logical content in the form of theoretical propositions.

Emotions are evident in physiological responses, culturally mediated propositional attitudes, narratively expressed propositional attitude sentences, and non-literal expressions. They play an essential role in decision-making processes involving the selection of appropriate fields of attention and the motivation of people to action. It is important to consider the role of emotions in human action, including human actions labelled as 'leadership'. This also requires the consideration of cultural symbols and literal and non-literal forms of communication. 


\subsubsection{A neural model of narrative structure}

If the formation of narrative from the novel events of experience can be explained in terms of the cognitive functions of the human brain, then such an explanation will need to be put in terms of the functioning of neural networks and other physiological systems such as the endocrine system. In the following account, an attempt has been made to give such a functional account by combining neural anatomy and physiology with a mathematical model of a parallel distributed processing system. Here, Pribham (1994) investigates processes of knowledge representation and inference by analysis of biological neural networks, as found in the nervous systems of animals and humans, and artificial neural networks. He sets out to explain the functions of the far-frontal cortex of the cerebral hemispheres of the brain $^{74}$ in terms of narrative structure.

Pribham (1994) defines narrative structure in terms of episodes, events and narrative $^{75}$. Here, he defines an episode as a unit of action that specifies a situation or context, an event comprises the particular action and its possible outcomes, and narrative is the act of making known a course of events and their consequence. Each episode is bounded by specific actions that attract a relatively high level of attention from the subject. Narrative, as conceived here, arises from the ordering of these episodes and the consequences of a course of events experienced by the subject. The recombination of episodes produces novel events, that is, new conceptions of actionand-possible-outcomes.

\footnotetext{
74 The far-frontal cortex is located in the cerebrum; the anterior (forward) and upper part of the brain, and consist of a layer of grey matter, composed of the central bodies of brain cells. Churchland and Sejnowski (1992, pp. 29-37) report that these cells are widely connected with other parts of the brain through long fibre tracts and may be associated with coherent systems (such as the visual system); though the various systems are not independent of each other and are difficult to define and separate. ${ }^{75}$ Pribham (1994, pp. 380-388) formalises these definitions by means of vector calculus.
} 
Neurophysiological evidence (Pribham, 1994, pp. 376-7) ${ }^{76}$ supports the view that the spatial and temporal boundaries of episodic experience are demarcated by the amygdala. For demarcation to take place, some process is needed to separate specific actions or context items from a continuous stream of sensory experience. Damasio (1994, p. 128-68) provides a plausible explanation for demarcation processes when he links the amygdala and other parts of the limbic system to the formation of primary emotions. Primary emotions are involved in the mental evaluation of experience and dispositions to particular experiences. Emotion serves to highlight certain events or context items from others by heightening the subject's attention to those things and separating them from an otherwise steady stream of sensory data. Emotions also play an indispensable role in rational thought:

The action of biological drives, body states, and emotions may be an indispensable foundation for rationality. The lower levels in the natural edifice of reason are the same that regulate the processing of emotions and feelings, along with global functions of the body proper such that the organism can survive (Damasio, 1994, p. 200).

In humans, the evaluative and dispositional processes that govern attention and memory occur below the level of consciousness but may also shape conscious thoughts and memories. Memories are formed from the elements of sensory experience that attract the attention of the subject. The demarcation and memory of an experience also involves the hippocampus, which is located with the amygdala in the limbic system of the brain (Carter, 1999, p. 17). Churchland and Sejnowski (1992, pp. 243-50) report the critical role of the hippocampus in the formation of memories - due partly, perhaps, to the location of the hippocampus in a region of

\footnotetext{
${ }^{76}$ Pribham (1994, p.376) reports carrying out surgical alterations, including amygdalectomy, on the brains of live monkeys. The purpose of amygdalectomy is to track the consequential effects on species-shared behaviour patterns: fighting, fleeing, feeding and sex. Since experimental lesions cannot be legally or ethically performed on human subjects, many questions regarding the human brain are approached indirectly by carrying out such surgical procedures on animals such as monkeys. Churchland and Sejnowski (1992, pp.429-50) report that most of what is known about the microorganisation of nervous systems has come from research on animal brains. Of course, the idea of surgical research on animals raises its own ethical and theoretical questions. In addition to any distress experienced by animal subjects, there are inescapable limitations to applying such research to human brain function: significant differences exist between the brains of different species and higher level cognitive functions are found only in humans. Nevertheless, some fundamental principles about brain function may be derived from animals and extended to models of the human nervous system.
} 
convergence so that it acts as a crossroad for information from the cortical areas of the brain and the brain stem ${ }^{77}$.

Pribham (1994) explains that the hippocampal systems of the brain play a part in the recombining of episodes to infer new events in memory. The ordering of these episodes in the far frontal cortex can be compared to the formation of narrative structures. Referring to models devised by a number of writers, Pribham provides several possible explanations for the practical inference of novel events: put simply, novel events are formed in memory by comparing prior experiences with the sensory inputs from current experiences. However, a novel event must cohere in some way with prior experience if it is going to make sense to the subject.

Coherence is the defining attribute of an episode, so inconsistencies or variations between patterns of activity, or covariance, must be sorted out. This sorting out process may occur through familiarity with similar types of covariance, or by classifying an episode as being equivalent or unique to other episodes. The subject must have a detailed familiarity with similar types of covariation ${ }^{78}$; or identify similarities between experiences ${ }^{79}$; or make sense of a novel experience by reconstructing it from selected elements of previous experiences ${ }^{80}$. If covariance cannot be sorted out, it may not be possible to order events, achieve coherence, and therefore make sense of the new experience.

As has been explained previously, sense is made of new experiences by drawing on prior experiences. Information processing that utilises a familiar episode, as a

\footnotetext{
77 The brain stem plays an important role in maintaining alertness and attention (Carter, 1999, p.183).

${ }^{78}$ In this case, the subject makes sense of a new experience by identifying similar patterns of variation between other experiences. For example, I may identify the set of equivalent elements and the set of different elements existing between two ways of paying my electricity bill (paying it in person with cash verses paying by electronic transfer on the internet). If so, it becomes easier for me to make sense of the similarities and differences between the familiar experiences of buying a book in a shop at the local shopping centre and, say, the new experience of ordering a book electronically on the internet. ${ }^{79}$ For example, hailing a taxi in Melbourne will share some similarities with the process of hailing a cab in New York.

${ }^{80}$ For example, whilst on a visit to New York, I may hail a cab to go to an internet café in order to order a book on the internet. In doing so, I may sort out this experience by drawing on the relevant elements of previous separate experiences such as hailing a taxi in Melbourne, getting internet access in my local library, and buying a book in a bookshop.
} 
reference for assigning value to unfamiliar episodes, involves the amygdala. Different types of episode evoke different neural responses and therefore different responses in the subject. When sensory input does not lead to the complete specification of an event and its domain, the systems of the frontal lobe may become involved. Coherent properties become assigned to an event by inference based on prior experience. Such experience is more than just remembering the past; it is also involves the creation of a coherent future from a set of plausible outcomes; that is, it involves the creation of narrative.

The neural model of narrative structure presented here assumes a plasticity of cognitive machinery that enables the creation and modification of memories. This capacity is important to learning. Since learning involves many different elements of representation working in concert, it is a special case of distributed cognition.

\subsubsection{Learning}

Learning is a process that involves mind and body and world. When a child begins to walk, a teenager learns to drive a motor car, a mother learns to care for a baby, or a novice principal learns to manage a school, learning is evident in the changed behaviour of those individuals. To an external observer, the outworking of those changes appears orderly and orchestrated because they are due to changes in the cognitive patterns within the person. These patterns are the outcome of a process in which the physical structures of a parallel distributed processing system, and the information it processes, conjointly exhibit stability and adaptability. Both of these characteristics may be subsumed within the term 'plasticity', which lies somewhere between the fixed order of unchangeable rigidity and the disorder of an amorphous fluid. Churchland and Sejnowski (1992, p. 239) attribute the ability of the brain to mediate learning to the continuous modification and updating of information; and this occurs due to the collective plasticity of neural cells:

Brains are continuously modifying and updating themselves. Virtually all of a brain's functions, including perception, motor control, thermoregulation, and reasoning, are modifiable by experience (Churchland and Sejnowski, 1992, p. 239). 
The representations distributed across the individual cells or units of a parallel distributed network generate its outputs. This applies when there is any significant degree of complexity in those representations.

Learning within an individual is instantiated as a global property arising from local changes in cells. As Churchland and Sejnowski (1992, p. 241) explain, knowing how an individual unit changes in response to input from other units will not in itself divulge the cell's contribution to learning in the network. Neither will it provide helpful information about the pattern of changes in the population of units nor the principles that bring about the formation or dissolution of those patterns. The mechanisms of plasticity that enable some characteristics of neural patterns to be conserved whist others are modified need to be explained in terms of collective changes to populations of neurons. According to Churchland and Sejnowski (1992, p. 250), it is possible for neuron connectivity to be changed in response to a number of events, including:

- the extension of dendrite branches or the sprouting of new dendrites;

- changes to existing synapses or the creation of new synapses;

- the number of dendrites being decreased or parts of dendrites removed so as to decrease the number of synapses;

- the shutting down of existing synapses;

- various micro changes that result in changes to axons or presynaptic processes; or

- the death of the cell itself, taking with it all the synapses it formally supported.

Although this list of possibilities includes several of the structures and mechanisms associated with neurons, they all result in changes to the number and strength of synaptic connections between neurons. Whilst knowledge of the plasticity of neurons is important to an understanding of human cognition, it is far from sufficient. Attempts to develop autonomous robots have led to a rejection of the classical image of the mind in favour of a decentralised, embodied model of 
cognition. The classical view portrays mind as a purposeful, central executive, or planner, with access to all the information anywhere within the system, and with the ability to control all the actions of the system. Adherence to this classical view also supports a classical view of leadership, whereby the leader is the central executive who plans and coordinates action.

\subsubsection{The impracticality of a central planner}

As Clark (1997, p. 21) says, 'the trouble with a central planner is that it is profoundly impractical'. Coordinating decisions through a single central location requires that sensory information be converted into symbolic code for transmission to the central executive and the corresponding transmission of code from the central executive to other parts of the system. This creates an information bottleneck that severely hinders rapid real-time responses. A model that distributes information throughout a system, so that multiple sub-systems can work in parallel, enables numerous sources of information to be processed simultaneously. If the system is strongly interconnected, it will maintain the coherence required for unified action, thus removing the need for a central executive to coordinate the actions of the subsystem.

Central executive models of organisational functioning are unable to account for the many decisions made in organisations every day. Undoubtedly, some decisions are more visible than others are, but highly visible macro-decisions originate from the many smaller, less visible decisions and actions that precede them. For example, a decision to replace a school's existing buildings with a new multi-million dollar complex is not likely to be made in isolation from prior decisions. If an informed and rationally justified decision is made, those who fund the building program, the policies and regulations that pertain to school building works, education policies, and the views of the relevant principal and school community will be taken into consideration $^{81}$. The implications of a parallel distributed view of organisations are that numerous decisions and actions are taken by many individuals and groups of

\footnotetext{
${ }^{81}$ See for example, Parkdale Secondary College Gets Green Light for New Buildings (2006).
} 
people, and those decisions and actions influence future decisions and actions. Bottlenecks are removed through the internal and external distribution of organisational functions so that multiple decisions and actions can be taken simultaneously. Importantly, the distribution of functions occurs by default even though the communications that facilitate the distribution of information may take the form of serial sentence processes. A command and control hierarchy may be put in place in an organisational structure, but that structure will continue to depend on parallel distributed processes if it is to function at all. Indeed, a school principal who acknowledges this reality will take structural and procedural steps to facilitate the flow of information between organisationally relevant units (students, parents, teachers, classes, faculties, etc.) and decentralised decision-making processes.

Another consequence of parallel distributed processing is that it creates the possibility space for an advantageous sensitivity to environmental features, labelled in 1934 by Jakob Von Uexkull (Clark, 1997, p. 24-25) as 'Umwelt'. For example, the Umwelt of a tick is constituted by the tick's sensitivity to butyric acid, a cue that enables it to detect the presence of nearby animals from which it can draw blood. Hence, the tick's 'effective environment' is defined by its particular lifestyle, which is focused on its need to reproduce and therefore its need for blood. The phenomenal perception of humans might also constitute a type of Umwelt by which individuals become conditioned to noticing particular cues or features in their environment. Clark illustrates the contrast between a formally theoretical and phenomenological account of perception by reference to the act of catching a ball:

Consider the act of running to catch a ball. This is a skill, which cricketers and baseball players routinely exhibit. How is it done? Common experience suggests that we see the ball in motion, anticipate its continuing trajectory, and run so as to be in a position to intercept it (Clark, 1997, p. 27)

Quoting videotaped evidence, Clark cites empirical evidence supporting the view that Umwelt is at work in the sporting players:

A more computationally efficient strategy is to simply run so that the acceleration of the tangent of elevation of gaze from fielder to ball is kept 
at zero. Do this and you will intercept the ball before it hits the ground (Clark, 1997, pp. 28-29).

Hence, it is the phenomenal experience of the catcher that is centrally important, rather than an executive driven theoretical perspective. This is also true for leadership, as those in formal leadership positions perceive their roles in ways that make sense to themselves. For example, Australian Cricket Captain from 1999 to 2004, Steve Waugh, maintained his resolve with self-talk. He reports saying to himself, 'Follow your instincts as a captain. Don't do it by consensus - lead by example' (Waugh, 2005, p. 549). This statement reflected the circumstances he was facing at the time and his reflections on his captaincy. At the time, the team perceived a lack of decisiveness in Waugh, so he took steps to change their perceptions of his leadership ability by changing his perception of himself. Whilst recognising that he had an important job to do, which needed to be done well, he also acknowledged he had no control over many important factors. For example, he found himself a powerless and awestruck spectator when his brother, Mark, drew on his own experience to take a remarkable catch:

As far as sporting wonders go, the catch taken by Mark Waugh in the second Test, in Hobart, to haul in a late cut from Inzamam-ul-Haq must be right up there with the most freakish of all. That the catch turned the tide of a match that was drifting hopelessly out of our reach only added to its greatness. It was an effort that needed remarkable anticipation backed up by incredible hand-eye coordination (Waugh, 2005, p. 554).

A great deal of human intelligence utilises heuristic context-specific tricks and strategies. It is strategies of this sort that scaffold problem-solving, discovery and creativity to mould and orchestrate behaviour in favour of global coherence. Hence,

The Rational Deliberator turns out to be a well camouflaged Adaptive Responder. Brain, body, world and artefact are discovered locked together in the most complex of conspiracies. And mind and action are revealed in an intimate embrace (Clark, 1997, p. 33).

Artefacts, culture and environment scaffold the physiological patterns produced in the body by neurons and hormones. Artefacts are the product of human wisdom, obtained through many repetitions of adaptive cognitive work, sometimes over 
centuries. Cultural norms constitute the routines obtained because of the collective cognitive work of many people, and obtained in the interest of survivability, productivity and harmony. The environment too, provides potential scaffolds and impediments to human action, which we have learnt to exploit or negotiate in countless ways - countless, because each variation of environment requires its own special solutions. As a new environment is encountered, well rehearsed strategies that have borne fruit in environments with similar features can be called upon. Sometimes those strategies can be implemented without significant change; sometimes they need to be adapted to the features of the new environment; and sometimes an existing repertoire of strategies needs to be supplemented with a completely new strategy. Hence, Clark (1997, pp. 103-128) argues that a successful cognitive science will be one that studies the larger dynamics of agent-environment systems as well as the computations of neural circuitry.

\subsubsection{Levels of analysis}

An analysis of a parallel distributed processing system can be broken down into multiple levels of analysis. The macro-meso-micro triad, which was introduced in Chapter 3, can be used for this purpose. When an analysis is of the work of schools is conducted, the levels of analysis may be structured as shown in Table 7.2.

Table 7.2 Distributed cognition: levels of analysis

\begin{tabular}{|c|c|c|c|c|c|}
\hline $\begin{array}{l}\text { Focus of } \\
\text { analysis: }\end{array}$ & $\begin{array}{l}\text { Educational } \\
\text { Culture }\end{array}$ & $\begin{array}{l}\text { A school } \\
\text { system }\end{array}$ & A school & $\begin{array}{l}\text { A school } \\
\text { faculty }\end{array}$ & $\begin{array}{l}\text { Student } \\
\text { learning }\end{array}$ \\
\hline $\begin{array}{l}\text { Macro } \\
\text { Level: }\end{array}$ & Environment & $\begin{array}{l}\text { Societal } \\
\text { Culture }\end{array}$ & $\begin{array}{l}\text { School } \\
\text { system }\end{array}$ & $\begin{array}{l}\text { Academic } \\
\text { discipline }\end{array}$ & Curriculum \\
\hline $\begin{array}{l}\text { Meso } \\
\text { Level: }\end{array}$ & $\begin{array}{l}\text { Societal } \\
\text { Culture }\end{array}$ & $\begin{array}{l}\text { School } \\
\text { System }\end{array}$ & $\begin{array}{l}\text { School } \\
\text { Culture }\end{array}$ & $\begin{array}{l}\text { Faculty } \\
\text { Culture }\end{array}$ & $\begin{array}{l}\text { Student } \\
\text { Culture }\end{array}$ \\
\hline $\begin{array}{l}\text { Micro } \\
\text { Level: }\end{array}$ & $\begin{array}{l}\text { School } \\
\text { System }\end{array}$ & School & $\begin{array}{l}\text { Sub-school } \\
\text { or Faculty }\end{array}$ & Teachers & Student \\
\hline
\end{tabular}


An educational culture is affected by the physical environment in which it functions. For example, a school system that overseas education in the environment of the vast rural regions of central Australia will face challenges that are not experienced to the same degree by a school system servicing the towns and cities of the south-eastern region of Australia. Similarly, a societal culture that mainly comprises people of indigenous and Anglo-Celtic descent will possess educational needs and challenges that differ in particular ways from a societal culture influenced by a multicultural mix of people of Asian, Anglo-Celtic, European, and Pacific descent. Each situation will present its own environmental, cultural and artefactual particulars, and should be analysed in accordance with the effects presented by those particulars.

When an analysis is focused on a school, the physical environment will include the region in which the school is located, but also the internal physical environment such as the characteristics of learning and working spaces. A traditional school design typically organises classrooms into segregated spaces linked to a common corridor, and containing tables organised in rows to face a writing space such as a blackboard or its modern electronic equivalent. This type of arrangement is optimised for individual teacher instruction and teacher directed learning activities. However, if an open plan classroom design is adopted, with flexible access to a variety of workspaces, different types of educational experiences become possible, whilst others become more difficult to implement.

The information relevant to an analysis will be distributed, almost certainly, across the physical environment of a school, the prevailing culture in which it functions, and the artefacts in use. Similarly, if the unit of analysis is an individual person the relevant information will be distributed across her mind and body, the spaces in which she works the tools and technological devices she uses, and the cultural norms and characteristics of the people she works with. An investigation of this sort might take place in relation to a performance and development review, or a research case study of the practices of an individual teacher. This kind of case study would differ in significant ways from a narrative analysis (such as that conducted in this study) because its focus would be on causal-computational processes. 
Much remains hidden in the analysis of schools, because the collection of the relevant data is not feasible, or is not considered relevant. Yet, in some circumstances, that information may of great importance. For example, a naturalistic study of the physiology of student learning experiences would require information that is difficult to obtain, but which would enable student's cognitive processes and emotional experiences to be examined. The body has information distributed across the nervous, endocrine and other physiological systems, with the nervous and endocrine systems sharing the hypothalamus. Amongst the nervous and endocrine systems, information is distributed across neural networks, glands and organs. Within the nervous system, information is distributed across neurons connected in parallel; whilst the endocrine system distributes information via chemical processes. Within the synaptic connections between neurons, information is distributed across networks of protein crystals. Each of these processes may provide significant information if the data about those processes can be obtained and analysed in accordance with distributed cognition theory.

\section{Figure 7.5 Parallel distributed processing - Levels of analysis}

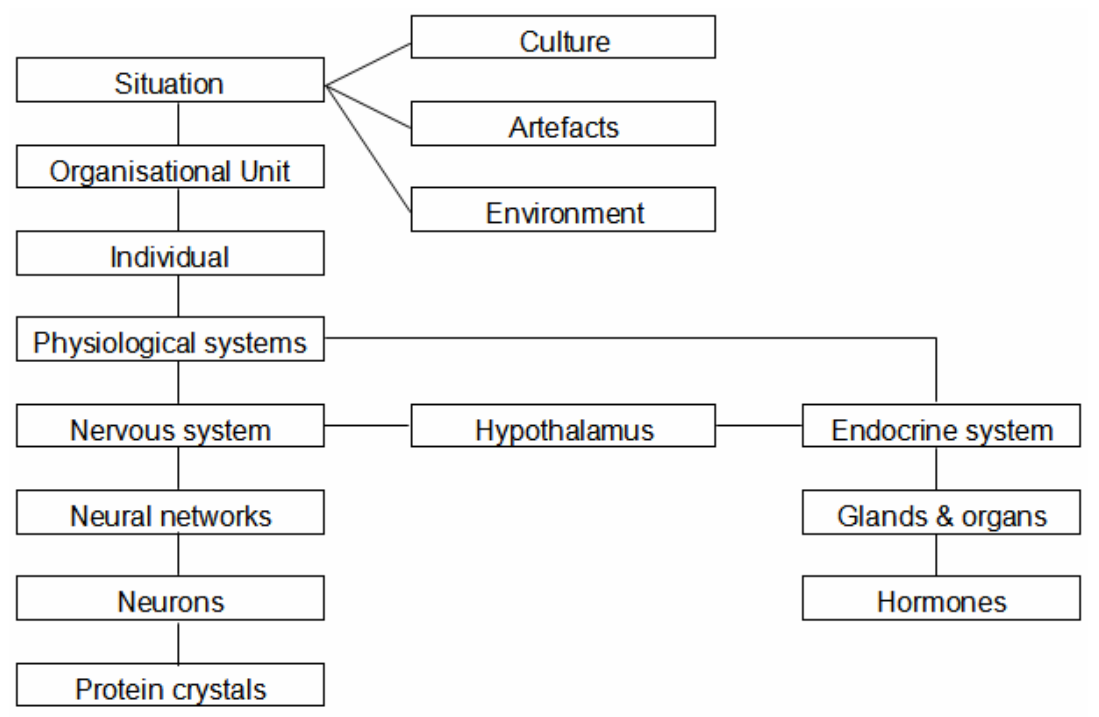

A single situation contains a vast array of information-sharing units, predominantly connected in parallel. Any one unit represents a tiny portion of the total array of information present in a situation. The composition of that information 
will be influenced by the number, strength and distance of connections between a single unit and other units. In graph theory, as reported by Grant (2003b, p. 1232), networks are represented by linked nodes. As shown in Figure 7.6, the simplest networks, known as random networks are those composed of randomly linked nodes, such that each node has a similar number of links. A network with occasional longrange connections between nodes and a small number of highly connected hub nodes gives rise to a heterogeneous network known as a scale-free network. Scale-free networks possess a 'small world' property, which is explained in terms of the shorter, more direct connections between individuals.

\section{Figure 7.6 Random and Scale-free network configurations ${ }^{82}$}

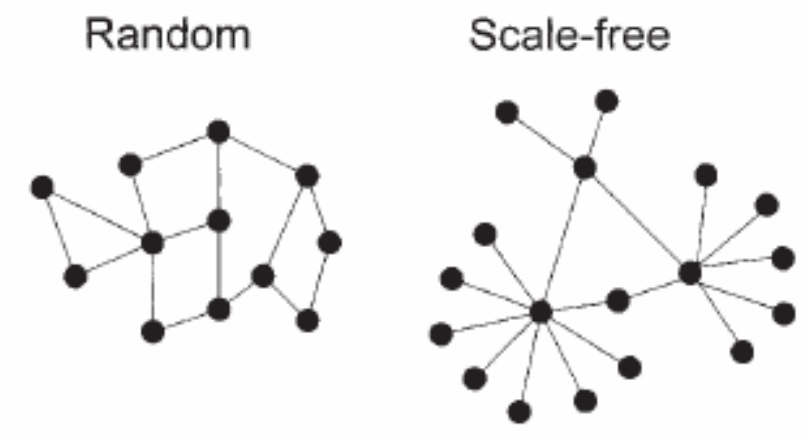

Similarly, the causal-computational interactions occurring in a school may be analysed according to the number of connections occurring between the units, or nodes, of analysis (such as schools in a school system, faculties or sub-schools in a school, teachers within a learning unit, and so on). What such an analysis might reveal is the type and frequency of information transfer and the manner in which that information is processed by each node before being passed on.

\subsection{The distributed mind}

To the extent that human cognition comprises distributed computational processes, the activities of humans must arise from those processes. Hence, if

\footnotetext{
${ }^{82}$ Source: Grant, 2003b, p.1233, Fig. 2.
} 
particular kinds of human dispositions or activities can be described as leadership, those dispositions and activities will depend, at least in part, upon distributed cognition. Consequently, if it were possible to describe leadership in computational terms, then it would be appropriate to describe it as distributed leadership.

Distributed cognition theory is a theory that can be evaluated according to a naturalistic coherentist epistemology. It can explain organisational functioning in terms of the dynamic interaction of mind, body, and world, where world comprises artefacts, culture and environment. Leadership, however, is not amenable to such an analysis. Formal leadership theories have not succeeded in providing a systematic account of leadership as a concept with causal properties. Perceptions of leadership vary in the same way that perceptions of colour vary. The one colour, naturalistically defined, may be perceived differently in darker or brighter light conditions or against backgrounds that vary in colour, brightness or density; or perceived differently by different observers with different background experiences, or from different standpoints. Leadership takes on the character of a phenomenal quality, which obtains its meaning through the perception of difference obtained from subjective feels, or qualia.

In the following chapter, I put a case for phenomenal accounts of leadership being placed alongside naturalistic accounts of organisational functioning, within a theory of distributed leadership. This theory is a special case of distributed agency, which allows the purposeful action of human agency to stand alongside a causal account of organisational functioning. Hence, a naturalistic account of organisational functioning is placed alongside a phenomenological account of leadership. 


\title{
Chapter 8: Distributed Leadership
}

\begin{abstract}
As argued in the previous chapter, distributed cognition is compatible with a naturalistic coherentist epistemology and can explain organisational functioning in terms of the causal-computational interaction of people, culture, artefacts, and environment. Whilst this account coheres with accepted scientific accounts of human cognition from a third-person perspective, the phenomenological accounts of leadership speak of the 'raw feels' of human intentionality from a first-person perspective. A theory of distributed leadership acknowledges that these two perspectives of human organisational life share the same information space. Distributed leadership in schools is a theory that places a formal, naturalistic account of organisational functioning alongside an informal, phenomenological account of leadership. This two-dimensional account of organisational life takes both an 'outside-looking-in' and 'inside-looking-out' perspective of organisational life.
\end{abstract}

\subsection{Introduction}

"Mine is a long and a sad tale!" said the Mouse, turning to Alice, and sighing. "It is a long tail, certainly," said Alice, looking down with wonder at the Mouse's tail; "but why do you call it sad?" (Carroll, 1984, p. 36).

Lewis Carroll's pun relies on the differentiation of intangible qualities and physical objects. Similarly, the intentionality of narrative contrasts with the causalcomputational accounts of naturalism. Whilst the causal-computational processes of distributed cognition provide a fruitful means to the study of organisational functioning, it does not capture the ambiguity of organisational life as perceived by those who experience it. Ambiguity appears also in the abstract content of leadership narratives, with their reliance on subjective, first-person perceptions of organisational life. Whilst these phenomenological perceptions rely on propositional attitude sentences and the 'raw feels' conveyed by non-literal language, the theoretical propositions of science, describe organisational events in terms of concrete reality and explicitly defined events. In his narrative history of the Great War, Carlyon (2006) contrasts the high casualty rate of the Allied armies, early in the war, with the more systematic strategies implemented by generals towards its end. Early generals, 
he argues, built their strategy upon phenomenal qualities such as courage, moral purpose and personal loyalties. Later generals, however, learnt from the mistakes made early in the war and conceptualised military strategy in terms of science, engineering and logistics:

Before the Great War was over it would throw up 'modern' men, notably the Canadian Arthur Currie and the Australian John Monash. But in 1916, and even though it had seen what machine guns and howitzers could do to men advancing in extended lines, the British army was still running on Victorian and Edwardian values. Among these were courage and high moral purpose, and also cronyism, rivalries that sometimes had a feline edge, and patronage (Carlyon, 2006, p. 44).

A similar distinction between phenomenological qualities and naturalistic, cognitive processes may be found in other organisational contexts, including schools. According to Margaret, a leading teacher, teachers in her school had more of a sense of shared purpose than they did in the previous year:

I think Sarah [the Principal] worked very hard last year to get people's confidence in herself and how she operates as a team. I think it all reflects back on Sarah because I think she is a lot more approachable to everybody this year and people feel that they can speak to her. I don't know, maybe it's a combination of the people that we have in the school this year (Interview B8).

At first, Margaret explains this change in terms of the phenomenological qualities of leadership, as expressed in the phrases, 'to get people's confidence', 'being approachable', and 'people feel they can speak to her'. Then, as she appears to reflect on her words, Margaret changes tack and begins to speak of the composition of the teaching staff, of teachers deepening and sharing their knowledge, and supporting one another. As she does so, her analysis shifts from leadership related phenomenological qualities, to a description that fits within the naturalistic account of distributed cognition theory with its emphasis on culture and learning.

In Chapter 6, I argued that leadership is a phenomenological concept of folk psychology, whereas in Chapter 7, I put a case for explaining organisational functioning according to the naturalistic, causal-computational processes of 
distributed cognition theory. In this chapter, I discuss the feasibility of a theory of distributed leadership, which describes organisational functioning in terms of the causal-computational processes of distributed cognition theory, whilst also acknowledging the resilience of phenomenal perceptions of leadership in folk psychology. Whilst the predictive, explanatory power of distributed leadership lies in the causal and computational terms of distributed cognition theory, it also acknowledges the perceived values and qualities, or 'raw feels', that make up the reality of those who participate in organisational life.

In acknowledging the phenomenally perceived concepts of folk psychology, I have adopted a theory of mind that acknowledges the capacity for humans to make free-will choices. Free-will and intentionality persist as concepts of folk psychology. Consequently, much of everyday, commonsense reasoning, including the everyday reasoning of people working in schools, assumes that people have freedom (in a cognitive sense) to focus their attention on particular aspects of organisational life and to choose between competing options. This folk psychological reasoning is the sort of reasoning that practitioners typically use in their day-to-day dealings with people in organisations.

Whilst an important part of a researcher's role is to contribute to the theoretical analyses made by those in the research community, another significant aspect of a researcher's role is to communicate findings to practitioners in comprehensible ways, and consequently, to indirectly influence day-to-day practice. Influencing those who work in schools, therefore, requires that research acknowledge principals' and teachers' intuitions about their work and the consequences those intuitions may have for their practice. That is, practitioners need to be able to relate research to what they already know, as represented in their core theories about the world, so that they may learn from research findings.

The concept of leadership assumes intentionality, such that leaders and followers are able to choose between alternative actions or goals. The assumption that people make such choices pervades everyday sense-making and underlies our justice, social and political systems - especially democratic systems. However, we are not yet able 
to explain, naturalistically, how it is that people have the freedom to choose particular courses of action. Neither are we able to explain the extent to which such freedom applies.

Naturalism differs from folk psychology because its commitment to deterministic and probabilistic causal processes does not readily admit human intentionality. The determinism of folk psychology, however, provides a bridge to understanding the principles of naturalism, which has its historical roots in folk psychology and has improved our knowledge of deterministic causal processes. Nevertheless, it may also be argued that commitment to intentionality, which is shared by both folk psychology and phenomenology, also arose as phenomenology grew out of folk psychology. Nevertheless, in folk-psychology, the free-will agency of humans constitutes a yet unexplained phenomenology of agency (Bayne, 2006).

Science offers potentially fruitful directions of inquiry for explaining the physiological mechanisms of human decision-making in terms of relations of cause and effect. However, the formal deterministic processes of naturalism must be either inconsistent or incomplete. Since naturalistic coherentism cannot admit inconsistency, the naturalistic knowledge that arises from this epistemology must therefore be incomplete. This leaves room for a phenomenology of experience to describe informally that, which cannot be explained formally by naturalism alone. The two accounts share the one information space of human experience, so may stand side by side as two perspectival descriptions of the one entity, one being objective the other subjective. The discipline of phenomenology is not as mature and reliable as naturalism, but its demise is not certain. A broad construal of folk psychology exhibits commitments to both 'agent-causation and a deterministic story about psychological processes' (Nichols, 2004) and is therefore incoherent.

The availability and scope of free-will choices may be constrained by caused events or probabilities, but the act of making a free choice, of itself, cannot be determined. Kane's (1999) view is that free-will choices cannot be caused by prior events, and that the justification of a free-will decision lies in the future, not in the past. A particular course of action may be chosen for prior reasons, which are neither 
conclusive nor decisive. Hence, decisions are not determined by prior events, nor are they the outcome of probabilities determined by prior events. Free-will decisions are taken for reasons that are not fully comprehended until after they have taken place, so that

agents who exercise free will are both authors of, and characters in, their own stories at once. By virtue of "self forming" judgments of the will (arbitria voluntatis), they are "arbiters" of their lives, taking responsibility for "making themselves" out of past that, if they are truly free, does not limit their future pathways to one (Kane, 1999, p. 240).

According to Van Inwagen (2000, p. 1), although 'a strong and unanswered prima facie case' exists for its impossibility, free-will undeniably exists and is therefore mysterious. Much of the debate about free-will hinges on its incompatibility with determinism. However, the reach of determinism does not extend to cases of strong emergence, whereby

\begin{abstract}
a high-level phenomenon is strongly emergent with respect to a low-level domain when the high-level phenomenon arises from the low-level domain, but truths concerning that phenomenon are not deducible even in principle from truths in the low-level domain (Chalmers, 2006, p. 244).
\end{abstract}

This amounts to determinism's inability to refute conscious experience, as experienced from within consciousness, so that human cognition may have both causal and phenomenal character.

Accepting the abstract concepts of phenomenology is consistent with the view attributed to Gödel, such that a deeper knowledge of the abstract concepts underlying material systems is achieved by 'cultivating intuition of essences' (Tieszen, 1998, p. 195). The phenomenology of intentionality and the causal-computational processes of naturalism need not be mutually exclusive. O'Connor (2000), for example, employs a form of property dualism to argue that free-will may be realised through the emergence of purposeful action; and Balaguer (2004) blurs the distinction between causal and non-causal events to argue that a plausibly coherent and naturalistic formulation of free-will is possible. That is, that human cognition 
exhibits intentionality. This means that cognition is always about something. Consciousness is always consciousness of [italics in original]. One can see this clearly in acts of believing, knowing, willing, desiring, remembering, hoping, imagining, and so on (Tieszen, 1998, p. 182).

Since humans cannot experience everything all at once, our attention is limited to those categories of objects or concepts that relate to our needs, interests and intentions.

The attention people give to particular kinds of concertive, purposeful action in organisations may be conceptualised as leadership. In the previous chapters, I have argued that leadership, as it is described in folk psychology, is more like a concept of phenomenology than a natural object. If leadership is understood as a case of strong emergence, as a dispersed property of natural systems, then it is compatible with distributed cognition theory. However, this gives rise, not to leadership as a property of an individual, but as a dispersed, high level property arising from low-level causal interactions. In this case, leadership is better described as distributed leadership to distinguish it from the notion of leadership as the property of a single individual.

Two consequences flow from this analysis. Firstly, an explanatory gap arises if strong emergence, which cannot be predicted from low level causal events, gives rise to the phenomenal perception of leadership qualities. Secondly, the act of distinguishing leadership properties from non-leadership properties cannot follow from a naturalistic analysis; it can only follow from a phenomenological analysis. This gives rise to a plurality of first-person interpretations of meaning and the problem of adjudicating one person's experience against the experience of others. If the adjudication occurs by means of 'democratic' processes then the problem of induction occurs. The best that can be done is a kind of coherence justification, through which the inter-subjectivity of experience is evaluated. However, if one firstperson perspective possesses core theories that differ from those of another firstperson perspective, achieving intersubjective coherence becomes problematic.

Whilst two people necessarily share a common nature, they may not necessarily share a common phenomenology of experience. An individual with adequate 
leadership qualities, as perceived by one person, may be perceived by another to be lacking in leadership qualities. That is, the intentionality of one person will not necessarily coincide with the intentionality of another, simply because that person may attend to things in a particular situation that the other may not. In organisations, however, a common naturalistic, causal-computational view is important. For example, if two scientists are working within the one space agency, with one assuming that $\mathrm{E}=\mathrm{mc}^{2}$ and the other assuming that $\mathrm{E}=\mathrm{mc}^{3}$, significant problems can be reasonably expected in the operation of the agency's satellites. Similarly, if a school principal is allocated funds to employ fifty teachers, but instead employs seventy without any further source of funding being available, then the organisational functioning of his or her school is likely to be adversely affected. Whereas a common, phenomenology of leadership is unlikely to occur, a common, naturalistic explanation of organisational functioning is both possible and advantageous.

\subsection{Leadership in organisations}

In Chapter 1, the problems of leadership were discussed. Popular views of leadership are portrayed in a matter-of-fact way in folk psychology as if the identity and properties of leadership were self-evident. Yet, the ambiguity of leadership becomes apparent when it is formalised in organisational structures. It may be said, for example, that a formal positional leader lacks the qualities of leadership or that a person who occupies a low position in an organisational hierarchy exhibits leadership qualities.

Established institutions place a high degree of cultural significance on the roles and functions of formal positional leaders and the places they take in hierarchical authority structures. Even in 'flat' organisational structures, or those that employ democratic decision-making processes, formal leaders feature within established patterns of organisational functioning. These characteristics are also evident in schools. 
Schools hold established places in societal structures and act as pathways to other important institutions. They are concerned with the production and reproduction of economic, political and cultural ways of life, including culturally fashioned notions of what leadership is, or what it should be. Schools are charged with 'raising up leaders' and school administrators are expected to 'show leadership'. However, attempts to construct formal theories of leadership have yielded contradictory and inconclusive results; partly because it is not necessarily clear what leadership is, and partly because of the epistemological problems that follow from reliance on the empirical foundations of logical empiricism or the subjective foundations of interpretivism (Evers and Lakomski, 1991, pp. 76-97).

\subsubsection{Naturalistic coherentism}

Organisation theories also have a troubled history. The application of Taylor's 'scientific management' to schools (Callahan, 1962), for example, suffers from the problem of a foundationalist epistemology and the sharp fact-value distinction of logical empiricism. The application of 'efficiencies' in education, according to this theory, simply led to the undervaluing of sound educational practices and the under funding of schools. The problems of 'scientific management' continued with the advent of social systems theories.

Social systems theories such as those put forward by theorists such as Parsons (1970), von Bertalanffy (1971), Katz and Kahn (1966), and Hoy and Miskel (1987) sought to explain organisations as systems of interactive social processes. However, the systems view is framed in terms of context- and value-free theoretical propositions. Those propositions lose their force in the absence of the fine-grained detail that is needed to explain what the system constitutes and the reasons why it functions in the way it does.

In contrast to the logical positivist approach of systems theory, the subjectivist theory proposed by Greenfield $(1975,1986)$ merely replaces the foundations of logical empiricism with foundations comprising the subjective interpretations of people. In rejecting any possibility of a scientific analysis of organisations, he fails to 
acknowledge the physical environment in which organisations function, including the mediating function of artefacts (Evers and Lakomski, 1991, pp. 76-97). He also removes any means for adjudicating between the subjective interpretations of individual subjects and the intersubjective nature of social practice.

The critical theory inspired by the social theory of Habermas (1984), does acknowledge the intersubjective nature of social practice. In doing so, it attempts to evaluate organisations in terms of the values placed on particular forms of social and political life (Rizvi, 1991). However, the overtly neo-Marxist political character of critical theory raises one set of political values over others, so that it takes on the appearance of dogma. It also fails to acknowledge the material impact of the physical environment and artefacts on human activity and organisational functioning; and tends to limit the analysis of culture to the analysis and transformation of hegemonic power relations.

A naturalistic coherentist epistemology is employed in this study. Insofar as human knowledge can be supported by evidence, this entails that epistemology itself be naturalised so that it coheres with scientific accounts of learning (Lakomski, 2005, p. 139). This epistemology draws on trusted theories of the natural sciences to explain human learning and its impact on organisational functioning. Hence, natural science and social science are drawn together into a single coherent account of organisational functioning.

Of course, all scientific knowledge is provisional knowledge and is subject to modification or replacement. Its trustworthiness, as demonstrated by the historical improvement and replacement of scientific theories, pertains to it being retained only as long as it continues to cohere with evidence and retain its explanatory power. For example, the theories of phlogiston and caloric were replaced by the kinetic theory of heat, the theory of the ether was replaced by the theory of the vacuum, and Newton's laws of motion were superseded by the General Theory of Relativity. Whilst scientific knowledge has an inescapably socio-cultural character (Kuhn, 1970; Feyerabend, 1976), it is also pragmatically useful, logically and mathematically rigorous, and amenable to both empirical and rational analysis. Unlike logical 
empiricist conceptions of science, naturalistic coherentism acknowledges the influence of culture on knowledge and allows scientific and social knowledge to share a single epistemological fabric.

Naturalistic coherentism blurs distinctions between fact and value, observation and theory, tacit and explicit knowledge, and between the knower and that which is known. Consequently, the methodology of an investigation obtains epistemological equivalence to the knowledge it realises. Since all observation is theory laden and all theory is informed by experience, the dichotomous distinctions between fact and value, or theory and observation fall away. The superempirical criteria of conservatism, simplicity, consistency, comprehensiveness, fecundity, explanatory unity, refutability, and learnability enable competing theories to be evaluated holistically, together with their subsidiary empirical claims.

\subsubsection{Methodology}

In adjudicating theories according to superempirical criteria, heuristic analysis suggests that theories of mind are centrally important to the explanation of organisational action and leadership. Accepting the existence of consciousness and free-will in a causal-computational world requires that a dualistic theory of mind be adopted. On this view, organisations constitute a single information space of human experience, which may be described in both naturalistic and phenomenological terms.

The methodology of this study includes the hermeneutic analysis of leadership as portrayed in the situational narratives of folk psychology. The schemas of situational narratives are distinguished from grand narrative by their reliance on propositional attitude sentences, non-literal language, imagery, and symbolism. The grand narratives that these situational narratives support are identified and their compatibility with formally structured leadership theories is judged heuristically. The theoretical analysis of leadership theories reveals that leadership is not amenable to global generalisations in the guise of formal, rule-governed explanations, including the causal-computational explanations of distributed cognition theory. 
This investigation utilises theoretical, hermeneutic and heuristic methods of investigation, with coherence being sought by philosophical and methodological triangulation. The three worlds of inquiry of Popper and Eccles (1983, pp. 3-50) form an organising framework for this triangulation. According to this framework, the interaction of the objective world and theoretical world is mediated by the subjective world. Whilst the nature of organisations may be perceived by virtue of their buildings, artefacts and personnel, if leadership has an objective nature, it is not clear what that nature is.

\subsubsection{Narratives of leadership}

According to Arrowsmith (2004, p.31), the analysis of distributed leadership conducted by The Hay Group Education (2004), casts distributed leadership as an interpretive concept that depends upon the characteristics of the situation, rather than a normative concept that generalises to all situations. Interpretive concepts are described in phenomenal terms.

Leadership accounts employ phenomenal terms when they attribute purposeful action to the conscious intentionality of the leader (Levinas, 1973). In the following example, concerning the events that gave rise to the 1812 invasion of Russia, Tolstoy (1991) downplays the causal efficacy of leadership whilst describing events, both in phenomenal terms and in terms of cause and effect. Here, I have highlighted, in bold type and the colour blue, those phrases that may be interpreted as being causalcomputational in character. Those phrases that constitute propositional attitudes, and therefore the phenomenal categories of intention, are highlighted in italics and the colour yellow.

The people of the west moved eastwards to slay their fellow men, and by the law of coincidence thousands of minute causes fitted in and coordinated to produce that movement and war; reproaches for the non-observance of the Continental System, the Duke of Oldenburg's wrongs, the movement of troops into Prussia (undertaken, as it seemed to Napoleon, only for the purpose of securing an armed peace), the French Emperor's love and habit of war coinciding with his people's inclinations, allurement by the grandeur of the preparations, and the expenditure on those preparations and the need of obtaining advantages to compensate for that expenditure, the intoxicating 
honours he received in Dresden, the diplomatic negotiations which, in the opinion of contemporaries, were carried on with a sincere desire to attain peace, but which only wounded the self love of both sides, and millions and millions of other causes that adapted themselves to the event that was happening or coincided with it (Tolstoy, 1991, pp. 479).

In this extract, phenomenal qualities are described by means of propositional attitudes, whilst causal events are described according to theoretical propositions. Previously, I have argued that any formal theory of organisational functioning must cohere with our best theories of human learning. The same may be said of leadership. However, our best theories of learning are those that are conceived naturalistically by the cognitive sciences. Whilst that continues to be the case, phenomenal perceptions of leadership may only cohere with our best theories of learning if phenomenology can be naturalised - and that is a project that is yet to be realised (De Preester, 2006).

A similar example can be seen in a comment made by the School Council President of Eucalypt Primary School about school vision:

A large part of the school community would not have a vision. They would be happy to go along with the decisions made for them by the school. But on the other hand, there is a group of parents who have a strong vision; it's an environmental vision. They would like to have hundreds of thousands of dollars spent on the actual outside school environment. But at the same time, they are very driven; passionate, articulate parents who need to get part of their vision because that motivates them and keeps them passionate. So within the bounds of the money that we do have, that we can free up, we've given that group of parents quite a substantial amount of money linked to the school environment; and we'll be giving a bucket of money to them at the end of next year too, to do some more things (Interview B6).

In another example concerning leadership, the Acting Assistant Principal of Eucalypt Primary School, said,

I enjoy the acting position of Assistant Principal and I took it on as a challenge, but I'm not sure that it is something that I would apply for in another school. It's more, for me, a learning curve. So, when I took on the acting position, what I wanted to achieve were the tasks I was given, I'm very much a person who wants to start and finish a task. If I'm responsible for PD, I want to make sure that it's done properly. Assessment and reporting: If I have to devise a new report format then I do that and then implement it. So, I want to make sure that I do the 
job that I'm given by the principal and those sorts of tasks. I guess the other thing was to be seen as a leader in the school. When I first took over the position, it was a jump from a level one teacher - a level one teacher to an AP is a huge jump! (Interview B8).

The above extracts illustrate how propositional attitude sentences may be placed alongside causal-computational theoretical propositions within the one account. It is in this way that leadership and organisational functioning are conflated. As practitioners talk about their practice, they tend to talk about the intentionality of experience as much as the nature of events, especially in leadership narratives.

Leadership narratives employ a variety of culturally mediated schemas. These include the schemas of leadership-as-competence, leadership-as-influence, leadership-as-political-action, leadership-as-image, leadership-as-social relations, leadership-as-performance, leadership-as-innovation, and leaderless innovation. Schemas such as these are employed in situational narratives, which emphasise the particular circumstances and details of the situation over the generalities of theory. In contrast, grand narratives seek to generalise by reducing the particularities of individual situations to a single theoretical account. The richness of situational narratives resists generalisation, however, because they employ propositional attitude sentences, which are structurally ambiguous.

Attempts to generalise leadership within formal leadership theories have suffered from problems of definition and lack of predictive power. This problem stems in large part from the emphasis leadership theories give to the actions of individual people, and the distinctiveness of individual situations:

There are no guarantees that a particular leader will be effective by adopting a recommended approach! Leaders are too different in preferences and personality, and leadership contexts are too different in their scope and contours for leadership to be so neatly packaged. Even if leaders do the same things, the things don't turn out to have the same effects. No single strategy, style, list, or formula fits all situations in the same way (Sergiovanni, 2001, p. 20).

Whilst a phenomenal perception of leadership might sit comfortably within the special cases described by situational narrative, it is certainly not amenable to global 
generalisations in the guise of formal, rule-governed explanations. Phenomenal descriptions of leadership are highly descriptive, informal and context-dependent. Whilst human cognition and organisational functioning may be described in causal terms, leadership may only be described in phenomenal terms.

Both causal-computational explanations and the phenomenal perception of quality incorporate human emotions and culture in their accounts. However, one examines emotions and culture from an external perspective, whilst the other takes an internal perspective. The external perspective focuses on the mechanisms and outward manifestations of emotions and culture in dispassionate terms, whilst the internal perspective focuses on the feelings and experiences of the individual and personal perceptions of value. Whilst one describes emotion and culture in terms of quantitative measures of cause and effect and the computations of parallel distributed processing systems, the other describes them in terms of the propositional attitudes of hope, belief, and purpose and the value of perceived qualities.

Distributed cognition theory utilises causal or computational explanations for organisational functioning, according to which leadership may be described as an emergent, self organising property of a complex system (Lakomski, 2005, p. viii). According to Ellis (2005), causal effects can arise from non-material features such as concepts, information and purposes, and those features are ontologically real. The strong reductionism that characterises the science of physics denies the ontological status of such claims; but Ellis holds the view that a commitment to emergence entails a theoretical commitment to those features:

\footnotetext{
Emergence takes place in, and partly enables, a context of multiple natures of existence relating to (a) particles and fields (the material world), (b) possibility landscapes characterising possible existence and changes of state (controlled by the laws of physics), (c) human ideas, goals and intentions, emotions, and social constructions, and (d) platonic mathematical properties and objects (Ellis 2003).
}

Since leadership is a product of human ideas, goals, intentions, emotions and social constructions, it is amenable to description as an emergent property. 
Furthermore, the intentional character of goals and purposes also renders leadership as a phenomenally perceived quality.

Just as the perception of colour is affected by colour prototypes, background effects, lighting and so on, perceptions of leadership may be affected by the means of perception, including heuristic, schema, prototype or attribution; and by the background presented by artefacts, culture and environment. Consequently, leadership prototypes may be vulnerable to attribution bias due to the background properties of different cultural contexts. This may explain why leadership prototypes differ significantly from culture to culture (Popper, 2004). Even within a single culture, leadership schemas may differ considerably from individual to individual so that where one person perceives 'strong leadership', another perceives weak or absent leadership. The propagation and magnification of leadership perceptions may arise from the complex social dynamics arising from cultural background factors, the emotional responses of individuals, and communication of perceived attributes in terms of propositional attitudes.

Hence, leadership may be conceptualised as a relationship between individuals perceived in time and place as a phenomenal complex:

\footnotetext{
A true picture of a phenomenon so complex as leadership cannot be obtained, to use a metaphor from photography, by looking at stills, which freeze a situation at a given moment; it requires photography that shows movement over time (Popper, 2004, p. 118).
}

When viewed in this way, a contemporary notion of leadership may be understood as a relation, perceived in the light of qualitative judgements about the interpersonal relationships of formal positional leaders and followers. A school principal, for example, may be said to be 'exercising leadership' or 'not exercising leadership, or exercising 'more' or 'less' leadership than another principal, depending on the perspective of the observer. Alternatively, the perceptions of observers may result in a formal positional leader, such as a school principal, being paradoxically labelled as 'not a leader', whilst a person in the school who does not hold a formal leadership position may be labelled as 'a leader'. 
Many conceptions of formal positional leaders hold connotations of power, such that the leader has power over followers. This view of leadership has its roots in the exercise of power of masters over slaves or kings over subjects. If this were all there was to leadership then leadership would simply be a matter of one person exerting his or her will over others so that, followers fulfil the will of the leader because they are compelled to, not because they choose to:

Those he commands move only in command, Nothing in love.

(Shakespeare, Macbeth, Act 5, Scene 2, Lines 19-20a).

Whilst Shakespeare and his contemporaries acknowledged that leadership entailed the exercise of power over others, the consent of followers was considered important too. In this way, leadership is not admitted by leaders alone, but also by followers who choose to accede their powers to a leader. Though, if leadership were solely a power relation the follower would have no power to choose at all, logically speaking. However, the folk psychological view is that power relations are seldom absolute. In Shakespeare's Othello, for example, Iago chooses to follow Othello for his own ends:

I follow him to serve my turn upon him:

We cannot all be masters, nor all masters

Cannot be truly followed.

(Shakespeare, Othello, Act 1, Scene 1, Lines 42-44a).

Whilst human cognition, as described by distributed cognition theory, entails both causal and computational relations, distributed leadership must also admit purposeful, free-will choices.

\subsubsection{Narrative meaning}

Purposeful, free-will choices are described in terms of the propositional attitudes and non-literal phrases of folk psychology. In contrast, deterministic, causal relations are explained in literal terms by means of formal theoretical propositions. The values, tacit knowledge, and cultural influences that give rise to the propositional 
attitudes of folk psychology may be understood as informal theoretical constructs. As the accounts of folk psychology interact with the causally justified descriptions of science, some commonsense theories of folk psychology are changed, whilst others remain stable. The inductive character of first-person perception and the reinforcing character of culture may account in part for the persistence of folk psychological theories. In addition, our inability to gain direct access to the 'inside-looking-out' perceptions of others, and the ambiguity of the propositional attitudes of intention and belief, render folk psychological theories about the intention of others opaque to reliable analysis and criticism.

The understanding of leadership that we obtain from leadership narratives and from the narratives of day-to-day life arises from culturally and linguistically mediated information. Propositional attitude sentences typically provide literal interpretations of the propositional attitudes of belief and desire. However, a great deal of communication in day-to-day life occurs through non-literal means. In narrative, attributions of leadership can be conveyed through non-literal metonymic expressions, such as in the phrase, 'Hitler invaded Poland'; which in this case literally means that armed forces, under Hitler's command, invaded Poland.

The complexity of non-literal language forms is evident in expressions such as, 'The principal improved the tone of the school'. In this case, the phenomenal perception of a difference of 'tone' is combined with the synecdochical attribution of cause to the principal. The term 'principal' literally refers to a person occupying a particular place in the organisational structure of a school. In a non-literal sense, however, the term 'principal' may also stand in place of the complex interplay of organisational members ${ }^{83}$. When the term 'principal' is interpreted non-literally as synecdoche, the original sentence becomes, 'The school improved the tone of the school'. Hence, the literal construal of the statement gives rise to a tautology, which

\footnotetext{
${ }^{83}$ This is a case of synecdoche similar to the phrase, 'all hands on deck', where the term 'hands', which literally refers to the hands of crew members, also has a non-literal meaning that refers to the crew members themselves. In this case, the term 'principal', who is literally a part of the school, stands in place of the other members of the school community.
} 
retains the phenomenal quality of 'tone' in the predicate but loses its force as a causal-computational explanation.

One of the ways cognitive science has endeavoured to explain human understanding is by investigating metaphor (Schleifer, Davis and Mergler, 1992, pp. 43-51). In classical times, the role of metaphor, in determining meaning, was explained in terms of binary oppositions. However, non-literal expressions employ senses in complex ways and a variety of forms, including anthimeria, banter, euphemisms, hyperbole, idiom, irony, litotes, metaphor, metonymy, personification, sarcasm, simile, synecdoche, meiosis, onomatopoeia, oxymoron, paradox, pun, and understatement. The meanings of non-literal language forms are perceived meanings and therefore have phenomenal character. It is in perceiving similarities and differences that we attain phenomenal understanding. Metaphors provide a means for abbreviating knowledge and supplement insight through the infiltration and dispersion of concepts, and culminate in an emotionally affected climax. The use of metaphor is a cognitive process involving the introduction and expression of concepts, and the cultural transmutation of language. Metaphors may act as cognitive organisers, activating existing schemas or prompting the formation of new schemas in thought and culture.

The interpretation of non-literal language and narrative requires the resolution of binary opposites (Schleifer, Davis and Mergler, 1992, pp. 49-63). The interpretation of metaphor, for example, may entail three stages of judgement. Firstly, the binary opposites of truth and untruth enable the recognition of obvious truth. Secondly, the binary opposites of sense verses nonsense enables a literal interpretation of metaphor to be classified as nonsense; which leads in turn to the consideration of further information by the contextualisation and recontextualisation of meaning. This view resembles a narrative drama by virtue of its "narrated stages of simple and complex binary oppositions' (Schleifer et al., 1992, p. 50). The opposition of truth and falseness, and sense and nonsense requires that information be marked with more information and situated within a particular contextual framework. In this way, a crisis of meaninglessness may be circumvented. This resolution of unpalatable 
meaninglessness lies at the heart of humour, which serves to release the tension of a situation and endow it with meaning.

A binary opposition also occurs in the conflict of cognitive and non-cognitive conceptions of understanding, and the opposition of apprehension and narrative as appears in the conflict between story and argument. This is what Bruner (1986, pp. 11-12) portrays as the conflict between the 'narrative mode' and the 'paradigmatic or logico-scientific mode of thinking'. The first is phenomenal in character, sensitive to the situation, and comprehensible only to those who have had similar experiences; the second is causal in character, universal, and amenable to interpersonal standardisation. The opposition of these two modes of thought, according to Schleifer, Davis and Mergler (1992, pp. 58-63), leads to formalisms, which in their view, attempt to

\begin{abstract}
recuperate, modally and functionally, the phenomenological and temporal processes of apprehension and organisation. Like the opposition of presence and absence in the distinctive features of phonemes, they allow the phenomena studied to exist as phenomena [italics in original] both in time and in cognitive apprehension. In this, they allow for logical structures and a plurality of cultures ... For this reason, phenomenal meaning-effects exist and function as narrative units because logical organisations are apprehended as real temporal events - cultural events (Schleifer, Davis and Mergler, 1992, p. 62).
\end{abstract}

Hence, phenomenal meaning draws on cultural events to function narratively. According to Katz (2005a), little systematic progress has been made in the systematic analysis of the social and cultural factors of language processing, especially non-literal language. In contrast to the research program of Chomsky (1957), Katz (2005a) suggests that the study of non-literal language sheds light on the effects of social and cultural knowledge. Non-literal language is an important component of situated narrative, and of folk-psychology, because it expresses the speaker's intentions beyond what is being expressed in literal terms. That is, nonliteral language relies on a paradoxically non-sentential characteristic of sentences, a characteristic that may be explained partly, but not entirely, by the representative capabilities of parallel distributed processing. 
The occurrence of both literal and non-literal interpretations of language arises because language is inherently undetermined. Comprehended meanings extend beyond literal language meanings due to processes that include referential assignment, the resolution of ambiguity and the generation of inferences (Colston, 2005, p. 99). This is true of all forms of language, and indicates that there is more to phenomenal human experience arising from human cognition and human experience than can be expressed in words (in a literal sense). The connection between nonliteral language forms and folk psychology is evident in Colston's (2005) reference to on-line and off-line language processing modes. This is similar to the distinction Gordon (1995) makes between on-line and off-line theories of folk-psychology.

\subsubsection{Leadership in information space}

We humans process information through our interactions with each other, our environment and the artefacts we have created. This includes the off-line simulation of the cognitive processes of others and the on-line processing of environmental information. Our practical dealings with the world consist of being there (or Dasein, after Heidegger, 1962) so that the very act of being requires that we interpret our contextual relations to things in the world (Smith, 2005). It is in the light of this phenomenological conception of being that leadership obtains meaning.

The phenomenal character of leadership poses problems for its analysis within a naturalistic epistemology. The tension between naturalistic and phenomenological world views may be ameliorated by adopting a form of property dualism. Phenomenological research methods cannot replace naturalistic methods as a means to understanding the objective world. However, phenomenology may reveal the essence of leadership as perceived by organisational participants through their lived experience (Ehrich, 2005). Hence, a naturalistic account of organisational functioning may stand alongside a phenomenal account of leadership. This allows the causal-computational account of distributed cognition to be complemented by the intuitive experience of leadership as expressed through the intentional categories of phenomenology. 
If phenomenological accounts of leadership cannot be naturalised, can they play any part in a naturalistic study? The answer, I think, is yes - and I offer two reasons for this stance. Firstly, the blurring of the distinction between fact and value renders the value-laden accounts of folk psychology amenable to observation as features of human learning and culture. This is not equivalent to the naturalisation of phenomenology because as De Preester (2006, p. 59) argues, while it enables phenomenology's descriptive results concerning consciousness and subjective experience to be naturalised, it does not amount to the naturalisation of the transcendental attitude of phenomenology. Secondly, as Chalmers (1995, p. 152) has argued, explaining human intentions as manifestations of consciousness may require that both causal and phenomenal properties be summoned to that purpose. His argument is that phenomenal properties and physical or computational properties share a common information space. If so, then phenomenal properties may be considered in parallel with naturalistic properties. It follows that distributed leadership theory includes both causal and phenomenal properties, with organisational functioning being described naturalistically and leadership being described phenomenologically.

Attempts to capture leadership in causal terms may be seen in The Developmental Learning Framework for School Leaders (2007). Take the following example, which specifies criteria for judging a high level of educational leadership behaviour:

\begin{abstract}
Leaders ensure common models of learning and teaching underpin all classroom practice. They ensure that the principles of developmental learning inform the alignment of curriculum, pedagogy, assessment and reporting. To improve learning outcomes, they verify that students and staff self-evaluate against goals and targets. Leaders promote further improvement by systematically collecting evidence of how reflective practices contribute to improvement in teacher practice. They influence curriculum practices in other schools and design initiatives that build the capacity of people across the system (The Developmental Learning Framework for School Leaders, 2007, p. 7).
\end{abstract}

This extract describes educational leadership in criterion-referenced terms. The use of such descriptors requires that their sentences be interpreted and matched to remembered first-hand observations or the third-hand reports of others. First-hand 
observations are interpreted according to the background dispositions and theories of observers and matched to a complex of phenomenally perceived observations, filtered by theory and stored in memory.

The above example assumes that leaders can know the minds of teachers to the extent that they know whether teachers are employing a 'common model of learning and teaching'. This entails knowing the schemas underlying the statements that teachers utter as well as the identification of intentionality in teachers' observable actions.

As argued in previous chapters, self-evaluation cannot be a computational process. Judging the outcome of teacher actions requires the evaluation of purposeful action as declared by means of structurally ambiguous propositional attitude sentences. Criterion-referenced statements of the sort shown above assume it is possible to read and interpret the minds of others, therefore assuming an unstated theory of mind. In the circumstances of unequal power relations, one opinion may be valued over another because a person in a position of authority (e.g. a principal) has more institutional power than another (e.g. a teacher).

The expectation that principals can verify that students and teachers are selfevaluating their performances against goals and targets, seems to assume that they can judge between purposeful behaviour and behaviour conducted by compulsion or according to deterministic rules. Given the structural ambiguity of the propositional attitudes, attempts to verify such a thing will be subject to chance. Phenomenal perception is based on the limitations of first-person observations and the information conveyed via second or third hand observation reports. Given that, all observation is theory-laden and we lack the ability to explain first person phenomenal perception satisfactorily, a lot depends on the manner in which this information is obtained and the means by which it is checked and interpreted.

The 'alignment of curriculum, pedagogy, and assessment and reporting', referred to in the above quotation, entails a phenomenally complex perception of the synchronisation of a huge number of individual actions, many of which cannot be 
directly observed in the normal course of school life. Certainly, one person could not possibly make direct observations of the myriad actions that take place in a single school day let alone longer periods (except perhaps in the case of a very small school). If a school leader was to rely on second and third hand accounts, then he or she could be accused of making performance judgements based on hearsay and innuendo. Though it might be possible to observe a small portion of school life by employing technology-assisted ethnographic research methods (such as the methods employed by Hutchins, 1995, and Clarke, 2004), that is an extraordinarily difficult and impractical thing for school leaders to do in the course of normal school life.

The Developmental Learning Framework for School Leaders (2007, p. 7) relies heavily on judgements about intentions, intuitions, and emotional awareness. The framework leaves principals room to judge causal-computational effects by reference to cultural customs, the use of resources, the expertise of staff members, school processes, and the transfer of learning between schools. However, the high level measures of the document are described solely in terms of propositional attitudes. Causal-computational effects are downplayed as indicators of low to mid level leadership capabilities. The framework's stated adherence to transformational leadership theory, interprets that theory in terms of the actions of the individual principal. Little acknowledgement is given of the leader's dependence on his or her followers or the characteristics of the situation. Despite its stated acknowledgement of distributed leadership, the framework is phrased almost entirely in terms of the autonomous actions and efficacy of the individual leader.

\subsubsection{Distributed cognition}

Unlike traditional leadership theories, such as transformational leadership, I argue that organisational functioning is satisfactorily explained according to distributed cognition theory. According to this theory, humans actively structure and utilise the 'contours' of their environments. As they do so, they reduce the cognitive work required for individuals to fulfil their physical needs and participate in the production and reproduction of social life. Distributed cognition is a causalcomputational account that is 
closely tied to the idea of automated information processing and the mechanistic transformations of representations. According to this kind of account, we would find computation whenever we found a mechanistically governed transition between representations, irrespective of whether those representations participate in a representation scheme that is sufficiently detailed to count as a stored program (Clark, 1997, p. 159).

Information becomes knowledge when it is represented in individuals in ways that refer to the outside world. Knowledge may be formalised in terms of generally applicable theoretical propositions or rules that enable a theory's explanatory adequacy to be compared with competing theories. Theoretical propositions describe the content of theories in sentences, which obtain their meaning by reference to other trusted theoretical propositions and by reference to the implied or tacit knowledge of the knower. Hence, knowledge may be held by individuals in the patterns of cognitive schemas, and within culture as behavioural norms and values. Knowledge may also be embedded in artefacts, built into their physical structures and held as semantically coded information. Knowledge also exists through the way humans draw on tacitly known experiential knowledge to conceptualise, negotiate and shape their environments.

Since this view stretches information space across mind, body and world, human knowledge becomes distributed so that it is no longer understood as knowledge that is localised in the brain. Information does not become knowledge until it refers to the objects of knowledge, which are in turn known by reference to other objects. Hence, distributed cognition, as its name implies, distributes knowledge across brain, body and world.

Although an organisation can be fruitfully described in terms of the computational processing of information within and between people, artefacts and environment, there is, nevertheless, more to an organisation than computation:

An important aspect of the larger unit is that it contains computational elements (persons) who cannot be described entirely in computational terms (Hutchins, 1995, p. xv). 
Distributed cognition explains organisational functioning in formally causal and computational terms. Yet it also allows for the description of the relationship between culture and human cognition in organisational action. Hutchins (1995, p. 203), speaks of quartermasters in the Standard Steaming Watch of a ship as computational elements who 'align themselves as a coordinating structure that passes information from one transforming device to another'. Here the instrument readings lessen the cognitive load on the quartermaster whilst he coordinates his actions with the instruments, which are here referred to as 'representational technologies':

The formal relationship is one of spatial correspondence. The causal relationship is a tissue of human relationships in which individual watchstanders consent to have their behaviour constrained by others, who are themselves constrained by the meaningful states of representational technologies (Hutchins, 1995, p. 202).

Similarly, distributed cognition theory can provide a useful framework for the analysis of student conceptual learning processes in schools, especially concerning the use of learning technologies (Connolly, 2006; Valanides and Angeli, 2008; Edmondson and Beale, 2008; Angeli, 2008).

\subsection{Distributed Leadership}

The phenomenal character of leadership works against attempts to construct a context-free definition of 'leadership'. Numerous definitions have been assembled and none can be applied universally to all situations (Hughes et al., 1995, pp. 41-2; Karmel, 1978, p. 476). The adequacy of any one definition of leadership will depend on the situation to which it is applied. Since no knowledge can be foundational, there can be no foundational principles of leadership. Leadership has no unique physical characteristics and the qualities associated with leadership will be perceived differently by different people and in different situations. Neither are there any physiological states of emotion, associated with the motivation of an individual, which may be uniquely attributed to leadership. A decision to attribute a particular emotional state to leadership rather than some other cause owes more to disposition 
and theory than to observation. Leadership, as a concept, is not amenable to causal explanations that may be repeatedly confirmed by observation.

In contrast, distributed cognition theory acknowledges that causal explanations for human cognition and action may be given in terms of direct and indirect, proximal and distant, or strong and weak causes. The computational aspect of cognition allows direct causes to arise from indirect causes, for proximal causes to arise from distant causes, and for an accumulation of weaker causes to give rise to stronger causes. Since the same decisions or actions applied to different contexts and situations typically produce different results (Sergiovanni, 2001, p. ix), leadership cannot be satisfactorily described in terms of the actions of the leader alone. What leadership theories often attempt to do is to reduce leadership to the decisions and actions of the leader without considering the context in which the leader works. Nevertheless, that context or situation may be the source of indirect, distant or weak causal factors, those factors may have significant consequences.

Situation theories of leadership do consider context but also ignore the interactions of the people working in those situations. They assume that situations and leadership styles remain static so that a deterministic relationship exists between situations and the behaviours required for leadership. Contingency theories attempt to combine the better characteristics of the trait and situation theories, but remain overly deterministic. That this deterministic character is viewed optimistically is more indicative of the commitment of researchers to a logical empiricist epistemology than the model's theoretical validity. Although meta-empirical studies indicate broad statistical support for contingency theory, the model suffers from difficulties in applying it to non-laboratory studies, and its failure to deal with the complex detail presented by the dynamic interactions within organisations (McMahon, 1972; Strube and Garcia, 1981; Peters, Hartke and Holman, 1985).

Sergiovanni (1992) appears to accept some of the tenets of contingency theory when he argues that direct leadership is only necessary in the absence of a sense of community. However, he also accepts a phenomenological interpretation of leadership, one that refers to intentionality. Direct leadership entails the authoritative, 
forceful, direct and interpersonal actions of a person labelled 'the leader'. Alternatively, indirect leadership is a process of internal value and motivation, shared by the members of a community. With indirect leadership, values, emotions and social bonds bond a community, so that people are motivated by what they consider to be right, just and good, and projects to which they feel a sense of obligation and commitment. In a community, as described by Sergiovanni (1992), things are done because they are intrinsically rewarding, not because people are closely supervised and given external rewards. Hence, Sergiovanni's construal of direct leadership is described in causal-computational terms, whilst indirect leadership is described in terms of propositional attitudes.

Distributed leadership also acknowledges the cognitive ties between people as mediated by their cultural and emotional commitments. It acknowledges the importance of environment, culture and artefacts to human cognition and allows for a view of leadership that transcends the causal actions of formal positional leaders (Spillane, 2006, pp. 1-27). In this view, the causal efficacy normally ascribed to formal positional leaders is reconceptualised as the outcome of interactions between people and situations. Leadership can only complement causal processes insofar as organisational members assume its existence and motivational relevance. Hence, what distributed leadership offers is a culturally mediated perception about the quality of organisational events. Perceptions of leadership qualities can differ in significant ways from individual to individual, from culture to culture, and from organisation to organisation.

Viewed as causal-computational interactions, even though the details of organisational activities may differ from situation to situation, they share a common set of underlying causal and computational mechanisms. Hence, human cognition and therefore organisational functioning may be explained according to formal, causal-computational rules, but leadership cannot. Leadership, in whatever form it is conceived, is only significant when it is culturally significant. Since cultural factors may vary from situation to situation, it follows that the significance of leadership will vary from situation to situation. 
If however, a situation were to occur in which cultural norms do not include some version of leadership, leadership could not figure in explanations of organisational functioning. In such cases, organisational innovation emerges from the dynamic interactions of individuals, artefacts, culture and environment. Such situations, which include Hutchins' (1995, pp. 1-6) account of a bridge crew of a naval vessel as they interactively solved a pressing navigational problem, are not necessarily accounts of leadership or distributed labour (see Lakomski, 2005, pp. 7172). Even though a rigidly defined organisational structure exists on a naval vessel, and entails a formal division of labour, Hutchins' example demonstrates that the solution of novel problems does not necessarily depend upon the prescription of leadership that is implied by such formalisms.

\subsubsection{Distributed leadership: prescription or description}

Gronn (2002a, 2003) identifies the tendency to prescribe rather than describe leadership as a deficiency in orthodox leadership theory, particularly when divisions of labour undergo change in the workplace. He argues that distributed patterns of leadership have emerged from changes in organisation due to the addition and modification of work tasks and the introduction of new technologies.

In putting forward his taxonomy of leadership, Gronn (2002a, p. 441) cautions against assumed connections between management and leadership, as leadership may be attributed to any individual or concertive unit within an organisation. Causal relations between managers and subordinates are governed by employment contracts that preserve disjoint authority relations and incline employees' towards compliance with managers' directives. Phenomenological perceptions of leadership, however, may strengthen the causal-compliance relationship. That is, when employees regard their managers as 'good' leaders, that entails an emotional commitment to their managers, which might be interpreted as having causal outcomes in the form of 'value-added' contributions to the organisation. Conversely, if they do not regard their managers as leaders, they may limit their actions to compliance. Gronn suggests that a working knowledge of distributed leadership as conjoint agency and concertive 
action may contribute to successful learning from training and a better understanding of the realities of workplace practices.

This mixing of causal-computational and phenomenological explanations presents a number of difficulties. Firstly, intentions are not amenable to naturalistic analysis. If a particular intention is said to cause a particular outcome, and that intention is said to be caused by some sort of disposition (say, of genetic or cultural origin), then it may be argued that the follower had no choice in the matter and that the appearance of intentionality is a mere illusion. Hence, the attribution of intentionality, and therefore of leadership, appears to add nothing to naturalistic explanations about the functioning of organisations. However, if a phenomenological explanation of leadership is placed alongside a causal-computational explanation of organisational functioning, rather than being integrated into the one account, this problem is diminished. The two accounts intersect only to the extent that they describe the same information space and enable philosophical triangulation to take place. The phenomenological account simply describes organisational life from a different standpoint to that taken by a naturalistic account. Whilst the former may be normative and prescriptive, the latter is descriptive and explanatory.

\subsubsection{Cognition, culture and place}

In a naturalistic analysis, culture reinforces the cognitive schemas that individuals hold by repeating and reinforcing dominant patterns of behaviour. This reinforcement evokes varied emotional responses from individuals because the motivational force of a cultural schema will differ from individual to individual (Strauss and Quinn, 1997, pp. 89-110). The distributed nature of cultural knowledge and norms arises from the interaction of the mental models of individuals with 'variegated public representations'. Hence, culture is not legitimately understood 'as a single kind of thing' (Strauss, 1997, p. 251).

Hutchins (1995) emphasises the unique contribution of the inter-psychological functions of culture in distributed cognition: 
Because society has a different architecture and different communication properties than the individual mind, it is possible that there are interpsychological functions that can never be internalised by a single individual. The inter-psychological level has properties of its own, some of which may not be the properties of any of the individuals that make it up (Hutchins, 1995, p. 284).

Whilst each individual stores information in cognitive schemas, the information held by culture, emerges from the interactions of many individuals. That is, the information held by culture supervenes over the information held by any one individual, so that the information held by the culture is greater than the simple sum of the information held by individuals.

The phenomenologically distributed nature of culture is starkly evident in the African cultural concept of ubuntu, which may be translated into English, as 'I am a person through other persons ${ }^{84}$. Malcolm (2003) describes the importance of ubuntu to student learning in South African schools. Ubuntu extends the concept of personhood to objects in nature:

In ubuntu, all things are "persons", but some more than others. A river is more person than a rock is, an animal more than a tree, an adult more than a child, an ancestor more than an old man. While relationships with nature are important, relationships with people and ancestors are more powerful, and deserve greater respect. Further there are authoritative people in the community who warrant special respect, such as healers, chiefs and teachers' (Malcolm, 2003, p. 36).

This concept emphasises the interaction of the individual with others in a way that renders the individual inseparable from others. Hence, the individual is expressed through others and others are expressed through the individual. Since it is possible for an individual to have connections with more than one community, it is also possible for an individual to have many selves, each self governed by particular relationships.

A similar theme has emerged in discussions about the education of indigenous Australians. Much has been said about the value of recognising indigenous culture,

${ }^{84}$ As Levinas (1973, p. 51) says, the notion of self presupposes intentionality. 
to redress the injustices of past conflicts between indigenous and white Australian cultures. This view achieved recent prominence when Prime Minister Kevin Rudd read, in the Australian Parliament, an apology to the 'stolen generations' of indigenous people (Parliament of Australia: Hansard, 13 February 2008). Australian schools (in the same time zone) paused to listen to a live broadcast of his speech.

Also important to Australian indigenous culture are the concepts of song and space. Songs and chants are an essential part of the indigenous oral tradition and form the mythological-historical basis of their view of the world and their sense of self (Fell, 1999). Each individual in indigenous culture derives identity and knowledge from the experience of singing. The history and laws of the community are taught, the individual is nurtured, the well-being of the group is protected, and places are named through song. Singing is important to Australian indigenous thinking because it defines the norms and essential patterns of culture and conveys culturally important notions of time, place and self.

Whilst it may be conceived phenomenologically, a situated nature of human cognition is also evident in the concept of 'place'. As we project our schemas onto defined spaces, we contextualise them to make them places:

Place - the way we conceptualise it, the way we organise it, the way we temporalise it - forms a core part of our understanding of the world around us (Saab, 2003, p. 1)

Spatial knowledge includes both declarative and procedural knowledge (Saab 2003), whereby declarative knowledge is that obtained by cultural means such as by storytelling, reading or schooling. This type of knowledge is represented by abstract geographical statements such as 'Canberra is the capital of Australia'. On the other hand, procedural knowledge is the experiential form of spatial knowledge by which associations are formed between particular natural features, artefacts and events so that they constitute a situation.

A situation is much more than a context that mediates leadership practice, as argued by contingency theorists such as Fiedler and Garcia (1987). According to 
distributed cognition theory, people interact with situations in complex ways so that any one situation overlaps with many other situations constituted by history, culture and artefacts. The level of analysis at which a situation is defined and the characteristics that are deemed part or not part of a situation give rise to innumerable ways to define and conceptualise a given situation.

Consequently, defining a situation may involve the attribution of causal relevance to the various elements of a situation and their interactions. In addition, a situation may shape the actions and thoughts, intentions and emotions of people as they imbue it with meaning. Situation both shapes and is shaped by the interactions of people:

Distributed leadership views the situation in interaction with leaders and followers as an element that mutually constitutes leadership practice. In this view, the situation does not simply affect what school leaders do; in interaction with leaders and followers, the situation defines leadership practice (Spillane, 2006, p. 22).

The identification of leaders and followers in a given situation arises from phenomenological perception. Since leaders and followers are 'two sides of the one coin', any leadership process will require the participation of followers as much as leaders. The phenomenological perceptions of the followers, their consent, and the intentionality of their participation are important to the categorisation of a person's actions as 'leadership'.

\subsubsection{Cognition, leadership and language}

Rhetoric is an important component of situation that is associated with the qualities of leadership. Wreden (2005) identifies Winston Churchill as a leader who not only won the Nobel Prize for Literature in 1953, but who is also credited with rallying the British people during the Second World War. Similarly, Frank Tate, Director of the Victorian Education Department (1902-1928), is remembered for his visionary disposition and skills as an orator:

Contemporaries remembered Tate as a tall young man with dark curly hair, a powerful and attractive personality, marked skill as a raconteur, an 
agile wit, a passion for literature and an overwhelming determination to succeed (Selleck, 1990).

According to Wreden (2005), the skills of motivational oration in leadership include a careful choice of words, appropriate rhythm and cadence, careful assembly of data, use of metaphor and analogy, and the emotional engagement of speaker and audience. The flexibility and rhetorical possibilities of language can be underestimated by conceptual and semantic analyses (Edwards, 1997, pp. 170-201), because

\begin{abstract}
the rich variety of emotion metaphors, and empirical studies of discourse, suggest something more like a set of rhetorical affordances, in which different part or potentials of meaning, even contrasting ones, may be worked up and deployed, on and for occasions. This conceptual and rhetorical flexibility, although plainly a nuisance to semantic and cognitive model-making, is ideal for the action-performing, accountability-oriented, rhetorical 'witcraft' of discourse (Edwards, 1997, p. 193).
\end{abstract}

The appeal of rhetoric as a means of enacting leadership lies in a sense of shared human experience to which the orator and audience can relate. That sense can be communicated through both literal and non-literal forms of language; however, nonliteral language forms lend themselves more readily to that purpose (Link and Kreuz, 2005, pp. 156-157). In literal expressions, the same words may be used to express both emotions and attitudes (Young, 1943, p. 65) and the propositional attitudes may be understood as dispositions towards particular emotional states. In non-literal expressions, emotion and attitude are expressed through whole sentences or passages utilising forms such as metaphor, simile, idiom, hyperbole, understatement, and irony. Link and Kreuz (2005, p. 157) suggest that non-literal language is more suitable than literal language for distinguishing the varying intensities of an emotion. For example, when comparing the non-literal statement, "She was like a kid in a candy store", with the corresponding literal statement, "She was very happy", the former may convey a stronger sense of happiness than the latter.

Whilst a distributed leadership differs from the classic notion of a leader as an extraordinary, heroic individual whose actions causally transform an organisation, it 
leaves room for the analysis of emotion from both naturalistic and phenomenological viewpoints. Instead of explaining organisational change in terms of the autonomous actions of an individual, a distributed view frames human learning within communities of practice. It acknowledges that human cognition and learning may be understood as a distributed causal-computational process, whilst leadership may be understood as the intentional, first-person perspective of those who participate in organisations.

\subsubsection{The distribution of leadership}

The concept of distributed leadership is constituted as a 'web' of leaders, followers, and situation (Spillane, 2006, p. 3). Each of these three elements may be judged according to phenomenal qualities. Together, they constitute a leadership complex that differs as the three elements differ. A person may be perceived to be a 'leader' or a 'follower' in some situations but not in others in accordance with preexisting expectations of leaders, or followers, as perceived against a background of particular states of affairs. A social or organisational situation may also be perceived in different ways - in terms of perceived physical or temporal boundaries, social norms, historical precedent, classification, and so on. For example, a school classroom may be perceived in relation to the wider environment of the school, cultural expectations, interpersonal relations of students, actions of the teacher, temporal location of the class on the school timetable, physical environment of the classroom, and so on. Focusing on any one of these aspects, or any particular combination of these at the exclusion of others, changes the character of phenomenal experience.

Distributed leadership is also the outworking of distributed cognition. Whilst delegation of organisational responsibilities in accordance with the principles of division of labour may be a defensible outcome of the problem-solving capability of human cognition, the version of distributed leadership presented in this thesis is not merely a process of delegation, or role specification. This view is consistent with that described by Spillane (2006) when he says: 
Distribution of school leadership responsibilities across leaders does not arise solely through public decrees or private decisions of school administrators. Distributed leadership is not just delegated leadership (Spillane, 2006, p. 13).

The 'leader-plus' view of leadership discussed by Spillane (2006, pp. 12-15) recognises that many people enact the functions and initiatives of a school. It also recognises that all people, including those labelled as leaders, bear their actions up on a scaffold of organisational norms, cultural expectations, historical antecedents, and the like. Much of what people do arises from collective, interdependent, and interactive actions, which occur in response to a given situation, but which also manipulate and transform the physical environment, social structures and artefacts that make up the situation.

There is a tendency for formal leadership theories and the informal portrayals of leadership found in popular books to focus on individuals who have been labelled as leaders. Those labels most often correspond with the formal designations of organisational structures; however, they may also be attached to individuals without formal title, but to whom some extraordinary change or other is attributed. The distributed leadership practice envisaged by Spillane (2006) cannot be attributed to individuals but is stretched over 'multiple leaders' and is therefore 'co-enacted' (Spillane, 2006, p. 15). In this view, leadership practice takes place between leaders and between leaders and followers, and is therefore not located in any one individual.

The interactions of individuals constitute routine and novel actions, often mediated by artefacts. For example, the posting of a meeting agenda on a local intranet may stimulate pre-meeting discussion about the agenda via email, telephone or within a quiet corridor. The history and character of these artefacts may engender a more informal discussion. As objects that were created within human culture and for historically constituted purposes, these artefacts carry cultural capital. Taking the example further, when the meeting takes place, the discussion may follow formal cultural conventions that are accentuated by a venue that engenders formality; such as a formally titled 'conference room' containing artefacts associated with a culturally constituted notion of ritual. 
Any situation of human social or organisational action comprises a physical environment, cultural capital and artefacts. The physical environment itself is likely to be composed of both natural and artefactual objects. Cultural capital comprises social structures, routines and artefacts employed as tools. The fluid interactions of people with one another, and with the situations they find themselves in, give rise to the continuous co-creation and transformation of those situations. Those transformations may be phenomenally perceived by internal or external observers and consequently judged as beneficial or not beneficial to the purposes of the organisation. It is here that the folk-psychological conception of leadership may be applied to a situation in order to judge the value of organisational change (but not its causal efficacy). The propositional attitudes of value, purpose and belief come into play when such judgements are made.

As Spillane (2006) discusses, the multiplicity of leaders within an organisation gives rise to a disjointed portrayal of leadership. If the relationship between a school principal and leading teacher is portrayed as a leader-follower relation how are the relations between principal and other teachers to be compared to the relations between the leading teacher and the other teachers? Spillane (2006, p. 21) suggests that 'the interrelations between teacher leadership and administrator leadership are rarely discussed'. By taking the focus of the actions of individuals and placing it on leadership practice as it occurs in communities of practice, the notion of distributed leadership enables the conjoint actions of teachers as leaders, and administrators as leaders, to be examined simultaneously within a given situation.

Distributed leadership is often portrayed in a functional sense, as something one does rather than in a conceptual sense, as description of the way things are (Bennett et al., 2003; Harris and Muijs, 2005, pp. 27-36). Confusion of terminology also arises with distributed leadership, which is falsely equated with collaborative leadership, shared leadership, co-leadership, democratic leadership, and situational leadership. In their review of distributed leadership in the literature, Bennett et al. (2003) identified considerable variation in the definitions of distributed leadership. Upon further analysis, they identified some salient features in the concept, describing it as a property that emerges from the interactions of individuals so that all people may 
potentially participate in leadership and contribute to the wide distribution and reach of expertise.

\subsection{Conclusion}

My thesis concerns a single two-dimensional account of organisational experience in schools: The causal-computational nature of organisations and the phenomenal essence of leadership. The former may provide a universal account of organisational functioning, whereas the latter is more suited to describing the qualities of first-person experience. Since both versions involve the concept of information, and both invoke the concept of emotion, there is potential for the two versions to interact. The result may be described as Distributed Cognition. I now seek to relate this to the questions posed in Chapter 3.

\subsubsection{To what extent are organisations the outworking of human cognition as described by theories of distributed cognition?}

In terms of distributed cognition theory, organisations arise entirely from the processes of human cognition. Just as the human brain comprises intricate, interconnected networks of cells, organisations comprise the interdependent actions of people, scaffolded by artefacts and environment. People interact with artefacts such as buildings, tools and technological devices, and the natural and social features of the environments in which they act. The interactions of brain, body and world, cannot be separated from the processes of distributed cognition; hence, to obtain better organisation theories requires that we improve our understanding of the distributed nature of human cognition. The natural sciences offer our best hope for understanding human cognition as the outworking of causal processes (in the sense of Aristotle's efficient cause). Therefore, cognitive science should be given a central place in the development of a generalised account of organisational functioning. The organisation and improvement of schools can be reasonably equated with the situated learning of their organisational members. Distributed cognition theory provides a means for explaining such learning in general, causal-computational terms. 


\subsubsection{To what extent can the functioning of schools as educational organisations be explained in terms of leadership?}

It is argued in this thesis that leadership is a quality or essence of phenomenology, not a causal concept of natural science. Leadership may form an important part of narrative accounts of organisational life, including the expressed intentions and beliefs of the participants of organisational life. However, a general, causal-computational account of organisational functioning cannot be legitimately constructed in terms of the phenomenal qualities of leadership. This is not to say that organisational members cannot express their personal experiences in the organisation in terms of leadership. Nor is it to say that researchers may not attempt to construct their own first-person account of leadership, as they perceive it in particular settings. However, it is argued in this dissertation that the description of organisational functioning, as the outworking of causal-computational processes, cannot be described legitimately as leadership. Nevertheless, whilst a generalised account of organisational functioning may be described in causal terms, the quality of an organisation - what it means to the people that work within it, or utilise its services may be described according to the phenomenological essence of leadership.

\subsubsection{Given the answers to the previous questions, what can a theory of distributed leadership offer?}

My thesis is that school functioning is best evaluated according to distributed cognition theory. Distributed cognition can offer a causal-computational account of the functioning of organisations in a way that acknowledges the interaction of people, artefacts and environment. It can also account for the learning of individuals and organisations. Whilst the concept of leadership may feature in explanations, phrased in terms of beliefs, purposes and hopes, its meaning will vary with the situations in which it is applied. Leadership itself can have no direct causalcomputational impact on organisations. However, phenomenological perceptions of leadership may interact with causal-computational accounts through the concepts of information and emotion. An organisational event may be associated with an emotional response in subjects, perhaps because organisational members believe leadership is a significant part of their experience. Under such circumstances, any 
belief or intention associated with an emotional response will also be represented within a distributed cognitive system, as described naturalistically.

The challenge for future research into school organisation and improvement, is to separate the causal-computational components of school life from the phenomenological experiences of being or becoming. This may be done by, firstly adopting research methods that enable interactions between people, artefacts and environment to be observed in accordance with our best theories of natural science; secondly, by identifying the various representations of information in those interactions; and thirdly, by identifying the serial and parallel-distributed computations through which information is processed. Analyses of this sort may contribute to the development of fruitful theoretical models of school functioning that may be successfully applied to a variety of situations.

Situational narratives of leadership may be constructed from the impressions, hopes and plans of those in the organisation and placed alongside causalcomputational analyses. Situational narratives may not legitimately refute a properly constructed causal-computational model, but may help participants in the organisation to draw meaning from their experiences in organisational life. Hence, the situational narrative of leadership may be placed alongside the causalcomputational account of distributed cognition, with such a dual account being known as distributed leadership. 


\section{References}

Achtenberg, D. (2002). Cognition of Value in Aristotle's Ethics. New York: State University of New York Press.

Adams, J. (1986). The Conspiracy of the Text: The Place of Narrative in the Development of Thought. London: Routledge and Kegan Paul.

Adler, J. (2006). Epistemological Problems of Testimony. In E. N. Zalta (ed.), The Stanford Encyclopedia of Philosophy (Winter 2006 Edition). Retrieved on 13 January 2007 from the website of Stanford Encyclopedia of Philosophy at http://plato.stanford.edu/archives/win2006/entries/testimony-episprob/.

African National Congress. (Circa 2004). Nelson Mandela. Retrieved on November 27, 2005 from The African National Congress website: http://www.anc.org.za/people/mandela.html

Allen, P. (1996). Who Sank the Boat? Melbourne: Thomas-Nelson Australia.

Allix, N. and Gronn, P. (2005). 'Leadership' as a Manifestation of Knowledge. In Educational Management Administration \& Leadership, Vol. 33, No. 2, pp. 181-196.

Allix, N. M. (2003, April). Epistemology and knowledge management concepts and practices. In Journal of Knowledge Management Practice, 4, April. Retrieved on December 13, 2006 from website of the Journal of Knowledge Management Practice: http://www.tlainc.com/articl49.htm

Alvesson, M. and Sveningsson, S. (2003). The great disappearing act: Difficulties in doing "leadership". In Leadership Quarterly, Vol. 14, No. 3, pp. 359-381.

Ambrose, S. E. (1991). Band of Brothers. New York: Simon and Schuster.

Anderson, C. J. (2004). Dick Winters: Reflections on the Band of Brothers, D-Day and Leadership. Originally published in American History (August, 2004). Retrieved on 9 December 2006 from the website of History Net at http://www.historynet.com/magazines/american_history/3029766.html?showA $\underline{1 l=y \& c=y}$. 
Anderson, F. (1975). The public school system of New South Wales. In A.G. Austin and R.J.W. Selleck. The Australian Government School 1830-1914 (pp. 295296). Extract reprinted from The Public School System of New South Wales, 1901, pp. 13-15. Carlton: Pitman.

Angeli, C. (2008) Distributed Cognition: A Framework for Understanding the Role of Computers in Classroom Teaching and Learning. In Journal of Research on Technology in Education, Vol. 40. No. 3, pp. 271-279.

Antonakis, J., Angerfelt, M., \& Sivasubramaniam, N. (2005). When she was good she was very good indeed but when she was bad she was horrid! Biasing effects on ratings of leadership. Women as Global Leaders Conference, Zayed University, Dubai, UAE.

Argyris, C. (1961). Organisational leadership. In L. Petrullo and B. M. Bass, Leadership and Interpersonal Behaviour (pp. 326-354). New York: Holt, Rinehart and Winston.

Argyris, C. and Schön, D. A. (1984). Organisational learning. In Pugh, D. S., Organisation Theory. Harmondsworth, Middlesex: Penguin.

Argyris, C. and Schön, D. A. (1996). Organisational Learning II: Theory, Method, and Practice. Reading, Massachusetts: Addison-Wesley.

Aristotle (1985). The Nicomachean Ethics. Translated by T. Irwin. Indianapolis, Indiana: Hackett.

Aristotle (1986). De Anima. Translated by Hugh Lawson-Tancred, London: Penguin [With introduction and notes by Hugh Lawson-Tancred, pp. 9-117, 222-251].

Aristotle (1994a). The Metaphysics. Retrieved on 2 August 2007 from the website of the Massachusetts Institute of Technology at http://classics.mit.edu/Aristotle/metaphysics.html.

Aristotle (1994b). The Physics. Retrieved on 2 August 2007 from the website of the Massachusetts Institute of Technology at http://classics.mit.edu/Aristotle/physics.html.

Aristotle (1994c). The Posterior Analytics. Retrieved on 2 August 2007 from the website of the Massachusetts Institute of Technology at http://classics.mit.edu/Aristotle/posterior.html. 
Aristotle (1995). The Nicomachean Ethics. (From a translation by W. D. Ross, 1908. Oxford: Clarendon Press). New York: Institute for Learning Technologies. Retrieved on 17 December 2006 from the website of Columbia University at http://www.ilt.columbia.edu/publicATIONS/artistotle.html.

Aristotle (2000). The Nicomachean Ethics. Trans. Roger Crisp (ed.), Cambridge: Cambridge University Press.

Arrowsmith, T. (2004). Distributed leadership: three questions, two answers - A review of the Hay Group Education research, July 2004. in Management in Education, Vol. 19, No. 2, pp. 30-33.

Australian Broadcasting Commission. (2002). Australians: Caroline Chisholm (1808-1887). Retrieved on November 27, 2005 from the Australian Broadcasting Commission website: http://www.abc.net.au/btn/australians/chisholm.htm

Axworthy, D. (2006, March). Improving learning: How turning data into information improves learning. In Professional Educator, Vol. 5, No. 1, pp. 12-13.

Ayer, A. J. (1935). The criterion of truth, In Analysis, 3, pp. 28-32. Reprinted in Paul Horwich (ed.), 1994, Theories of Truth. Aldershot, Hants, England: Dartmouth, pp. 101-106.

Bach, K. (2000). Do belief reports report beliefs? In K. M. Jaszczolt, The Pragmatics of Propositional Attitude Reports (pp. 111-136). Oxford: Elsevier.

Bacon, F. (1620). Novum Organum. Retrieved on 7 July 2007 from the website of the Constitution Society at http://www.constitution.org/bacon/nov_org.htm

Baker, M. (2004). Burma's junta ready to free Aung San Suu Kyi. In The Age (5 April, p. 9), Melbourne.

Balaguer, M. (2004). A Coherent, Naturalistic, and Plausible Formulation of Libertarian Free Will. In Noûs, Vol. 38, No.3, pp. 379-406.

Barry, D. (1991). Managing the bossless team: lessons in distributed leadership. In Organisational Dynamics, Vol. 20, No. 1, pp. 31-17.

Bass, B. M. (1985). Leadership and Performance Beyond Expectations. New York: The Free Press.

Bass, B.M. and Avolio, B.J. (1994). Introduction. In B.M. Bass, and B.J. Avolio, Improving Organisational Effectiveness Through Transformational Leadership (pp. 1-9). Thousand Oaks, California: Sage. 
Bates, R. (1991). The Management of Culture and Knowledge. Deakin University, Geelong, Victoria.

Baudrillard, J. (1988). Simulcra and Simulations. In M. Poster (ed.), Jean Baudrillard, Selected Writings, pp.166-184.Stanford; Stanford University Press.

Bayne, T. (2006). Phenomenology and the Feeling of Doing: Wegner on the Conscious Will. In S. Pockett, W. P. Banks \& S. Gallagher (eds.), Does Consciousness Cause Behaviour? An Investigation of the Nature of Volition (pp. 169-186). Cambridge, Massachusetts: MIT Press. Retrieved on 4 July 2007 from the website of Macquarie University at http://www.phil.mq.edu.au/staff/tbayne/Wegner.pdf

Bechara, A., Damasio, H., Tranel, D., and Damasio, A.R. (2005). The Iowa Gambling Task and the somatic marker hypothesis: some questions and answers. In Trends in Cognitive Sciences, Vol.9, No.4 (pp. 159-162). Retrieved on 25 January 2007 from the website of the Centre for the Neural Basis of Cognition, University of Pittsburgh at http://www.cnbc.cmu.edu/ jlm/papers/BecharaEtA105_TiCS.pdf.

Bennett, C. H. (1988). Logical Depth and Physical Complexity. In R. Herken, The Universal Turing Machine: a Half-Century Survey (pp. 227-257). New York: Oxford University Press. Retrieved on 27 January 2007 from the website of IBM at http://www.research.ibm.com/people/b/bennetc/UTMX.pdf.

Bennett, N., Wise, C., Woods, P. and Harvey, J. A. (2003). Distributed Leadership: A review of literature carried out for NCSL. London: National College for School Leadership. Retrieved on 24 January 2007 from the website of the National College for School Leadership at http://www.ncsl.org.uk/media/3C4/A2/distributed-leadership-literaturereview.pdf.

Bennis, W. G. (1959). Leadership Theory and Administrative Behaviour: The Problem of Authority. In Administrative Science Quarterly, Vol. 4, No. 3, pp. 259-301.

Bergman, G. (1954). Sense and Nonsense in Operationism. In The Scientific Monthly, Vol. 79, No. 4, pp. 210-214.

Berson, Y., Nemanich, L. A., Waldman, D. A., Galvin, B. M., and Keller, R. T. (2006). Leadership and organisational learning: A multiple levels perspective. In The Leadership Quarterly, Vol.17, pp. 577-594. 
Bertalanffy, L. von (1971). General System Theory: Foundations, Development, Applications. Harmondsworth, Middlesex, England: Penguin.

Blueprint for Government Schools (2003). Melbourne, Victoria: Department of Education. Retrieved on 2 August 2007 from the website of the Victorian Department of Education at http://www.sofweb.vic.edu.au/blueprint/.

Bohr, N. (1933). Light and life. In Nature, Vol. 131, pp. 421-423, 457-459.

Boje, D. M. and Rhodes, C. (2005). The virtual leader construct: The mass mediatisation and simulation of transformational leadership. In Leadership, Vol. 1, No. 4, pp. 407-428.

BonJour, L. (1985). The Structure of Empirical Knowledge. Cambridge, Massachusetts: Harvard University Press.

BonJour, L. (1988). In Defense of Pure Reason. London: Cambridge University Press.

BonJour, L. (1999), The dialectic of foundationalism and coherentism, in John Greco and Ernest Sosa, The Blackwell Guide to Epistemology, Blackwell, Massachusetts, pp. 117-142.

Bowe, R and Ball, S.J. (1992). Reforming Education and Changing Schools: Case Studies in Policy Sociology. London: Routledge.

Boyd, R. (2002). Scientific Realism. In E. N. Zalta (ed.), The Stanford Encyclopedia of Philosophy (Summer 2002 Edition). Retrieved on 7 July 2007 from the website of The Stanford Encyclopedia of Philosophy at http://plato.stanford.edu/archives/sum2002/entries/scientific-realism/

Bresnen, M. J. (1995). All things to all people? Perceptions, attributions, and constructions of leadership. In Leadership Quarterly, Vol. 6, No. 4, pp. 495513.

Broderick, D. (1994). The Architecture of Babel: Discourses of Literature and Science. Melbourne: Melbourne University Press.

Brooker, B. M. (2006). Stakeholders' Meanings of Effective School Leadership: A Case Study in a New Zealand Primary School. Dissertation submitted in partial fulfilment of Doctor of Education. School of Cognition, Language and Special Education. Faculty of Education. Griffith University. Retrieved on 1 January 2007 from the website of the Australian Digital Theses Program, Griffith University Site at http://www4.gu.edu.au:8080/adt-root/public/adtQGU20061023.151530/index.html. 
Brown, H. I. (1977). Perception and theory. In Perception, Theory and Commitment. Chicago: Precedent Publishing Inc.

Brown, M. H. (1989). Organising activity in the women's movement: An example of distributed leadership. In B. Klandermans (Ed.), International Social Movement Research, Vol. 2 (pp. 225-240). Greenwich, Connecticut: J. A. I. Press.

Brown, M. H. and Hosking, D. -M. (1986). Distributed Leadership and skilled performance as successful organisation in social movements. In Human Relations, Vol. 39, No. 1, pp. 65-79.

Bruner, J. (1986). Actual Minds, Possible Worlds. Cambridge, Massachusetts: Harvard University Press.

Bruner, J. (1990). Acts of Meaning. Cambridge Massachusetts: Harvard University Press.

Bryman, A. (1996). Leadership in organisations. In S. R. Clegg, C. Hardy and W. R. Nord (Eds.), Handbook of Organisation Studies (pp. 276-292). London: Sage.

Bullock, A., Stallybrass, O. and Trombley, S. (eds.) (1988). The Fontana Dictionary of Modern Thought, 2nd ed. London: Fontana Press.

Bunge, M. (1983), Treatise on Basic Philosophy, Volume 6: Epistemology and Methodology II - Understanding the World. Dordrecht Holland: D. Reidel Publishing Company,.

Burnett, F. H. (1956). The Secret Garden. London: The Reprint Society.

Burns, J.M. (1978). Leadership. New York: Harper \& Row.

Byrne, M. N. (2003). Mustafa Kemal Ataturk - Commander and an Effective Leader, In Geddes Papers. Canberra: Australian Defence College. Retrieved on November 27, 2005 from the website of the Australian Government Department of Defence: http://www.defence.gov.au/adc/docs/Publications/Geddes\%20Papers\%202003/ 04\%20Mustafa\%20Kemal_Byrne.pdf

Cacace, F. (1997). Meaning, reality and algorithms: implications of the Turing theorem. In A. Driessen and A. Suarez (eds.), Mathematical Undecideability, Quantum Nonlocality and the Question of the Existence of God (pp. 27-40). Dordrecht, The Netherlands: Kluwer Academic Publishers. 
Caldwell, B. and Spinks, J. (1991). Policy Formation and Resource Allocation. Geelong, Victoria: Deakin University.

Caldwell, B.J. and Hayward, D.K. (1998). The Future of Schools: Lessons from the Reform of Public Education. London: Falmer Press.

Caldwell, B.J. and Spinks, J.M. (1988). The Self-Managing School. Lewes: Falmer Press.

Caldwell, B.J. and Spinks, J.M. (1992). Leading the Self-Managing School. Lewes: Falmer Press.

Callahan, R. E. (1962). Education and the Cult of Efficiency: A Study of the Social Forces That Have Shaped the Administration of the Public Schools. Chicago: University of Chicago Press.

Callinicos, A. (1995), Theories and Narratives: Reflections on the Philosophy of History. Duke University Press, Durham.

Campbell, D. P. (1991). Campbell Leadership Index Manual. Minneapolis: National Computer Systems.

Campion, T. (1900). Devotion (i). In Arthur Quiller-Couch (Ed.), The Oxford Book of English, Verse (pp. 204-205). Oxford: Clarendon Press.

Canedy, D. (2007). Love after death: A soldier's journal for his son. In The Age (2 January, News, pp. 1, 9).

Carlyon, L. (2001). Gallipoli. Sydney: Macmillan.

Carlyon, L. (2006). The Great War. Sydney: Macmillan.

Carroll, L. (1984). Alice's Adventures in Wonderland. Harmondsworth: Puffin.

Carroll, N. (1999). Film, emotion and genre. In C. Plantinga and G. M. Smith, Passionate Views: Film, Cognition and Emotion (pp. 21-47). Baltimore: The Johns Hopkins University Press.

Carter, J.A. (2003). Telling Times: History, Emplotment, and Truth. In History and Theory; Vol. 42 Issue 1, pp1-27.

Carter, R. (1999). Mapping the Mind. London: Orion. 
Cauchi, S. (2003a). Bible Doubter's Theory Down the Drain. In The Age, 11 September, page number not known.

Cauchi, S. (2003b). Leskie Bib Puts Science in the Dock. In The Age, 22 November, Insight, p. 9.

Cauchi, S. (2004). Lost Race of Human 'Hobbits' Unearthed on Indonesian Island. In The Age, 28 October, pp. 1, 2.

Chaitin G. J. (1988). An algebraic equation for the halting probability. In R. Herken, The Universal Turing Machine (pp. 279-283). New York: Oxford University Press. Retrieved on 16 February 2007 from the website of the University of Auckland at http://www.cs.auckland.ac.nz/CDMTCS/chaitin/berlin.pdf.

Chaitin, G. J. (1969). On the Simplicity and Speed of Programs for Computing Infinite Sets of Natural Numbers. In Journal of the ACM, Vol. 16, No. 3, pp. 407-422.

Chaitin, G. J. (1995). A new version of algorithmic information theory. In Complexity, Vol. 1, No. 4, pp. 55-59.

Chaitin, G. J. (1997). Number and randomness: algorithmic information theory new results on the foundation of mathematics. In A. Driessen and A. Suarez (eds.), Mathematical Undecideability, Quantum Nonlocality and the Question of the Existence of God (pp. 15-25). Dordrecht, The Netherlands: Kluwer Academic Publishers.

Chaitin, G. J. (1999). The Unknowable. New York: Springer-Verlag.

Chaitin, G. J. (2006). How real are real numbers? In International Journal of Bifurcation and Chaos, Vol. 16, No. 6, pp. 1841-1848.

Chaitin, G.J. (1996). The limits of mathematics (pp. 270-305). In Journal of Universal Computer Science, 2, No. 5. Retrieved on 25 November 2006 from the website of the University of Auckland, Department of Computer Science at http://www.cs.auckland.ac.nz/CDMTCS/chaitin/rov.html.

Chalmers, D. J. (1994). Perception and the fall from Eden. In T. Gendler and J. Hawthorne (Eds.), Perceptual Experience (pp. 49-125). Oxford: Oxford University Press.

Chalmers, D. J. (1995). Minds, machines, and mathematics: a review of shadows of the mind by Roger Penrose. In Psyche, Vol. 2, No. 9. Retrieved on 16 February 2007 from the website of $P$ syche at http://psyche.cs.monash.edu.au/v2/psyche2-09-chalmers.html. 
Chalmers, D. J. (1996). The Conscious Mind: In Search of a Fundamental Theory. New York: Oxford University Press.

Chalmers, D. J. (1998). On the Search for the Neural Correlate of Consciousness. In S. Hameroff, A. Kaszniak, \& A. Scott (eds.). Toward a Science of Consciousness II (pp. 219-230). Cambridge, Massachusetts: MIT Press. Retrieved on 24 July 2007 from the website of David Chalmers at http://consc.net/papers/ncc.pdf.

Chalmers, D. J. (2002). On sense and intension. In J. Tomberlin (ed.), Philosophical Perspectives, Vol. 16: Language and Mind (pp. 135-182). Blackwell. Retrieved on 19 July 2007 from the website of David Chalmers at http://consc.net/papers/intension.html.

Chalmers, D. J. (2006). Strong and Weak Emergence. In P. Clayton and P. Davies (eds.), The Re-Emergence of Emergence (pp. 244-256). Oxford: Oxford University Press. Retrieved on 24 July 2007 from the website of David Chalmers at http://consc.net/papers/emergence.pdf.

Chandler, A.D. Jr (1969). Strategy and Structure: Chapters in the History of the American Industrial Enterprise (Paperback edition). Massachusetts: MIT Press.

Chandler, J. (2005, December 27). Stem Cell Fraud Could Harm Research, Say Experts. In The Age, 27 December, p. 3.

Chevalier, T. (2000). Girl With a Pearl Earring. New York: Dutton.

Chomsky, N. (1957). Syntactic Structures. The Hague, Netherlands: Mouton.

Churchland P.S. and Sejnowski, T.J. (1992). The Computational Brain. Cambridge, Massachusetts: Bradford.

Churchland, P.M. (1981). Eliminative Materialism and the Propositional Attitudes, Journal of Philosophy, Vol. 78, pp. 67-90.

Churchland, P.M. (1995), The Engine of Reason, the Seat of the Soul. Cambridge Massachusetts: MIT Press.

Churchland, P.S. (1986). Neurophilosophy: Toward a Unified Science of the Mind/Brain. Cambridge, Massachusetts: MIT Press.

Clancy, T. (1993). Red Storm Rising. London: Harper Collins. 
Clandinin, D. J. and Connelly, F. M. (2000). Narrative Inquiry: Experience and Story in Qualitative Research. San Francisco, California: Jossey-Bass.

Clare, G. (1990). Berlin Days: 1946-47. London: Pan Books.

Clark, A. (1989). Microcognition: Philosophy, Cognitive Science, and Parallel Distributed Processing. Cambridge, Massachusetts: The MIT Press.

Clark, A. (1997), Being There: Putting Brain, Body and World Back Together Again. Cambridge, Massachusetts: Bradford.

Clark, A. and Chalmers, D.J. (2002). The extended mind. In D.J. Chalmers (ed.), Philosophy of Mind: Classical and Contemporary Readings (pp. 543-652). New York: Oxford University Press.

Clarke, D.J. (2004). Patterns of Participation in the Mathematics Classroom. In M.J. Høines \& A.B. Fuglestad (Eds.), Proceedings of the 28th Conference of the International Group for the Psychology of Mathematics Education, Vol. 2, (pp. 231-238). Bergen: Bergen University College.

Clements, C. and Washbush, J. B. (1999). The two faces of leadership: considering the dark side of leader-follower dynamics. In Journal of Workplace Learning, Vol.11, No. 5, pp. 170-176.

Cohen, M. C. (2006). The Four Causes. Retrieved on 2 August 2007 from the website of the University of Washington at http://faculty.washington.edu/smcohen/320/4causes.htm

Cohen, S. M. (2003). Aristotle's Metaphysics. In E. N. Zalta (ed.), The Stanford Encyclopedia of Philosophy (Summer 2003 Edition). Retrieved on 2 August 2007 from the website of The Stanford Encyclopedia of Philosophy at http://plato.stanford.edu/archives/sum2003/entries/aristotle-metaphysics/.

Collins, M. A. (1998). Saving Private Ryan. London: Penguin.

Colston, H. L. (2005). On sociocultural and nonliteral: A synopsis and a prophecy. In H. L. Colston and A. N. Katz, Figurative Language Comprehension: Social and Cultural Influences (pp. 1-18). Mahwah, New Jersey: Lawrence Erlbaum Associates.

Connolly, P. (2006). The Masculine Habitus as 'Distributed Cognition': A Case Study of 5- to 6-Year-Old Boys in an English Inner-City, Multi-Ethnic Primary School. In Children and Society, Vol. 20. No. 2, pp. 140-152. 
Cook, J. (2006, March). Reporting to systems and schools. In Professional Educator, Vol. 5, No. 1, pp. 6-10.

Cooperative Research Project (1998). Report of the Cooperative Research Project on "Leading Victoria's Schools of the Future". Victorian Education Department, Victorian Association of State Secondary Principals, Victorian Primary Principals Association, The University of Melbourne.

Cresswell, M. J. (1985). Structured Meanings: The Semantics of the Propositional Attitudes. Cambridge, Massachusetts: MIT Press.

Cringley, R. X. (1996). Accidental Empires. London: Penguin.

Damasio, A. R. (1994). Descartes' Error: Emotion, Reason and the Human Brain. New York: G.P. Putnam Sons.

Damasio, A. R. (2000). The Feeling of What Happens: Body, Emotion and the Making of Consciousness. London: Vintage.

Davies, A. (1998). Thinking about 'narrative reasoning' as a methodology. In Curriculum Inquiry, Vol. 28, No. 4, pp. 473-483.

Davies, M. and Stone, T. (eds.) (1995) Folk Psychology: The Theory of Mind Debate. Oxford: Blackwell.

Davies, P. (1992). The Mind of God: Science and the Search for Ultimate Meaning. London: Penguin Books.

Davies, P. (2005). About Time: Einstein's Unfinished Revolution. London: Penguin.

Davies, P. (2006). The Goldilocks Dilemma: Why Is the Universe Just Right for Life? London: Allen Lane.

De Bono, E. (1976). Practical Thinking. London: Penguin

De Preester, H. (2006). Naturalism and transcendentalism in the naturalisation of phenomenology. In New Ideas in Psychology, Vol. 24, pp. 41-62.

De Sousa, R. (1980). The rationality of emotions. In A. Rorty (ed.), Explaining Emotions (pp. 127-152). Berkeley: University of California Press.

Dennett, D. (1991). Consciousness Explained, Boston: Little, Brown. 
Denning, S. (2005). The Leader's Guide to Storytelling: Mastering the Art and Discipline of Business Narrative. San Francisco, CA: Jossey-Bass.

Denzin, N.K. (1988). Triangulation. In J.P. Keeves (ed.), Educational Research Methodology and Research: An International Handbook (pp. 511-513). Oxford: Pergamon.

Developmental Learning Framework for School Leaders (2007). Melbourne, Victoria: Department of Education.

Dobbs, D. (2006). A Revealing Reflection. In Scientific American Mind. April, 2006. Retrieved from the website of David Dobbs on 8 January 2007 at http://daviddobbs.net/page2/page4/mirrorneurons.html.

Donmoyer, R. (2001). Evers and Lakomski's search for leadership's Holy Grail (and the intriguing ideas they encountered along the way). In Journal of Educational Administration, 39 (6), 554-572. Retrieved December 23, 2005 from the Emerald website:

http://www.emeraldinsight.com/Insight/viewPDF.jsp?Filename=html/Output/P ublished/EmeraldFullTextArticle/Pdf/0740390604.pdf

Eddy, P. L. (2005). Framing the Role of Leader: How Community College Presidents Construct Their Leadership. In Community College Journal of Research \& Practice, Vol. 29, No. 9/10, pp. 705-727.

Eden, D., and Leviatan, U. (1975). Implicit leadership theory as a determinant of the factor structure underlying supervisory behavior scales. In Journal of Applied Psychology, Vol. 60, pp. 736-741.

Edmondson, W. H. and Beale, R. (2008). Projected Cognition - extending Distributed Cognition for the study of human interaction with computers. In Interacting with Computers, Vol. 20, No. 1, pp. 128-140.

Education: the Defects of the Common School System (1871). Melbourne, pp. 4-7. Reprinted in A.G. Austin and R.J.W. Selleck, (1975), The Australian Government School 1830-1914 (pp. 42-45). Carlton: Pitman.

Edwards, D. (1997). Discourse and Cognition. London: Sage Publications.

Ehrich, L. C. (2005). Revisiting phenomenology: its potential for management research. In Proceedings Challenges or organisations in global markets, British Academy of Management Conference (pp. 1-13). Oxford: Said Business School, Oxford University. Retrieved on 7 July 2007 from the website of Queensland University of Technology at http://eprints.qut.edu.au/archive/00002893/01/2893.pdf 
Ellis, G. F. R. (2003). On the nature of emergent reality. Unpublished paper.

Retrieved on 11 June 2007 from the website of the Department of Mathematics and Applied Mathematics, University of Capetown at http://www.mth.uct.ac.za/ ellis/emerge

Ellis, G.F.R. (2005). Physics, Complexity and Causality. In Nature, Vol. 435, p. 743.

End User (2007). Problem in chair, not in computer. In APC Magazine, January, pp.28-29.

English, F. W. (2002). The Point of Scientificity, the Fall of the Epistemological Dominos, and the End of the Field of Educational Administration. In Studies in Philosophy \& Education, Vol. 21, No. 2, pp. 109-136.

Erneling, C. E. and Johnson, D. M. (2005). The Mind as a Scientific Object: Between Brain and Culture. Oxford: Oxford University Press.

Evers, C. W. (2000a). Leading and learning in organizational contexts: a contribution from the new cognitive science. In International Journal of Leadership in Education, Vol. 3. No.3, pp. 239-254.

Evers, C. W. (2000b). Connectionist modelling and education. In The Australian Journal of Education, 44 (3), 209-225.

Evers, C. W. and Lakomski, G. (1991). Knowing Educational Administration: Contemporary Methodological Controversies in Educational Administration Research. Oxford: Pergamon Press.

Evers, C. W. and Lakomski, G. (1995). Towards coherence in administrative theory. In C. W. Evers and J. Chapman (eds.), Educational Administration: An Australian Perspective (pp. 97-109). St. Leonhards: Allen \& Unwin.

Evers, C. W. and Lakomski, G. (1996). Exploring Educational Administration: Coherentist Applications and Critical Debates. Oxford: Pergamon Press.

Evers, C. W. and Lakomski, G. (2001). Theory in educational administration: naturalistic directions. In Journal of Educational Administration, Vol. 39, No. 6, pp. 499-520.

Evers, C.W. and Lakomski, G. (2000). Doing Educational Administration: A Theory of Administrative Practice. Oxford: Pergamon Press. 
Expression of Interest for Master in School Leadership, and if applicable, Young Leaders Program, through the Blueprint Initiative: Accelerated Development for High Potential Leaders (2004a). Melbourne, Victoria: Department of Education and Training, April 26.

Fell, L. (1999), Songlines in the City? Hearing the spirit dimension, from the proceedings of the $2^{\text {nd }}$ Conference on Spirituality, Leadership and Management. Downloaded on 2 March 2004 from the website of the Proceedings of the 2nd Conference on Spirituality, Leadership and Management http://www.pnc.com.au/ lfell/songline.html.

Feyerabend, Paul K. (1976). The rationality of science, in Sandra G. Harding, Can Theories be Refuted? (pp. 289-315).Boston: Reidal.

Fiedler, F. E. (1967). A Theory of Leadership Effectiveness, Vol. 1. New York: Academic Press.

Fiedler, F. E. (1972). The effects of leadership training and experience: A contingency model interpretation. In Administrative Science Quarterly, Vol 17, No. 4, pp. 453-470.

Fiedler, F. E. (1978). The contingency model and the dynamics of the leadership process. In Leonard Berkowitz (Ed.), Advances in Experimental Social Psychology. Vol. 12, (pp. 59-112). New York: Academic Press.

Fiedler, F. E. and Garcia, J. E. (1987). New approaches to effective leadership: Cognitive resources and organizational performance. New York: John Wiley \& Sons.

Field, H. (1994). Tarski's theory of truth (originally published in Journal of Philosophy, 1972, 69. pp. 347-375). In Paul Horwich (ed.), Theories of Truth (pp. 285-314). Aldershot, Hants: Dartmouth,

Foster, W. (1986). The Reconstruction of Leadership. Geelong: Deakin University.

Frumkin, A., A. Shimron, and J. Rosenbaum. (2003). Radiometric dating of the Siloam Tunnel, Jerusalem. In Nature, No. 425, pp. 169-171.

Fullan, M. (1993). Change Forces: Probing the Depths of Educational Reform. London: Falmer Press.

Fullan, M. (2005). Leadership Sustainability. Thousand Oaks, California: Corwin Press. 
Fyfe, M. (2004). Point Nepean plan in chaos as groups quit. In The Age (25 March). Retrieved on 11 July 2007 from the website of The Age at http://www.theage.com.au/articles/2004/03/24/1079939722355.html

Gaader, J. (1996). Sophie’s World. London: Phoenix.

Galison, Peter (2003), Einstein's Clocks, Poincaré's Maps: Empires of Time. London: Hodder and Stoughton.

Gallese, V. (2005). Embodied simulation: From neurons to phenomenal experience. In Phenomenology and the Cognitive Sciences, Vol. 4, pp. 23-48. Retrieved on 31 January 2007 from the website of the Universita Degli Studi Di Parma at http://www2.unipr.it/ gallese/PCS2005.pdf.

Gardner, H. (1983). Frames of Mind: The theory of multiple intelligences,. New York: Basic Books.

Gartner, A., Latham, G. and Merritt, S. (1996). The power of narrative: transcending disciplines. In UltiBASE. Melbourne: RMIT University. Retrieved on 4 December 2006 from the website of the RMIT University at http://ultibase.rmit.edu.au/Articles/dec96/gartn1.htm.

Gavriel, S. (1993). Distributed cognitions: Psychological and educational considerations. Cambridge: Cambridge University Press.

Gibb, C.A. (1954). Leadership. In G. Lindzey (Ed.), Handbook of Social Psychology, vol. 2 (pp. 877-917), Reading, MA: Addison-Wesley.

Gibb, C.A. (1969). Leadership. In G. Lindzey \& E. Aronson (Eds.), The Handbook of Social Psychology, vol. 4 (2nd. Ed.), Reading, MA: Addison-Wesley, pp. 205-283.

Giere, R. N. and Moffatt, B. (2003). Distributed cognition: Where the cognitive and the social merge. In Social Studies of Science, Vol. 33, No. 2., pp. 301-310.

Gijsbers, A. (2005). Letter. In ISCAST Bulletin, Issue 48, p. 6.

Gilbert, G. P. (2005). Re-evaluating the Battle of Trafalgar. In Australian Defence Force Journal, No. 168, pp. 7-18. Retrieved on 27 November 2005 from the website of the Australian Government Department of Defence at http://www.defence.gov.au/publications/dfj/adfj168.pdf

Girl Moved To Tears By “Of Mice And Men” Cliffs Notes (2006), 16 August. Retrieved on 4 January 2006 from the website of the parody newspaper, The Onion at http://www.theonion.com/content/node/52061. 
Gödel, K. (1995). The modern development of the foundations of mathematics in the light of philosophy (circa 1961). In S. Feferman, J.W. Dawson, Jr., W. Goldfarb, C. Parsons, and R.M. Solovay (eds.), Kurt Gödel: Collected Works, Vol. III., pp. 374-387. Oxford: Oxford University Press.

Goos, M. (2002), New technologies, new pedagogies: the mathematics classroom as a learning community, in C. Vale, J. Roumeliotis, \& J. Horwood (eds.), Valuing Mathematics in Society, Proceedings of the 39th Annual Conference of the Mathematics Association of Victoria (pp. 52-64). Brunswick: Mathematics Association of Victoria.

Gordon, R. M. (1995), Folk psychology as simulation. In M. Davies and T. Stone (eds.), Folk Psychology: Readings in Mind and Language (pp. 60-73). Oxford: Blackwell.

Graen, G. B. (2003). Role making onto the starting team using LMX leadership diversity as an asset. In G. B. Graen, Dealing With Diversity (pp. 1-28). Greenwich, Connecticut: Information Age Publishing.

Grant, S. G. N. (2003a). An integrative neuroscience program linking mouse genes to cognition and disease. In R. Plomin, J.C. Defries, I.W. Craig and P. McGuffin (eds.) Behavioural Genetics in the Post Genomic Era (pp. 123-138). Washington D.C: American Psychological Association.

Grant, S. G. N. (2003b). Synapse signalling complexes and networks: machines underlying cognition. In BioEssays, Vol. 25, No.12, pp. 1229-1235.

Greenfield, T. (1975). Theory about organisation: a new perspective and its implications for schools. In M. G. Hughes (ed.), Administering Education: International Challenge. London: Athlone Press.

Greenfield, T. (1986). The decline and fall of science in educational administration. In Interchange, Vol. 17, No. 2, pp. 57-80.

Greenfield, T. B. (1980). The man who comes back through the door in the wall: Discovering truth, discovering self, discovering organisations. In Educational Administration Quarterly, Vol.16, No.3, pp. 26-59.

Grob, L. (1984). Leadership: The Socratic model. In B. Kellerman (ed.), Leadership: Multidisciplinary Perspectives (pp. 263-280). Englewood Cliffs, New Jersey: Prentice-Hall.

Gronn, P. (2000). Distributed properties: a new architecture for leadership. In Educational Management and Administration, Vol. 28, No. 3., pp. 317-338. 
Gronn, P. (2002a). Distributed Leadership as a unit of analysis. In Leadership Quarterly, Vol. 13, pp. 423-451.

Gronn, P. (2002b). Distributed leadership. In K. Leithwood and P. Hallinger (Ed.). Second International Handbook of Educational Leadership and Administration (pp. 653-696). Dordrecht: Kluwer Academic Publishers.

Gronn, P. (2003). The New Work of Educational Leaders. London: Paul Chapman Publishing.

Gronn, P. (2006). The significance of distributed leadership. In BC Educational Research, No. 7. Retrieved on 2 March 2008 from the website of the University of British Colombia at http://slc.educ.ubc.ca/eJournal/Issue7/Articles/DistributedLeadership_\%20Pete r\%20Gronn.pdf

Grush, R. and Churchland, P. S. (1995). Gaps in Penrose's Toiling. In Journal of Consciousness Studies, Vol. 2, No. 1, pp. 10-29. Retrieved on 2 August 2007 from the website of the University of California, San Diego, at http://mind.ucsd.edu/papers/penrose/penrosehtml/penrose-text.html

Gudmundsdottir, S. (1991). Story-maker, story-teller: Narrative structures in curriculum. In Journal of Curriculum Studies, Vol. 23, No. 3, pp. 207-18.

Gwynne, P. (1998). Deadly Unna. Camberwell, Victoria: Penguin

Habermas, J. (1984). The Theory of Communicative Action (Vol. 1): Reason and the Rationalisation of Society. Boston, Massachusetts: Beacon Press.

Hall, J. (2005). Neuroscience and Education: A Review of the Contribution of Brain Science to Teaching and Learning. SCRE Research Report No. 121, Scottish Council for Research in Education. Glasgow: University of Glasgow.

Halverson, R. R. and Clifford, M. A... (2006). Evaluation in the Wild: A Distributed Cognition Perspective on Teacher Assessment. In Educational Administration Quarterly, Vol. 42, No. 4, pp. 578-619.

Hamilton, D. (1990). Learning about Education: An Unfinished Curriculum. Milton Keynes: Open University Press.

Harris, A. (2004). Teacher leadership and distributed leadership: An exploration of the literature. In Leading and Managing Vol. 10, No 2, pp. 1-10.

Harris, A. (2005a). Crossing boundaries and breaking barriers: Distributing leadership in schools. London: Specialist Schools Trust. 
Harris, A. (2005b). Leading or misleading? Distributed leadership and school improvement. In Journal of Curriculum Studies, Vol. 37, No.3, pp.255-267. Retrieved on 3 January 2006 from the website of the University of Illinois at Urbana Champaign: http://faculty.ed.uiuc.edu/westbury/JCS/Vol37/harris.htm

Harris, A. (2006). Opening up the Black Box of Leadership Practice: Taking a Distributed Perspective. In International Journal of Educational Administration, Vol. 34, No. 2, pp. 37-46.

Harris, A. and Muijs, D. (1995). Improving Schools Through Teacher Leadership. Maidenhead, Berkshire: Open University Press.

Hatcher, R. (2005). The distribution of leadership and power in schools. In British Journal of Sociology of Education, Vol. 26, No. 2, pp. 253-267.

Hawke, B. (1994). The Hawke Memoirs. Melbourne: William Heinemann.

Hawkes, T. (1977). Structuralism and Semiotics. Berkeley, California: University of California Press.

Hay Group Education (2004). The Five Pillars of Distributed Leadership in Schools: An Investigation Into the Advantages and Disadvantages, Causes and Constraints of a More Distributed Form of Leadership in Schools. Nottingham: National College of School Leadership.

Healey, R. (2004). Holism and nonseparability in physics. In E. N. Zalta (Ed.). The Stanford Encyclopaedia of Philosophy, Winter 2004 Edition. Retrieved January 3, 2006 from the website of the Stanford Encyclopaedia of Philosophy: http://plato.stanford.edu/archives/win2004/entries/physics-holism/.

Hebb, D.O (1949). Organisation of Behaviour. New York: Wiley

Heelas, P. (1996). Emotion talk across cultures. In R. Harré and W. G. Parrott (eds.). The Emotions: Social, Cultural and Biological Dimensions (pp. 171-199). London: SAGE.

Heidegger, M. (1962). Being and Time (translated by J. Macquarrie and E. Robinson). Originally published in German as Sein und Ziet, 1927. New York: Harper and Row.

Hempel, C. G. and Oppenheim, P. (1948). Studies in the logic of explanation. In Philosophy of Science, Vo. 15, No. 2, pp. 135-175

Heracleous, L. (2006). Discourse, Interpretation, Organisation. Cambridge: Cambridge University Press. 
Hersey, P. (1984) The situational leader. Escondido: The Center For Leadership Studies.

Hersey, P. and Blanchard, K. H. (1995). Behavioural theories of leadership. In J. Thomas Wren (ed.), Leader's Companion: Insights on Leadership Through the Ages (pp. 144-148). New York: The Free Press.

High Court of Australia (1989). Mabo and Another V. The State of Queensland and Another, 166 CLR 186 F.C. 88/062 . Retrieved on 2 August 2007 from the website of the Australasian Legal Information Institute at http://www.austlii.edu.au/au/cases/cth/high_ct/166clr186.html.

Hildreth, P. and Kimble, C. (2002). The Duality of Knowledge. In Information Research, Vol. 8, No. 1, Paper no. 142. Retrieved on 7 August 2007 from the website of Information Research at http://InformationR.net/ir/81/paper142.html]

Hill, A. L. (2007). Knowing Schools as Organisations: A Naturalistic Coherentist Theory of Knowledge. In Post-Script: Postgraduate Journal of Education Research, Vol. 8, No.1, pp.88-101. Retrieved on 16 March 2008 from the website of the University of Melbourne at http://www.edfac.unimelb.edu.au/research/resources/student_res/Andrew_Hill. pdf

Hill, W. (1969). The Validation and Extension of Fiedler's Theory of Leadership Effectiveness. In The Academy of Management Journal, Vol. 12, No. 1, pp. 3347.

Hill, W. F. (1981). Principles of Learning: A Handbook of Applications. Sherman Oaks, California: Alfred Publishing.

Hitler, A. (1943). Mein Kampf. Boston: Houghton Mifflin.

Hocutt, M. (1974). Aristotle's Four Becauses. In Philosophy, Vol. 49, No. 190, pp. 385-399.

Holden, S. (2004). Teachers Matter. In Professional Educator (Vol. 3, No. 1, pp. 2022).

Hollander, E. P. and Julian, J. W. (1969). Contemporary Trends in the Analysis of Leadership Processes. In Psychological Bulletin, Vol. 71, No. 5, pp. 387-91. 
Holtgraves, T. H. (2005). Social and cultural influences on figurative and indirect language. In H. L. Colston and A. N. Katz, Figurative Language Comprehension: Social and Cultural Influences (pp. 99-130). Mahwah, New Jersey: Lawrence Erlbaum Associates.

Horwich, P. (ed.) (1994). Theories of Truth. Aldershot, Hants, England: Dartmouth, pp. xi-xvii.

Hoy, W. K. and Miskel, C. G. (1987). Educational Administration: Theory, Research and Practice, $3^{\text {rd }}$ edition. New York: Random House.

Hughes, R. L., Ginnett, R. C. and Curphy, G. R. (1995), 'What is leadership', in J. Thomas Wren (ed.), Leader's Companion: Insights on Leadership Through the Ages, The Free Press, New York, pp. 39-43.

Hume, D. (1975). A Treatise of Human Nature, edited by L. A. Selby-Bigge, 2nd ed. revised by P.H. Nidditch. Oxford: Clarendon Press.

Hutcheson (1981). Solipsistic and intersubjective phenomenology. In Human Studies, Vol. 1, No. 4, pp. 185-178.

Hutchins, E. (1995). Cognition in the Wild. Cambridge, Massachusetts: MIT Press.

Hutchins, E. and Klausen, T. (1996). Distributed cognition in an airline cockpit. In Y. Engeström and D. Middleton (Eds.), Cognition and communication at work (pp. 15-34). New York: Cambridge University Press.

Hywood, G. (2004). An example to our leaders. The Age (1 ${ }^{\text {st }}$ April, p. 15). Melbourne. Retrieved on 11 July 2007 from the website of The Age at http://www.theage.com.au/articles/2004/03/31/1080544552673.html

Interview B5. Transcript of an interview conducted with the principal of 'Eucalypt Primary School', 14 March 1988.

Interview B6. Transcript of an interview conducted with the school council president of 'Eucalypt Primary School', 14 March 1988.

Interview B7. Transcript of an interview conducted with the assistant principal of 'Eucalypt Primary School', 14 March 1988.

Interview B8. Transcript of an interview conducted with a leading teacher of 'Eucalypt Primary School', 14 March 1988. 
Irvine, A. D. (June 2004). Russell's Paradox. In The Stanford Encyclopedia of Philosophy (Summer 2004 Edition), Edward N. Zalta (ed.), http://plato.stanford.edu/archives/sum2004/entries/russell-paradox/

Israel, D. J. (1987). The Role of Propositional Objects in Action. Stanford: Centre for the Study of Language and Information.

Jacob, P. (2003). Intentionality. In E. N. Zalta (ed.), The Stanford Encyclopedia of Philosophy (Fall 2003 Edition). Retrieved on 11 February 2007 from the website of The Stanford Encyclopedia of Philosophy at http://plato.stanford.edu/archives/fall2003/entries/intentionality/.

Jago, G. (1982). Leadership: Perspectives in theory and research. In Management Science, Vol. 28, No. 3, pp. 315-336.

Jakobson, R. (1956). Fundamentals of Language. Janua Linguarium, Series Minor, I. The Hague: Mouton.

Johnson, D. M. (2005). Can cognitive science locate and provide a correct account of the mind's centre? Progress towards the literal. In C. E. Erneling and D. M. Johnson, The Mind as a Scientific Object: Between Brain and Culture (pp. 314). Oxford: Oxford University Press.

Johnson, D.W. \& Johnson, R.T. (1989). Leading the Cooperative School. Minnesota: Interaction Book Company.

Kaldor, P., Bellamy, J. and Powell, R. (1997). Shaping a Future: Characteristics of Vital Congregations. Adelaide: Openbook.

Kane, R. (1999). Responsibility, Luck, and Chance: Reflections on Free Will and Indeterminism. The Journal of Philosophy, Vol. 96, No. 5, pp. 217-240.

Kant, I. (1964), Critique of Pure Reason, translation by Norman Kemp Smith. London: Macmillan,

Kant, I. (2005). Critique of Practical Reason. Liberty Fund. http://oll.libertyfund.org/EBooks/Kant_0431.pdf

Kaplan, A. (1999). Scientific methods in educational research. In J. P. Keeves and G. Lakomski, Issues in Educational Research (pp. 79-91). Oxford: Pergamon.

Karl, B. D. (1970). The power of intellect and the politics of ideas. In D. A. Rustow, Philosophers and Kings: Studies in Leadership (pp. 431-464). New York: George Braziller. 
Karmel, B. (1978). Leadership: A challenge to traditional research methods and assumptions. Academy of Management Review, Vol. 3, No. 3, pp. 475-482.

Katz, A. N. (2005a). Preface. In H. L. Colston and A. N. Katz, Figurative Language Comprehension: Social and Cultural Influences (pp. ix-xi). Mahwah, New Jersey: Lawrence Erlbaum Associates.

Katz, A. N. (2005b). Discourse and sociocultural factors in understanding nonliteral language. In H. L. Colston and A. N. Katz, Figurative Language Comprehension: Social and Cultural Influences (pp. 183-208). Mahwah, New Jersey: Lawrence Erlbaum Associates.

Katz, D. and Kahn, R. (1966). Organisations and the system concept. In The Social Psychology of Organisations (pp. 14-29). New York: John Wiley and Sons.

Keeves, J. P. (1999). Overview of Issues in Educational Research. In J. P. Keeves and G. Lakomski, Issues in Educational Research (pp. 3-14). Oxford: Pergamon.

Kelly, K. T. (2001). Learning theory and epistemology. Retrieved on 10 July 2006 from the website of Carnegie Mellon University at www.hss.cmu.edu/philosophy/kelly/papers/learnreview.pdf.

Kennedy, J. (2007). Kurt Gödel. In E. N. Zalta (ed.), The Stanford Encyclopedia of Philosophy. Retrieved on 11 March 2007 from the website of the Stanford Encyclopedia of Philosophy at http://plato.stanford.edu/entries/goedel/. Forthcoming archive of the Spring 2007 Edition to be created at http://plato.stanford.edu/archives/spr2007/entries/goedel/.

Klein, J. (2004, September). Francis Bacon, In E. N. Zalta (Ed.), The Stanford Encyclopaedia of Philosophy, Fall 2004 Edition. Retrieved on January 10, 2006 from the website of The Stanford Encyclopaedia of Philosophy, http://plato.stanford.edu/archives/fall2004/entries/francis-bacon/

Kornblith, H. (1999), In defense of a naturalised epistemology, in John Greco and Ernest Sosa, The Blackwell Guide to Epistemology (pp. 158-169). Massachusetts: Blackwell.

Kornblith, Hilary (1986). Naturalising epistemology. In Naturalism and Rationality (pp. 115-134). Buffalo, New York: Prometheus Books.

Kripke, S. A. (1972). Naming and necessity. In G. Harman and D. Davidson (eds.), The Semantics of Natural Language. Dordrecht: Reidel [Reprinted as Kripke, 1980]. 
Kripke, S. A. (1980). Naming and Necessity. Cambridge, Massachusetts: Harvard University Press.

Kuhn, T. S. (1970). The Structure of Scientific Revolutions. Chicago: University of Chicago Press.

Kuhn, T. S. (1976), Scientific revolutions as changes of world view, in Sandra G. Harding, Can Theories be Refuted? (pp. 133-154). Boston: Reidal.

Kuo, Chia-Chen (2004). Research on impacts of team leadership on team effectiveness. In Journal of American Academy of Business, Vol. 5, No. 1-2; pp. 266-277.

Lahr, M.M. and Foley, R.A. (2004) Palaeoanthropology: Human evolution writ small. In Nature, Vol. 431, No. 7012, pp. 1043-1044.

Lakomski, G. (1995). Leadership and learning: From transformational leadership to organisational learning. In Leading and Managing. Vol. 1, No. 3, pp. 211-225.

Lakomski, G. (1999). Leadership, can we manage without it? Inaugural Professorial Lecture. Melbourne: Faculty of Education, University of Melbourne.

Lakomski, G. (2002, September), Distributed leadership: An idea whose time has come? Invited address, British Educational Leadership, Management and Administration Society, Annual Conference.

Lakomski, G. (2005). Managing Without Leadership: Towards a Theory of Organisational Functioning. Oxford: Elsevier.

Laudisa, F. and Rovelli, C. (2005). Relational Quantum Mechanics. In E. N. Zalta (ed.), The Stanford Encyclopedia of Philosophy (Fall 2005 Edition). Retrieved on 26 January 2007 from the website of the Stanford Encyclopedia of Philosophy at http://plato.stanford.edu/archives/fall2005/entries/qmrelational

Lave, J. and Wenger, E. (1991). Situated Learning: Legitimate Peripheral Participation. Cambridge: Cambridge University Press.

Lawson-Tancred, H. (1986). Introduction and notes. In Aristotle, De Anima (pp. 11116). Translated by H. Lawson-Tancred. London: Penguin.

Leadership: An overview (1997). Journal of Managerial Psychology, Vol. 12, No. 7/8, p. 435. 
Lehrer, K. (1996). Skepticism, lucid content and the metamental loop. In A. Clark, J. Ezquerro and J. M. Larrazabal, Philosophy and Cognitive Science: Categories, Consciousness, and Reasoning: Proceedings From the Second International Colloquium on Cognitive Science (pp. 73-93). Dordrecht, Netherlands: Kluwer Academic Publishers.

Leithwood, K., Day, C., Sammons, P., Harris, A., and Hopkins, D. (2006). Successful School Leadership: What It Is and How It Influences Pupil Learning: Nottingham: University of Nottingham.

Leithwood, K., Mascall, B., Strauss, T., Sacks, R., Memon, N. and Yashinka, A. (2007). Distributing leadership to make schools smarter: taking the ego out of the system. In Leadership and Policy in Schools, No. 6, pp. 37-67.

Levin, S. J. (1994). Semiotics, meaning, and discursive neural networks. In D. S. Levine and M. Aparicio IV, Neural Networks for Knowledge Representation and Inference (pp. 65-82). Hillsdale, New Jersey: Lawrence Erlbaum Associates.

Levinas, E. (1973). The Theory of Intuition in Husserl's Phenomenology. Translated by A. Orianne. Evanston: Northwestern University Press.

Lewin, K., Lippitt, R. and White, R. K. (1939). Patterns of aggressive behaviour in experimentally created social climates. In Journal of Social Psychology, Vol. 10, pp. 271-99.

Lewis, C. S. (1946), Miracles. Glasgow: Collins.

Lewis, R. (Ed.) (1992). Changing Curriculum. Geelong: Deakin University.

Lindsay, R. B. (1954). Operationalism in Physics Reassessed. In The Scientific Monthly, Vol. 79, No. 4, pp. 221-223.

Link, K. E. and Kreuz, R. J. (2005). Do men and women differ in their use of nonliteral language when they talk about emotions?. In H. L. Colston and A. N. Katz, Figurative Language Comprehension: Social and Cultural Influences (pp. 153-182). Mahwah, New Jersey: Lawrence Erlbaum Associates.

List, C. (2005). Distributed cognition: A perspective from social choice theory. In A. M. D. Schmidtchen and S. Voigt (eds.) Scientific Competition: Theory and Policy. Conferences on New Political Economy, 24, Tuebingen: Mohr Siebeck. Retrieved on 18 December 2006 from the website of London School of Economics at http://personal.lse.ac.uk/list/PDF-files/DistributedCognition.pdf. 
List, C. and Pettit, P. (2006). Group agency and Supervenience (Revised version, 11 January) Retrieved on 17 December 2006 from the website of the London School of Economics at http://personal.lse.ac.uk/list/PDFfiles/GroupAgencySupervenience.pdf.

Longford, E. (1969). Wellington: The Years of the Sword. Wiltshire: Redwood.

Lord, R. G. and Emrich, C. G. (2000). Thinking Outside the Box by Looking Inside the Box: Extending the Cognitive Revolution in Leadership Research. In Leadership Quarterly, Vol. 11,No. 4, pp. 551-579.

Lucas, J. R. (1996). Minds, machines, and Gödel: A retrospect. In P. J. R. Millican and A. Clark (eds.), Machines and Thought: The Legacy of Alan Turing, Vol. 1 (pp. 103-124). Oxford: Clarendon Press.

Luria, A. R. (1979). The Making of Mind. Cambridge, Massachusetts: Harvard University Press.

Lycan, W. G. (2005). A particularly compelling refutation of eliminative materialism. In C. E. Erneling and D. M. Johnson, The Mind as a Scientific Object: Between Brain and Culture. Oxford: Oxford University Press.

Lyngzeidetson, A. E. (1990). Massively Parallel Distributed Processing and a Computationalist Foundation for Cognitive Science. In The British Journal for the Philosophy of Science, Vol. 41, No. 1, pp. 121-127.

MacBeath, J. (2005). Leadership as distributed: A matter of practice. In School Leadership and Management, Vol. 25, No. 4, pp. 349-366

Mackellar, D. (2003). My Country. Retrieved on 15 February 2007 from the website of the estate of Dorothea Mackellar at http://www.dorotheamackellar.com.au/archive/mycountry.htm.

Maia, T. V. and McClelland, J. L. (2004). A reexamination of the evidence for the somatic marker hypothesis: What participants really know in the Iowa gambling task. In Proceedings of the National Academy of Sciences of the United States of America (PNAS), Vol.101, No.5. (pp. 16075-16080). Retrieved on 25 January 2007 from the website of the PNAS at http://www.pnas.org/cgi/reprint/101/45/16075.pdf.

Malcolm, C. (2003), 'Thoughts from South Africa', Lab Talk, Vol. 47, No. 5, pp. 3638.

Marchitello, H. (1997). Narrative and Meaning in Early Modern England: Browne's Skull and Other Mysteries. Cambridge: Cambridge University Press. 
Mark, D. M. (1993), Human spatial cognition, in D. Medyckyj-Scott and H. M. Hearnshaw (eds.), Human Factors in Geographical Information Systems, Belhaven Press, pp. 51-60.

Massachusetts Department of Education. (2004). Strategies for Distributed Leadership. Retrieved on January 3, 2006 from the website of the Massachusetts Department of Education: http://www.doe.mass.edu/eq/cslp/leadership/development.html

Masters, G. N. (2004). What makes a good leader? In Education Review, Vol. 7, No. 2, p. 12.

Matthews, G., Ziedner, M., and Roberts, R. D. (2002). Emotional Intelligence: Science and Myth. Cambridge, Massachusetts: MIT Press.

Matthews, R. (2002). A Longer View to Appease the Loonies. In The Age, 25 November, World, p. 11.

Mayrowetz, D., Murphy, J., Louis, K. S., Smylie, M. A. (2007). Distributed leadership as work redesign: Retrofitting the job characteristics model. In Leadership and Policy in Schools, Vol. 6, No. 1, pp. 69-101.

McGregor, C. (1950?), The Human Side of Enterprise, cited in Van Maurik (2001, pp. 14-17)

McKay, T. and Nelson, M., (2006). Propositional Attitude Reports, In E.N. Zalta (ed.), The Stanford Encyclopedia of Philosophy (Winter 2006 Edition). Retrieved on 8 January 2007 from the website of the Stanford Encyclopedia of Philosophy at http://plato.stanford.edu/archives/win2006/entries/prop-attitudereports/.

McLaughlin, B. and Bennett, K. (2005). Supervenience. In E. N. Zalta (Ed.). The Stanford Encyclopaedia of Philosophy, Fall 2005 Edition. Retrieved on January 3, 2006 from the website of the Stanford Encyclopaedia of Philosophy: http://plato.stanford.edu/archives/fall2005/entries/supervenience/

McMahon, J. T. (1972). The Contingency Theory: Logic and method revisited. In Personnel Psychology, Vol. 25, No. 4, pp. 697-710.

Mehra, A., Smith, B. R., Dixon, A. L. and Robertson, B. (2006). Distributed leadership in teams: The network of leadership perceptions and team performance. In The Leadership Quarterly, Vol. 17, No. 3, pp.232-245. 
Meilich, O. (2006). Bivariate Models of Fit in Contingency Theory: Critique and a Polynomial Regression Alternative. In Organizational Research Methods, Vol. 9, No. 2, pp. 161-193.

Merriam, S. B. (1988). Case Study Research in Education: A Qualitative Approach. San Francisco: Jossey-Bass.

Merton, R. K. (1957). Social Theory and Social Structure. New York: Free Press.

Middenhorp, C. (2004), Views from the top. In The Age, 15 May, Review, p. 8.

Miller, E. J. (1998). The leader with the vision: is time running out? In E. B. Klein, F. Gabelnick, \& P. Herr (Eds.), The Psychodynamics of Leadership. Madison, Connecticut: Psychosocial Press, pp. 3-25.

Milne, A. A. (1940). Winnie-the-Pooh. London: Methuene \& Co.

Minsky, M. (1983). Jokes and the logic of the cognitive unconscious. In R. Groner, M. Groner and W. F. Bischof, Methods of Heuristics (pp. 171-194). Hillsdale, New Jersey: Lawrence Erlbaum Associates.

Minsky, M. L. and Papert, S. A. (1988). Perceptrons: An Introduction to Computational Geometry, expanded edition. Cambridge, Massachusetts: MIT Press.

MIT Sloan. (2005). In Depth: Natural Disasters. Leadership: It Takes a Team. Retrieved on January 3, 2006 from the website of The Massachusetts Institute of Technology Sloan School of Management: http://mitsloan.mit.edu/newsroom/2005-indepth-disasters-leadership.php

Moberg, D. J. and Koch, J. L. (1975). A Critical Appraisal of Integrated Treatments of Contingency Findings. In The Academy of Management Journal, Vol. 18, No. 1, pp. 109-124.

Morris, W. E. (2001). David Hume. In E. N. Zalta (ed.), The Stanford Encyclopedia of Philosophy (Spring 2001 Edition). Retrieved on 2 August 2007 from the website of The Stanford Encyclopedia of Philosophy at http://plato.stanford.edu/archives/spr2001/entries/hume/.

Mosser, K. (1999). Bonjour, Kant and the a priori. In Disputatio, Vol. 7, pp. 40-53.

Moszkowski, A. (1993). Cited in White, M. and Gribben, J., Einstein: A Life in Science (p. 145). London: Simon and Schuster. 
Moustakas, C. (1990). Heuristic Research: Design, Methodology and Applications. Newbury Park, California: Sage.

Mulhauser, G. (2005). Supervenience. Retrieved on 3 January 2006 from the Mulhauser Consulting website at http://www.mulhauser.net/research/tutorials/supervenience/

Munson, C. E. (1981). Style and structure in supervision. In Journal of Education Social Work, Vol. 17, pp. 65-72.

Murphy, N. (2002). The problem of mental causation: How does reason get its grip on the brain? Science and Christian Belief, Vol. 14, No. 2, pp. 143-158.

Nadel, A. (1996). The Holocaust and Rodney King, memory and silence: Cliffs notes in the age of historical reproduction. In MFS Modern Fiction Studies, Vol. 42, No. 2, pp. 445-468.

Neserius, P. G. (1933). Isocrates' political and social Ideas. In International Journal of Ethics, Vol. 43, No. 3, pp. 307-328.

Newell, A. (1983). The heuristic of George Polya and its relation to artificial intelligence. In R. Groner, M. Groner and W. F. Bischof, Methods of Heuristics (pp. 195-244). Hillsdale, New Jersey: Lawrence Erlbaum Associates.

Nichols, S. (2004). Folk Psychology of Free Will. In Mind \& Language, Vol. 19, pp. 473-502. Retrieved on 4 July 2007 from the website of the University of Arizona at http://dingo.sbs.arizona.edu/ snichols/Papers/folkpsychologyoffreewill.pdf

Nichols, S. (2006). Folk intuitions on free will. In Journal of Cognition and Culture, Vol. 6, No. 1-2, pp. 57-86

Nida-Rümelin, M. (2006). Grasping phenomenal properties. In T. Alter and S. Walter, Phenomenal Concepts and Phenomenal Knowledge: New Essays on Consciousness and Physicalism (pp. 307-350). Oxford: Oxford University Press. Retrieved on 7 July 2007 from the website of Université de Fribourg at http://fns.unifr.ch/philocolor/Doc/MNR.Grasping\%20Phenomenal\%20Properti es, \%20neu\%209\%2005.pdf

Nisbet, J. (1999). Policy-oriented research. In J. P. Keeves and G. Lakomski, Issues in Educational Research (pp. 64-75). Oxford: Pergamon.

Nisbet, J. D. and Entwistle, N. J. (1970). Educational Research Methods. London: University of London Press. 
O'Connor, T. (2000). Causality, Mind and Free Will. In Philosophical Perspectives, Vol. 14, pp. 105-117.

Ödman, P-J. (1988). Hermeneutics. In J.P. Keeves (ed.), Educational Research Methodology and Research: An International Handbook (pp. 63-70). Oxford: Pergamon.

OECD (2001). Preliminary Synthesis, Second High Level Forum, Learning Sciences and Brain Research: Potential Implications For Education Policies And Practices, Brain Mechanisms And Youth Learning, Granada, Spain, 1-2-3 February 2001, Organisation for Economic Co-operation and Development, Center for Educational Research and Innovation.

Oppenheimer, J. R. (1966). Science and the Common Understanding. New York: Simon and Schuster.

Ormiston, G. L. and Sassower, R. (1989). Narrative Experiments: The Discursive Authority of Science and Technology. Minneapolis: University of Minnesota Press.

Packer, M. J. and Addison, R. B. (eds.) Entering the circle: Hermeneutic investigation in psychology. New York: State University of New York Press.

Parkdale Secondary College Gets Green Light for New Buildings (2006). Media Release from the Office of the Premier, State of Victoria, 29 August. Retrieved from the Victorian Government website of Victorian Legislation and Parliamentary Documents at http://www.legislation.vic.gov.au/domino/Web_Notes/newmedia.nsf/798c8b07 2d117a01ca256c8c0019bb01/5f5a5365e35f9cccca2571da0005b145!OpenDocu $\underline{\text { ment }}$

Parliament of Australia: Hansard (13 February 2008). Apology to Australia's Indigenous Peoples. Text of Prime Minister Rudd's speech to the House of Representatives. Retrieved on 15 March 2008 at the website of the Australian Parliament House at http://www.aph.gov.au/house/rudd_speech.pdf

Parsons, T. (1970). Social systems. In Grusky, O. and Miller, G. A. (eds.), The Sociology of Organisations (pp. 75-82). New York: The Free Press.

Paulos, J. A. (2000). I Think, Therefore I Laugh: The Flip Side of Philosophy. New York: Columbia University Press.

Pearl, J. (1996). Causation, action and counterfactuals. In A. Gammerman (ed.), Computational Learning and Probabilistic Reasoning (pp. 103-123).

Chichester, West Sussex: Wiley. 
Peifer, D. (2001). Commemoration of Mutiny, Rebellion, and Resistance in Postwar Germany: Public Memory, History, and the Formation of "Memory Beacons". In Journal of Military History, Vol. 65, No. 4, pp. 1013-1052.

Penrose, R. (1989). The Emperor's New Clothes. Oxford: Oxford University Press.

Penrose, R. (1994). Shadows of the Mind. Oxford: Oxford University Press.

Peters, L. H., Hartke, D. D. and Pohlmann, J. T. (1985). Fiedler's Contingency Theory of Leadership: An Application of the Meta-Analysis Procedures of Schmidt and Hunter. In Psychological Bulletin, Vol. 97, No. 2, pp. 274-285.

Pettit, P. (2004). Groups with minds of their own. In F. Schmitt, (ed.), Socializing Metaphysics (pp. 167-93). New York: Rowman and Littlefield. Retrieved on 17 December 2006 from the website of Princeton University at http://www.princeton.edu/ ppettit/papers/GroupMinds.pdf.

Pfeffer, J. (1977). The ambiguity of leadership. In Academy of Management Review, Vol. 2, No. 1, pp. 104-112.

Pitcher, G. (1965), Wittgenstein, Nonsense and Lewis Carroll, in Massachusetts Review, Vol. 6, No.3, pp. 591-611.

Pliska, P. (1997). Nonlocality and the principle of free experimentation. In A. Driessen and A. Suarez (eds.), Mathematical Undecideability, Quantum Nonlocality and the Question of the Existence of God (pp. 101-119). Dordrecht, The Netherlands: Kluwer Academic Publishers.

Pollock, J. L. (2006). Irrationality and Cognition. Paper presented at the Inland Northwest Philosophy Conference on Knowledge and Skepticism, held April 30-May 2, 2004, in Moscow, ID and Pullman, WA. Retrieved on 14 December 2006 from the website of John L. Pollock at http://oscarhome.socsci.arizona.edu/ftp/publications.html

Popper, K. (1972). Conjectures and Refutations: The Growth of Scientific Knowledge ( $4^{\text {th }}$ ed.) New London: Routledge and Kegan Paul.

Popper, K. R. (1968). The Logic of Scientific Discovery. London: Hutchinson.

Popper, K. R. (1982). Quantum Theory and the Schism in Physics (From the Postscript to the Logic of Scientific Discovery, Volume III). London: Hutchinson. 
Popper, K. R. (1998). The World of Parmenides: Essays on the Pre-Socratic Enlightenment. Edited by Arne F. Petersen with the assistance of Jørgen Meyer. London: Routledge.

Popper, K. R. and Eccles, J. C. (1983). The Self and It's Brain. London: Routledge and Kegan Paul.

Popper, M. (2004). Leadership as Relationship. In Journal for the Theory of Social Behaviour, Vol. 34, No. 2, pp. 107-125.

Porter, L. W. and McLaughlin, G. B. (2006). Leadership and the organisational context: Like the weather? In The Leadership Quarterly, Vol. 17, pp. 559-576.

Pountney, M. (2004). Mini-human Find: 'Hobbit' Skeletons in Lost World Cave. In The Herald Sun, 28 October, p. 3.

Pribham, K. H. (1994), Brain and the structure of narrative. In D.S. Levine and M. Aparicio IV (eds.), Neural Networks for Knowledge Representation and Inference (pp. 375-418). Hillsdale, New-Jersey: Lawrence Erlbaum Associates.

Putnam, H. (1975/1985) The meaning of 'meaning'. In Philosophical Papers, Vol. 2: Mind, Language and Reality. Cambridge University Press.

Quine W.V.O and Ullian, J.S. (1978), The Web of Belief, $2^{\text {nd }}$ edition. New York: Random House.

Quine, W.V.O (1986). Reply to Morton White. In L. E. Hahn and P. A. Schilpp, The Philosophy of W. V. Quine (pp. 663-665). La Salle Illinois: Open Court.

Quine, W.V.O (1995). From Stimulus to Science. Cambridge Massachusetts: Harvard University Press.

Quine, W.V.O. (1951). Two Dogmas of Empiricism. In Philosophical Review, Vol. 60, pp. 20-43.

Radick, G. (2005). What if...? Exploring Alternative Scientific Pasts. In New Scientist, No. 2513, p. 34. Retrieved on January 10, 2006 from the website of New Scientist: http://www.newscientist.com/channel/opinion/mg18725131.500

Ramberg, B. and Gjesdal, K. (2005). Hermeneutics. In, E. N. Zalta (ed.), The Stanford Encyclopedia of Philosophy (Winter 2005 Edition). Retrieved on 3 December 2006 from the website of the Stanford Encyclopedia of Philosophy at http://plato.stanford.edu/archives/win2005/entries/hermeneutics/. 
Ramsey, W. (2003), Eliminative Materialism. In E. N. Zalta (ed.), The Stanford Encyclopedia of Philosophy (Fall 2003 Edition). Retrieved on 13 January 2007 from the website of the Stanford Encyclopedia of Philosophy at http://plato.stanford.edu/archives/fall2003/entries/materialism-eliminative/.

Randerson, J. (2004, 29 July). Brain not body makes athletes feel tired. In New Scientist. Retrieved on 12 January 2007 from the website of New Scientist at

Reger, J. (2007). Where Are the Leaders? Music, Culture, and Contemporary Feminism. In American Behavioral Scientist, Vol. 50. No. 10, pp. 1350-1369.

Reuters (2006). Probe proves stem cell research faked. In ABC News Online, 10 January. Retrieved on January 10, 2006 from the website of the Australian Broadcasting Commission: http://www.abc.net.au/news/newsitems/200601/s1544890.htm

Ribeiro, A. C. (2003). Do Mirror Neurons Support a Simulation Theory of MindReading? Paper presented at the U.C. San Diego Graduate Philosophy Conference (April 12). Retrieved on January 6, 2006 from the website of the University of California, San Diego: http://aardvark.ucsd.edu/grad_conference/ribeiro.doc

Ricciulli, L., Lincoln, , P. and Meseguer, J. (1995). Execution-Driven Distributed Simulation of Parallel Architectures. Menlo Park, California: Computer Science Laboratory, SRI International. Retrieved on 10 March 2007 from the website of SRI International at http://www.csl.sri.com/papers/sri-csl-95-05/sricsl-95-05.pdf.

Ricouer, P. (1976). Interpretation Theory: Discourse and the Surplus of Meaning. Fort Worth, Texas: Texas Christian University Press.

Ricouer, P. (1981). Hermeneutics and the Human Sciences. Cambridge: Cambridge University Press.

Ritchhart, R. (2002). Intellectual Character: What It Is, Why It Matters, and How to Get It. San Francisco: Jossey-Bass.

Rizvi, F. (1986). Administrative Leadership and the Democratic Community as a Social Ideal. Geelong, Victoria: Deakin University.

Rizvi, F. (1991). Approaches to the Study of Educational Administration. Geelong: Deakin University. 
Roach, C. F. and Behling, O. (1984). Functionalism: Basis for an alternate approach to the study of leadership. In J. G. Hunt, D. M. Hosking, C. A. Schrisheim, and R. Stewart (eds.), Leaders and Managers: International Perspectives on Managerial Behaviour and Leadership. Elmsford, New York: Pergamon.

Rorty, A. (1980). Explaining emotions. In A. Rorty (ed.), Explaining Emotions (pp. 103-126). Berkeley: University of California Press.

Ross, D. (2005). Chalmer's Naturalistic Dualism: The irrelevance of the Mind-Body Problem to the Scientific Study of Consciousness. In C. E. Erneling and D. M. Johnson, The Mind as a Scientific Object: Between Brain and Culture (pp. 160175). New York: Oxford University Press.

Ross, D. (2006). Game Theory. In E. N. Zalta (ed.), The Stanford Encyclopedia of Philosophy (Spring 2006 Edition). Retrieved from the website of the Stanford Encylopedia of Philosophy at http://plato.stanford.edu/archives/spr2006/entries/game-theory/.

Ross, L., Rix, M. and Gold, J. (2005). Learning distributed leadership: Part 1. In Industrial and Commercial Training, Vol. 37, No. 2, pp. 130-137.

Rossi, P. (2005). Kant's Philosophy of Religion. In Edward N. Zalta (ed.), The Stanford Encyclopedia of Philosophy (Fall 2005 Edition), http://plato.stanford.edu/archives/fall2005/entries/kant-religion

Rost, J. C. (1995). Leaders and followers are the people in this relationship. In Wren, J.T. (Ed.). The Leader's Companion: Insight on Leadership Through the Ages (pp. 189-192). New York: Free Press.

Rumelhart, D. E., McClelland, J. L., and the PDP Research Group. (1986) Parallel Distributed Processing: Explorations in the Microstructure of Cognition, Vol. 1. Cambridge, Massachusetts: MIT Press.

Russell, H. (undated document). Chamberlain Case 1984. Retrieved on January 10, 2006 from the website of the Australian National University Faculty of Law at law.anu.edu.au/highcourt_project/Chamberlain\%20Case\%20rtf.rtf

Rustow, D. A. (1970). Philosophers and Kings: Studies in Leadership. New York: George Braziller.

Saab, D.J. (2003). Conceptualising space: Mapping schemas as meaningful representations. Masters Thesis. Cambridge, Massachusetts: Lesley University. Retrieved on 4 March 2004 from the website of D. J. Saab at http://www.djsaab.info/thesis/djsaab_thesis.pdf 
Sajer, G. (1999). The Forgotten Soldier: War on the Russian Front - A True Story. London: Cassell.

Salovey, P., and J.S. Mayer (1990). Emotional Intelligence. In Imagination, Cognition, and Personality, Vol. 9, No.3, pp. 185-211.

Sarros, J. C., Gray, J. H. and Densten, I. (2002). Australian Business Management Survey. Management Research Series, No. 1/2002. St. Kilda, Victoria: Australian Institute of Management.

Sashkin, M., and Garland, H. (1979). Laboratory and field research on leadership: integrating divergent streams. In J. G. Hunt, and L. L. Larson (Eds.), Crosscurrents in leadership (pp. 64-87). Carbondale, IL: Southern Illinois University Press.

Schins, J. M (1997). Mathematics: a pointer to an independent reality (Penrose's interpretation of the Gödel and Turing theorems). In A. Driessen and A. Suarez (eds.), Mathematical Undecideability, Quantum Nonlocality and the Question of the Existence of God (pp. 49-56). Dordrecht, The Netherlands: Kluwer Academic Publishers.

Schleifer, R., Davis, R. C. and Mergler, N. (1992). Culture and Cognition: The Boundaries of Literary and Scientific Inquiry. Ithaca, New York: Cornell University Press.

Schön, D.A. (1983). The Reflective Practitioner: How Professionals Think in Action. New York: Basic Books.

Seeger, R. J. (1954). Beyond Operationalism. In The Scientific Monthly, Vol. 79, No. 4, pp. 226-227.

Selleck, R. J. W. (1990). Tate, Frank (1864 - 1939). In Australian Dictionary of Biography, Vol. 12 (pp 169-172). Melbourne: .Melbourne University Press. Retrieved on 13 December 2006 from the online website of the Australian Dictionary of Biography (ADB), Research School of Social Sciences, Australian National University at http://www.adb.online.anu.edu.au/biogs/A120189b.htm

Semler, R. (1994). Maverick! The Success Story Behind the World's Most Unusual Workplace. London: Arrow.

Seneca (2004). Letters From a Stoic: Epistula Morales ad Lucilium. Selected and Translated with an Introduction from R. Campbell. London: Penguin. 
Senge, P. M. (1990). The Fifth Discipline: The Art and Practice of the Learning Organization. New York: Doubleday.

Sergiovanni, T. J. (1992). Moral leadership: Getting to the Heart of School Improvement. San Francisco: Jossey-Bass.

Sergiovanni, T. J. (2001). Leadership: What's in It for Schools?. London: Routledge.

Sergiovanni, T. J. (2004). Building Community in Schools. San Francisco, California: Jossey-Bass.

Shakespeare, W. (1984). Othello. Cambridge: Cambridge University Press.

Shakespeare, W. (1997). Macbeth. Melbourne, Victoria: Nelson International Thomson Publishing.

Shakespeare, W. (2000). Julius Caesar. Retrieved on 9 December 2006 from the website of The Literature Network at http://www.online-

literature.com/shakespeare/julius_caesar/5/.

Shamier, B. (1999). Leadership in boundary-less organizations: disposable or indispensable? European Journal of Work and Organisational Psychology, Vol. 8, No. 1, pp. 49-71.

Shannon, C. (1948). A Mathematical Theory of Communication. In The Bell System Technical Journal. Vol. 27, pp. 379-423, pp. 623-656.

Shirer, W. L. (1960). Rise and Fall of the Third Reich. Place of publication not given: Book Club Associates.

Simon, H. A. (1996). Machine as mind. In P. J. R. Millican and A. Clark (eds.), Machines and Thought: The Legacy of Alan Turing, Vol. 1 (pp. 81-102). Oxford: Clarendon Press.

Smith, D. W. (2005). Phenomenology. In E. N. Zalta (ed.), The Stanford Encyclopedia of Philosophy (Winter 2005 Edition). Retrieved on 11 July 2007 from the website of the Stanford Encyclopedia of Philosophy at http://plato.stanford.edu/archives/win2005/entries/phenomenology/

Snowden, D. J. (2007). A leader's framework for decision-making. In Harvard Business Review, Vol. 85, No. 11, pp. 68-76. 
Sosa, E. (1999) Scepticism and the internal/external divide, in John Greco and Ernest Sosa, The Blackwell Guide to Epistemology (pp. 145-157). Massachusetts: Blackwell.

Southworth, G. and Doughty, J. (2006). A fine British blend: effectively combining research and practice. England's National College for School Leadership offers an exemplary model of leadership training. In Educational Leadership, May 2006, pp. 51-54.

Spillane, J. P. (2006). Distributed Leadership. San Francisco: Jossey-Bass.

Spillane, J. P., Camburn, E. M., and Parega, A. S. (2007). Leadership and policy in schools. In Leadership and Policy in Schools, Vol. 6, No. 1, pp. 103-125.

Spillane, J. P., Halverson, R. and Diamond, J. B. (2001). Investigating School Leadership Practice: A Distributed Perspective (pp. 23-28). Educational Researcher, Vol. 30, No.3.

Starkey, D. (2000). Elizabeth. London: Chatto and Windus.

Starratt, R. J. (1990). The Drama of Schooling: The Schooling of Drama. London: Falmer Press,

Starratt, R. J. (1993). The Drama of Leadership. London: Falmer Press.

Starratt, R. J. (1994). Building an Ethical School: A Practical Response to the Moral Crisis in Schools. Washington: Falmer Press.

Stent, G. S. (2005). Epistemic dualism. In C. E. Erneling and D. M. Johnson, The Mind as a Scientific Object: Between Brain and Culture (pp. 144-159). New York: Oxford University Press.

Steup, M. (2005). Internalism and externalism. In The Analysis of Knowledge. In E.

N. Zalta (Ed.), The Stanford Encyclopaedia of Philosophy (Winter 2005

Edition). Retrieved on January 5, 2006 from the website of the Stanford Encyclopaedia of Philosophy: http://plato.stanford.edu/archives/win2005/entries/knowledge-analysis/

Stich, S. and Nichols, S. (1992). Folk psychology: simulation or tacit theory? In Mind \& Language, Vol. 7, No.1, pp. 35-71. Retrieved on January 2, 2006 from the website for the Rutgers University Research Group on Evolution and Higher Cognition: http://ruccs.rutgers.edu/ArchiveFolder/Research\%20Group/Publications/Sim1/ $\underline{\text { sim1.html. }}$ 
Stich, S. P. (1996). Deconstructing the Mind. Oxford: Oxford University Press.

Stogdill, R. M. (1948). Personal factors associated with leadership: A survey of the literature. In Journal of Psychology. Vol. 25, pp. 35-71.

Stogdill, R.M. (1974). Handbook of leadership: A Survey of Theory and Research. New York: Free Press.

Stoljar, D., (1997). Deflationary Theory of Truth. In E. N. Zalta (ed.). The Stanford Encyclopedia of Philosophy (Fall 1997 Edition). Retrieved on 1 October 2006 from the website of The Stanford Encyclopedia of Philosophy at http://plato.stanford.edu/archives/fall1997/entries/truth-deflationary/.

Strauss, C. (1997). Research on cultural discontinuities. In C. Strauss and N. Quinn, A Cognitive Theory of Cultural Meaning (pp. 210-251). Cambridge: Cambridge University Press.

Strauss, C. and Quinn, N. (1997). A Cognitive Theory of Cultural Meaning. Cambridge: Cambridge University Press.

Strube, M. J. and Garcia, J. E. (1981). A Meta-Analytic Investigation of Fiedler's Contingency Model of Leadership Effectiveness. In Psychological Bulletin, Vol. 90, No. 2, pp. 307-321.

Suarez, A. (1997). Nonlocal phenomena: physical explanation and philosophical implications. In A. Driessen and A. Suarez (eds.). Mathematical Undecideability, Quantum Nonlocality and the Question of the Existence of God (pp. 143-172). Dordrecht, The Netherlands: Kluwer Academic Publishers.

Sweller, J. (2006). Natural information processing systems. In Evolutionary Psychology, Vol. 4, pp. 434-458. Retrieved on 31 January 2007 from the website of Evolutionary Psychology at http://www.epjournal.net/filestore/ep04434458.pdf.

Tannenbaum, R. \& Schmidt (1973). How to choose a leadership pattern. Harvard Business Review, May-June.

Tarski, A. (1983). Logic, Semantics, Metamathematics: Papers from 1923 to 1938. Translated by J. H. Woodger. Indianapolis: Hackett.

Tarski, A. (1994). The semantic conception of truth. Originally published in Philosophy and Phenomenological Research, 1943, Vol. 4, pp. 341-375. Reprinted in P. Horwich (ed.), Theories of Truth (pp. 285-314). Aldershot, Hants: Dartmouth. 
Tate, F. (1895). The training of teachers. Australian Schoolmaster and Literary Review (June, pp. 220-1). Reprinted in A.G. Austin and R.J.W. Selleck (1975). The Australian Government School 1830-1914 (pp. 71-2, 139-144). Carlton: Pitman.

Taylor, M. (1982). Community, Anarchy and Liberty. Cambridge: Cambridge University Press.

Tettamanti, M., Buccino, G., Saccuman, M., Gallese, V., Dana, M., Scifo, P., Fazio, F., Rizzolatti, G., Cappa, S., and Perani, D. (2005). Listening to action-related sentences activates fronto-parietal motor circuits. In Journal of Cognitive Neuroscience, Vol. 17, pp. 73-281. Retrieved on 31 January 2007 from the website of Universita Degli Studi Di Parma at http://www.unipr.it/arpa/mirror/pubs/pdffiles/Buccino\%20et\%20al\%202005.pd f.

Tichy, N. M. and Devanna, M. A. (1986). The Transformational Leader. New York: Wiley.

Tieszen, R. (1998). Gödel's path from the incompleteness theorems (1931) to phenomenology (1961). In The Bulletin of Symbolic Logic, Vol. 4, No. 2, pp. 181-203.

Tikhomirov, O. K. (1983). Informal heuristic principles of motivation and emotion in human problem solving. In R. Groner, M. Groner and W. F. Bischof, Methods of Heuristics (pp. 153-170). Hillsdale, New Jersey: Lawrence Erlbaum Associates.

Timperley, H. S. (2005). Distributed leadership: Developing theory from practice. In Journal of Curriculum Studies, Vol. 37, No. 4, pp. 395-420.

Todd, R. B. (1976). The four causes: Aristotle's exposition and the ancients. In Journal of the History of Ideas, Vol. 37, No. 2, pp. 319-322.

Tolstoy, L. (1991). War and Peace. Translation by Louise and Aylmer Maude. New York: Oxford University Press.

University of Florida Scientist: 'Brain' in a Dish Acts as Autopilot, Living

Computer. (Oct 21, 2004). Retrieved January 11, 2007, from the website of the University of Florida:

http://www.napa.ufl.edu/2004news/braindish.htm

Utecht, R. E. and Heier, W. D. (1976). The Contingency Model and Successful Military Leadership. In The Academy of Management Journal, Vol. 19, No. 4, pp. 606-618. 
Valanides, N. and Angeli, C. (2008). Distributed Cognition in a Sixth-Grade Classroom: An Attempt to Overcome Alternative Conceptions about Light and Color. In Journal of Research on Technology in Education, Vol. 40, No. 3, pp. 309-336.

Van der Waerden, B. L. (1983). Inspiration and thinking in mathematics. In R. Groner, M. Groner and W. F. Bischof, Methods of Heuristics (pp. 69-78). Hillsdale, New Jersey: Lawrence Erlbaum Associates.

Van Inwagen, P. (2000). The Eighth Philosophical Perspectives Lecture: Free Will Remains a Mystery. In Philosophical Perspectives, Vol. 14, pp. 1-19.

Van Maurik, J. (2001), Writers on Leadership. London: Penguin.

VBMTAB (Victorian Bushwalking and Mountaincraft Training Advisory Board) (1978). Bushwalking and Mountaincraft Leadership. Clayton, Victoria: Victorian Bushwalking and Mountaincraft Training Advisory Board.

Vecchio, R. P. (1979). A Dyadic Interpretation of the Contingency Model of Leadership Effectiveness. The Academy of Management Journal, Vol. 22, No. 3, pp. 590-600.

Vickers, J. (2006). The Problem of Induction. In E. N. Zalta (ed.), The Stanford Encyclopedia of Philosophy (Winter 2006 Edition). Retrieved on 13 January 2007 from the website of Stanford Encyclopedia of Philosophy at http://plato.stanford.edu/archives/win2006/entries/induction-problem/.

Vvidis, I. K. (2002). Distributed cognition and educational practice. In Journal of Interactive Learning Research. Spring, pp.11-19.

Vygotsky, L.S. (1962). Thought and Language. Cambridge, Massachusetts: The MIT Press.

Vygotsky, L.S. (1978), Mind in Society, Cambridge, Massachusetts: Harvard University Press.

Walton, D. (1996). Fallacies arising from ambiguity. Dordrecht, The Netherlands: Kluwer Academic Publishers

Wang, H. (1996). A Logical Journey: From Gödel to Philosophy. Cambridge, Massachusetts: MIT Press.

Waterhouse, L. (2006). Multiple Intelligences, the Mozart Effect, and Emotional Intelligence: A Critical Review. In Educational Psychologist, Vol. 41, No. 4, pp. 207-225. 
Watkins, P. (1986). A Critical Review of Leadership Concepts and Research: The Implications for Educational Administration. Geelong, Victoria: Deakin University.

Waugh, S. (2005). Out of My Comfort Zone: The Autobiography. Camberwell, Victoria: Viking.

Weber, R., Camerer, C., Rottenstreich and Y., Knez, M. (2001). The illusion of leadership: Misattribution of cause in coordination games. In Organization Science, Vol. 12, No. 5, pp. 582-598.

Wells, A. (1993). Parallel Architectures and Mental Computation. In The British Journal for the Philosophy of Science, Vol. 44, No. 3, pp. 531-542.

White, M. \& Gribben, J. (1993). Einstein: A Life in Science. London: Simon \& Schuster.

Whitney, W. D. (ed.) (1899). Century Dictionary: An Encyclopaedic Lexicon of the English Language. London: The Times.

Wiener, N. (1948). Cybernetics, New York: John Wiley and Sons.

Wight, S. (2003). Australian Schooling: A History of Social Control. In Otherways Magazine. 30 October. Retrieved on 7 August 2007 from the website of the Home Education Network at http://www.homeed.vic.edu.au/2003/10/30/australian-schooling-a-history-of-social-control/.

Wilkie, J. S. (1950). The problem of the temporal relation of cause and effect. In The British Journal for the Philosophy of Science, Vol. 1, No. 3, pp. 211-229.

Williams, M. (1986). Do we (epistemologists) need a theory of truth? (pp. 223-242). In Philosophical Topics, XIV, No. 1. Reprinted in P. Horwich (ed.), 1994, Theories of Truth. Aldershot, Hants, England: Dartmouth.

Williams, M. (1999). Groundless Belief, $2^{\text {nd }}$ Ed. Princeton: Princeton University Press.

Wilson, D. (1998). Accident Prevention, Vol. 44, No. 9. Retrieved on 15 July 2007 from the website of the Flight Safety Foundation at http://www.flightsafety.org/ap/ap_sept88.pdf

Wood, M. (2005). The fallacy of misplaced leadership. In Journal of Management Studies, Vol. 42, No. 6, pp.1101-1121. 
Woods, P. A., Bennett, N., Harvey, J. A. and Wise, C. (2004). Variabilities and dualities in distributed leadership. In Educational Management Administration and Leadership, Vol. 32, No. 4, pp. 439-457.

Wooton, L. M. (1977). The Mixed Blessings of Contingency Management. In The Academy of Management Review, Vol. 2, No. 3, pp. 431-441.

Wreden, N. (2005). Language: Churchill's key to leadership. In Becoming an Effective Leader (pp. 185-194). Boston, Massachusetts: Harvard Business School Press.

$\mathrm{Xu}$, L. H. (2006) The optimisation of learning in science classrooms from the perspective of distributed cognition. Thesis (M.Ed.). Melbourne: University of Melbourne, Dept. of Science and Mathematics Education.

Yin, R. K. (1981). The case study crisis: Some answers. In Administrative Science Quarterly, Vol. 26, No. 1, pp. 58-65.

Young, J. O. (2001), The Coherence Theory of Truth. In E. N. Zalta (ed.), The Stanford Encyclopedia of Philosophy (Summer 2001 Edition). Retrieved on 7 July 2007 from the website of the Stanford Encyclopedia of Philosophy at http://plato.stanford.edu/archives/sum2001/entries/truth-coherence/.

Young, P. T. (1943), Emotion in Man and Animal: Its relation to attitude and motive. New York: John Wiley and Sons.

Zagzebski, L.(1999). What is knowledge? In J. Greco and E. Sosa, The Blackwell Guide to Epistemology (pp. 92-116). Massachusetts: Blackwell.

Zhang, J., \& Norman, D. A. (1994). Representations in distributed cognitive tasks. Cognitive Science, Vol. 18, No. 1, pp. 87-122.

Zimmerman, D. (2001). Thinking with your hypothalamus: Reflections on a cognitive role for the reactive emotions. In Philosophy and Phenomenological Research, vol. 63, No. 3, pp. 521-541.

Zwartz, B. (2005). Let's have a proper scientific debate. In The Age, 18 August, p. 13. 


\section{Appendix 1: Interview B5 Transcript}

\section{Interviewer: Andrew Hill (author) \\ Interviewee: Sarah", Principal of Eucalypt ${ }^{\#}$ Primary School ("pseudonym)}

Andrew: How long have you been in the leadership position in this school?

Sarah: Three years.

Andrew: Please describe the scope of your current responsibilities in the school?

Sarah: I think the main purpose of my role is educational leadership in the school. Provision of educational opportunities for children would be number one. I'm Executive Officer of School Council obviously. There is obviously the management role of the financial aspects of the school and the organisational aspects of the school. I also see communication with the school community as a prime role that I have in the school, communication with the wider community and beyond (internationally as well). Student welfare is a major role; it's a specific responsibility of mine, but it is also a role that I see as a very important role for principals to have. So, student and staff welfare, personnel issues ... Educational leadership, I suppose, is the one we would like to be in full control of but we're being driven... I suppose the Government directed drive is towards more management (which is disturbing to say the least).

Andrew: What do you hope to achieve in your current position as a leader?

Sarah: I suppose a school with a shared vision, a school where children are happy to come to. My personal vision is for the school to grow, that the enrolments increase to enable us to be particularly viable. As an enrichment of the school (rather than our numbers going up); we're also looking at numbers going up because of the arts program that we have; people coming for specific reasons like the environmental 
aspect and the multicultural aspect. The other thing that I'm very much into, I suppose, is the staff; to have a happy staff, a contented staff. There are a lot of things I can't control but I can control the daily work conditions of the staff here. I feel that if we can work together to help them to build daily work conditions that are good, then I feel there is potential to achieve a lot. The staff are very important.

Andrew: With that in mind, what sorts of data or information do you use to assess your progress?

Sarah: It's still the very rigid stuff. In statistical terms, it's data really only related to that data which is appropriate for the school annual report. There's a lot of anecdotal data that I take in as leader of the school, and that is because I have a team approach to leadership and management. There are lots of forms of feedback that I give out and receive from staff that enable me to gauge the feelings, the concerns that the staff share. So, I have a leadership team that is not supposed to be top heavy in terms of the designated roles, it just happens to be that way this year. I have a leadership and management process where the teachers can come in if they have a particular concern. Staff meetings are very open processes; they're meant to be chaired by a variety of people, [with] the minutes taken by a variety of people. So, there is a lot of collaboration and consultation. There are staff meetings, area meetings and team meetings. So, there is a whole team approach around the school in terms of leadership and management. The minutes of meetings are kept for me, I've got them in a folder over there, so I get a lot of feedback. The non-teaching staff have their own fortnightly integration aide meeting plus non-teaching staff and they know that I'll read the minutes. If they have any concerns, then I'll get back to them.

Andrew: How does the information influence your personal achievements and the way that you operate?

Sarah: Hugely. I've tried to be a different type of leader to the one that I was when I first took over. I think I've had a dramatic change, I'm a very verbal person. I think that is why I became a leader. The things that caused me to have leadership qualities are the things that I have to, at times, hold back on as the leader of the school, such as, chairing meetings for example. I very rarely chair a meeting, I never chair a meeting in front of the staff (they think I'm appalling). I am a person that is 
all over the place, goes off track, so I've had to quieten down and listen, use a lot more of my ears than my mouth. I've changed, I've attempted to become far more aware and listen to people more than I was. I came from the classroom straight into a principal role. For six months I was as acting assistant principal.

Andrew: That was a big jump?

Sarah: Yes it was. So, I was really a teacher who had a lot to say. It's taken, probably, three years to cut down, to find the appropriate time to say things and listen to the staff more than perhaps talking to them. I think that has changed. I think I've got a very big personality, that can be used to the staff's detriment or ... I think I wear my emotions a lot on the outside, people know whether I'm in a good mood or a bad mood. Again, It's something I've tried to be very aware of - to not walk in with a bad look on my face; to make sure that the smile is there and to be open to a lot of changes in that area.

Andrew: Would you say the school community here shares common values and beliefs about education and schooling?

Sarah: It's been very hard to know what the school community here believes until probably this year, this is a very non-participatory school community, a very multicultural community, and a very diverse community, a diverse community where it was hard to draw people together. But, I have to say for this year, the parent participation has increased dramatically for a few reasons. I would say now we do have a shared vision, I think that people are coming to the school because they clearly understand what the issues are, what we are trying to achieve for the school. So, yes I think we do have [shared values and beliefs]. That's not to say that parents don't individually have different values and different beliefs about the way their children should learn. But, I can understand that more now and I think we take that into consideration, that people want to contribute and participate in school life in a different way. Whereas when I first took over I wanted them to look at things the same way and got no response at all. 
Andrew: With respect to your answer to the last question what would you say the shared values are and what areas of disagreement or non-agreement would there be?

Sarah: The shared values, I believe, are that parents and myself believe the children should have more educational opportunity. I don't try to sell the school as an arts school or a technology school. My insistence to parents is that we have a strong focus on literacy and numeracy early in the mornings, through the staff we have allocated, reading recovery teachers and early intervention programs. But, the eight key learning areas are seen as a total package for us and the children. The multicultural diversity of the school is something that I stress with interviews with parents: 'If you are not interested in your child being in the classroom with other children from other countries then we don't want you either!' It was to our detriment again three years ago. People would not come here because there were lots of Asian children in the school. Now, I have people coming from a number of nearby localities, it's a plus that we're multicultural. And there are middle class AngloSaxon Australian families who want their children to be part of the multicultural community (which is wonderful). So, the multicultural aspect is important. I think children wanting to learn, children feeling confident to learn, children being listened to, children having confidence to accept challenges and take risks is a very important aspect that I think parents appreciate as well. I think they want the children to do the very best they can. I think the parents share that belief about school and what school is for.

Andrew: So parents, when they send their children here, know that is the underlying value [operating] here?

Sarah: Yes. I think one of the other things is that the whole school approach to student welfare and discipline is highly focused as well, that we have a uniform approach to the way the welfare of the students is handled. Self-esteem is the high focus of that, but there is [also] a whole school approach to welfare and discipline in the school. 
Andrew: Would you say the school community here has a shared vision of what you hope to achieve together in the school, and if so, what would you say that shared vision is?

Sarah: No, I don't think they do have a shared vision at the moment. I think that as we work towards our next school charter, again, whilst I've had increased parent participation, It's because we have more articulate parents coming into the school. The school is not just culturally diverse, but it's socio-economically diverse as well. The new arts community that's come in is very focused in on the environment and environmental issues and arts focus. I heard a parent say last night, 'Last night was absolutely fantastic, when are they going to start doing some real work again?' So, that family has very high expectations of their child and 'this dilly-dallying with the arts is very nice but it's not really what we're here for!' All our Asian community were here very strongly last night, instrumental music and so on. But, I think there are those who see school as still back in the dim dark ages of reading, writing and arithmetic and the arts community are wanting almost the reverse to happen, the focus on these areas the children will learn naturally ...

Andrew: Those two groups stand out do they?

Sarah: I think yes. It is not to say that the multicultural community is not interested in the arts at all. I just think they have a stronger focus on traditional ways of learning. They don't see themselves as necessarily needing to participate in school life because they work. Whereas the arts community see themselves as very much in here; hands-on with children planting trees and involved in Earthcare day, in environment day. And there's the other group, a smaller group of parents who are in desperate, dire need. Just last week we had three or four families with no homes to go to. So, children with no lunch, no food, no rent, nothing. We're talking about absolutely rock bottom. So there is that sort of adversity in the school. And I think those parents really care. They're probably more concerned that their children are in a caring environment. They know they'll get support from me, they know I'll be able to help them to seek out agencies to assist them. So there's that whole welfare issue 
there. I believe they see the school as very supportive institution. We're a very unusual school in terms of the complexity of our population.

Andrew: This next question is designed to follow on the idea of there being a shared vision or some sort of idea of where the school is heading and the question is: 'What sorts of data or information does the school collect?' So, perhaps you can reword that to suit the context here.

Sarah: I think It's an interesting question because the arts community are involved in environmental issues and they have asked me how can they be involved, how can they influence the direction of the next school charter. Because, they have their agenda of instrumental music, choir, full performance, recycling, they want policies made about the whole gamut of environmental issues, they want to know how they can influence and be part of the school charter, working on what they want to do. They want to feed information in but don't really want to do the work, and I think that is probably going to be the direction of a lot of schools now. They need to get the information and outsource. They need to learn how to get the correct information from parents and that's one of the things that I said to them at the meeting the other night: 'Your opinions are valued but they may not necessarily reflect the opinions of parents in the wider school community'. We talked about the correct way of sampling. They will go and ask all of the environmental friends in school for their surveys and that would have to be what the school charter would be based on for next year. I explained that that would be a distorted sample, that we possibly would be looking at, say in the whole school community; that we would be making it available (which they weren't really happy about) because they certainly wanted to make their mark made. So, I'm still very much in ignorance, I suppose, of how to correctly question the community so that we do get the opinions of all, and how to get that back in a succinct way for us to put together; because I don't want to end up with a school charter document that only serves one-quarter of the school community. We've made that very clear: that the silent - that our non-English speaking background parents (they may not care, that's fine) - I need to know at least whether they're happy with the way things are going. So, I don't think that group realises there are other groups in the school that need to be taken into 
consideration. So, that was a long answer! The short answer to that question after a long statement there is [this]: It's still very anecdotal in terms of vision, so it will be individual surveys. The environmental group have been asked to come up with some questions that they would like to be asked. We'll look at them and if they are too slanted (we want open-ended questions not, 'Do you want $\$ 30,000$ spent on the junior school and the senior school on trees for next year?'). [It's], 'Get the issues to us and we'll try to devise the questions.'

Andrew: That's a difficult balance that you have to strike there.

Sarah: It is very much so, for these are the people who are increasing our enrolments. The arts community, the environmental community has moved in and to knock them back. One of the questions was, 'We've spent $\$ 50,000$ on the school playground this year, you're not going to not let us spend more money next year Sarah?' And that's what you'll get. They have come into the school council and been hysterical, very emotive, they're very passionate people about what they want.

Andrew: I suppose you're dealing with that with an underlying set of values that everybody's voice is important?

Sarah: Absolutely. The recycling ... they would like plastic removed, children not to be bringing any kind of extra paper. With a multicultural community, they need to be aware that we don't have extra paper around the school and their children bring rice in plastic containers. We've also got to be very aware of the food packaging, that children don't all bring the same kinds of food to school.

Andrew: You've touched on this next question, so if there is any more to add to that please do so. The question is, how do you analyse this data to monitor the achievements of the school's vision?

Sarah: The staff probably do $99.9 \%$ of the data analysis. I believe it really isn't their role and that in the future I will be outsourcing it as it is. The only reason being is we had a student teacher here last term; it was an older student who came into the 
school with a statistical background and he saw a completely different set of outcomes to the ones that we looked at. He was purely looking at it from the statisticians' point of view. He had the blend of both; I mean he opened our eyes up to a whole lot of information we had not even noticed. It's not good for us either; teachers are not trained to do that. So, as we require more information I think we will be outsourcing it.

Andrew: How has this (or any) information you have collected at this point (you might want to project yourself into the future too); how has this influenced (or how will it influence) the school's achievements and the way the school operates?

Sarah: The last two school annual reports have greatly influenced the direction of the school. I think the last one, the 1996 one, is definitely moving towards a greater focus on the arts, in fact I'm sure it's going to be one of our priorities for the next school charter, literacy is the other. We're becoming better at analysing student achievement in those areas because we've got that information up on the computer. But literacy is also another area that's coming through and it's coming through from parent surveys and teacher feedback. They are very keen and have found enthusiasm for their own professional development and to follow through with literacy. I think we will be seeing maths and English now as a rotational thing. We'll be .... maths, English, maths, English, with maybe the arts or science (or whatever, a [third] one) being attached almost on a two year basis.

Andrew: Could you please choose, in your mind, one key team you're an active member of in this school, and could you tell me the name of that team please?

Sarah: It's the Education Planning Committee [EPC].

Andrew: Could you please describe how that team operates?

Sarah: It's the leadership team of the school. It happens to be, this year, the assistant principal Carolyn, the leading teacher two Margaret, and the senior and junior coordinators. In the past it's just been a couple of teacher reps. But, these 
people actually wanted to be on it when I asked about (the previous year) what responsibilities the people would like the following year. Both of these people happened to put down the EPC so they're on it also because they personally chose to be on it. It's the leadership, the big vision stuff, it's the where-are-we-heading-tonow. We don't bog ourselves down so much with excursion and management issues and administrative issues, It's more the wider educational focus, why-are-we-doingthings. We reflect on what's happening around the school, which teams of teachers are working well, which teams aren't. It's looking at whole staffing issues, looking at the big curriculum issues. It's the only team that enables us to sit down and reflect and look at where we're heading. It's not a past [thinking], it's a forward thinking group.

Andrew: As a team, what sorts of data or information do you use to judge your progress in achieving the aims of the team?

Sarah: Because each person happens to be from either the senior or junior area of the school, we draw on junior and senior area issues. Because we're a small school, it seems that we do a lot of the things that we do together. So, it's feedback from teachers, really, that draws us together, I think, and helps us to make decisions about which way the school's going. Probably, not this committee but a past committee, where I had teachers come from a school elsewhere, we were looking more at research - we were actually looking at really big picture stuff. She was studying, I was studying, and the Education Planning Committee was really very forward thinking a lot of the time. But two staff members have since left and it's probably not as easy to get into really big philosophical discussions than we would have in the past.

Andrew: Can you give me an example?

Sarah: We were doing research into composite classrooms and there would be weeks of discussion about the pros and cons of multi-age classrooms. That's just off the top of my head. But really, it was the whole concept of the Schools of the Future: What was it trying to achieve? What is self-management? What were the pros and 
cons of it? What would the impact on staff be? Why are we doing this? Gail had been overseas, she was with Brian Caldwell doing overseas work, so she had been looking at the local government schools. So we would sit in on these meetings to discuss the schools in England and Canada and how it was different to schools here and were they better or weren't they?

Andrew: Thinking back to the achievements of the team and the data or information did you use to judge your progress, how has this information also influenced the teams work?

Sarah: What my aim is, is to continually improve the quality of teaching and learning in the school. I say that as a two-sided thing because unless teachers are involved in continual improvement through professional development, through selfreflection, through working with each other, then I don't believe that student improvement will happen. Again, I can say that student achievement has increased dramatically here in the last three years because I think teachers have picked up on computer education, that we've had a stronger focus on literacy, teachers have been looking at the way they teach it, how children learn. They see all of it as being responsible, as a total group, for the progress of the children at school. So, I think that is the focus of Education Planning Committee as well, that continual improvement of both teachers and children.

Andrew: Can you describe what this team will be hoping to achieve in the future?

Sarah: Again, the big picture stuff, looking forward. Where we're going, where are we now and where are we heading. I think one of the short term things that has happened is (three years ago the teachers didn't have annual plans, they really taught off the top of their heads) in the last three years we've now achieved the annual planner, the termly planner, the weekly planner. At the end of this year, in Term 4 we're now going to be looking at two-year scope and sequence charts. So, I think that the staff are saying, 'Yes, I've been planning for twelve months, I know where I'm heading with the kids. I know what needs to be done to get the children to where they need to get to and then I can plan back in the term and back to the week'. So in 
the future, I hope that our team helps the staff to look into [the] longer term, starting with a two year scope and sequence chart, certainly in the areas of SoSE [studies of society and environment] and technology and science and so on. So, if a teacher is new in the school, we can give them a longer term plan, 'This is our school, this is the plan for three years'. I think [that] making sense out of the data, we have to be very careful about this data collection: Who is it for, is it really going to make a difference to children? I think that the fact we have in place an assessment and reporting policy and program is excellent, and I believe it has contributed to improved student outcomes. We have to be very careful about spending teacher time sitting down with too much data. Who is the audience?

Andrew: With respect to the previous questions, how would you summarise your own ways of thinking and reasoning about your work?

Sarah: I'm an all over the place thinker. I'm a person, I suppose, who thinks in terms of potential, what could be. I like to see anything as a challenge. I'm a person who gets very bored with short term things, I know that something is going to be implemented ... I've moved way beyond ... that's one of the problems I have as a principal with the staff. It's lovely to have a blend of people in your staff and also in your leadership team who have different ways of viewing [things]. Carolyn's very strategic, very organised, I'm probably seen as disorganised and my ideas are offbeat. I completely lose track; I can be thinking of lots of different things at the same time and recall them, unfortunately for the staff, at any point in time in the conversation (when we can be talking about something completely different and something else will have popped into my head). That is one of the problems I have to learn to deal with. So, I think I am a person who looks for a challenge when someone says ... well three years ago someone said there is no way known we could get a Japanese LOTE [languages other than English] person into the school; we got one! Anything is possible, if it's worth trying, if it's worth going for, we can get it. I think that's how I think.

Andrew: Finally, in this school do you hold conversations with others in which you share views and develop knowledge about each other's assumptions? 
Sarah: All the time! I think, all the time. Not just through formal meetings but also through the communication with staff, the feedback that I get from them, and hopefully the feedback I give to them, through written notes to them. We didn't have a staff meeting on Monday night. We decided to go around the local park for a walk. That time I walked around with three teachers. In fact one of the teachers was supposed to be taking formal staff meeting minutes and he decided take the minutes of the meeting from the conversations of people going around the park, which he linked to the CSF [Curriculum and Standards Framework] outcome statements (it was just sensational). But, that was another way of communicating with staff in a completely different atmosphere; and yet they valued the fact there was no meeting because of the school concert. Those who had the time to walk around the park thoroughly enjoyed it and sat down and we had coffee and cappuccinos after it. I felt that the aim of the evening had far exceeded what I wanted to achieve, which was to get people to think this week was not going to be a very hard week for them; a meeting on Monday night would have been a total waste of time. I think we try to think creatively about how we keep morale up; we know that the workload isn't going to go, it's going to increase; change is here, so lets manage it. Carolyn works incredibly; her relationship with the staff is sensational; the changes that have occurred in the school couldn't have happened without her; the staff have enormous respect for her. 


\section{Appendix 2: Interview B6 Transcript}

\section{Interviewer: Andrew Hill (author) \\ Interviewee: Joan", School Council President of Eucalypt ${ }^{\#}$ Primary School (\#pseudonym)}

Andrew: How long have you been in the current leadership position at this school?

Joan: Since the beginning of the year.

Andrew: Can you describe please the scope of your current leadership position?

Joan: Well, basically I'm School Council President; my main practical function is to actually chair the school council meetings and make sure they run smoothly, to time and to cover all the agenda items. I liaise fairly strongly with the principal. We communicate quite closely about the issues within the school that she thinks I need to know about, and I talk to parents about issues that they may want to speak to me about. If there is a problem we try and get it resolved quickly to everybody's mutual agreement (or whatever). If I can't do that I would put it in Sarah's hands to, maybe, do something which is meant to happen formally through the Department of Education (or whatever), or through welfare within the school. And, of course, talking to other school council members about issues they may have, and just as a parent of the school.

Andrew: That's a fair range of issues you have to undertake.

Joan: It's a fairly responsible position, and it can be quite a powerful position. I don't actually see it like that myself, because I think that if you looked at it like that you could actually, if you wanted to, exert that power. It's hard to describe how I see it, but I think that you could possibly, if you really wanted to, exert that power to 
push the school in directions that you wanted (which may not be in the best interest [of] everybody in the school). So I try to keep away from that kind of perception.

Andrew: What do you hope to achieve in your current position as leader?

Joan: I don't think I really have any strong perceptions of what I hope to achieve, apart from to do the job well, and to the best of my ability, and to keep most of the people happy most of the time (I think).

Andrew: What sorts of data or information do you use to assess your progress as a leader?

Joan: Data ... nothing concrete, nothing on a piece of paper. It would one hundred percent verbal feedback from Sarah, other school council members, people talking to me in the schoolyard - anecdotal stuff, definitely.

Andrew: How has this information influenced your personal achievements and the way you operate?

Joan: It probably hasn't influenced me personally but it does influence what I see as day to day priorities or issues to be addressed.

Andrew: Would you say the school community here shares common values or beliefs about education and schooling?

Joan: Quite strongly, I think. What we're trying to do here is give the school community opportunities to voice those directions and ideas. From the feedback we get, from meetings and talking to parents, this has been [the case]. Also, I think it's a lot easier for parents to choose a school now because parents do shop around - and the fact that schools now have a school charter which you can go away and read. It gives you a clear understanding of the directions the school's heading in; what it hopes to achieve and what the priorities are... How well the school achieves that? - 
[well] I think you have to look around the school and talk to other parents. I think our school community is moving fairly well in the same direction.

Andrew: You've hinted that the direction is a commonly held one, or commonly understood one. What would you say the shared values are - if you were asked to identify them? And, would you say there are any areas of disagreement or nonagreement?

Joan: To actually label the values is difficult. A lot of them are difficult issues like our multicultural things; like sharing and caring. There's a very strong emphasis on the environment in the school. We do a lot of work to help with that goal. We (the parents) have done a lot of native planting on the oval, which will grow and provide shade. There is a lot of emphasis on shade. I think also that the school population is changing and we're getting a lot more enrolments from people who are very strong in the arts. There is a very strong push for the school community to move into the arts. A lot more drama and that sort of teaching in the school. That is coming up very strongly. We're now deciding what our school charter priorities will be for the next three years.

[Regarding areas of agreement or disagreement], in my mind, I don't really see anything that would really stick out, I think, because of the whole school approach. As an example, the same rules are carried right through each grade. Each child knows the consequences of certain behaviour and that they're always going to be the same no matter what teacher you've got, what grade you've got. So there's a consistency; not where on one day you're being told something and on another day you're being told something else.

Andrew: Would you say the school community here has a shared vision of what you hope to achieve together for the school. If so, what would you say that shared vision is?

Joan: That's quite a hard question. A large part of the school community would not have a vision. They would be happy to go along with the decisions made for 
them by the school [council]. But on the other hand, there is a group of parents who have a strong vision, it's an environmental vision. They would like to have hundreds of thousands of dollars spent on the actual outside school environment. But, at the same time they are very driven, passionate, articulate parents who need to get part of their vision because that motivates them and keeps them passionate. So within the bounds of the money that we do have - that we can free up - we've given that group of parents quite a substantial amount of money linked to the school environment; and we'll be giving a bucket of money to them at the end of next year too; to do some more things. So, in terms of a common vision, I think it's a very much a 'go with the flow' thing; and that side of it is strongly guided by the school charter. I think, in terms of the way schools go now, it is quite structured for the school community in terms of [the] emphasis on what the school charter says (even though you can change it). Unless something goes drastically wrong, I don't see us changing the school charter because it's what a group decided on at that particular time. It has to last for three years.

Andrew: What sorts of data or information does the school collect?

Joan: Well obviously there's [the] LAP [Learning Assessment Program] and, I think, it's called the Standards Framework

Andrew: Curriculum and Standards Framework?

Joan: Yes, the tests that they do on that in the grades. The parent surveys, the school annual report is quite important in terms of the data that's collected and what's analysed in terms of what we have to do formally through the school. Something we did start to do last year in our school report (in the annual report) was, in terms of the parent and teacher survey (there is a teacher survey as well). We not only included the physical data but we included anecdotal comments as well, so that you could get an idea of what exactly parents were thinking, because you can't tell that from a graph. Off the top of my head, there's other sorts of data that we do collect. Absences, injuries, suspensions, all that sort of thing, legal requirements, on 
the computer. But that's off the top of my head because I don't have a lot to do with actual data.

Andrew: How do you analyse this data to monitor the achievement of the school's vision?

Joan: I haven't a clue [laughter]! Carolyn and Sarah handle a lot of that and I've heard them say on several occasions that the systems for gathering data have changed so much even from our '95 report to our '96 report. It's like comparing apples with oranges and it's virtually impossible to do it. But it was easier this time to compare '96 results with '97 results when we did our Triennial Review, because the data was collected using the same systems. It didn't really mean a lot to me until we actually sat down with the reviewer and looked at the data to see whether our kids were performing at, above or below the State norms. It was more interesting for me looking at it as a parent than as School Council President. I mean it was great for me as the President to see that the school was achieving well, but I looked at the data more emotionally as a parent.

Andrew: How has this information influenced the school's achievements and the way the school operates (from your understanding)?

Joan: I don't quite understand that question, so I'll just interpret it the way I think. We have an emphasis on English in the school. From our results, like the LAP [Learning Assessment Program] against the Curriculum and Standards Framework and so on, showing that, generally, over the school we are performing at or above the State average. As one of our priorities in the school charter, we make English one of those priorities so that we do what the staff have set up, put more money into the English program. We have reading recovery, which my daughter does. But, once reading recovery is finished, there's nothing else. They really do fall in a hole those children that need help above and beyond reading recovery. By making English a school charter priority, it means that we can free up money so that we can - I think it's called guided reading or something - to get that program up and running. It definitely needs a lot of money to get it going because of the resources and the 
materials. Lots of PD [Professional Development] is needed for the staff to get it up and running, but it would be fantastic. We do need specialist help above and beyond reading recovery. We're talking about results aren't we and how it guides the school? We could have looked at making maths a priority but maths was also achieving at or above the State norms pretty well across the board. So we decided that it would be OK to let the maths program continue as it is and make the English a priority. I think that was based on the results.

Andrew: Could you please choose one key team that you're an active member of in the school. What is the name of that team?

Joan: I'm on the finance sub-committee of the School Council with several others.

Andrew: Could you please describe how this team operates and what you've achieved together recently?

Joan: We meet once a month, the week before School Council. We keep a record of our minutes and any recommendations. What we've achieved recently is we have set the curriculum budgets for next year, and had them ratified by School Council. That's probably our biggest achievement.

Andrew: As a team, what sorts of data or information do you collect to assess you progress in achieving what you've achieved recently?

Joan: The bottom line would be our bank balance, that it's a credit balance; that there's enough money to go around. That would be what judges how well the finance sub-committee performs.

Andrew: How has this information influenced the team's work and what do you hope to achieve in the future? 
Joan: A bottom line thing right now, is that we've actually had to cut money from our curriculum budget because we've not got as much money as we used to have.

Andrew: So, that's influenced the way you allocated money to curriculum issues, for example?

Joan: Yes. Another thing that it's done is it's made the sub-committee look long and hard at the number of families who pay their (I hate this phrase) 'voluntary levy' (but really, that is what it's called). We have a very poor level of contribution and next year ... we have made a decision through School Council to take a much harder line approach to families to make that contribution.

Andrew: With respect to the previous questions, how would you summarise your own ways of thinking and reasoning about your work or role as school council president?

Joan: Well I try to listen to what everybody has to say, and try to let as many people as possible have a say about issues as I can, within the time constraints of the School Council meetings. I try not to be too emotional about the position and to take the practical, reasonable approach. Sometimes it's hard to do, particularly when you're a parent.

Andrew: In this school, do you hold conversations with others in which you openly share views and knowledge about each other's assumptions?

Joan: Yes, absolutely. There's one issue at the moment for which a lot of parents have approached me, and we've had quite open discussions about this issue. The issue has not been resolved yet, but I'm working towards it. I hope that parents know that they can talk to me at any time, because I'm quite approachable. I'm not in the school grounds a lot because I work but I try to be here as often as I can. 


\section{Appendix 3: Interview B7 Transcript}

Interviewer: Andrew Hill (author)

Interviewee: Carolyn ${ }^{\#}$, Assistant Principal of Eucalypt ${ }^{\#}$ Primary School ("pseudonym)

Andrew: How long have you been in your current leadership position in the school?

Carolyn: It would be fifteen months and it's an acting position, it hasn't actually been advertised.

Andrew: Could you please describe the scope of your current leadership responsibilities in the school?

Carolyn: Yes, I photocopied this [displaying a document]. These are the role statements for assistant principal and I was going through this (I think this was an old one; 1995). I am involved in the allocation of budgets, positions of responsibility and other resources in the school - I don't have a final say obviously; Sarah, as principal, would have the final say, but in my role as AP [Assistant Principal], I have a role in that) - the delivery of curriculum in the school to some extent; discipline matters beyond the classroom in the area come to the AP and then on to the principal, so I'm involved in that; the overall management of the school, and obviously, I take over when Sarah is absent. [Also], allocation of allowances (I'm involved in discussions about that too); selection of staff; issues of responsibility; grades for next year; leave and all that sort of thing; some curriculum proposals that go to School Council (if they go through the Curriculum Sub-committee because that's the one I'm on) but not all of them. I'm involved in decisions for short-term replacement teachers (and usually interviews for those teachers); the School Code of Conduct (I was involved in that process, but so are a lot of other staff too, so it crosses over); area coordinators (selection of those); specifically, my role in professional development, the budget, Personal Professional Development Appraisal Process all teaching and non-teaching 
staff; I'm responsible for assessment and reporting, 'P to 6' in all eight KLAs [Key Learning Areas]; and I have a full teaching load in Prep.

Andrew: That's a fair swag of responsibilities! What do you hope to achieve in your current position here Carolyn?

Carolyn: It is an acting position and my real love is curriculum and being in the classroom. So, I enjoy the acting position of Assistant Principal and I took it on as a challenge, but I'm not sure that it is something that I would apply for in another school. It's more, for me, a learning curve. So, when I took on the acting position, what I wanted to achieve were the tasks I was given, I'm very much a person who wants to start and finish a task. If I'm responsible for PD [Professional Development], I want to make sure that it's done properly. Assessment and reporting: If I have to devise a new report format then I do that and then implement it. So, I want to make sure that I do the job that I'm given by the principal and those sorts of tasks. I guess the other thing was to be seen as a leader in the school. When I first took over the position, it was a jump from a level one teacher (a level one teacher to an AP is a huge jump!). One of the things I wanted to achieve was to be seen as a leader not just a person acting in that position. But a lot of people feel that they could come to me with problems or advice and the parents could do as well. That seems to have happened. Also, to improve curriculum delivery in classrooms through the PD process and handle conflict better (in other words, to be more assertive).

Andrew: What sorts of data or information do you use to assess your progress in achieving these things?

Carolyn: I'm a person who documents things thoroughly, so I've got the documentation that shows that I've completed those tasks that I was talking about and that comes through the PRP [Performance Recognition Program] process. Although, in the leadership role, in the PRP process, I'm meant to be assessed on my substantive position, which means it wouldn't involve any of the AP stuff. We have slotted it in because I need to be accountable for it, so I have documentation for 
things like the PD and reports. The other thing I've done, from a suggestion from Sarah, is this idea of feeling like a leader, just in a journal form, to jot down when there has been, perhaps a conflict resolution or something like that; then I just write it in, just like a diary form. That's basically the 'data' as such. The data on student achievement and responses from the Parent Opinion Survey specific to 'Student Reporting,' which form part of the Annual Report and Triennial Review, also help me to reflect on my progress.

Andrew: How has this information influenced your personal achievements?

Carolyn: I've always been a very organised person, so I guess it's the importance of organising and timetables and things and to start sticking to those things. How does this information influence my personal achievements... What do you mean?

Andrew: Well that relates back to the [earlier] question in terms of what you hope to achieve.

Carolyn: Because my achievement's changed or ...

Andrew: Yes; or has it contributed towards your achievements in any way at all?

Carolyn: Which information?

Andrew: The sorts of information you collect in your journal entries, the things you mentioned in your answers to question two.

Carolyn: I think it just proves that I've achieved what I've expected to do! So, it's accountability. It just proves that I've done it and, if I haven't, I just sit back and reflect and make sure I start to achieve what I've set out to do. The data from the Triennial Review shows improvement in student achievement so curriculum delivery is improving. I have been more assertive (mainly with difficult staff members). A supportive colleague confirmed my more assertive behaviour after observing me over a period of time. 
Andrew: Thanks, that's fine. Would you say the school community here shares common values and beliefs about education and schooling?

[There was a short break in the interview here whilst a staff member made a brief inquiry with Carolyn]

Carolyn: I think we do have it, as an expectation of the community, that we're able to teach reading and writing, especially the literacy and numeracy side of it; but we have a very diverse community and we have very different ideas coming in. We have the Chinese community who believe in sitting down and doing work, getting lots of homework. Then we have another group, I guess they think that the reading and writing should happen because that's our job, but want to talk about the environment and all that sort of thing. And then there is another section of the community that is very much into the arts. We as teachers have the eight KLAs to cover and we believe we have to cater in all those areas, although literacy and numeracy, probably, we give a priority. The parents have come and looked at it from different angles, so it's very hard to say that we share the same common values or beliefs. I think it varies, depending upon where they're coming from.

Andrew: With that in mind, what would you say the shared values are? You have hinted at this already (the literacy and numeracy), but are there others you might like to add in terms of what the shared values are? You have mentioned the areas of disagreement or non-agreement, but you might like to add to that.

Carolyn: I think, definitely, the literacy and numeracy; and I think also tolerance, because it's a very multicultural school. I think there is an expectation that we do things at school to make children more tolerant of each other. So, I think that's another value that is expected of a school like us. I don't know of any major areas of disagreement, that I can think of; I might think of some later when I've had a think about it more. 
Andrew: Would you say the school community here has a shared vision of what you hope to achieve together for this school? If so, what would you say that shared vision is?

Carolyn: At the moment with the community that we've got now, the vision is the literacy and numeracy thing will happen, but the vision is we will become a real environmental, arts, multicultural community school, almost (that's the impression I get. I don't see as many parents as Sarah, but she's probably hearing different things); the environment, especially in the junior school (from the parents), it's that sort of thing. They're keen about the literacy and numeracy and they think that's going to happen (that's a given anyway) but they can see the kids going out into the community and performing and getting into worms and the environment and all that sort of thing. I think that's the position; we've become a very green, tolerant school and all that other learning happens anyway.

Andrew: What sorts of data or information does this school collect?

Carolyn: From a classroom teacher's point of view or a parent's? The annual report, we collect the same data as [every other school]: The parent survey; there's the teacher survey; there's the student achievement in maths and English; there's the student accidents, staff absences, student absences, all those things which Sarah would collect. There's a lot more, but we collect all that sort of data.

Andrew: Is there other data, or information (it doesn't have to be what you might strictly see as data)?

Carolyn: Classroom teachers collect information all the time just by talking to parents about what might have happened on the weekend with a child that might explain why they're grumpy today. There is a lot of that incidental, anecdotal [information] that classroom teachers (and specialists probably) would get; and Sarah on the welfare side, there is a lot of data for welfare children (through formal weekly meetings) that is filed; specific data for the integration children, gifted children, 
student learning improvement plan. So, there's specific data for those children, there's a lot of that anecdotal [information], especially in the junior area.

Andrew: How do you analyse this data to monitor the achievement of the school's vision?

Carolyn: Well, all the formal analysing is done for the annual report with special graphs (whatever they are).

Andrew: Bar and whisker graphs?

Carolyn: Yes, bar and whisker graphs! So, all that data helps us to monitor the achievement of the curriculum, the student learning outcomes and all of that sort of thing. To be perfectly honest, the parent opinion survey is [for] analysing so that we provide a brief comment about what the figures are telling us; but I don't know that the figures have actually changed ... well it has changed the way we've talked, we've got policies on homework as a result of the data from the parents and their opinion about certain things. So, we've introduced homework. I guess that if the parent opinion survey showed the parents are interested in the environment and all that sort of thing, then our vision might change through the analysis of that data. That's how that data will be reflected in the school charter, I guess, the school vision.

Andrew: Do you have any more specific analysis of that data that come to mind?

Carolyn: No, we've had a lot of trouble analysing the data correctly. No, that's something quite new to us, I think.

Andrew: You have mentioned this already, but you may wish to expand on it. How has this information affected the school's achievements and the way the school operates?

Carolyn: The information from the data? 
Andrew: No, not necessarily from data, it could also be anecdotal information.

Carolyn: Since we've been doing the annual report and the parent surveys, and things like that we have introduced policies, we've got the homework policy, we've got - [which has] just gone through School Council - an elimination of harassment policy for students and we're going to write one for adults to cover teachers. So, that's changing the way in which the school operates. What we've introduced is a two-hour literacy and numeracy block, the first two hours of the day. I was looking at the data, kids results from reading and it was pretty ordinary; so timetabling has changed, we try not to have specialists in the morning; so that's changing the way the school operates. Probably [because of] the feedback from the teachers, we've stopped timetabling meetings before school and things like that.

Andrew: Could you please choose in your mind one key team you're an active member of in this school... and could you tell me what the name of that team is please?

Carolyn: Do you want me to choose a different one to Sarah?

Andrew: No, just one that you feel is [important] to you, from your perspective.

Carolyn: The Education Planning Committee.

Andrew: Could you describe how this team operates and what you've achieved together recently?

Carolyn: That team is made up of the principal, assistant principal, leading teacher two, and the two area leaders, and it meets fortnightly. Initially when it was thought about, it was when the management committee was non-existent but we thought it was important to have some sort of leadership team, a formal meeting happening, so we called it the Education Planning Committee [EPC]. It's meant to be a forum for discussion about educational issues as well as specific staffing things. So, 
yes, meeting fortnightly it has achieved an enormous amount. You mean specifically this year or just over the last couple of years?

Andrew: I'll leave it up to you to decide.

Carolyn: Through the Education Planning Committee a lot of the decisions are made for staffing. We introduced a staff conference that we ran two years in a row. We contributed to the agenda and organised all the activities that were going to happen. A couple of specific matters where the EPC is responsible, is for our regular staff meetings; one is usually admin[istration] and at the next one, it will be curriculum. The focus for the staff meeting is discussed at Education Planning what we think the needs of the school are at the time. We talk about the PRP process, talk through various issues - issues that are bothering the staff; they can ask a member of the Education Planning Committee to bring it up. If they're not confident in doing it on their own, they can go through an EPC member and we can discuss it.

Andrew: As a team, what sorts of data or information do you use to judge your progress in achieving your aims?

Carolyn: I don't think we've ever sat down and analysed it in the Education Planning Committee.

Andrew: You may not have necessarily gone through a formal process of analysis, but you may have used that information to guide your thoughts. Did that influence the way you went about addressing certain issues?

Carolyn: I honestly don't know. I'm really not sure. Is that about the leadership team that makes the decisions?

Andrew: In terms of ... the team may have set out to achieve a certain thing.

Carolyn: Like a staff conference? 
Andrew: Yes; how do you judge your progress in doing that with the information that came back?

Carolyn: Yes; for a lot of the things we do, like the staff conference and curriculum days, we usually have an evaluation that we come back and discuss. We look at them and take from that ideas for the next meeting. That's very formal and specific to the conference. Curriculum days, we might sit around and discuss who is going to be in what grade next year, tell the staff of our decision and then ask for feedback, just verbally, and then come back and re-look at things The only real formal feedback is a formal evaluation after ... It will be interesting to work out a way to see how effective we are. That's something to think about!

Andrew: With respect to the previous questions, how would you summarise your own ways of thinking and reasoning about your work?

Carolyn: How do I think and reason about my work? Just, how I'm able to reflect on my ...

Andrew: Yes, you personally. What is it that makes you tick in terms of your professional role as assistant principal and teacher?

Carolyn: Often, when I'm confronted with issues and think, 'How am I going to react or deal with this situation?' I think of my mentor, she was a leading teacher two here, and is now principal at another school, and who is just fantastic. So, I guess I do often think of her: How would she react? How would she handle this situation? And I sometimes ring her. I think, because I am only in an acting position (this is all very new to me) that certainly when she was here, I relied on her heavily. Now that she's gone and I've jumped up, I still ring her; so I guess I'm still relying on her a bit. I'm a very calm person, in some ways I'm almost the opposite to Sarah, which makes for a better combination, I think. I'm always a very calm sort of person, because Sarah is more a reactionary, which is a 'plant' if you've ever done that, is it Belbin? One of those. 
Andrew: A Belbin Inventory?

Carolyn: I don't know if it was a Belbin, did they have plants?

Andrew: I can't remember! Yes, I think it is Belbin.

Carolyn: She's a plant. A very small percentage of people are plants. That's why Sarah never chairs a meeting, she goes all over the place. Whereas, I like to be structured and organised and take things [one] at a time. That's how I go about my work, too. So, that's we how we work, that's the way I am. Does that answer that?

Andrew: It does answer that.

\section{Carolyn: Good!}

Andrew: The last question, do you hold conversations with others in which you share views and develop knowledge about each other's assumptions?

Carolyn: Yes. Lots of things that happen are confidential, so that obviously makes it difficult; but I have to have someone that I can talk about my frustrations and share opinions with, and that depends on what it's about; a team member, a person I teach with, who I'm with all the time, and can talk openly to; and it depends on what the issue is; who I think, in the school, is going to tell me what I want to hear! Again, my mentor when there are things happening. 


\section{Appendix 4: Interview B8 Transcript}

\section{Interviewer: Andrew Hill (author) \\ Interviewee: Margaret ${ }^{\#}$, Leading Teacher, Eucalypt ${ }^{\#}$ Primary School ("pseudonym)}

Andrew: How long have you been in your current leadership position in that school?

Margaret: This is my second year.

Andrew: Could you describe, please, the scope of your current leadership responsibilities in the school?

Margaret: I lead the charter in the school and I'm part of the planning team, the EPC [Education Planning Committee] (so I do leadership work with Sarah and Carolyn in that way). With the computers, now, I assist staff throughout the school, if they have trouble with their computers. I write about charter priorities for computer and time allocations. I take the curriculum staff meetings (any curriculum based staff meetings). I'm in charge of curriculum for the whole school (curriculum coordinator for the school). I'm also the leader of the curriculum coordinators' network of the area. [I'm] in charge of the English KLA [Key Learning Area] in the school as well.

Andrew: What do you hope to achieve in your current position as a leader?

Margaret: I hope that I give other people confidence to come to me, that I can help them with whatever... I know, so far anyway, the difference between last year and this year is huge with the confidence I have this year - I didn't think that I would have had [it] last year. I find that all the different challenges that I have been faced with, I've been able to take up and go on with (which I've been happy with). I know that the staff, now, can come and speak to me, (which they do, which is good) about whatever. Whether it would be hardware with their computer, or whether it would be 
about something else, or issues. Because I also go around and look at everybody's work programs and their planning and the trouble they have, I help them out with that as well.

Andrew: What sorts of data of information do you use to assess your progress [towards] your achievements?

Margaret: Do you mean check lists that I keep for kids?

Andrew: In terms of what you are hoping to achieve generally.

Margaret: Probably through my PRP [Performance Recognition Program] process where I have to have things documented. I have kept records of meeting with people. I've kept records in the form of checklists when I've gone through and checked people's work to make sure that things have been covered. I keep records through minutes from meetings, agendas from meetings, things like that. I write down notes if I feel things needed to be noted. So, I keep records of all [sorts of] different things.

Andrew: How has this information influenced your personal achievements and the way you operate?

Margaret: It's good in that I can look back on things and manage to reflect on things. I can also use it to build on; or introduce or work out new things to do, different ways of helping people.

Andrew: Would you say the school community here shares common values or beliefs about education and schooling?

Margaret: Yes, I think they do. Even though they might have differences, I think we are in a very - what you could term - 'arty', gifted community in that way. They are very, very supportive of the school and what the school is doing. I know last year, I was on School Council, and the School Council totally support the school and what 
the school is doing. I know the support I have in my classrooms from parents with what's going on just in the classroom. They're quite happy with that. I know, when we are having school functions now, the difference even within a year. At the start of last year (or the end of the year before I visited the school) they had a school picnic at the end of the first year I came (which was when I first came to visit) and had about six people here, whereas now we (the school) is having anything with the community (the community is right behind it) and we would have a couple of hundred people. So, it's a big turn around I've noticed. I've been lucky enough, to see it from the start of last year with the new parents (mainly through the Prep) that came in last year, that have brought their friends, they've got other people through the school. There is a very nice community (I think) that is operating around in this school. The green rangers (with what they're doing with the environment of the school grounds) have done a lot to up lift the image, I suppose, of the school. I think the school [community] as a whole (before I got here) were working very hard to increase their profile in the [local] community, and I think that's all paying off now. I've been able to see the changes, the groundwork that has already been put in.

Andrew: If there are any areas where there are not shared values or beliefs what would you say the areas of disagreement or non-agreement are (that's only if there are any)?

Margaret: I can't think of any. I think where the community wants something the school has gone out of its way to try and accommodate that. I know, especially at the start of the year, the parents wanted a music program. But it was impossible, because we didn't have the staffing available. Through different things that have happened through the year, we've managed to offer and start up musical things within the school and I think that's been seen as important to them. Even if you go back to the Green Rangers Project, backing that with the school and the school funds, I think that goes to help show them we're doing things.

Andrew: Would you say that school community here has a shared vision (I suppose today, a shared vision is what you hope to achieve together for the school) and if so what would you say that shared vision is? 
Margaret: I'd say the shared vision is for (probably) a whole education (a rounded education). Not just necessarily an academic education but all the different areas. And opening up as many different areas for their children to be exposed to as possible.

Andrew: What sorts of data or information does this school collect?

Margaret: All sorts of data! You name it; we have to minute it, we write it down. I've never seen data collected like it before. You name it, you will find something written on it. For any meeting there are minutes; everywhere!

Andrew: So that would include assessment data and policies?

Margaret: You name you'll find it, nothing is left out.

Andrew: How do you analyse this data to monitor the achievement of the schools vision?

Margaret: Usually it can be monitored in teams, it can monitored as EPC [Education Planning Committee] - through the planning committee.

\section{Andrew: EPC being?}

Margaret: Being Sarah, Carolyn, myself and the two junior and senior school coordinators. I'd say most data would probably go through them and then they're just sent out into areas (depending on what the data is); that's what would happen, I'd say, to the data.

Andrew: How does this information influence the school's achievements and the way the school operates?

Margaret: It makes people look at it, go through it, check what needs to be done, with the data and then act on it from there. So, action research. 
Andrew: Could you please choose one key team you are an active member of in the school and could you tell me what the name of that team is please?

Margaret: The key team would be my [Grade] Five-Six Area Team. It's the senior area team and that's probably where we do the most work. That's the person who I work with the most, because we are doing the planning for the children and working out everything for the two grades.

Andrew: So, is it just the two teachers, you and the other Five-Six teacher?

Margaret: There is a senior area, which is grade three to six and this is just taking it into the five and six area.

Margaret: Breaking down from the top ones, because I mean you've got the EPC, you've got your staff working as a staff (which we do). Then we work as a senior area, then the little team, which is the one that I have the most contact with that would be the [key team].

Andrew: Now can you please describe how this team operates and what you've achieved together recently?

Margaret: This year has been turmoil plus! Only because the man I was working with, Brian, went on sick leave partly through last term (right at the beginning of the last term). Consequently, I had to work with a number of different people in the room, trying to keep the grade settled, so it was very important to work with them. Now that there is another person in the room, Eric, I have to work closely with Eric to make sure he can see what's going on and letting him know what has happened, where we are going from here. [There is] lots of planning that we have to do, from excursions and getting ready for our camp next year down to what's been taught each week in the room. Working through that and how to assess it and all the assessment that is coming up. Making sure that he is aware of what school policies are on all the different things and working through that. 
Andrew: It must be pretty difficult getting together to talk when you've got such a busy classroom schedule?

Margaret: We'll plan times for meetings (which are Wednesday night after school) but otherwise we would talk every day. I make sure (especially because he is new) I go and see him every day. After school [we] talk about things, whenever we can anyway, or during a recess or a lunch.

Andrew: I know how hard it is to come into a school for the first time; it's very difficult.

Margaret: We've done all our major planning for this term together which was good, so that he has a very good idea of what to do. It makes him feel a lot better. Also, I'm working with him on the computer literacy because he hasn't used computers before. We're trying to do a lot of our planning on the computer with him, so he can see [how the computer can be used]. I've got him now taking the laptop home and using the laptop, he learned how to use it last week; it was good.

Andrew: As a team, what sorts of data or information do you use to judge progress in achieving your aims?

Margaret: We could use the assessment data from the grade, if you like. Going through, first of all, from our whole school policy on assessment, to what we should be collecting, to all the actual things that you just need to do in your classroom anyway. Just making sure that we both have all those things done and up to date. It's good to have somebody to bounce off how you interpret things, and I think because we both working on the same CSF level (we both have children within the level) it's very good to be able to discuss that level together.

Andrew: What things? Your assessment strategies or ... 
Margaret: Apart from the strategies, it's more [like] going back to evaluate where the students are, where you think they are on the CSF [Curriculum and Standards Framework] basically, or how you think they work within that area, I suppose.

Andrew: How has this information influenced your work as a team and what do you hope to achieve together in the future?

Margaret: Through the assessment, it would influence the planning; so that if we felt that areas weren't been covered - weren't being successfully achieved - we would do something about that, we would change how the program was been operated; we would implement something that was different (new) to try and help the children meet those aims that we were trying to achieve.

Andrew: Just think back to the previous questions because this relates to [the next two questions] in some way. With respect to those questions, how would you summarise your own ways of thinking and reasoning about your work?

Margaret: I suppose I think about what needs to be done, ways of achieving it, and go about using those ways. Then look back on what I have done to judge how successfully what I wanted to do is achieved, and then from that, plot to go on. Work to either improve or keep going with those things.

Andrew: In this school do you hold conversations with others in which openly share views and develop knowledge about each other's assumptions?

Margaret: Yes, the staff here are excellent, I think. I think everybody here is very similar in their thoughts, aims and how they're working. Even when everybody is at different levels you can do it, whether you wanted to do the structure or teaching different grade levels. Everybody can still come together. I think everybody here, basically, has this shared goal, this year, more so than last year. Last year it was all over the place, but this year everybody seems to be working together as a team and they see the school operating as a team. 
Andrew: Why do you think this happened?

Margaret: I think Sarah worked very hard last year to get people's confidence in herself and how she operates as a team. I think it all reflects back on Sarah because I think she is a lot more approachable to everybody this year and people feel that they can speak to her. I don't know, maybe it's combination of the people that we have in the school this year. Last year for a change ... normally I think what's been policy in this school is to up and move everybody (just to get you a variety of experiences in different grade levels) so that people have been transient for a long time. Whereas last year, people were more inclined to get their choice and then stay in the area which they were [in] to develop that area. I think this year they have been given the same option to develop in their area and if they want to shift then they have been allowed to move around, which I think, just makes everybody feel more stable and able to get a handle on something; instead of just getting in there, just getting used to something and doing it and then off you go. I suppose I've kept on, even though I haven't been Computer Coordinator this year, I've worked closely with Eric and learned a lot from Eric because he's so very knowledgeable anyway. But he's great to work with and I said to him last year... what I felt I needed was somebody else to work with me (being new and not knowing anything, and having, 'Here it's all yours, your computer you must do all this'). Even though at the time it made me learn a lot, I felt in that area you definitely need some support. So, I stayed with him to give him support this year. I think, with people staying in some of the KLA areas (most of those remained the same this year which was good because people, instead of learning all new things every year, have actually had a chance to develop what they've learned). I think that's a big factor. 


\section{Appendix 5: Analysis of an interview with a leading teacher (Interview B8)}

Apart from the name of the interviewer (the author of this thesis), all the names provided in the following extract are pseudonyms.

Andrew (15a): Now can you please describe how this team [the Five-Six Area Team] operates and what you've achieved together recently?

Margaret (15a): This year has been turmoil plus! Only because the man I was working with, Barry, went on sick leave partly through last term (right at the beginning of the last term). Consequently, I had to work with a number of different people in the room, trying to keep the grade settled, so it was very important to work with them. Now that there is another person in the room, Eric, I have to work closely with Eric to make sure he can see what's going on and letting him know what has happened, where we are going from here. [There is] lots of planning that we have to do, from excursions and getting ready for our camp next year down to what's been taught each week in the room. Working through that and how to assess it and all the assessment that is coming up. Making sure that he is aware of what school policies are on all the different things and working through that.

Andrew (15b): It must be pretty difficult getting together to talk when you've got such a busy classroom schedule?

Margaret (15b): We'll plan times for meetings (which are Wednesday night after school) but otherwise we would talk every day. I make sure (especially because he is new) I go and see him every day. After school [we] talk about things, whenever we can anyway, or during a recess or a lunch.

Andrew (15c): I know how hard it is to come into a school for the first time; it's very difficult. 
Margaret (15c): We've done all our major planning for this term together which was good, so that he has a very good idea of what to do. It makes him feel a lot better. Also, I'm working with him on the computer literacy because he hasn't used computers before. We're trying to do a lot of our planning on the computer with him, so he can see [how the computer can be used]. I've got him now taking the laptop home and using the laptop; he learned how to use it last week, it was good.

\section{Analysis}

Here we have an account of various connections, between people, environment (the classroom) and technological artefacts (computers). The problem, as described by Margaret, relates to the organisational functioning of the school. It was dealt with in a dynamic manner, so that as the equilibrium of the situation is disturbed by the departure of Barry, Margaret works with various other people to restore and maintain the needed balance. Here, the learning of one organisational member occurs as he is introduced in situ to various culturally relevant organisational matters (excursion and camp planning, school policies, and assessment schedules). This account sits comfortably within distributed cognition theory.

Another account may be given, by which Margaret's actions are construed as leadership. Such an account will rely on the perceived recognition of certain leadership schemas with their related leadership qualities. This account could be interpreted according to any one, or two, or all of the schemas of leadership-ascompetence, leadership-as-influence, or leadership-as-social-relations. The extent to which any one schema is enacted will depend upon the perspective of the observer as he or she fills in the missing information in the narrative and interprets the foreground and background features of the resulting scene or image.

Hence, two accounts may arise from the one information space (as provided by the narrative in this case). One account analyses the causal computational interactions of culture, artefacts and environment, and the emotional responses of the people involved. This analysis is done from a detached, objective standpoint - from the outside-looking-in. 
The other account places the reader within the narrative, feeling what it would be like to be Eric - feeling inspired by the information provided, or more at ease with an unfamiliar situation, or feeling satisfied with a new found familiarity with technology. Alternatively, the reader might imagine what it would be like to be in Margaret's position, recognising what needs to be done, building relations of trust and acceptance with others, sharing knowledge, and feeling satisfied by what eventuates. This is an analysis conducted from a subjective, personal standpoint from the inside-looking-out.

Whilst this extract may be construed as leadership, its theoretical value is in the causal-computational interactions of the situation. However, there is also value in an individual construing the account in a phenomenological way so that insight is obtained into what it means to experience what the participants experience. This is a very difficult thing to prescribe or generalise - it is a very personal matter and may have very different outcomes for different people. 


\section{University Library}

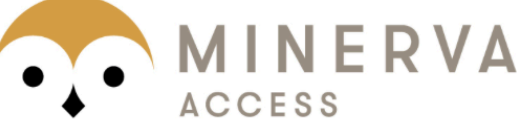

A gateway to Melbourne's research publications

Minerva Access is the Institutional Repository of The University of Melbourne

Author/s:

Hill, Andrew Leonard

Title:

Distributed leadership in schools

Date:

2008

Citation:

Hill, A. L. (2008). Distributed leadership in schools. PhD thesis, Faculty of Education, Education, University of Melbourne.

Publication Status:

Unpublished

Persistent Link:

http://hdl.handle.net/11343/39489

Terms and Conditions:

Terms and Conditions: Copyright in works deposited in Minerva Access is retained by the copyright owner. The work may not be altered without permission from the copyright owner. Readers may only download, print and save electronic copies of whole works for their own personal non-commercial use. Any use that exceeds these limits requires permission from the copyright owner. Attribution is essential when quoting or paraphrasing from these works. 